\title{
Allylic Amination of Alkenes with Iminothianthrenes to Afford Alkyl Allyl Amines
}

Qiang Cheng, Junting Chen, Songyun Lin, and Tobias Ritter*

\author{
Max-Planck-Institute für Kohlenforschung, \\ Kaiser-Wilhelm Platz 1, D-45470 Mülheim an der Ruhr \\ *E-mail: ritter@kofo.mpg.de
}




\section{TABLE OF CONTENTS}

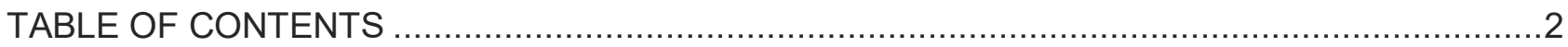

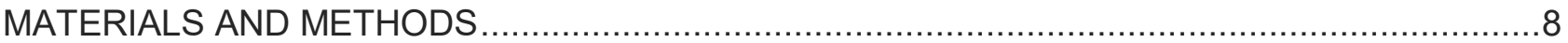

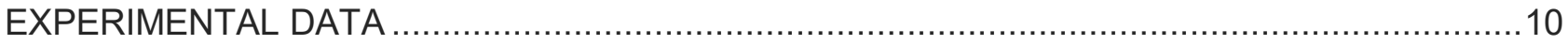

General procedure and reaction condition optimization for allylic C-H amination ................................ 10

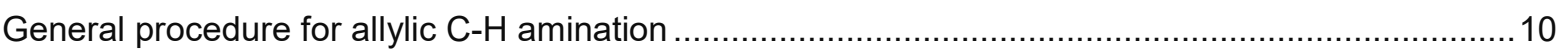

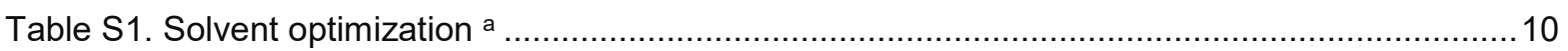

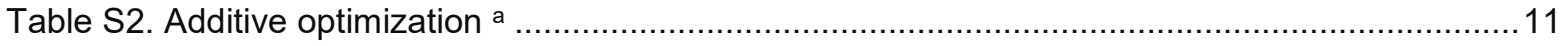

Table S3. Light source and temperature optimization a …......................................................... 11

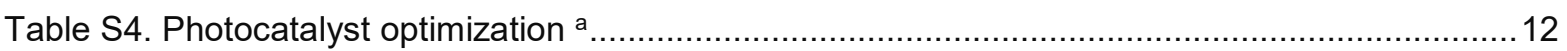

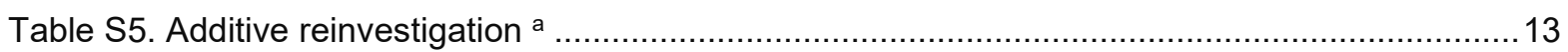

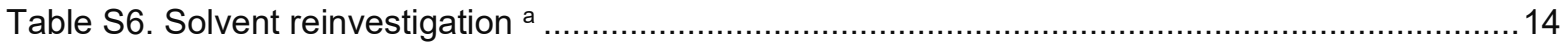

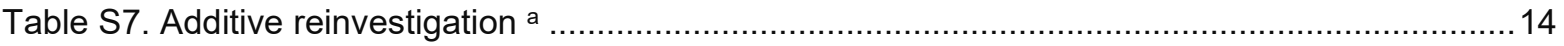

Table S8. Investigation of byproduct in different temperature a .................................................. 15

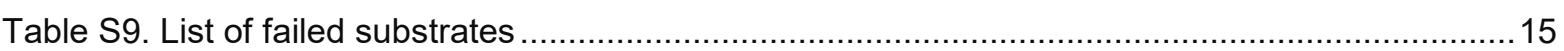

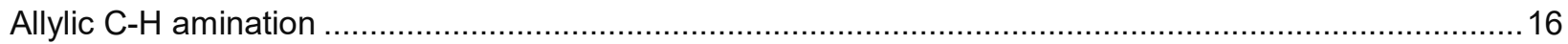

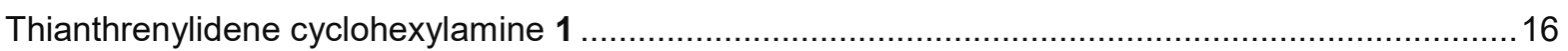

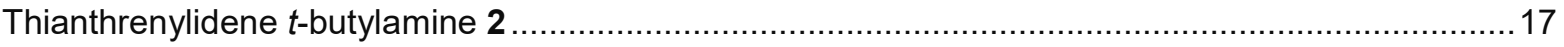

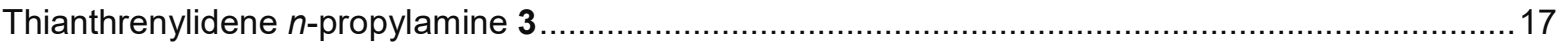

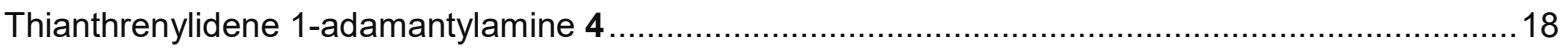

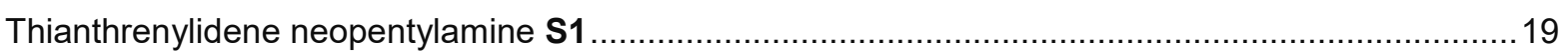

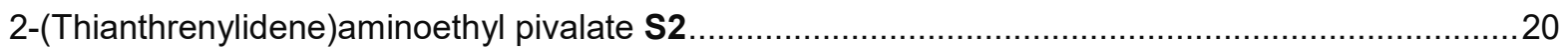

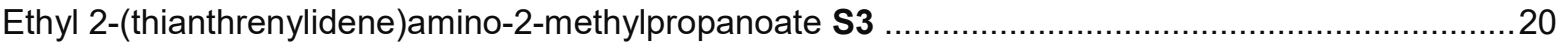

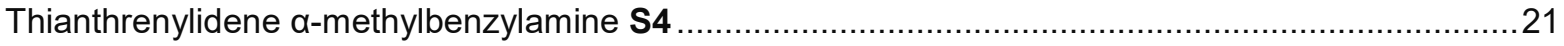

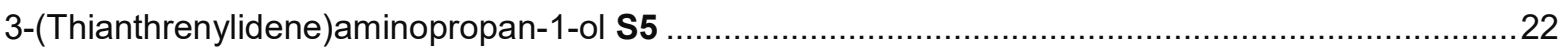

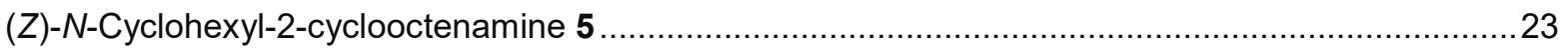

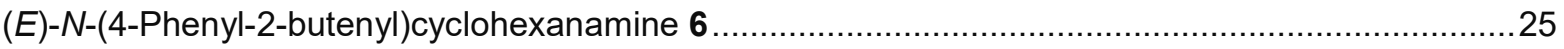

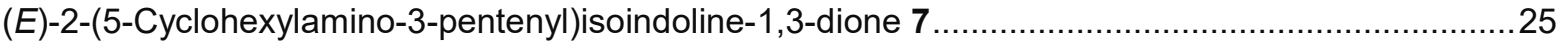

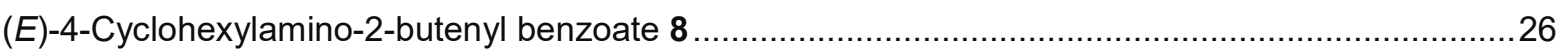

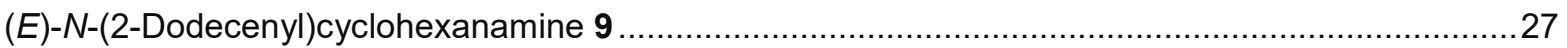

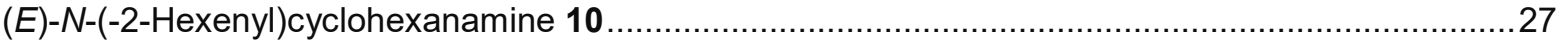

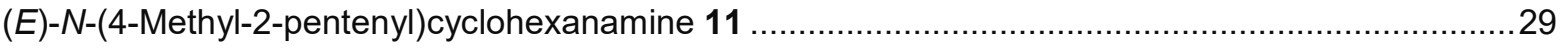

(E)-N-(5-(tert-Butyldiphenylsilyl)oxy-2-pentenyl)cyclohexanamine 12 ........................................29

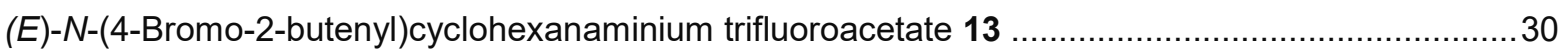

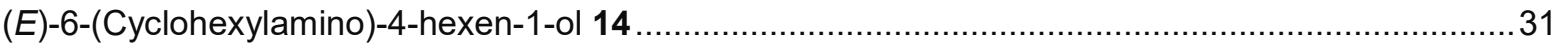

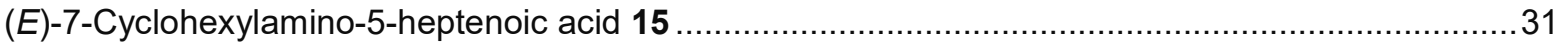




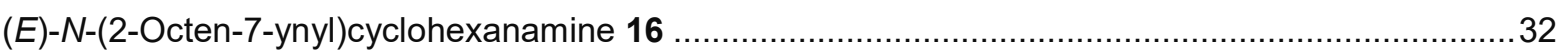

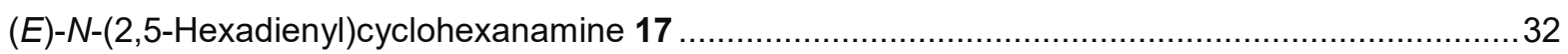

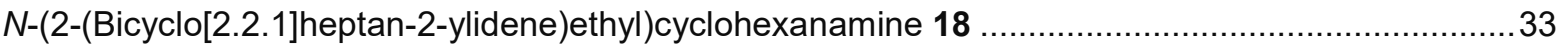

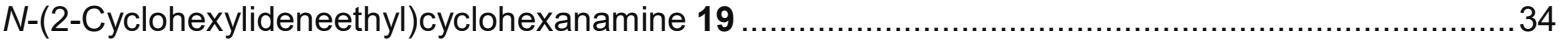

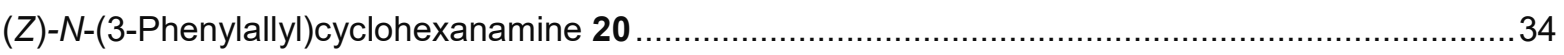

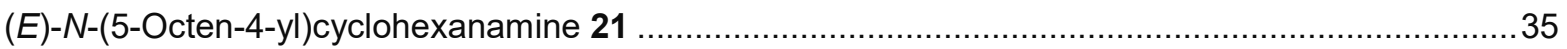

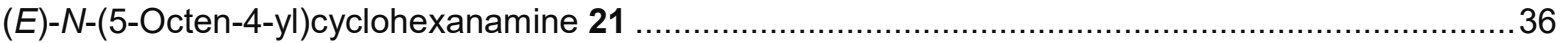

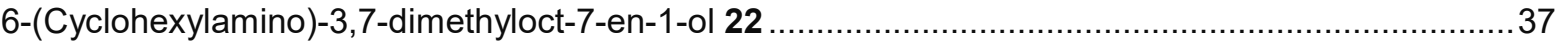

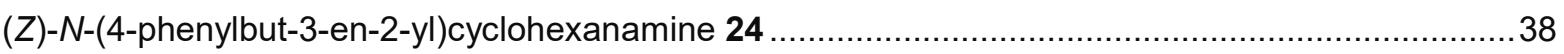

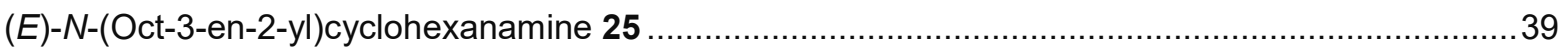

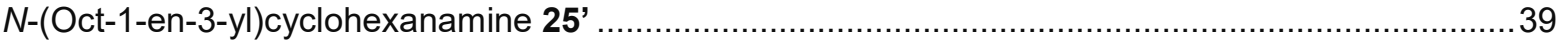

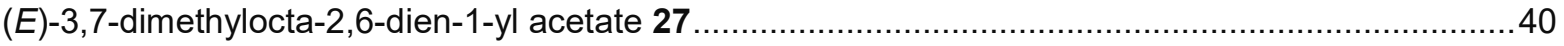

(E)-6-(Cyclohexylamino)-3,7-dimethylocta-2,7-dien-1-yl acetate 26 .......................................... 41

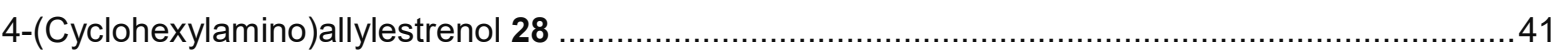

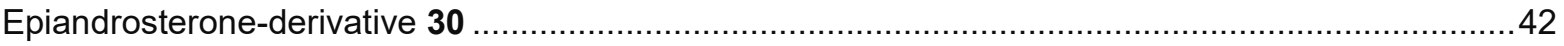

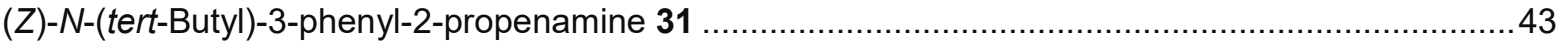

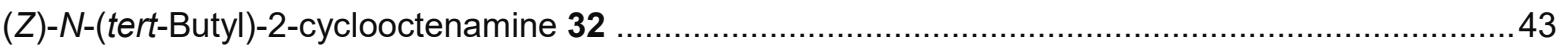

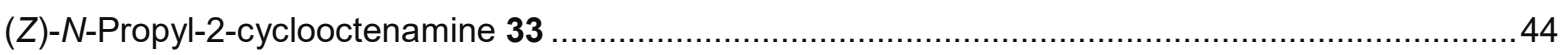

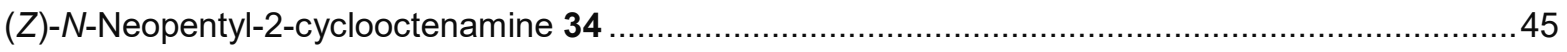

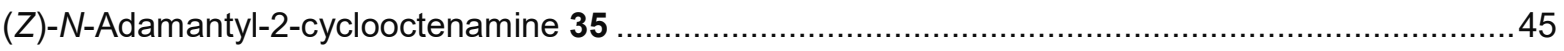

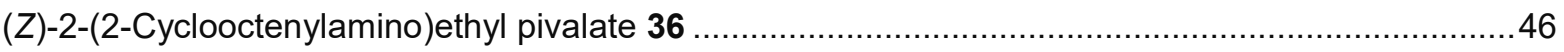

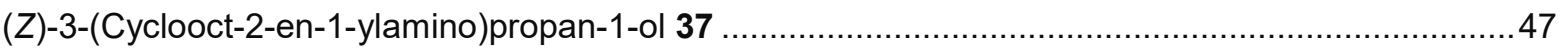

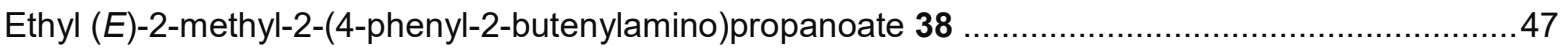

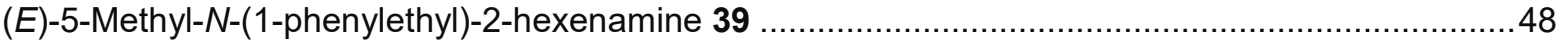

$N$-(tert-Butyl)-3-phenyl-2-((2,2,6,6-tetramethylpiperidin-1-yl)oxy)propanamine 40

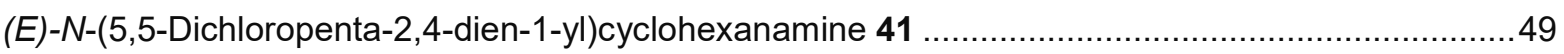

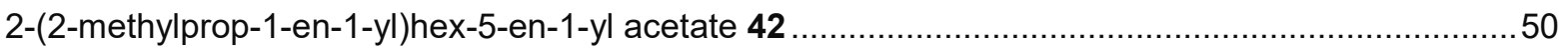

(3-((Cyclohexylamino)methyl)-2-(prop-1-en-2-yl)cyclopentyl)methyl acetate 43 ….........................51

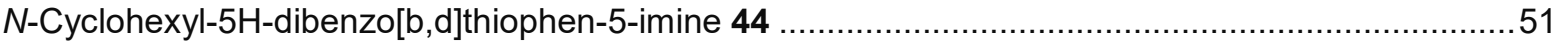

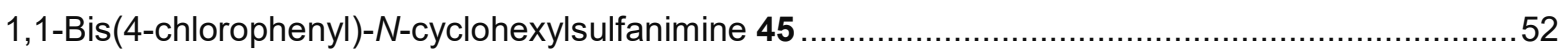

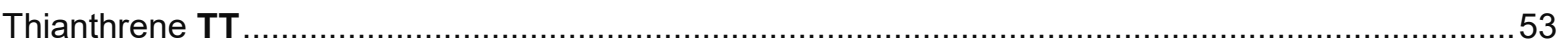

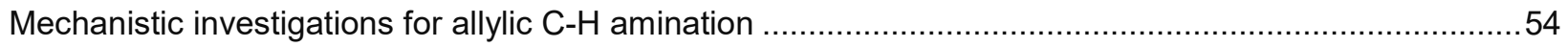

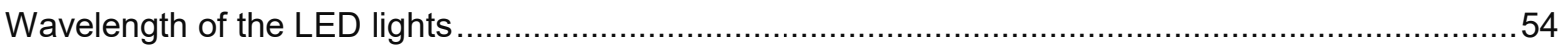

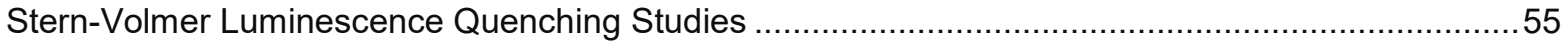

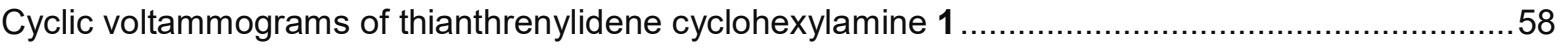

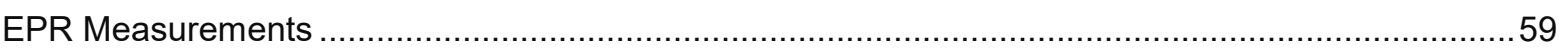

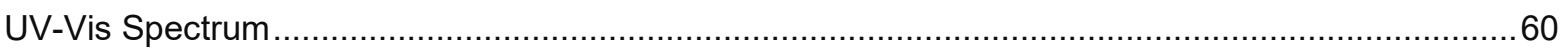

Proposed mechanism for the formation of regioisomers when using cyclooctene as substrate .........61 
Procedure for isomerization of $(E)$-cinnamylamine $\mathbf{4 6}$ under photocatalysis.

Procedure for reaction under UV light.

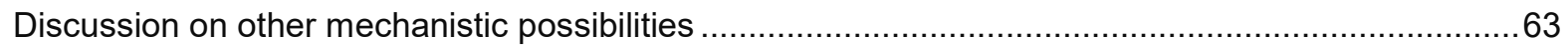

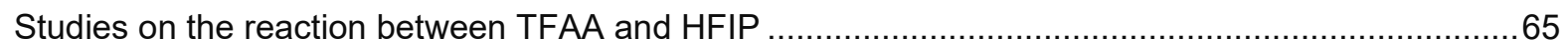

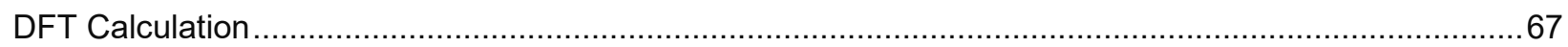

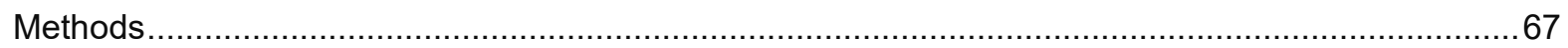

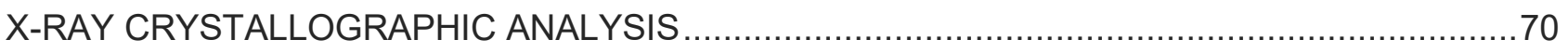

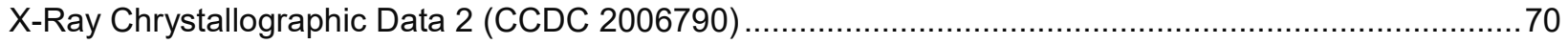

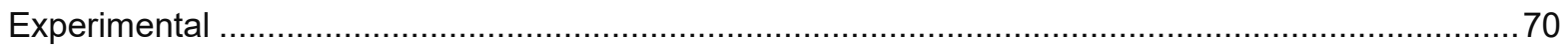

Table S10. Crystal data and structure refinement. ................................................................. 70

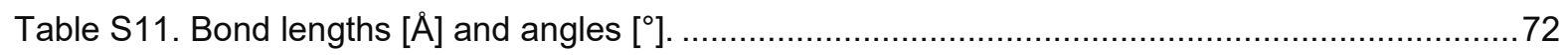

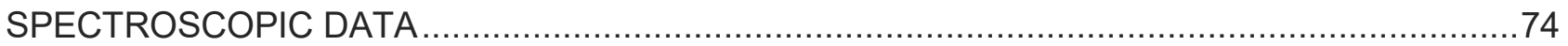

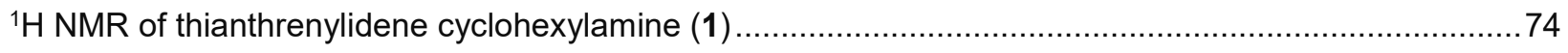

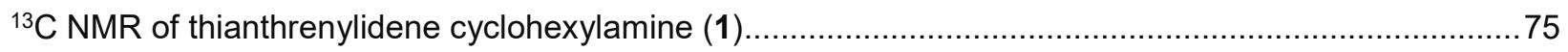

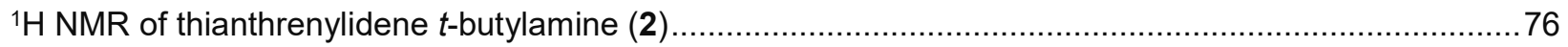

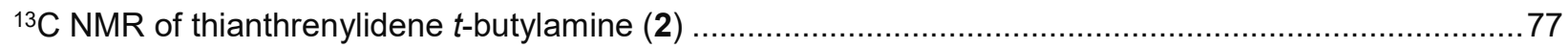

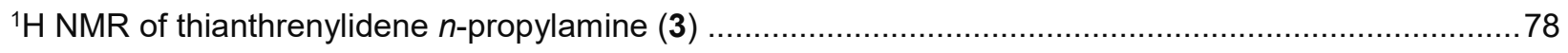

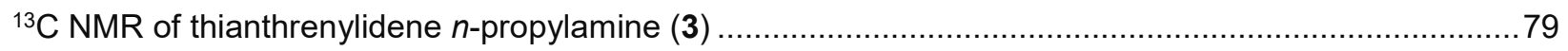

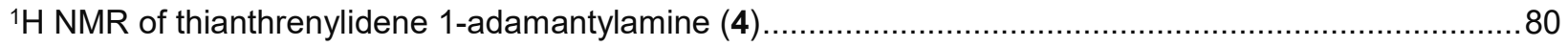

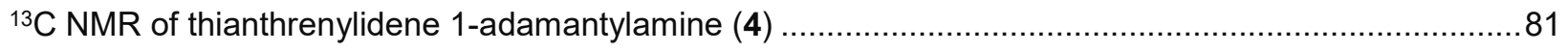

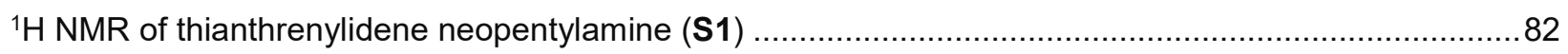

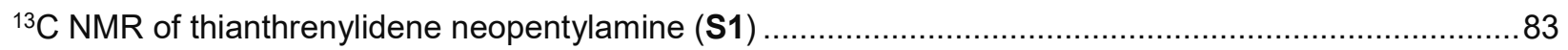

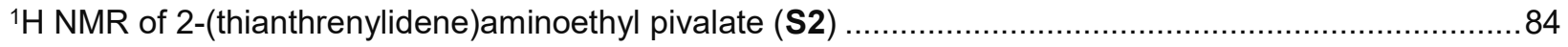

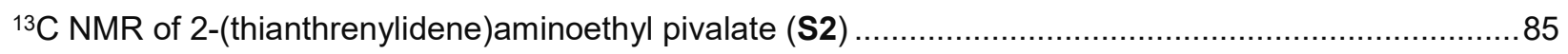

${ }^{1} \mathrm{H}$ NMR of ethyl 2-(thianthrenylidene)amino-2-methylpropanoate (S3) ............................................ 86

${ }^{13} \mathrm{C}$ NMR of ethyl 2-(thianthrenylidene)amino-2-methylpropanoate (S3) …...................................... 87

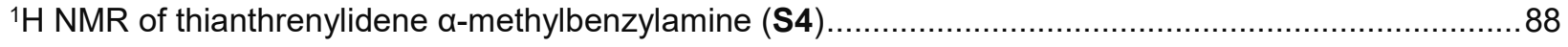

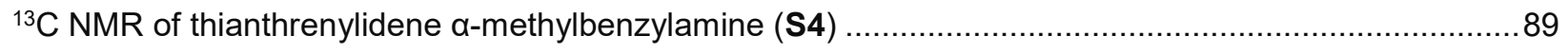

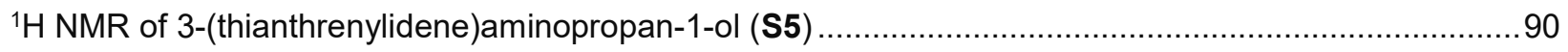

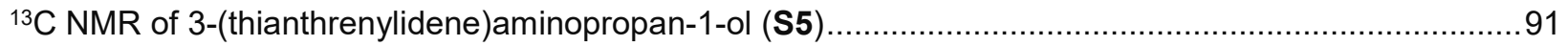


${ }^{1} \mathrm{H}$ NMR of (Z)-N-Cyclohexyl-2-cyclooctenamine (5)

${ }^{13} \mathrm{C}$ NMR of (Z)-N-Cyclohexyl-2-cyclooctenamine (5)

${ }^{1} \mathrm{H}$ NMR of $(E)-\mathrm{N}$-(4-phenyl-2-butenyl)cyclohexanamine (6). .94

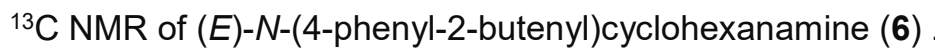
.95

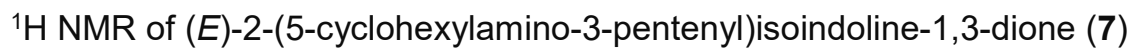
.96

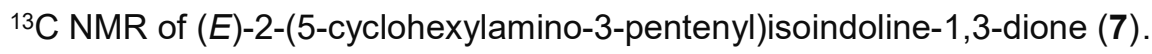
.97

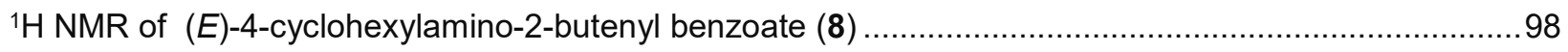

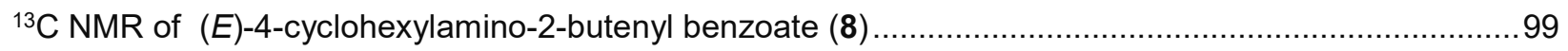

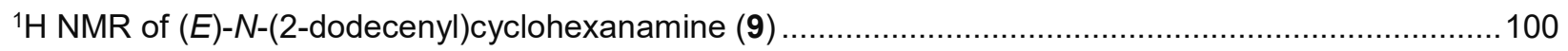

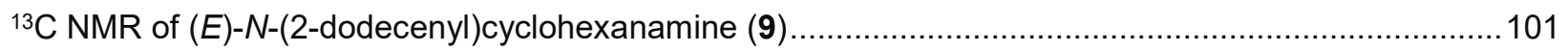

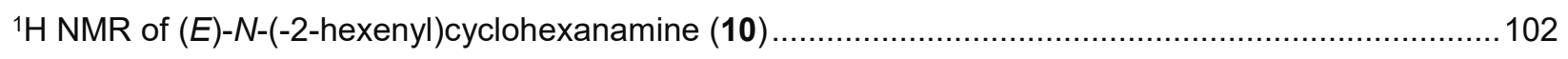

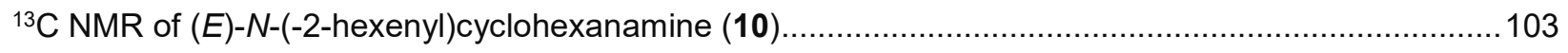

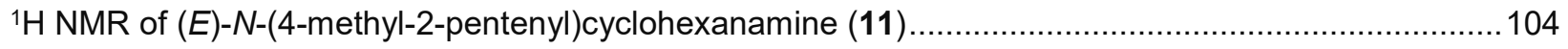

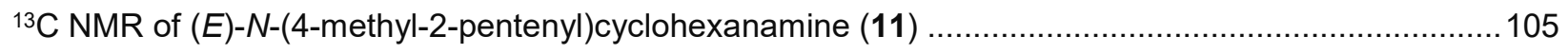

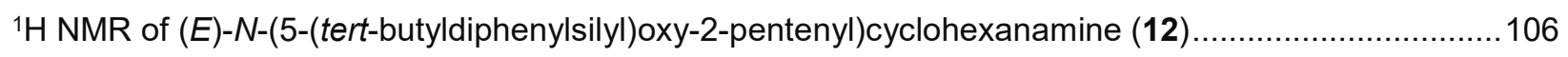

${ }^{13} \mathrm{C}$ NMR of $(E)-\mathrm{N}$-(5-(tert-butyldiphenylsilyl)oxy-2-pentenyl)cyclohexanamine (12) ............................ 107

${ }^{1} \mathrm{H}$ NMR of $(E)-\mathrm{N}-(4-$ bromo-2-butenyl)cyclohexanaminium trifluoroacetate (13) .................................. 108

${ }^{13} \mathrm{C}$ NMR of $(E)-\mathrm{N}$-(4-bromo-2-butenyl)cyclohexanaminium trifluoroacetate (13) ................................109

${ }^{19} \mathrm{~F}$ NMR of $(E)-\mathrm{N}$-(4-bromo-2-butenyl)cyclohexanaminium trifluoroacetate (13) ................................110

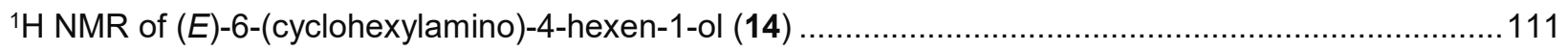

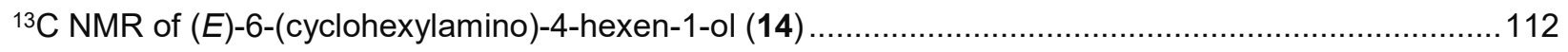

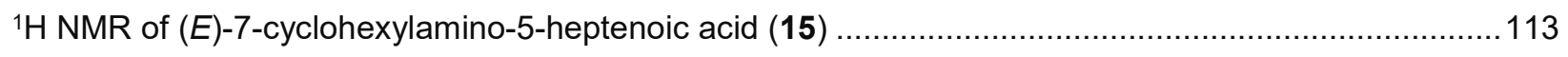

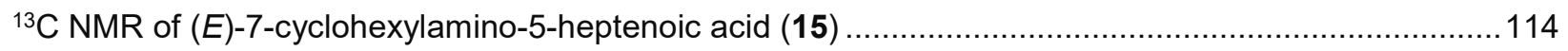

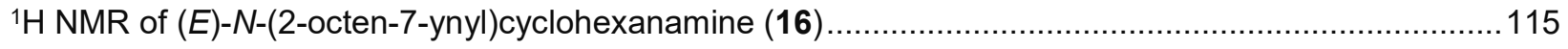

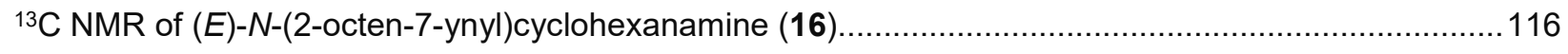

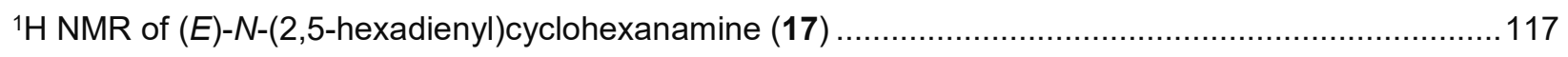

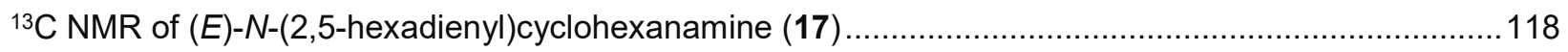

${ }^{1} \mathrm{H}$ NMR of $\mathrm{N}$-(2-(bicyclo[2.2.1] heptan-2-ylidene)ethyl)cyclohexanamine (18) ...................................119

${ }^{13} \mathrm{C}$ NMR of $\mathrm{N}$-(2-(bicyclo[2.2.1]heptan-2-ylidene)ethyl)cyclohexanamine (18) ..................................120 
${ }^{1} \mathrm{H}$ NMR of $\mathrm{N}$-(2-cyclohexylideneethyl)cyclohexanamine (19)

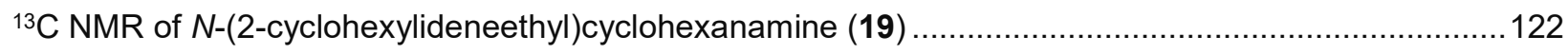

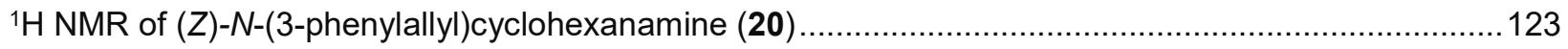

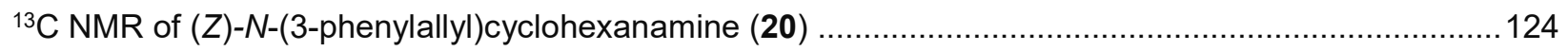

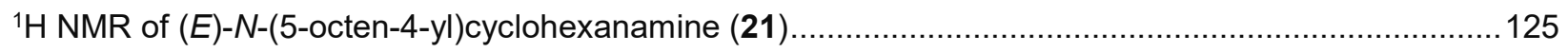

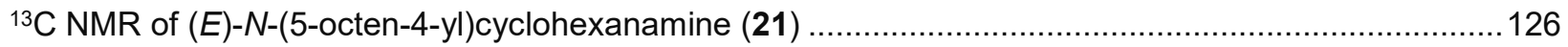

${ }^{1}$ H NMR of 6-(cyclohexylamino)-3,7-dimethyloct-7-en-1-ol (22) ................................................... 127

${ }^{13} \mathrm{C}$ NMR of 6-(cyclohexylamino)-3,7-dimethyloct-7-en-1-ol (22) ..................................................128

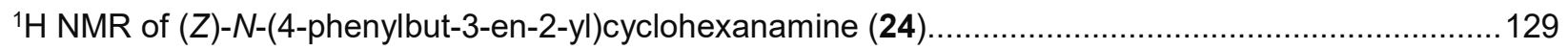

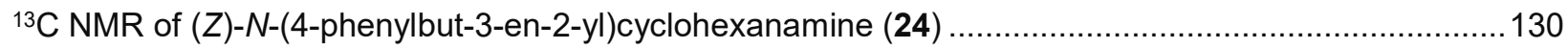

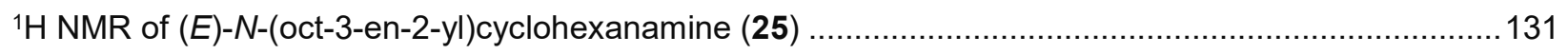

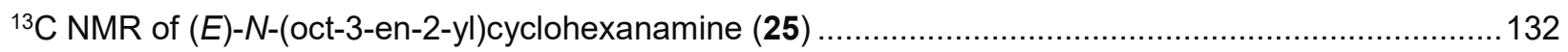

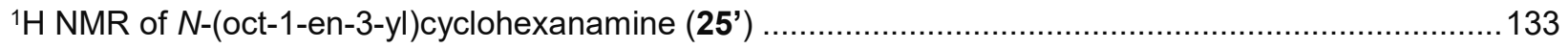

${ }^{1} \mathrm{H}$ NMR of (E)-6-(cyclohexylamino)-3,7-dimethylocta-2,7-dien-1-yl acetate (26)............................... 134

${ }^{13} \mathrm{C}$ NMR of (E)-6-(cyclohexylamino)-3,7-dimethylocta-2,7-dien-1-yl acetate (26) .............................. 135

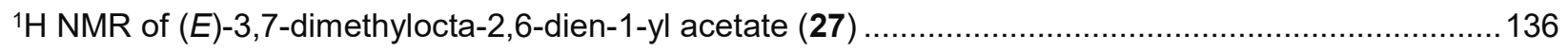

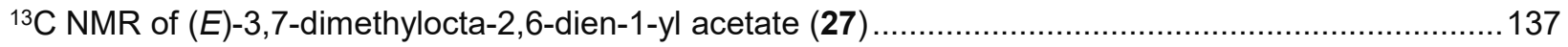

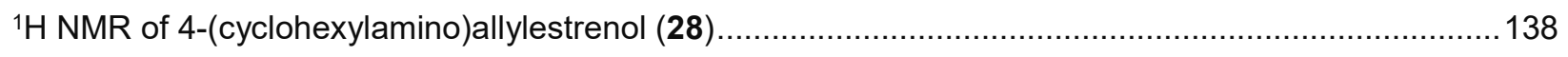

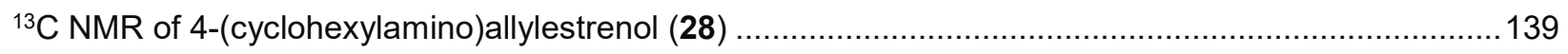

${ }^{1} \mathrm{H}-{ }^{1} \mathrm{H}$ COSY spectrum of 4-(cyclohexylamino)allylestrenol (28)................................................... 140

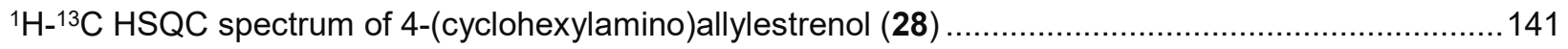

${ }^{1} \mathrm{H}-{ }^{13} \mathrm{C}$ HMBC spectrum of 4-(cyclohexylamino)allylestrenol (28) ................................................. 142

${ }^{1} \mathrm{H}-{ }^{1} \mathrm{H}$ NOESY spectrum of 4-(cyclohexylamino)allylestrenol (28) .................................................. 143

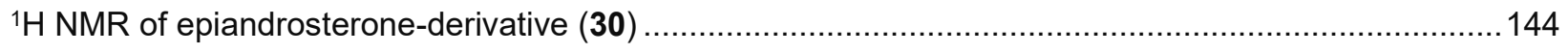

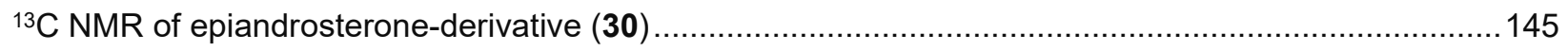

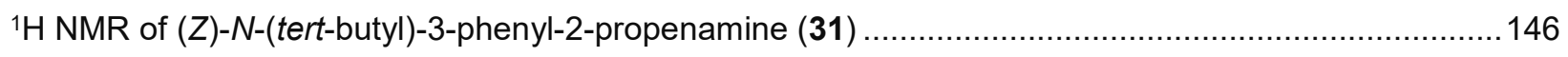

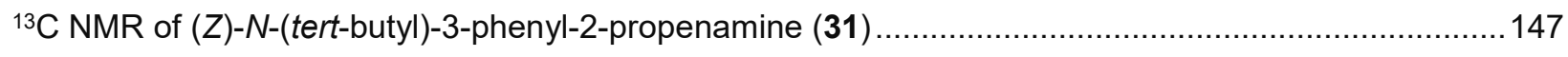

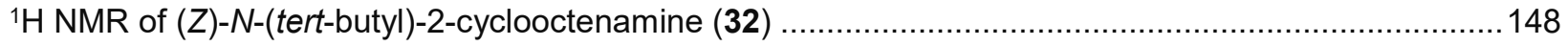

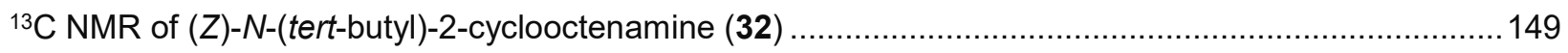


${ }^{1} \mathrm{H}$ NMR of (Z)-N-propyl-2-cyclooctenamine (33) 150

${ }^{13} \mathrm{C}$ NMR of (Z)- $N$-propyl-2-cyclooctenamine (33) 151

${ }^{1} \mathrm{H}$ NMR of (Z)-N-neopentyl-2-cyclooctenamine (34) 152

${ }^{13} \mathrm{C}$ NMR of (Z)-N-neopentyl-2-cyclooctenamine (34) 153

${ }^{1} \mathrm{H}$ NMR of (Z)-N-adamantyl-2-cyclooctenamine (35) 154

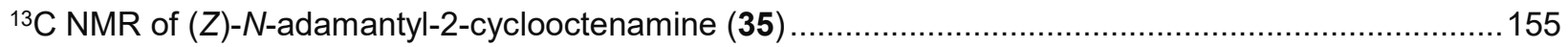

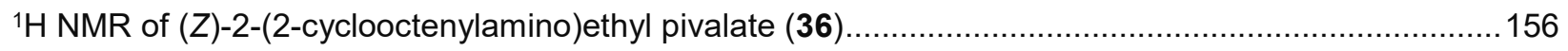

${ }^{13} \mathrm{C}$ NMR of (Z)-2-(2-cyclooctenylamino)ethyl pivalate (36) ...................................................... 157

${ }^{1} \mathrm{H}$ NMR of (Z)-3-(cyclooct-2-en-1-ylamino)propan-1-ol (37).................................................... 158

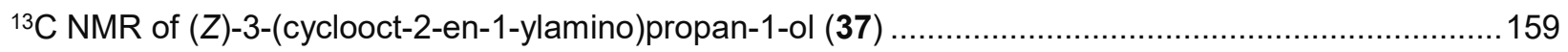

${ }^{1} \mathrm{H}$ NMR of ethyl (E)-2-methyl-2-(4-phenyl-2-butenylamino)propanoate (38) ..................................... 160

${ }^{13} \mathrm{C}$ NMR of ethyl (E)-2-methyl-2-(4-phenyl-2-butenylamino)propanoate (38) .....................................161

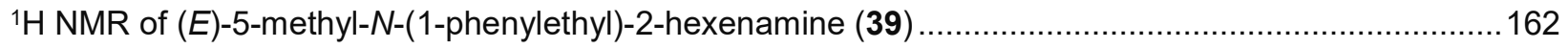

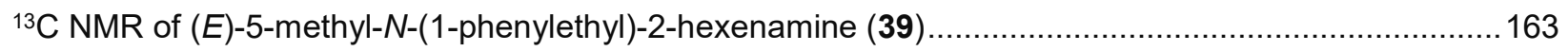

${ }^{1} \mathrm{H}$ NMR of $\mathrm{N}$-(tert-butyl)-3-phenyl-2-((2,2,6,6-tetramethylpiperidin-1-yl)oxy)propanamine (40)..............164

${ }^{13} \mathrm{C}$ NMR of $\mathrm{N}$-(tert-butyl)-3-phenyl-2-((2,2,6,6-tetramethylpiperidin-1-yl)oxy)propanamine (40) .............165

${ }^{1} \mathrm{H}$ NMR of $(E)-\mathrm{N}$-(5,5-dichloropenta-2,4-dien-1-yl)cyclohexanamine (41) ......................................... 166

${ }^{13} \mathrm{C}$ NMR of $(E)-\mathrm{N}$-(5,5-dichloropenta-2,4-dien-1-yl)cyclohexanamine (41) .......................................167

${ }^{1}$ H NMR of 2-(2-methylprop-1-en-1-yl)hex-5-en-1-yl acetate (42)................................................. 168

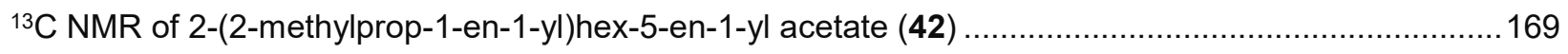

${ }^{1} \mathrm{H}$ NMR of (3-((cyclohexylamino)methyl)-2-(prop-1-en-2-yl)cyclopentyl)methyl acetate (43) .................170

${ }^{13} \mathrm{C}$ NMR of (3-((cyclohexylamino)methyl)-2-(prop-1-en-2-yl)cyclopentyl)methyl acetate (43) ................171

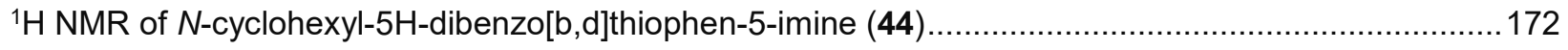

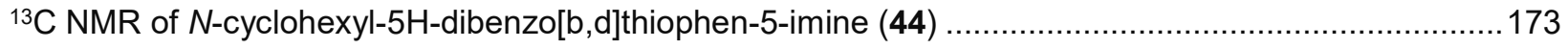

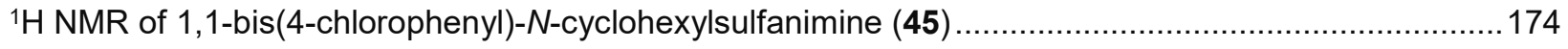

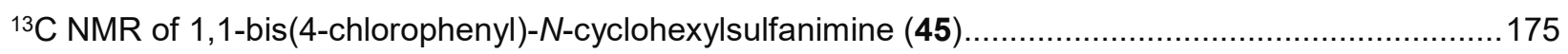

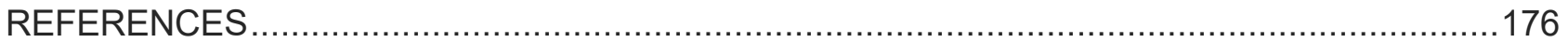




\section{MATERIALS AND METHODS}

All reactions were carried out under ambient atmosphere unless otherwise stated and monitored by thin-layer chromatography (TLC). High-resolution mass spectra were obtained using $Q$ Exactive Plus from Thermo. Concentration under reduced pressure was performed by rotary evaporation at $25-40{ }^{\circ} \mathrm{C}$ at an appropriate pressure. Purified compounds were further dried under vacuum $\left(10^{-6}-10^{-3}\right.$ bar $)$. Yields refer to purified and spectroscopically pure compounds, unless otherwise stated.

\section{Solvents}

HFIP was purchased from Oakwood Chemicals and dried with $3 \AA$ molecular sieves. Anhydrous solvents were obtained from Phoenix Solvent Drying Systems. All deuterated solvents were purchased from Euriso-Top .

\section{Chromatography}

Thin layer chromatography (TLC) was performed using EMD TLC plates pre-coated with $250 \mu \mathrm{m}$ thickness silica gel $60 \mathrm{~F}_{254}$ plates and visualized by fluorescence quenching under UV light and $\mathrm{KMnO}_{4}$ stain. Flash column chromatography was performed using silica gel (40-63 $\mu \mathrm{m}$ particle size) purchased from Geduran®.

\section{Photochemistry}

All reactions with blue light were carried out using a photoreactor equipped with a blue LED module (KTElektronik, "100W Power LED blau 450 nm Aquarium”, 450 nm, 100 W), consisting out of 100 LED-chips. The power of the LED was adjusted using a linear regulator. The vials were cooled with two Peltier-elements (TEC1-12706) while being irradiated with blue light. All reactions with purple light were carried out using a photoreactor equipped with a purple LED module $(400 \mathrm{~nm}, 200 \mathrm{~W})$. The power of the LED was adjusted using a linear regulator. The vials were cooled with two Peltier-elements (TEC1-12706) while being irradiated with purple light.

\section{Spectroscopy and Instruments}

NMR spectra were recorded on a Bruker Ascend ${ }^{T M} 500$ spectrometer operating at $500 \mathrm{MHz}, 471 \mathrm{MHz}$, and $126 \mathrm{MHz}$, for ${ }^{1} \mathrm{H},{ }^{19} \mathrm{~F}$, and ${ }^{13} \mathrm{C}$ acquisitions, respectively; or on a Varian Unity/Inova 600 spectrometer operating at $600 \mathrm{MHz}$ and $151 \mathrm{MHz}$ for ${ }^{1} \mathrm{H}$ and ${ }^{13} \mathrm{C}$ acquisitions, respectively. Chemical shifts are reported in ppm with the solvent residual peak as the internal standard. For ${ }^{1} \mathrm{H}$ NMR: $\mathrm{CDCl}_{3}, \delta 7.26 ; \mathrm{CD}_{3} \mathrm{OD}, \delta 3.31$. For ${ }^{13} \mathrm{C}$ NMR: $\mathrm{CDCl}_{3}, \delta 77.16 ; \mathrm{CD}_{3} \mathrm{OD}, \delta 49.00 .{ }^{1}{ }^{19} \mathrm{~F}$ NMR spectra were referenced using a unified chemical shift scale based on the ${ }^{1} \mathrm{H}$ resonance of tetramethylsilane $(1 \%(\mathrm{v} / \mathrm{v})$ solution in the respective solvent). Data is reported as follows: $\mathrm{s}=$ singlet, $\mathrm{d}=$ doublet, $\mathrm{t}=$ triplet, $\mathrm{q}=$ quartet, $\mathrm{m}=$ multiplet, $\mathrm{br}=$ broad; coupling constants in $\mathrm{Hz}$; integration.

\section{Starting materials}

All substrates were used as received from commercial suppliers, unless otherwise stated. Alkenes were purchased from Sigma-Aldrich, Chempur, TCl, or Alfa Aesar. Thianthrene-S-oxide (TTO) was prepared 
according to the literature. ${ }^{2}$ 


\section{EXPERIMENTAL DATA}

\section{General procedure and reaction condition optimization for allylic $\mathrm{C}-\mathrm{H}$ amination}

General procedure for allylic C-H amination

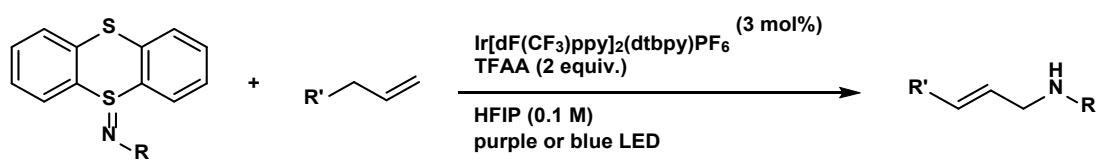

Under nitrogen atmosphere, to a 4-mL borosilicate vial equipped with a magnetic stir bar were added alkyl thianthrenylidene amine ( $0.100 \mathrm{mmol}, 1.00$ equiv. $)$, alkene $(0.150 \mathrm{mmol}, 1.50$ equiv. $)$,

$\operatorname{Ir}\left[\mathrm{dF}\left(\mathrm{CF}_{3}\right) \mathrm{ppy}_{2}(\mathrm{dtbpy}) \mathrm{PF}_{6}(3.3 \mathrm{mg}, 3.0 \mu \mathrm{mol}, 3.0 \mathrm{~mol} \%), \mathrm{HFIP}(1 \mathrm{~mL}, \mathrm{c}=0.1 \mathrm{M})\right.$, and trifluoroacetic anhydride $\left(28 \mu \mathrm{L}, 42 \mathrm{mg}, 0.20 \mathrm{mmol}, 2.0\right.$ equiv.). The vial was sealed with a septum-cap and irradiated for $3 \mathrm{~h}$ at $5{ }^{\circ} \mathrm{C}$ using a purple LED $(30 \mathrm{~W})$ or at $8{ }^{\circ} \mathrm{C}$ using a blue LED $(30 \mathrm{~W})$. Then, the reaction mixture was concentrated. The residue was dissolved in DCM $(5 \mathrm{~mL})$ and washed with saturated sodium carbonate aqueous solution (5 $\mathrm{mL})$. The aqueous phase was extracted with $\mathrm{DCM}(2 \times 5 \mathrm{~mL})$. The organic phase was dried over $\mathrm{Na}_{2} \mathrm{SO}_{4}$ and the solvent was removed under reduced pressure. The residue was purified by chromatography on silica gel eluting with $\mathrm{CHCl}_{3} / \mathrm{MeOH}(50 / 1-30 / 1(\mathrm{v} / \mathrm{v}))$ to afford the amination product. The $\mathrm{E} / \mathrm{Z}$ isomers were not separated and were collected together. The ratio was determined by appropriate integration of the peaks of allylic protons at 3 to $4 \mathrm{ppm}$ in the ${ }^{1} \mathrm{H}$ NMR spectra.

Note: The reaction is air sensitive. Schlenk techniques were used to avoid air. For simplicity, in our own research, we have opted to execute the transformation for most compounds by using a glovebox. Control experiments showed that yields were within error of measurement if the reaction was carried out using a glovebox or not.

Table S1. Solvent optimization ${ }^{\text {a }}$

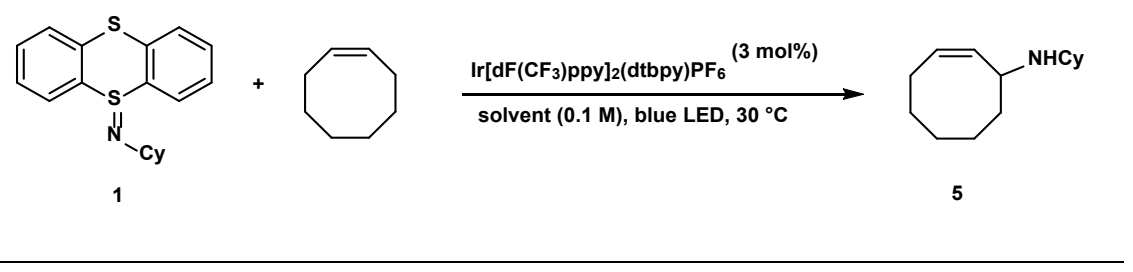

\begin{tabular}{ll} 
solvent & yield $(\%)^{b}$ \\
\hline MeCN & 0 \\
THF & 0 \\
DCM & 0
\end{tabular}


a 1 (0.05 mmol), cyclooctene ( $0.075 \mathrm{mmol}, 1.5$ equiv.), $\operatorname{Ir}\left[\mathrm{dF}\left(\mathrm{CF}_{3}\right) \text { ppy }\right]_{2}(\mathrm{dtbpy}) \mathrm{PF} 6$ (3 mol\%), solvent (0.1 M), blue LED $(80 \mathrm{~W}), 30{ }^{\circ} \mathrm{C} .{ }^{b} \mathrm{NMR}$ yield with $\mathrm{CH}_{2} \mathrm{Br}_{2}$ as internal standard.

Table S2. Additive optimization ${ }^{a}$

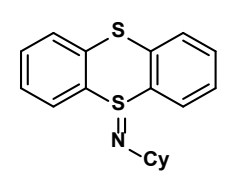

1

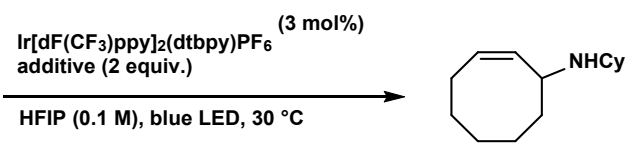

5

\begin{tabular}{ll}
\hline additive & yield $(\%)^{b}$ \\
\hline none & 20 \\
TFAA & 55 \\
TFA & 28 \\
$\mathrm{Ac}_{2} \mathrm{O}$ & 25 \\
$\mathrm{Tf}_{2} \mathrm{O}$ & 16 \\
$\mathrm{HClO}_{4}$ & 18 \\
\hline
\end{tabular}

a 1 (0.05 mmol), cyclooctene ( $0.075 \mathrm{mmol}, 1.5$ equiv.), $\operatorname{Ir}\left[\mathrm{dF}\left(\mathrm{CF}_{3}\right) \text { ppy }\right]_{2}(\mathrm{dtbpy}) \mathrm{PF}_{6}$ (3 mol\%), additive (2 equiv.), HFIP (0.1 M), blue LED (80 W), $30{ }^{\circ} \mathrm{C}$. ${ }^{b} \mathrm{NMR}$ yield with $\mathrm{CH}_{2} \mathrm{Br}_{2}$ as internal standard.

Table S3. Light source and temperature optimization a

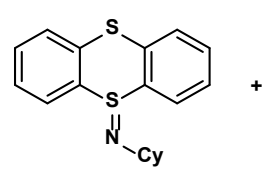

1

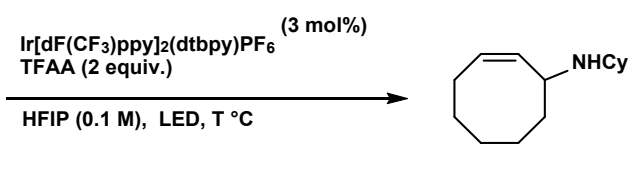

5

light source $\quad$ temperature $\left({ }^{\circ} \mathrm{C}\right) \quad$ yield $(\%)^{\mathrm{b}}$

none

25 
Blue LED (80 W)

Blue LED (60 W)

Blue LED (30 W)

Blue LED (30 W)

Purple LED (30 W)

Purple LED $(30 \mathrm{~W})^{\mathrm{e}}$

Purple LED $(30 \mathrm{~W})^{\mathrm{f}}$
30

20

8

8

5

5

5
55

64

75

$45^{c}$

$80(77)^{d}$

60

42

a 1 (0.05 mmol), cyclooctene ( 0.075 mmol, 1.5 equiv.), Ir[dF( $\left.\mathrm{CF}_{3}\right)$ ppy $]_{2}(\mathrm{dtbpy}) \mathrm{PF}_{6}$ (3 mol\%), TFAA (2 equiv.), HFIP $(0.1 \mathrm{M})$, light. ${ }^{b} \mathrm{NMR}$ yield with $\mathrm{CH}_{2} \mathrm{Br}_{2}$ as internal standard. ${ }^{c}$ without TFAA. ${ }^{d}$ Isolated yield in the parentheses. e 1 :cyclooctene $=1: 1 .{ }^{\mathrm{f}} \mathbf{1}$ : cyclooctene $=1.5: 1$.

Table S4. Photocatalyst optimization ${ }^{a}$

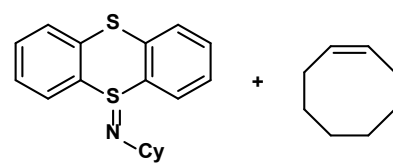

1

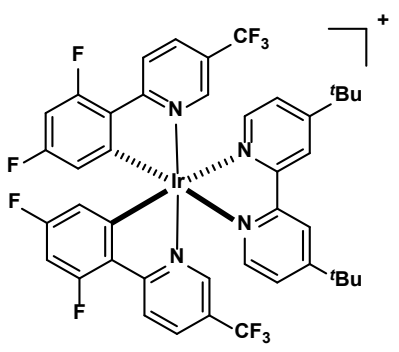

$\operatorname{Ir}\left[\mathrm{dF}\left(\mathrm{CF}_{3}\right) \text { ppy }\right]_{2}$ (dtbpy)PF 6 $E\left(\operatorname{Ir}(I I I)^{\star} / \operatorname{lr}(I I)\right)=1.21 \mathrm{~V}$ $E(\operatorname{Ir}(I I I) * / / r(I V))=-0.89 \mathrm{~V}$

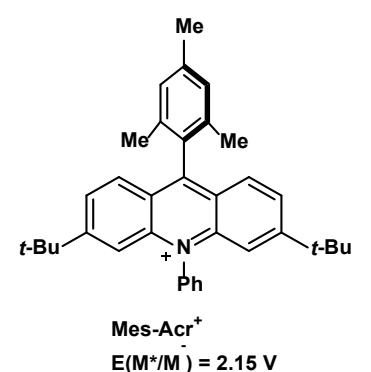

photocatalyst (3 mol\%)

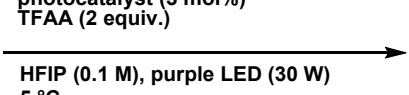

$5{ }^{\circ} \mathrm{C}$<smiles>[AlH2]NC1C=CCCCCC1</smiles>

5

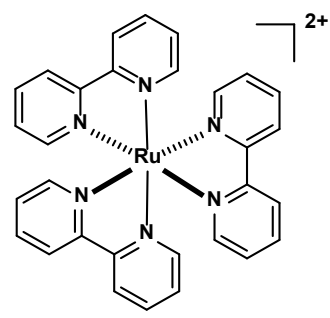

[Ru(bpy) $\left.{ }_{3}\right]\left(\mathrm{PF}_{6}\right)_{2}$

$E(R u(I I) * / R u(I))=0.77 \mathrm{~V}$

$E(R u(I I) * / R u(I I I))=-0.81 V$

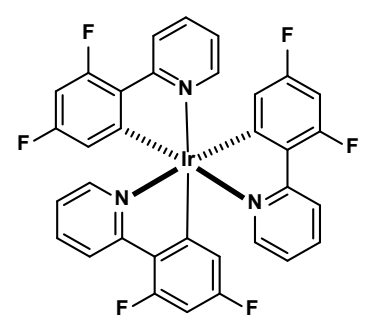<smiles>O=C(c1ccccc1)c1ccccc1</smiles>

benzophenone $E(B P * / B P)=1.28 \mathrm{~V}$ $E\left(B P^{*} / B^{+}\right)=-0.61 \mathrm{~V}$ 


\begin{tabular}{ll}
\hline photocatalyst & yield $(\%)^{\mathrm{b}}$ \\
\hline $\operatorname{Ir}\left[\mathrm{dF}\left(\mathrm{CF}_{3}\right)_{\mathrm{ppy}}\right]_{2}(\mathrm{dtbpy}) \mathrm{PF}_{6}$ & 80 \\
$\operatorname{Ir}(\mathrm{ppy})_{3}$ & 5 \\
$\left.\operatorname{Ru}(\mathrm{bpy})_{3}\right]\left(\mathrm{PF}_{6}\right)_{2}$ & 0 \\
$\operatorname{Mes}-\mathrm{Acr}{ }^{+}$ & 0 \\
$\operatorname{Ir}(\mathrm{dFppy})_{3}$ & 51 \\
benzophenone & 10 \\
none & 0 \\
\hline
\end{tabular}

a 1 ( $0.05 \mathrm{mmol})$, cyclooctene ( $0.075 \mathrm{mmol}, 1.5$ equiv.), photocatalyst ( $3 \mathrm{~mol} \%)$, TFAA (2 equiv.), HFIP (0.1 M), purple LED ( $30 \mathrm{~W}), 5{ }^{\circ} \mathrm{C} .{ }^{b} \mathrm{NMR}$ yield with $\mathrm{CH}_{2} \mathrm{Br}_{2}$ as internal standard.

Table S5. Additive reinvestigation ${ }^{a}$

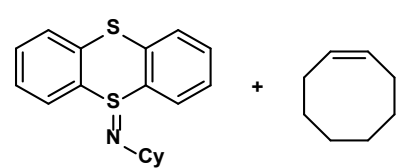

1

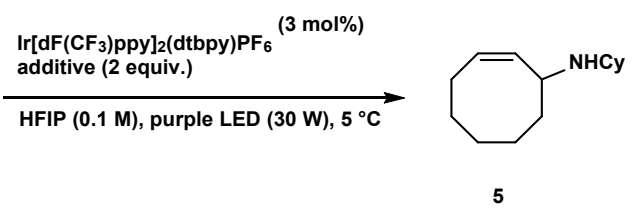

yield $(\%)^{b}$

80

TFA

50

$\mathrm{HClO}_{4}$

33

$\mathrm{Et}_{3} \mathrm{~N}$

0 
a 1 ( $0.05 \mathrm{mmol})$, cyclooctene ( $0.075 \mathrm{mmol}, 1.5$ equiv.), $\operatorname{Ir}\left[\mathrm{dF}\left(\mathrm{CF}_{3}\right) \mathrm{ppy}\right]_{2}(\mathrm{dtbpy}) \mathrm{PF}_{6}$ (3 mol\%), additive (2 equiv.), $\mathrm{HFIP}(0.1 \mathrm{M})$, purple LED $(30 \mathrm{~W}), 5{ }^{\circ} \mathrm{C} .{ }^{b} \mathrm{NMR}$ yield with $\mathrm{CH}_{2} \mathrm{Br}_{2}$ as internal standard.

Table S6. Solvent reinvestigation a

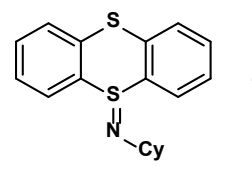

1

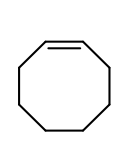

$\operatorname{Ir}_{\text {d }}\left(\mathrm{CF}_{3}\right) \mathrm{ppy}_{2}(\mathrm{dtbpy}) \mathrm{PF}_{6}(3 \mathrm{~mol} \%)$

TFAA (2 equiv.)

solvent $(0.1 \mathrm{M})$, purple LED (30 W), $5^{\circ} \mathrm{C}$

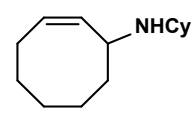

\begin{tabular}{ll}
\hline solvent & yield $(\%)^{b}$ \\
\hline HFIP & 80 \\
TFE & 65 \\
isopropanol & 35 \\
MeCN & 0 \\
DCM & 0 \\
\hline
\end{tabular}

a 1 (0.05 mmol), cyclooctene ( $0.075 \mathrm{mmol}, 1.5$ equiv.), Ir[dF(CF $)$ ppy]2(dtbpy)PF6 (3 mol\%), TFAA (2 equiv.), solvent $(0.1 \mathrm{M})$, purple LED $(30 \mathrm{~W}), 5{ }^{\circ} \mathrm{C} .{ }^{b} \mathrm{NMR}$ yield with $\mathrm{CH}_{2} \mathrm{Br}_{2}$ as internal standard.

Table S7. Additive reinvestigation ${ }^{a}$

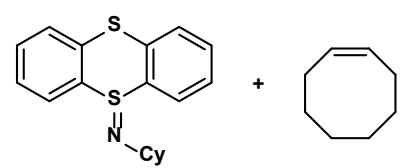

1

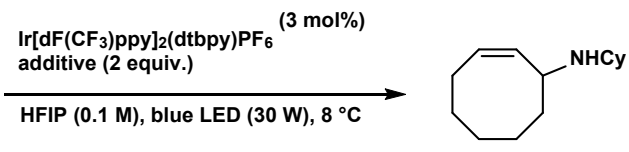

5

\begin{tabular}{ll}
\hline additive & yield $(\%)^{b}$ \\
\hline TFAA & 75 \\
TFA + $\mathrm{H}_{2} \mathrm{O}$ (1 equiv) & 28 \\
$\begin{array}{l}\text { TFA (HFIP was freshly distilled } \\
\text { from } 3 \AA \text { molecular sieves) }\end{array}$ & 64 \\
\end{tabular}

a 1 (0.05 mmol), cyclooctene (0.075 mmol, 1.5 equiv.), Ir[dF(CF3)ppy $]_{2}(\mathrm{dtbpy}) \mathrm{PF}_{6}$ (3 mol\%), additive (2 
equiv.), HFIP (0.1 M), blue LED (30 W), $8{ }^{\circ} \mathrm{C} .{ }^{b} \mathrm{NMR}$ yield with $\mathrm{CH}_{2} \mathrm{Br}_{2}$ as internal standard.

Table S8. Investigation of byproduct in different temperature ${ }^{\text {a }}$

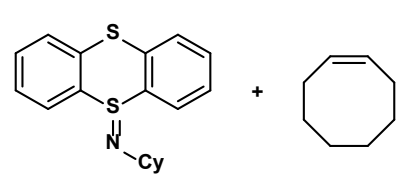

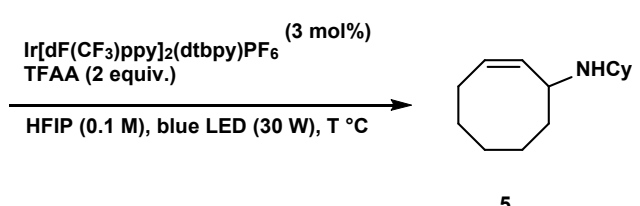

\begin{tabular}{lll}
\hline $\mathrm{T}\left({ }^{\circ} \mathrm{C}\right)$ & yield of $\mathbf{5}(\%)^{\mathrm{b}}$ & yield of cyclohexylamine $(\%)^{\mathrm{c}}$ \\
\hline 8 & 75 & 21 \\
30 & 58 & 32 \\
50 & 24 & 57
\end{tabular}

a $1(0.05 \mathrm{mmol})$, cyclooctene (0.075 mmol, 1.5 equiv.), Ir[dF(CF $)$ ppy 2 (dtbpy)PF6 (3 mol\%), TFAA (2 equiv.), HFIP (0.1 M), blue LED (30 W), $8{ }^{\circ} \mathrm{C} .{ }^{b} \mathrm{NMR}$ yield with $\mathrm{CH}_{2} \mathrm{Br}_{2}$ as internal standard. ${ }^{c} \mathrm{GC}$ yield with toluene as internal standard.

\section{Table S9. List of substrates that failed}

List of amines that failed for the synthesis of iminothianthrene:

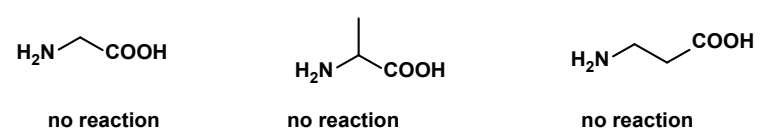

List of alkenes that failed for allylic C-H amination:

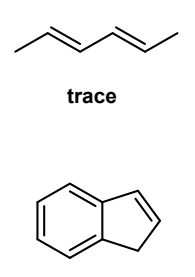

no reaction
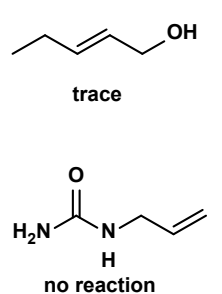
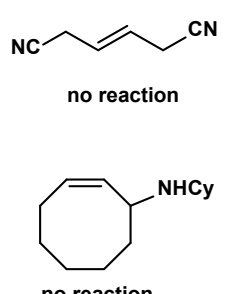

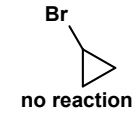

no reaction 


\section{Allylic C-H amination}

Thianthrenylidene cyclohexylamine 1

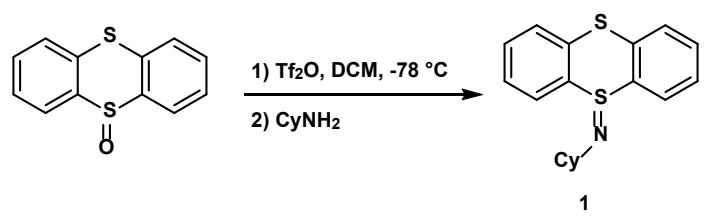

Under argon atmosphere, to a $50 \mathrm{~mL}$ two-necked round-bottomed flask were added thianthrene-S-oxide (TTO) (464 mg, $2.00 \mathrm{mmol}, 1.00$ equiv.) and $20 \mathrm{~mL}$ dry DCM. The mixture was stirred at $-78{ }^{\circ} \mathrm{C}$ followed by addition of triflic anhydride ( $353 \mu \mathrm{L}, 592 \mathrm{mg}, 2.10 \mathrm{mmol}, 1.05$ equiv.). After stirring for $30 \mathrm{~min}$, a solution of cyclohexylamine ( $570 \mu \mathrm{L}, 495 \mathrm{mg}, 5.00 \mathrm{mmol}, 2.50$ equiv.) in $\mathrm{DCM}(5 \mathrm{~mL})$ was added slowly to the reaction mixture. Then, the reaction was warmed to room temperature. Saturated sodium carbonate aqueous solution $(20 \mathrm{~mL})$ was added to the reaction mixture, which was then stirred for additional $5 \mathrm{~min}$. The resulting mixture was transferred to a separation funnel and extracted with DCM $(3 \times 20 \mathrm{~mL})$. The combined organic layers were dried over $\mathrm{Na}_{2} \mathrm{SO}_{4}$, and concentrated under reduced pressure. The obtained residue was purified by column chromatography on silica gel eluting with $\mathrm{DCM} / \mathrm{MeOH}(50 / 1-20 / 1(\mathrm{v} / \mathrm{v}))$ to afford $512 \mathrm{mg}$ of 1 as a colorless solid ( $82 \%$ yield).

Under argon atmosphere, to a $250 \mathrm{~mL}$ two-necked round-bottomed flask were added thianthrene-S-oxide (TTO) ( $2.32 \mathrm{~g}, 10.0 \mathrm{mmol}, 1.00$ equiv.) and $100 \mathrm{~mL}$ dry DCM. The mixture was stirred at $-78{ }^{\circ} \mathrm{C}$ followed by addition of triflic anhydride ( $1.70 \mathrm{~mL}, 2.94 \mathrm{~g}, 10.5 \mathrm{mmol}, 1.05$ equiv.). After stirring for $30 \mathrm{~min}$, a solution of cyclohexylamine $(2.85 \mathrm{~mL}, 2.97 \mathrm{~g}, 25.0 \mathrm{mmol}, 2.50$ equiv.) in DCM $(10 \mathrm{~mL})$ was added slowly to the reaction mixture. Then, the reaction was warmed to room temperature. Saturated sodium carbonate aqueous solution $(50 \mathrm{~mL})$ was added to the reaction mixture, which was then stirred for additional $5 \mathrm{~min}$. The resulting mixture was transferred to a separation funnel and extracted with DCM $(3 \times 50 \mathrm{~mL})$. The combined organic layers were dried over $\mathrm{Na}_{2} \mathrm{SO}_{4}$, and concentrated under reduced pressure. The obtained residue was purified by column chromatography on silica gel eluting with $\mathrm{DCM} / \mathrm{MeOH}(50 / 1-20 / 1(\mathrm{v} / \mathrm{v}))$ to afford $2.36 \mathrm{~g}$ of 1 as a colorless solid ( $75 \%$ yield).

$\mathbf{R}_{f}=0.15(\mathrm{DCM} / \mathrm{MeOH}=20 / 1(\mathrm{v} / \mathrm{v}))$.

m.m. $=106.2-108.1^{\circ} \mathrm{C}$

\section{NMR Spectroscopy:}

${ }^{1} \mathrm{H}$ NMR $\left(500 \mathrm{MHz}, \mathrm{CDCl}_{3}, 25^{\circ} \mathrm{C}, \delta\right): 8.02(\mathrm{dd}, J=7.8,1.4 \mathrm{~Hz}, 2 \mathrm{H}), 7.51(\mathrm{~d}, J=7.7 \mathrm{~Hz}, 2 \mathrm{H}), 7.47$ (t, $J=$ $7.7 \mathrm{~Hz}, 2 \mathrm{H}), 7.32(\mathrm{t}, J=7.5 \mathrm{~Hz}, 2 \mathrm{H}), 3.11-2.88(\mathrm{~m}, 1 \mathrm{H}), 2.08-1.95(\mathrm{~m}, 2 \mathrm{H}), 1.80(\mathrm{dt}, J=13.0,3.5 \mathrm{~Hz}$, $2 \mathrm{H}), 1.67-1.49(\mathrm{~m}, 3 \mathrm{H}), 1.38-1.26(\mathrm{~m}, 2 \mathrm{H}), 1.22(\mathrm{tt}, J=12.5,3.4 \mathrm{~Hz}, 1 \mathrm{H})$.

${ }^{13} \mathrm{C}$ NMR $\left(126 \mathrm{MHz}_{2} \mathrm{CDCl}_{3}, 25^{\circ} \mathrm{C}, \delta\right): 141.2,130.4,129.3,128.6,128.2,125.5,62.7,38.8,26.1,25.9$. IR (neat, thin film): $v_{\max }\left(\mathrm{cm}^{-1}\right)=3064,2917,2851,2803,1569,1434,1340,1244,1164,1148,1070,1028$, 989, 772, 769, 639 . 
HRMS-ESI(m/z) calc'd for $\mathrm{C}_{18} \mathrm{H}_{20} \mathrm{NS}_{2}[\mathrm{M}+\mathrm{H}]^{+}$, 314.1027; found, 314.1032; deviation: 1.46 ppm.

Thianthrenylidene $t$-butylamine 2

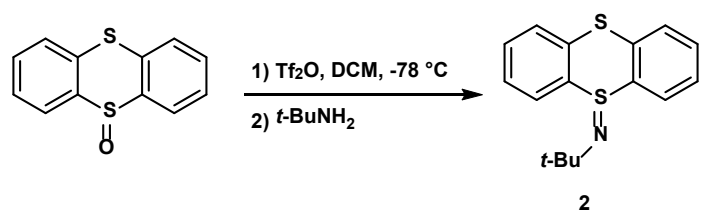

Under argon atmosphere, to a $50 \mathrm{~mL}$ two-necked round-bottomed flask were added thianthrene-S-oxide (TTO) (464 mg, $2.00 \mathrm{mmol}, 1.00$ equiv.) and $20 \mathrm{~mL}$ dry DCM. The mixture was stirred at $-78{ }^{\circ} \mathrm{C}$ followed by addition of triflic anhydride ( $353 \mu \mathrm{L}, 592 \mathrm{mg}, 2.10 \mathrm{mmol}, 1.05$ equiv.). After stirring for $30 \mathrm{~min}$, a solution of $t$ butylamine ( $525 \mu \mathrm{L}, 366 \mathrm{mg}, 5.00 \mathrm{mmol}, 2.50$ equiv.) in DCM ( $5 \mathrm{~mL}$ ) was added slowly to the reaction mixture. Then, the reaction was warmed to room temperature. Saturated sodium carbonate aqueous solution $(20 \mathrm{~mL})$ was added to the reaction mixture, which was then stirred for additional $5 \mathrm{~min}$. The resulting mixture was transferred to a separation funnel and extracted with DCM $(3 \times 20 \mathrm{~mL})$. The combined organic layers were dried over $\mathrm{Na}_{2} \mathrm{SO}_{4}$, and concentrated under reduced pressure. The obtained residue was purified by column chromatography on silica gel eluting with $\mathrm{DCM} / \mathrm{MeOH}(50 / 1-20 / 1(\mathrm{v} / \mathrm{v}))$ to afford $440 \mathrm{mg}$ of 2 as a colorless solid ( $76 \%$ yield).

$\boldsymbol{R}_{\boldsymbol{f}}=0.20(\mathrm{DCM} / \mathrm{MeOH}=20 / 1(\mathrm{v} / \mathrm{v}))$.

m.m. $=138.1-139.3^{\circ} \mathrm{C}$

\section{NMR Spectroscopy:}

${ }^{1} \mathrm{H}$ NMR $\left(500 \mathrm{MHz}, \mathrm{CDCl}_{3}, 25^{\circ} \mathrm{C}, \delta\right): 8.02(\mathrm{dd}, J=7.8,1.4 \mathrm{~Hz}, 2 \mathrm{H}), 7.53(\mathrm{dd}, J=7.6,1.3 \mathrm{~Hz}, 2 \mathrm{H}), 7.49$ (td, $J=7.6,1.3 \mathrm{~Hz}, 2 \mathrm{H}), 7.37-7.31(\mathrm{~m}, 2 \mathrm{H}), 1.44(\mathrm{~s}, 9 \mathrm{H})$.

${ }^{13} \mathrm{C}$ NMR $\left(126 \mathrm{MHz} \mathrm{CDCl}_{3}, 25^{\circ} \mathrm{C}, \delta\right): 141.6,130.3,129.2,128.8,128.3,125.1,54.5,33.5$.

IR (neat, thin film): $V_{\max }\left(\mathrm{cm}^{-1}\right)=3044,2948,2897,2853,1565,1436,1353,1241,1184,1009,840,749$, $734,711$.

HRMS-ESI(m/z) calc'd for $\mathrm{C}_{16} \mathrm{H}_{18} \mathrm{NS}_{2}[\mathrm{M}+\mathrm{H}]^{+}$, 288.0873; found, 288.0875; deviation: 0.62 ppm.

Thianthrenylidene $n$-propylamine 3

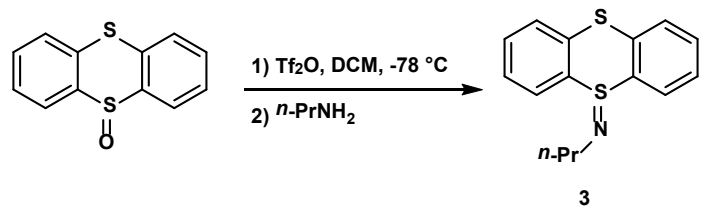

Under argon atmosphere, to a $50 \mathrm{~mL}$ two-necked round-bottomed flask were added thianthrene-S-oxide (TTO) (464 mg, $2.00 \mathrm{mmol}, 1.00$ equiv.) and $20 \mathrm{~mL}$ dry DCM. The mixture was stirred at $-78{ }^{\circ} \mathrm{C}$ followed by addition of triflic anhydride ( $353 \mu \mathrm{L}, 592 \mathrm{mg}, 2.10 \mathrm{mmol}, 1.05$ equiv.). After stirring for $30 \mathrm{~min}$, a solution of $n$ - 
propylamine ( $411 \mu \mathrm{L}, 296 \mathrm{mg}, 5.00 \mathrm{mmol}, 2.50$ equiv.) in $\mathrm{DCM}(5 \mathrm{~mL})$ was added slowly to the reaction mixture. Then, the reaction was warmed to room temperature. Saturated sodium carbonate aqueous solution $(20 \mathrm{~mL})$ was added to the reaction mixture, which was then stirred for additional $5 \mathrm{~min}$. The resulting mixture was transferred to a separation funnel and extracted with DCM $(3 \times 20 \mathrm{~mL})$. The combined organic layers were dried over $\mathrm{Na}_{2} \mathrm{SO}_{4}$, and concentrated under reduced pressure. The obtained residue was purified by column chromatography on silica gel eluting with $\mathrm{DCM} / \mathrm{MeOH}(50 / 1-20 / 1(\mathrm{v} / \mathrm{v}))$ to afford $380 \mathrm{mg}$ of 3 as a colorless oil (69\% yield).

$\mathbf{R}_{\boldsymbol{f}}=0.15(\mathrm{DCM} / \mathrm{MeOH}=20 / 1(\mathrm{v} / \mathrm{v}))$.

\section{NMR Spectroscopy:}

${ }^{1} \mathrm{H}$ NMR $\left(500 \mathrm{MHz}, \mathrm{CDCl}_{3}, 25^{\circ} \mathrm{C}, \delta\right): 7.91$ (dt, $\left.J=7.7,1.3 \mathrm{~Hz}, 2 \mathrm{H}\right), 7.55-7.50(\mathrm{~m}, 2 \mathrm{H}), 7.49-7.42(\mathrm{~m}$, $2 \mathrm{H}), 7.41-7.34(\mathrm{~m}, 2 \mathrm{H}), 2.94(\mathrm{t}, J=7.3 \mathrm{~Hz}, 2 \mathrm{H}), 1.74-1.61(\mathrm{~m}, 2 \mathrm{H}), 0.94(\mathrm{td}, J=7.4,1.2 \mathrm{~Hz}, 3 \mathrm{H})$.

${ }^{13} \mathrm{C}$ NMR $\left(126 \mathrm{MHz}, \mathrm{CDCl}_{3}, 25{ }^{\circ} \mathrm{C}, \delta\right): 136.5,131.2,129.8,128.4,127.9,127.7,52.1,27.1,12.2$.

IR (neat, thin film): $v_{\max }\left(\mathrm{cm}^{-1}\right)=3053,2953,2925,2871,2829,1567,1432,1370,1246,1084,1021,839$, 747.

HRMS-ESI(m/z) calc'd for $\mathrm{C}_{15} \mathrm{H}_{16} \mathrm{NS}_{2}[\mathrm{M}+\mathrm{H}]^{+}, 274.0716$; found, 274.0719; deviation: 0.98 ppm.

Thianthrenylidene 1-adamantylamine 4

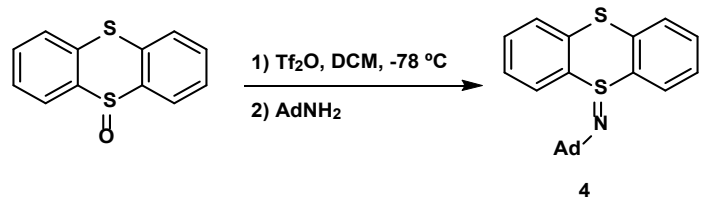

Under argon atmosphere, to a $50 \mathrm{~mL}$ two-necked round-bottomed flask were added thianthrene-S-oxide (TTO) (464 mg, $2.00 \mathrm{mmol}, 1.00$ equiv.) and $20 \mathrm{~mL}$ dry DCM. The mixture was stirred at $-78{ }^{\circ} \mathrm{C}$ followed by addition of triflic anhydride ( $353 \mu \mathrm{L}, 592 \mathrm{mg}, 2.10 \mathrm{mmol}, 1.05$ equiv.). After stirring for $30 \mathrm{~min}$, a solution of 1adamantylamine (756 mg, $5.00 \mathrm{mmol}, 2.50$ equiv.) in DCM $(5 \mathrm{~mL})$ was added slowly to the reaction mixture. Then, the reaction was warmed to room temperature. Saturated sodium carbonate aqueous solution $(20 \mathrm{~mL})$ was added to the reaction mixture, which was then stirred for additional $5 \mathrm{~min}$. The resulting mixture was transferred to a separation funnel and extracted with DCM $(3 \times 20 \mathrm{~mL})$. The combined organic layers were dried over $\mathrm{Na}_{2} \mathrm{SO}_{4}$, and concentrated under reduced pressure. The obtained residue was purified by column chromatography on silica gel eluting with $\mathrm{DCM} / \mathrm{MeOH}(50 / 1-30 / 1(\mathrm{v} / \mathrm{v}))$ to afford $445 \mathrm{mg}$ of 4 as a colorless solid $(61 \%$ yield).

$\mathbf{R}_{\boldsymbol{f}}=0.30(\mathrm{DCM} / \mathrm{MeOH}=20 / 1(\mathrm{v} / \mathrm{v}))$.

m.m. $=165.7-166.9^{\circ} \mathrm{C}$

\section{NMR Spectroscopy:}

${ }^{1} \mathrm{H}$ NMR $\left(500 \mathrm{MHz}, \mathrm{CDCl}_{3}, 25^{\circ} \mathrm{C}, \delta\right): 8.07$ (dd, $\left.J=7.8,1.4 \mathrm{~Hz}, 2 \mathrm{H}\right), 7.54(\mathrm{dd}, J=7.6,1.2 \mathrm{~Hz}, 2 \mathrm{H}), 7.50$ 
(td, $J=7.6,1.2 \mathrm{~Hz}, 2 \mathrm{H}), 7.35(\mathrm{td}, J=7.5,1.4 \mathrm{~Hz}, 2 \mathrm{H}), 2.19-2.04(\mathrm{~m}, 3 \mathrm{H}), 1.98(\mathrm{~d}, J=2.9 \mathrm{~Hz}, 6 \mathrm{H}), 1.69$ (t, $J=3.1 \mathrm{~Hz}, 6 \mathrm{H})$.

${ }^{13} \mathrm{C}$ NMR $\left(126 \mathrm{MHz}, \mathrm{CDCl}_{3}, 25^{\circ} \mathrm{C}, \delta\right): 142.0,130.4,129.2,128.7,128.3,125.3,54.2,47.4,36.7,30.7$. IR (neat, thin film): $v_{\max }\left(\mathrm{cm}^{-1}\right)=3048,2900,2849,1558,1433,1344,1234,1113,1090,1003,858,758$, $741,605$.

HRMS-ESI(m/z) calc'd for $\mathrm{C}_{22} \mathrm{H}_{24} \mathrm{NS}_{2}[\mathrm{M}+\mathrm{H}]^{+}, 366.1343$; found, 366.1345; deviation: $0.52 \mathrm{ppm}$.

Thianthrenylidene neopentylamine S1

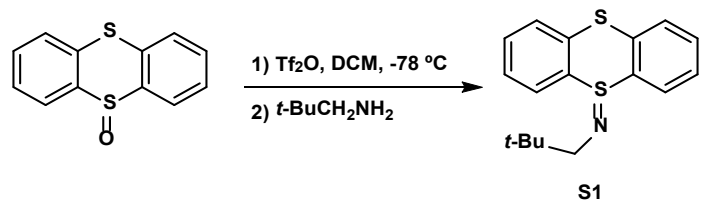

Under argon atmosphere, to a $50 \mathrm{~mL}$ two-necked round-bottomed flask were added thianthrene-S-oxide (TTO) (464 mg, $2.00 \mathrm{mmol}, 1.00$ equiv.) and $20 \mathrm{~mL}$ dry DCM. The mixture was stirred at $-78{ }^{\circ} \mathrm{C}$ followed by addition of triflic anhydride ( $353 \mu \mathrm{L}, 592 \mathrm{mg}, 2.10 \mathrm{mmol}, 1.05$ equiv.). After stirring for $30 \mathrm{~min}$, a solution of neopentylamine ( $585 \mu \mathrm{L}, 436 \mathrm{mg}, 5.00 \mathrm{mmol}, 2.50$ equiv.) in DCM $(5 \mathrm{~mL})$ was added slowly to the reaction mixture. Then, the reaction was warmed to room temperature. Saturated sodium carbonate aqueous solution $(20 \mathrm{~mL})$ was added to the reaction mixture, which was then stirred for additional $5 \mathrm{~min}$. The resulting mixture was transferred to a separation funnel and extracted with DCM $(3 \times 20 \mathrm{~mL})$. The combined organic layers were dried over $\mathrm{Na}_{2} \mathrm{SO}_{4}$, and concentrated under reduced pressure. The obtained residue was purified by column chromatography on silica gel eluting with $\mathrm{DCM} / \mathrm{MeOH}(50 / 1-20 / 1(\mathrm{v} / \mathrm{v}))$ to afford $375 \mathrm{mg}$ of $\mathbf{S} 1$ as a colorless oil (62\% yield).

$\mathbf{R}_{\boldsymbol{f}}=0.20(\mathrm{DCM} / \mathrm{MeOH}=20 / 1(\mathrm{v} / \mathrm{v}))$.

\section{NMR Spectroscopy:}

${ }^{1} \mathrm{H}$ NMR $\left(500 \mathrm{MHz}, \mathrm{CDCl}_{3}, 25^{\circ} \mathrm{C}, \delta\right): 7.99-7.90(\mathrm{~m}, 2 \mathrm{H}), 7.52(\mathrm{dd}, J=7.7,1.2 \mathrm{~Hz}, 2 \mathrm{H}), 7.47$ (td, $J=$ 7.6, $1.3 \mathrm{~Hz}, 2 \mathrm{H}), 7.38$ (td, $J=7.5,1.5 \mathrm{~Hz}, 2 \mathrm{H}), 2.80$ (s, 2H), $0.99(\mathrm{~s}, 9 \mathrm{H})$.

${ }^{13} \mathrm{C}$ NMR $\left(126 \mathrm{MHz}, \mathrm{CDCl}_{3}, 25{ }^{\circ} \mathrm{C}, \delta\right): 137.0,131.1,129.8,128.4,127.9,127.6,62.6,33.4,27.9$.

IR (neat, thin film): $V_{\max }\left(\mathrm{cm}^{-1}\right)=3052,2938,2852,2827,1567,1431,1355,1251,1119,1023,855,754$, 732.

HRMS-ESI(m/z) calc'd for $\mathrm{C}_{17} \mathrm{H}_{20} \mathrm{NS}_{2}[\mathrm{M}+\mathrm{H}]^{+}$, 302.1029; found, 302.1032; deviation: 0.99 ppm. 
2-(Thianthrenylidene)aminoethyl pivalate S2

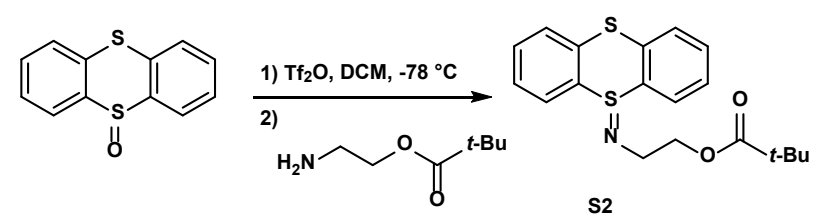

Under argon atmosphere, to a $50 \mathrm{~mL}$ two-necked round-bottomed flask were added thianthrene-S-oxide (TTO) (464 mg, $2.00 \mathrm{mmol}, 1.00$ equiv.) and $20 \mathrm{~mL}$ dry DCM. The mixture was stirred at $-78{ }^{\circ} \mathrm{C}$ followed by addition of triflic anhydride ( $353 \mu \mathrm{L}, 592 \mathrm{mg}, 2.10 \mathrm{mmol}, 1.05$ equiv.). After stirring for $30 \mathrm{~min}$, a solution of 2aminoethyl pivalate $(750 \mu \mathrm{L}, 726 \mathrm{mg}, 5.00 \mathrm{mmol}, 2.50$ equiv.) in DCM $(5 \mathrm{~mL})$ was added slowly to the reaction mixture. Then, the reaction was warmed to room temperature. Saturated sodium carbonate aqueous solution $(20 \mathrm{~mL})$ was added to the reaction mixture, which was then stirred for additional $5 \mathrm{~min}$. The resulting mixture was transferred to a separation funnel and extracted with DCM $(3 \times 20 \mathrm{~mL})$. The combined organic layers were dried over $\mathrm{Na}_{2} \mathrm{SO}_{4}$, and concentrated under reduced pressure. The obtained residue was purified by column chromatography on silica gel eluting with $\mathrm{DCM} / \mathrm{MeOH}(50 / 1-30 / 1(\mathrm{v} / \mathrm{v}))$ to afford $295 \mathrm{mg}$ of $\mathbf{S} 2$ as a colorless solid ( $41 \%$ yield).

$\mathbf{R}_{\boldsymbol{f}}=0.25(\mathrm{DCM} / \mathrm{MeOH}=20 / 1(\mathrm{v} / \mathrm{v}))$.

m.m. $=96.7-97.6^{\circ} \mathrm{C}$

\section{NMR Spectroscopy:}

${ }^{1} \mathrm{H}$ NMR $\left(500 \mathrm{MHz}, \mathrm{CDCl}_{3}, 25^{\circ} \mathrm{C}, \delta\right): 8.04$ (dd, $\left.J=7.9,1.4 \mathrm{~Hz}, 2 \mathrm{H}\right), 7.59$ (dd, $\left.J=7.7,1.3 \mathrm{~Hz}, 2 \mathrm{H}\right), 7.53$ (td, $J=7.6,1.3 \mathrm{~Hz}, 2 \mathrm{H}), 7.45$ (td, $J=7.5,1.4 \mathrm{~Hz}, 2 \mathrm{H}), 4.30$ (t, $J=5.5 \mathrm{~Hz}, 2 \mathrm{H}), 3.42(\mathrm{t}, J=5.5 \mathrm{~Hz}, 2 \mathrm{H}$ ), $1.08(\mathrm{~s}, 9 \mathrm{H})$.

${ }^{13} \mathrm{C}$ NMR $\left(126 \mathrm{MHz}, \mathrm{CDCl}_{3}, 25{ }^{\circ} \mathrm{C}, \delta\right): 178.7,135.3,131.3,130.6,128.9,128.5,127.5,66.7,49.2,38.8$, 27.2.

IR (neat, thin film): $V_{\max }\left(\mathrm{cm}^{-1}\right)=3054,2941,2857,1732,1555,1433,1332,1289,1139,1036,845,742$, 722.

HRMS-ESI(m/z) calc'd for $\mathrm{C}_{19} \mathrm{H}_{22} \mathrm{NO}_{2} \mathrm{~S}_{2}[\mathrm{M}+\mathrm{H}]^{+}$, 360.1084; found, 360.1086; deviation: 0.77 ppm.

Ethyl 2-(thianthrenylidene)amino-2-methylpropanoate S3

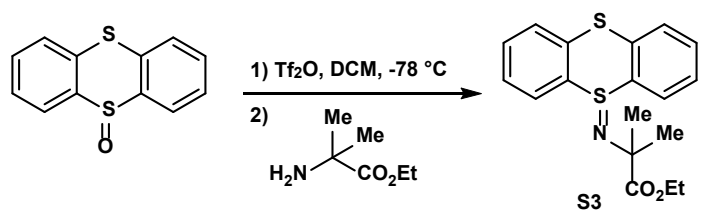

Under argon atmosphere, to a $50 \mathrm{~mL}$ two-necked round-bottomed flask were added thianthrene-S-oxide (TTO) (464 mg, $2.00 \mathrm{mmol}, 1.00$ equiv.) and $20 \mathrm{~mL}$ dry DCM. The mixture was stirred at $-78{ }^{\circ} \mathrm{C}$ followed by addition of triflic anhydride ( $353 \mu \mathrm{L}, 592 \mathrm{mg}, 2.10 \mathrm{mmol}, 1.05$ equiv.). After stirring for $30 \mathrm{~min}$, a solution of 
ethyl 2-amino-2-methylpropanoate $(680 \mu \mathrm{L}, 656 \mathrm{mg}, 5.00 \mathrm{mmol}, 2.50$ equiv.) in DCM (5 mL) was added slowly to the reaction mixture. Then, the reaction was warmed to room temperature. Saturated sodium carbonate aqueous solution $(20 \mathrm{~mL})$ was added to the reaction mixture, which was then stirred for additional $5 \mathrm{~min}$. The resulting mixture was transferred to a separation funnel and extracted with $\mathrm{DCM}(3 \times 20 \mathrm{~mL})$. The combined organic layers were dried over $\mathrm{Na}_{2} \mathrm{SO}_{4}$, and concentrated under reduced pressure. The obtained residue was purified by column chromatography on silica gel eluting with $\mathrm{DCM} / \mathrm{MeOH}(50 / 1-30 / 1(\mathrm{v} / \mathrm{v}))$ to afford $309 \mathrm{mg}$ of $\mathbf{S} \mathbf{3}$ as a colorless solid (45\% yield).

$\mathbf{R}_{\boldsymbol{f}}=0.25(\mathrm{DCM} / \mathrm{MeOH}=20 / 1(\mathrm{v} / \mathrm{v}))$.

m.m. $=73.8-74.6^{\circ} \mathrm{C}$

\section{NMR Spectroscopy:}

${ }^{1} \mathrm{H}$ NMR $\left(500 \mathrm{MHz}, \mathrm{CDCl}_{3}, 25{ }^{\circ} \mathrm{C}, \delta\right): 8.05(\mathrm{dd}, J=7.9,1.3 \mathrm{~Hz}, 2 \mathrm{H}), 7.54(\mathrm{dd}, J=7.6,1.4 \mathrm{~Hz}, 2 \mathrm{H}), 7.49$ (tt, $J=7.8,1.4 \mathrm{~Hz}, 2 \mathrm{H}), 7.34$ (tt, $J=7.5,1.7 \mathrm{~Hz}, 2 \mathrm{H}$ ), 4.10 (qd, $J=7.1,1.3 \mathrm{~Hz}, 2 \mathrm{H}), 1.69$ (d, $J=1.1 \mathrm{~Hz}$, $6 \mathrm{H}), 1.15$ (td, $J=7.1,1.4 \mathrm{~Hz}, 3 \mathrm{H})$.

${ }^{13} \mathrm{C}$ NMR $\left(126 \mathrm{MHz}, \mathrm{CDCl}_{3}, 2{ }^{\circ} \mathrm{C}, \delta\right): 177.9,140.1,130.4,129.4,128.9,128.3,125.6,61.5,60.9,29.5$, 14.2 .

IR (neat, thin film): $v_{\max }\left(\mathrm{cm}^{-1}\right)=3053,2981,2921,1725,1554,1432,1368,1245,1174,1157,1097,1026$, $868,748,637$.

HRMS-ESI(m/z) calc'd for $\mathrm{C}_{18} \mathrm{H}_{20} \mathrm{NO}_{2} \mathrm{~S}_{2}[\mathrm{M}+\mathrm{H}]^{+}, 346.0927$; found, 346.0930; deviation: 0.81 ppm.

Thianthrenylidene a-methylbenzylamine S4

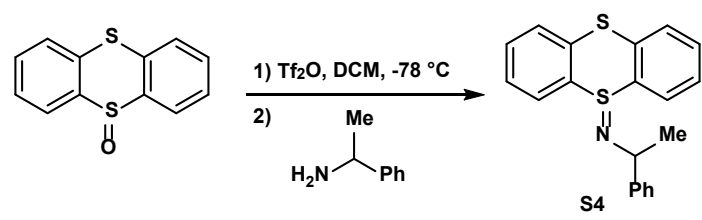

Under argon atmosphere, to a $50 \mathrm{~mL}$ two-necked round-bottomed flask were added thianthrene-S-oxide (TTO) (464 mg, $2.00 \mathrm{mmol}, 1.00$ equiv.) and $20 \mathrm{~mL}$ dry DCM. The mixture was stirred at $-78{ }^{\circ} \mathrm{C}$ followed by addition of triflic anhydride ( $353 \mu \mathrm{L}, 592 \mathrm{mg}, 2.10 \mathrm{mmol}, 1.05$ equiv.). After stirring for $30 \mathrm{~min}$, to the reaction mixture was added DCM $(5 \mathrm{~mL})$ solution of $\alpha$-methylbenzylamine $(645 \mu \mathrm{L}, 606 \mathrm{mg}, 5.00 \mathrm{mmol}, 2.50$ equiv.) slowly. Then, the reaction was warmed to room temperature. Saturated sodium carbonate aqueous solution $(20 \mathrm{~mL})$ was added to the reaction mixture, which was then stirred for additional $5 \mathrm{~min}$. The resulting mixture was transferred to a separation funnel and extracted with DCM $(3 \times 20 \mathrm{~mL})$. The combined organic layers were dried over $\mathrm{Na}_{2} \mathrm{SO}_{4}$, and concentrated under reduced pressure. The obtained residue was purified by column chromatography on silica gel eluting with $\mathrm{DCM} / \mathrm{MeOH}(50 / 1-20 / 1(\mathrm{v} / \mathrm{v}))$ to afford $378 \mathrm{mg}$ of $\mathbf{S} 4$ as a colorless solid ( $56 \%$ yield).

$\boldsymbol{R}_{\boldsymbol{f}}=0.20(\mathrm{DCM} / \mathrm{MeOH}=20 / 1(\mathrm{v} / \mathrm{v}))$. 
m.m. $=83.7-84.4^{\circ} \mathrm{C}$

\section{NMR Spectroscopy:}

${ }^{1} \mathrm{H}$ NMR $\left(500 \mathrm{MHz}, \mathrm{CDCl}_{3}, 25^{\circ} \mathrm{C}, \delta\right): 8.02(\mathrm{dd}, J=7.8,1.4 \mathrm{~Hz}, 1 \mathrm{H}), 7.73(\mathrm{dd}, J=7.7,1.5 \mathrm{~Hz}, 1 \mathrm{H}), 7.54-$ $7.44(\mathrm{~m}, 5 \mathrm{H}), 7.39-7.27(\mathrm{~m}, 5 \mathrm{H}), 7.22-7.16(\mathrm{~m}, 1 \mathrm{H}), 4.41$ (q, $J=6.7 \mathrm{~Hz}, 1 \mathrm{H}), 1.72(\mathrm{~d}, J=6.7 \mathrm{~Hz}, 3 \mathrm{H})$.

${ }^{13} \mathrm{C}$ NMR $\left(126 \mathrm{MHz}, \mathrm{CDCl}_{3}, 25^{\circ} \mathrm{C}, \delta\right): 149.0,139.6,130.8,130.7,129.6,129.5,128.8,128.6,128.5$, $128.4,128.2,127.8,126.6,126.5,126.5,126.1,62.8,27.5$.

IR (neat, thin film): $v_{\max }\left(\mathrm{cm}^{-1}\right)=3048,2986,2934,2855,1563,1438,1333,1264,1108,1020,887,757$, 743.

HRMS-ESI(m/z) calc'd for $\mathrm{C}_{20} \mathrm{H}_{18} \mathrm{NS}_{2}[\mathrm{M}+\mathrm{H}]^{+}, 336.0872$; found, 336.0875; deviation: $0.98 \mathrm{ppm}$.

3-(Thianthrenylidene)aminopropan-1-ol S5

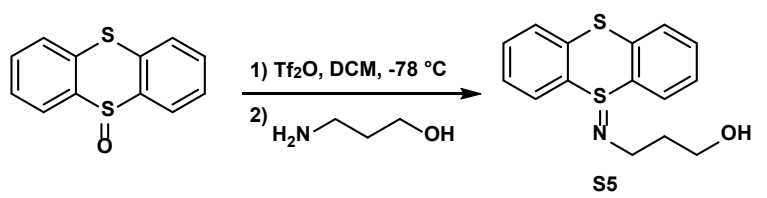

Under argon atmosphere, to a $50 \mathrm{~mL}$ two-necked round-bottomed flask were added thianthrene-S-oxide (TTO) (464 mg, $2.00 \mathrm{mmol}, 1.00$ equiv.) and $20 \mathrm{~mL}$ dry DCM. The mixture was stirred at $-78{ }^{\circ} \mathrm{C}$ followed by addition of triflic anhydride ( $353 \mu \mathrm{L}, 592 \mathrm{mg}, 2.10 \mathrm{mmol}, 1.05$ equiv.). After stirring for $30 \mathrm{~min}$, to the reaction mixture was added DCM (5 mL) solution of 3-aminopropan-1-ol (383 $\mu \mathrm{L}, 376 \mathrm{mg}, 5.00 \mathrm{mmol}, 2.50$ equiv.) slowly. Then, the reaction was warmed to room temperature. Saturated sodium carbonate aqueous solution $(20 \mathrm{~mL})$ was added to the reaction mixture, which was then stirred for additional $5 \mathrm{~min}$. The resulting mixture was transferred to a separation funnel and extracted with DCM $(3 \times 20 \mathrm{~mL})$. The combined organic layers were dried over $\mathrm{Na}_{2} \mathrm{SO}_{4}$, and concentrated under reduced pressure. The obtained residue was purified by column chromatography on silica gel eluting with $\mathrm{DCM} / \mathrm{MeOH}(30 / 1-10 / 1(\mathrm{v} / \mathrm{v}))$ to afford $210 \mathrm{mg}$ of $\mathbf{S 5}$ as a colorless liquid (36\% yield).

$\boldsymbol{R}_{\boldsymbol{f}}=0.20(\mathrm{DCM} / \mathrm{MeOH}=10 / 1(\mathrm{v} / \mathrm{v}))$.

\section{NMR Spectroscopy:}

${ }^{1} \mathrm{H}$ NMR $\left(500 \mathrm{MHz}, \mathrm{CDCl}_{3}, 25^{\circ} \mathrm{C}, \delta\right): 7.92(\mathrm{dd}, J=7.8,1.4 \mathrm{~Hz}, 2 \mathrm{H}), 7.52(\mathrm{dd}, J=7.6,1.4 \mathrm{~Hz}, 2 \mathrm{H}), 7.47$ (td, $J=7.5,1.3 \mathrm{~Hz}, 2 \mathrm{H}), 7.41(\mathrm{td}, J=7.5,1.4 \mathrm{~Hz}, 2 \mathrm{H}), 4.59(\mathrm{br}, 1 \mathrm{H}), 3.84(\mathrm{t}, J=5.4 \mathrm{~Hz}, 2 \mathrm{H}), 3.10(\mathrm{t}, J=$ $5.9 \mathrm{~Hz}, 2 \mathrm{H}), 1.81(\mathrm{dt}, J=10.5,5.7 \mathrm{~Hz}, 2 \mathrm{H})$.

${ }^{13} \mathrm{C}$ NMR $\left(126 \mathrm{MHz}, \mathrm{CDCl}_{3}, 25{ }^{\circ} \mathrm{C}, \delta\right): 133.5,131.8,130.5,128.5,128.3,128.2,63.7,48.4,33.9$.

IR (neat, thin film): $V_{\max }\left(\mathrm{cm}^{-1}\right)=3539,3137,3096,2929,2866,1575,1441,1356,1281,1228,1163,1094$, 1026, 977, 820, 749.

HRMS-ESI(m/z) calc'd for $\mathrm{C}_{15} \mathrm{H}_{16} \mathrm{NOS}_{2}[\mathrm{M}+\mathrm{H}]^{+}$, 290.0666; found, 290.0668; deviation: 0.57 ppm. 
(Z)-N-Cyclohexyl-2-cyclooctenamine 5

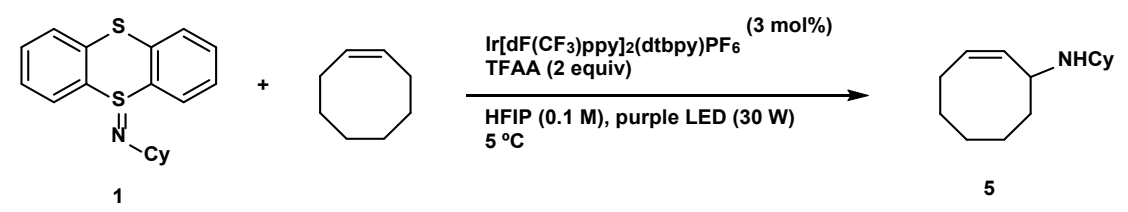

Under nitrogen atmosphere, to a 4-mL borosilicate vial equipped with a magnetic stir bar were added thianthrenylidene cyclohexylamine 1 (31.3 mg, $0.100 \mathrm{mmol}, 1.00$ equiv.), cis-cyclooctene (20.0 $\mu \mathrm{L}, 16.5 \mathrm{mg}$, $0.150 \mathrm{mmol}, 1.50$ equiv.), $\operatorname{Ir}\left[\mathrm{dF}\left(\mathrm{CF}_{3}\right) \mathrm{ppy}\right]_{2}(\mathrm{dtbpy}) \mathrm{PF}_{6}(3.3 \mathrm{mg}, 3.0 \mu \mathrm{mol}, 3.0 \mathrm{~mol} \%), \mathrm{HFIP}(1 \mathrm{~mL}, \mathrm{c}=0.1 \mathrm{M})$, and trifluoroacetic anhydride ( $28 \mu \mathrm{L}, 42 \mathrm{mg}, 0.20 \mathrm{mmol}, 2.0$ equiv.). The vial was sealed with a septum-cap and irradiated for $3 \mathrm{~h}$ at $5{ }^{\circ} \mathrm{C}$ using a purple LED (30 W). Then, the reaction mixture was concentrated. The residue was dissolved in DCM $(5 \mathrm{~mL})$ and washed with saturated sodium carbonate aqueous solution $(5 \mathrm{~mL})$. The aqueous phase was extracted with DCM $(2 \times 5 \mathrm{~mL})$. The organic phase was dried over $\mathrm{Na}_{2} \mathrm{SO}_{4}$ and the solvent was removed under reduced pressure. The residue was purified by chromatography on silica gel eluting with $\mathrm{CHCl}_{3} / \mathrm{MeOH}(50 / 1-30 / 1(\mathrm{v} / \mathrm{v}))$ to afford $16.0 \mathrm{mg}$ of 5 as a colorless oil $(77 \%$ yield, r.r. $=10: 1)$. Under nitrogen atmosphere, to a 4-mL borosilicate vial equipped with a magnetic stir bar were added thianthrenylidene cyclohexylamine 1 (31.3 mg, $0.100 \mathrm{mmol}, 1.00$ equiv.), cis-cyclooctene (13.3 $\mu \mathrm{L}, 11.0 \mathrm{mg}$, $0.100 \mathrm{mmol}, 1.00$ equiv.), $\operatorname{Ir}\left[\mathrm{dF}\left(\mathrm{CF}_{3}\right) \mathrm{ppy}_{2}(\mathrm{dtbpy}) \mathrm{PF}_{6}(3.3 \mathrm{mg}, 3.0 \mu \mathrm{mol}, 3.0 \mathrm{~mol} \%), \mathrm{HFIP}(1 \mathrm{~mL}, \mathrm{c}=0.1 \mathrm{M})\right.$, and trifluoroacetic anhydride ( $28 \mu \mathrm{L}, 42 \mathrm{mg}, 0.20 \mathrm{mmol}, 2.0$ equiv.). The vial was sealed with a septum-cap and irradiated for $3 \mathrm{~h}$ at $5{ }^{\circ} \mathrm{C}$ using a purple LED $(30 \mathrm{~W})$. Then, the reaction mixture was concentrated. The residue was dissolved in $\mathrm{DCM}(5 \mathrm{~mL})$ and washed with saturated sodium carbonate aqueous solution $(5 \mathrm{~mL})$. The aqueous phase was extracted with DCM $(2 \times 5 \mathrm{~mL})$. The organic phase was dried over $\mathrm{Na}_{2} \mathrm{SO}_{4}$ and the solvent was removed under reduced pressure. The residue was purified by chromatography on silica gel eluting with $\mathrm{CHCl}_{3} / \mathrm{MeOH}(50 / 1-30 / 1(\mathrm{v} / \mathrm{v}))$ to afford $12.6 \mathrm{mg}$ of 5 as a colorless oil $(61 \%$ yield, r.r. $=10: 1)$.

$\boldsymbol{R}_{\boldsymbol{f}}=0.25\left(\mathrm{CHCl}_{3} / \mathrm{MeOH}=10 / 1(\mathrm{v} / \mathrm{v})\right)$.

\section{NMR Spectroscopy:}

${ }^{1} \mathrm{H}$ NMR $\left(500 \mathrm{MHz}, \mathrm{CDCl}_{3}, 25^{\circ} \mathrm{C}, \delta\right): 5.65$ (dtd, $\left.J=10.6,7.4,1.5 \mathrm{~Hz}, 1 \mathrm{H}\right), 5.30$ (ddd, $J=10.6,7.9,1.5$ $\mathrm{Hz}, 1 \mathrm{H}), 3.82-3.70(\mathrm{~m}, 1 \mathrm{H}), 2.56-2.45(\mathrm{~m}, 1 \mathrm{H}), 2.25-2.12(\mathrm{~m}, 1 \mathrm{H}), 2.12-2.04(\mathrm{~m}, 1 \mathrm{H}), 1.98-1.86$ $(\mathrm{m}, 1 \mathrm{H}), 1.86-1.75(\mathrm{~m}, 1 \mathrm{H}), 1.76-1.63(\mathrm{~m}, 4 \mathrm{H}), 1.62-1.56(\mathrm{~m}, 1 \mathrm{H}), 1.55-1.47(\mathrm{~m}, 1 \mathrm{H}), 1.44-0.89$ $(\mathrm{m}, 10 \mathrm{H})$.

${ }^{13} \mathrm{C}$ NMR $\left(126 \mathrm{MHz}_{2} \mathrm{CDCl}_{3}, 25^{\circ} \mathrm{C}, \delta\right): 136.1,129.6,54.4,51.7,37.5,35.0,33.6,29.7,27.1,26.9,26.4$, 25.5, 25.3, 24.9.

HRMS-EI(m/z) calc'd for $\mathrm{C}_{14} \mathrm{H}_{25} \mathrm{~N}$ [M] ${ }^{+}, 207.1982$; found, 207.1981; deviation: -0.3 ppm.

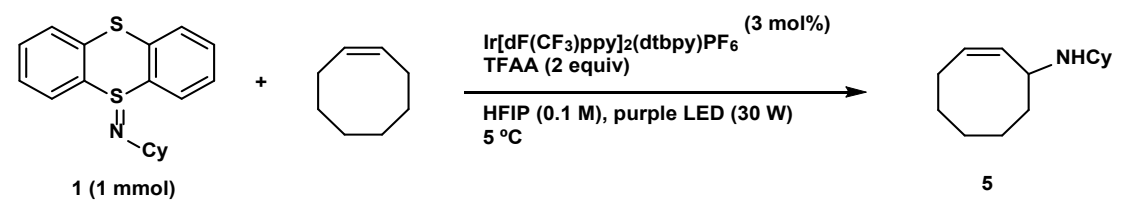


Under nitrogen atmosphere, to a 4-mL borosilicate vial equipped with a magnetic stir bar were added thianthrenylidene cyclohexylamine 1 (313 mg, 1.00 mmol, 1.00 equiv.), cis-cyclooctene (200 $\mu \mathrm{L}, 165 \mathrm{mg}$, $1.50 \mathrm{mmol}, 1.50$ equiv.), $\operatorname{Ir}\left[\mathrm{dF}\left(\mathrm{CF}_{3}\right) \text { ppy }\right]_{2}$ (dtbpy)PF 6 (33 mg, $\left.30 \mu \mathrm{mol}, 3.0 \mathrm{~mol} \%\right), \mathrm{HFIP}(10 \mathrm{~mL}, \mathrm{c}=0.1 \mathrm{M})$, and trifluoroacetic anhydride ( $280 \mu \mathrm{L}, 420 \mathrm{mg}, 2.00 \mathrm{mmol}, 2.00$ equiv.). The vial was sealed with a septum-cap and irradiated for $24 \mathrm{~h}$ at $5{ }^{\circ} \mathrm{C}$ using a purple LED $(30 \mathrm{~W})$. Then, the reaction mixture was concentrated. The residue was dissolved in $\mathrm{DCM}(20 \mathrm{~mL})$ and washed with saturated sodium carbonate aqueous solution $(20$ $\mathrm{mL})$. The aqueous phase was extracted with DCM $(3 \times 10 \mathrm{~mL})$. The organic phase was dried over $\mathrm{Na}_{2} \mathrm{SO}_{4}$ and the solvent was removed under reduced pressure. The residue was purified by chromatography on silica gel eluting with $\mathrm{CHCl}_{3} / \mathrm{MeOH}(50 / 1-30 / 1(\mathrm{v} / \mathrm{v}))$ to afford $143.4 \mathrm{mg}$ of 5 as a colorless oil $(69 \%$ yield, r.r. $=$ 10:1).

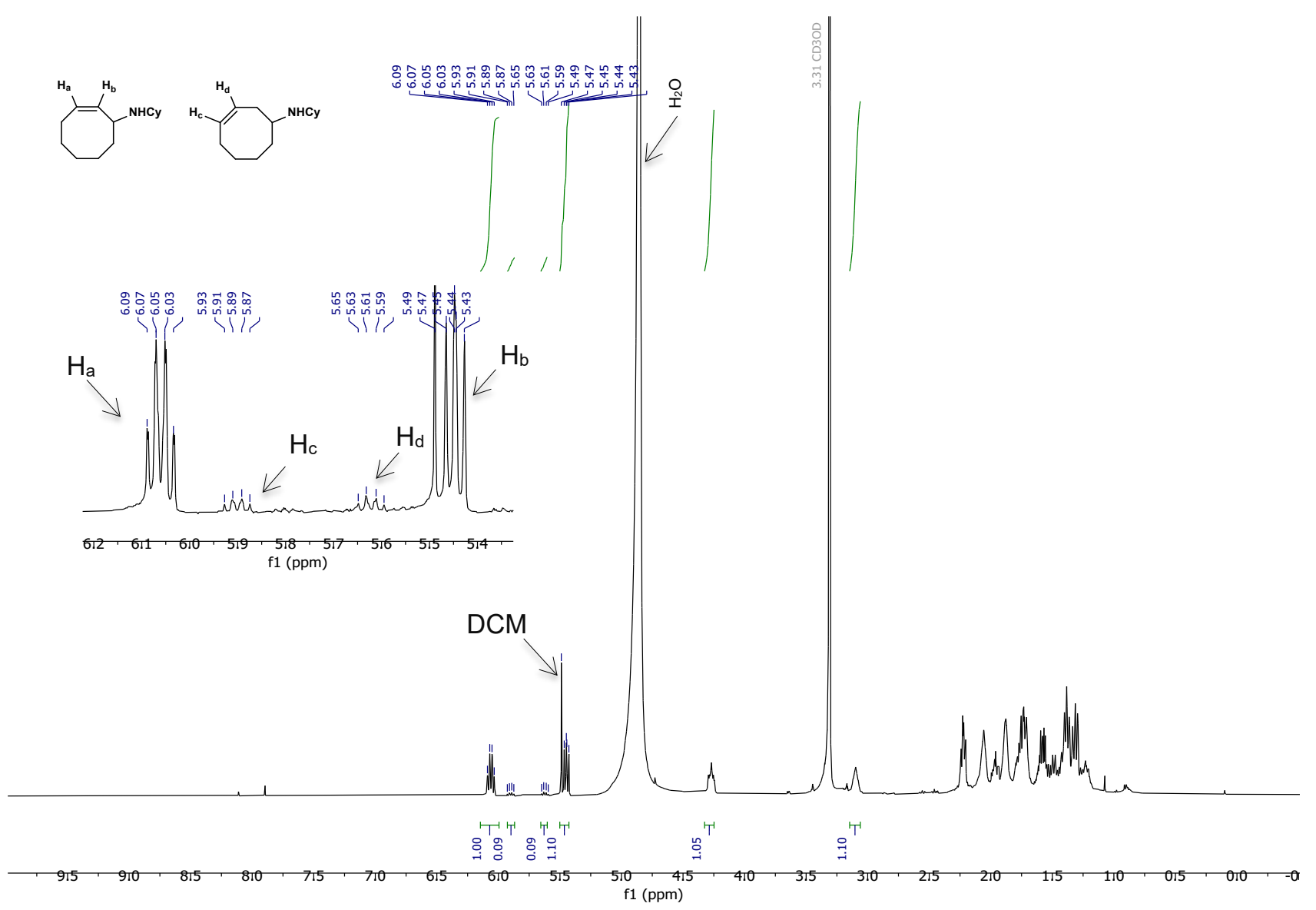

Figure S1. ${ }^{1} \mathrm{H}$ NMR of $1 \mathrm{in} \mathrm{MeOH}-d_{4}$. The ${ }^{1} \mathrm{H}$ NMR of $1 \mathrm{in} \mathrm{MeOH}-d_{4}$ shows a minor isomer: ${ }^{1} \mathrm{H}$ NMR $(500 \mathrm{MHz}$, $\left.\mathrm{MeOH}-d_{4}, 25^{\circ} \mathrm{C}, \delta\right): 5.90(\mathrm{dt}, J=9.5,8.5 \mathrm{~Hz}, 1 \mathrm{H}), 5.62(\mathrm{dt}, J=10.0,8.5 \mathrm{~Hz}, 1 \mathrm{H}$ ). The ratio is determined according to the integration ratio for $\mathrm{H}_{a}$ and $\mathrm{H}_{\mathrm{c}}$ as $10: 1$. 
(E)-N-(4-Phenyl-2-butenyl)cyclohexanamine 6

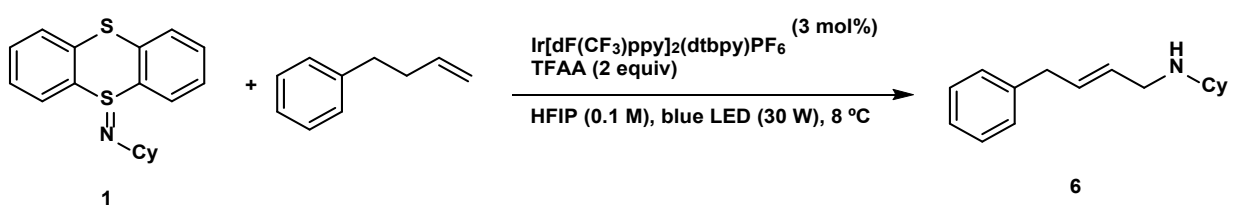

Under nitrogen atmosphere, to a 4-mL borosilicate vial equipped with a magnetic stir bar were added thianthrenylidene cyclohexylamine 1 (31.3 mg, $0.100 \mathrm{mmol}, 1.00$ equiv.), 4-phenyl-1-butene (23.0 $\mu \mathrm{L}, 19.8$ $\mathrm{mg}, 0.150 \mathrm{mmol}, 1.50$ equiv.), $\operatorname{Ir}\left[\mathrm{dF}\left(\mathrm{CF}_{3}\right) \mathrm{ppy}\right]_{2}(\mathrm{dtbpy}) \mathrm{PF}_{6}(3.3 \mathrm{mg}, 3.0 \mu \mathrm{mol}, 3.0 \mathrm{~mol} \%), \mathrm{HFIP}(1 \mathrm{~mL}, \mathrm{c}=0.1$ $\mathrm{M})$, and trifluoroacetic anhydride ( $28 \mu \mathrm{L}, 42 \mathrm{mg}, 0.20 \mathrm{mmol}, 2.0$ equiv.). The vial was sealed with a septumcap and irradiated for $3 \mathrm{~h}$ at $8{ }^{\circ} \mathrm{C}$ using a blue LED $(30 \mathrm{~W})$. Then, the reaction mixture was concentrated. The residue was dissolved in DCM $(5 \mathrm{~mL})$ and washed with saturated sodium carbonate aqueous solution $(5 \mathrm{~mL})$. The aqueous phase was extracted with DCM $(2 \times 5 \mathrm{~mL})$. The organic phase was dried over $\mathrm{Na}_{2} \mathrm{SO}_{4}$ and the solvent was removed under reduced pressure. The residue was purified by chromatography on silica gel eluting with $\mathrm{CHCl}_{3} / \mathrm{MeOH}(50 / 1-30 / 1(\mathrm{v} / \mathrm{v}))$ to afford $13.3 \mathrm{mg}$ of 6 as a colorless oil $(58 \%$ yield, $\mathrm{E}: \mathrm{Z}=7: 1)$.

$\mathbf{R}_{\boldsymbol{f}}=0.25\left(\mathrm{CHCl}_{3} / \mathrm{MeOH}=10 / 1(\mathrm{v} / \mathrm{v})\right)$.

\section{NMR Spectroscopy:}

${ }^{1} \mathrm{H}$ NMR $\left(500 \mathrm{MHz}, \mathrm{CDCl}_{3}, 25{ }^{\circ} \mathrm{C}, \delta\right): 7.28(\mathrm{t}, J=7.4 \mathrm{~Hz}, 2 \mathrm{H}), 7.23-7.18(\mathrm{~m}, 1 \mathrm{H}), 7.16(\mathrm{~d}, J=7.4 \mathrm{~Hz}$ $2 \mathrm{H}), 5.99$ (dt, $J=15.2,6.9 \mathrm{~Hz}, 1 \mathrm{H}), 5.85(\mathrm{dt}, J=15.4,7.0 \mathrm{~Hz}, 1 \mathrm{H}), 3.56(\mathrm{~d}, J=6.9 \mathrm{~Hz}, 2 \mathrm{H}), 3.42(\mathrm{~d}, J=$ $6.9 \mathrm{~Hz}, 2 \mathrm{H}), 2.93(\mathrm{ddd}, J=11.4,8.5,2.8 \mathrm{~Hz}, 1 \mathrm{H}), 2.20-2.09(\mathrm{~m}, 2 \mathrm{H}), 1.97-1.73(\mathrm{~m}, 2 \mathrm{H}), 1.66-1.51$ $(\mathrm{m}, 3 \mathrm{H}), 1.35-1.11(\mathrm{~m}, 3 \mathrm{H})$.

${ }^{13} \mathrm{C}$ NMR $\left(126 \mathrm{MHz}, \mathrm{CDCl}_{3}, 25^{\circ} \mathrm{C}, \delta\right): 139.2,138.4,128.7,128.6,126.5,121.6,55.4,45.9,38.9,29.4$, 25.0, 24.7.

HRMS-ESI(m/z) calc'd for $\mathrm{C}_{16} \mathrm{H}_{24} \mathrm{~N}[\mathrm{M}+1]^{+}, 230.1905$; found, 230.1903; deviation: -0.59 ppm.

(E)-2-(5-Cyclohexylamino-3-pentenyl)isoindoline-1,3-dione 7

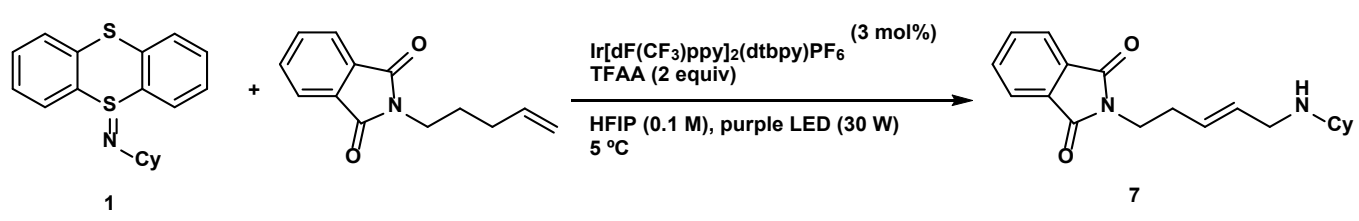

Under nitrogen atmosphere, to a 4-mL borosilicate vial equipped with a magnetic stir bar were added thianthrenylidene cyclohexylamine 1 (31.3 mg, $0.100 \mathrm{mmol}, 1.00$ equiv.), 2-(4-pentenyl)isoindoline-1,3-dione (32.2 mg, $0.150 \mathrm{mmol}, 1.50$ equiv.), Ir[dF(CF $)$ ppy $]_{2}(\mathrm{dtbpy}) \mathrm{PF}_{6}(3.3 \mathrm{mg}, 3.0 \mu \mathrm{mol}, 3.0 \mathrm{~mol} \%$ ), HFIP $(1 \mathrm{~mL}, \mathrm{c}=$ $0.1 \mathrm{M})$, and trifluoroacetic anhydride $(28 \mu \mathrm{L}, 42 \mathrm{mg}, 0.20 \mathrm{mmol}, 2.0$ equiv.). The vial was sealed with a septum-cap and irradiated for $3 \mathrm{~h}$ at $5{ }^{\circ} \mathrm{C}$ using a purple LED $(30 \mathrm{~W})$. Then, the reaction mixture was concentrated. The residue was dissolved in DCM $(5 \mathrm{~mL})$ and washed with saturated sodium carbonate aqueous solution $(5 \mathrm{~mL})$. The aqueous phase was extracted with DCM $(2 \times 5 \mathrm{~mL})$. The organic phase was 
dried over $\mathrm{Na}_{2} \mathrm{SO}_{4}$ and the solvent was removed under reduced pressure. The residue was purified by chromatography on silica gel eluting with $\mathrm{CHCl}_{3} / \mathrm{MeOH}(50 / 1-30 / 1(\mathrm{v} / \mathrm{v}))$ to afford $14.7 \mathrm{mg}$ of 7 as a colorless oil $(47 \%$ yield, $E: Z>20: 1)$.

$\mathbf{R}_{\boldsymbol{f}}=0.30\left(\mathrm{CHCl}_{3} / \mathrm{MeOH}=10 / 1(\mathrm{v} / \mathrm{v})\right)$.

\section{NMR Spectroscopy:}

${ }^{1} \mathrm{H}$ NMR $\left(500 \mathrm{MHz}, \mathrm{CDCl}_{3}, 25^{\circ} \mathrm{C}, \delta\right): 7.83(\mathrm{dd}, J=5.4,3.0 \mathrm{~Hz}, 2 \mathrm{H}), 7.71(\mathrm{dd}, J=5.5,3.0 \mathrm{~Hz}, 2 \mathrm{H}), 5.86$ (dt, $J=14.0,6.7 \mathrm{~Hz}, 1 \mathrm{H}), 5.77(\mathrm{dt}, J=15.0,6.8 \mathrm{~Hz}, 1 \mathrm{H}), 3.76(\mathrm{t}, J=6.8 \mathrm{~Hz}, 2 \mathrm{H}), 3.50(\mathrm{~d}, J=6.7 \mathrm{~Hz}$, $2 \mathrm{H}), 2.99-2.73(\mathrm{~m}, 1 \mathrm{H}), 2.59-2.34(\mathrm{~m}, 2 \mathrm{H}), 2.23-1.97(\mathrm{~m}, 2 \mathrm{H}), 1.91-1.74(\mathrm{~m}, 2 \mathrm{H}), 1.70-1.46(\mathrm{~m}$, $3 \mathrm{H}), 1.36-1.08(\mathrm{~m}, 3 \mathrm{H})$.

${ }^{13} \mathrm{C}$ NMR $\left(126 \mathrm{MHz}, \mathrm{CDCl}_{3}, 25{ }^{\circ} \mathrm{C}, \delta\right): 168.3,134.2,134.2,132.1,132.1,123.5,55.0,45.6,37.2,31.9$, 29.3, 25.0, 24.5.

HRMS-ESI(m/z) calc'd for $\mathrm{C}_{19} \mathrm{H}_{25} \mathrm{~N}_{2} \mathrm{O}_{2}[\mathrm{M}+1]^{+}, 313.1911$; found, 313.1911; deviation: $-0.12 \mathrm{ppm}$.

\section{(E)-4-Cyclohexylamino-2-butenyl benzoate 8}

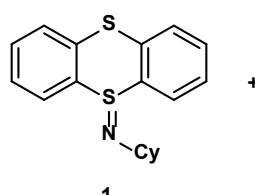

1

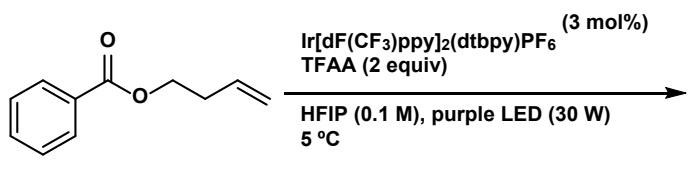

${ }^{\circ} \mathrm{C}$

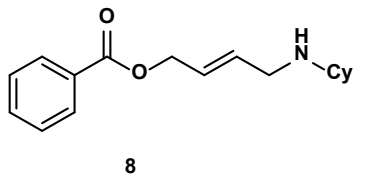

Under nitrogen atmosphere, to a 4-mL borosilicate vial equipped with a magnetic stir bar were added thianthrenylidene cyclohexylamine 1 (31.3 mg, $0.100 \mathrm{mmol}, 1.00$ equiv.), 3-butenyl benzoate (26.5 mg, 0.150 mmol, 1.50 equiv.), Ir[dF(CF $)$ ppy $]_{2}\left(\mathrm{dtbpy}_{\mathrm{P}} \mathrm{PF}_{6}(3.3 \mathrm{mg}, 3.0 \mu \mathrm{mol}, 3.0 \mathrm{~mol} \%), \mathrm{HFIP}(1 \mathrm{~mL}, \mathrm{c}=0.1 \mathrm{M})\right.$, and trifluoroacetic anhydride ( $28 \mu \mathrm{L}, 42 \mathrm{mg}, 0.20 \mathrm{mmol}, 2.0$ equiv.). The vial was sealed with a septum-cap and irradiated for $3 \mathrm{~h}$ at $5{ }^{\circ} \mathrm{C}$ using a purple LED $(30 \mathrm{~W})$. Then, the reaction mixture was concentrated. The residue was dissolved in DCM $(5 \mathrm{~mL})$ and washed with saturated sodium carbonate aqueous solution $(5 \mathrm{~mL})$. The aqueous phase was extracted with DCM $(2 \times 5 \mathrm{~mL})$. The organic phase was dried over $\mathrm{Na}_{2} \mathrm{SO}_{4}$ and the solvent was removed under reduced pressure. The residue was purified by chromatography on silica gel eluting with $\mathrm{CHCl}_{3} / \mathrm{MeOH}(50 / 1-30 / 1(\mathrm{v} / \mathrm{v}))$ to afford $11.0 \mathrm{mg}$ of 8 as a colorless oil (40\% yield, $\left.\mathrm{E}: Z=12: 1\right)$. $\mathbf{R}_{\boldsymbol{f}}=0.30\left(\mathrm{CHCl}_{3} / \mathrm{MeOH}=10 / 1(\mathrm{v} / \mathrm{v})\right)$.

\section{NMR Spectroscopy:}

${ }^{1} \mathrm{H}$ NMR $\left(500 \mathrm{MHz}, \mathrm{CDCl}_{3}, 25^{\circ} \mathrm{C}, \delta\right): 9.59(\mathrm{br}, 1 \mathrm{H}), 8.06(\mathrm{dd}, J=8.5,1.5 \mathrm{~Hz}, 2 \mathrm{H}), 7.60-7.53(\mathrm{~m}, 1 \mathrm{H})$, $7.44(\mathrm{t}, J=7.7 \mathrm{~Hz}, 2 \mathrm{H}), 6.18(\mathrm{dt}, J=13.7,6.5 \mathrm{~Hz}, 1 \mathrm{H}), 6.10(\mathrm{dt}, J=15.6,5.3 \mathrm{~Hz}, 1 \mathrm{H}), 4.82(\mathrm{~d}, J=5.0$ $\mathrm{Hz}, 2 \mathrm{H}), 3.96-3.52(\mathrm{~m}, 2 \mathrm{H}), 3.11-2.90(\mathrm{~m}, 1 \mathrm{H}), 2.33-2.07(\mathrm{~m}, 2 \mathrm{H}), 1.90-1.78(\mathrm{~m}, 2 \mathrm{H}), 1.71-1.56$ $(\mathrm{m}, 3 \mathrm{H}), 1.37-1.10(\mathrm{~m}, 3 \mathrm{H})$.

${ }^{13} \mathrm{C}$ NMR $\left(126 \mathrm{MHz} \mathrm{CDCl}_{3}, 25^{\circ} \mathrm{C}, \delta\right): 166.3,133.5,133.3,129.9,129.9,128.6,123.6,64.0,55.8,45.2$, 29.2, 24.9, 24.6 . 
HRMS-ESI(m/z) calc'd for $\mathrm{C}_{17} \mathrm{H}_{24} \mathrm{NO}_{2}[\mathrm{M}+1]^{+}, 274.1803$; found, 274.1802; deviation: $-0.46 \mathrm{ppm}$.

(E)-N-(2-Dodecenyl)cyclohexanamine 9

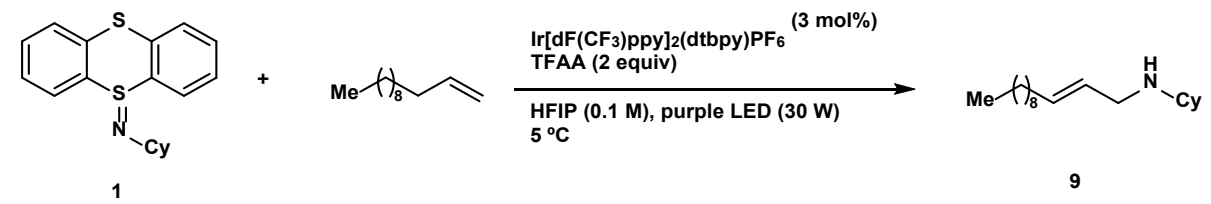

Under nitrogen atmosphere, to a 4-mL borosilicate vial equipped with a magnetic stir bar were added thianthrenylidene cyclohexylamine 1 (31.3 mg, $0.100 \mathrm{mmol}, 1.00$ equiv.), 1-dodecene (33.0 $\mu \mathrm{L}, 25.2 \mathrm{mg}$, $0.150 \mathrm{mmol}, 1.50$ equiv.), $\operatorname{Ir}\left[\mathrm{dF}\left(\mathrm{CF}_{3}\right) \mathrm{ppy}_{2}(\mathrm{dtbpy}) \mathrm{PF}_{6}(3.3 \mathrm{mg}, 3.0 \mu \mathrm{mol}, 3.0 \mathrm{~mol} \%), \mathrm{HFIP}(1 \mathrm{~mL}, \mathrm{c}=0.1 \mathrm{M})\right.$, and trifluoroacetic anhydride ( $28 \mu \mathrm{L}, 42 \mathrm{mg}, 0.20 \mathrm{mmol}, 2.0$ equiv.). The vial was sealed with a septum-cap and irradiated for $3 \mathrm{~h}$ at $5{ }^{\circ} \mathrm{C}$ using a purple LED $(30 \mathrm{~W})$. Then, the reaction mixture was concentrated. The residue was dissolved in $\mathrm{DCM}(5 \mathrm{~mL})$ and washed with saturated sodium carbonate aqueous solution $(5 \mathrm{~mL})$. The aqueous phase was extracted with DCM $(2 \times 5 \mathrm{~mL})$. The organic phase was dried over $\mathrm{Na}_{2} \mathrm{SO}_{4}$ and the solvent was removed under reduced pressure. The residue was purified by chromatography on silica gel eluting with $\mathrm{CHCl}_{3} / \mathrm{MeOH}(50 / 1-30 / 1(\mathrm{v} / \mathrm{v}))$ to afford $18.9 \mathrm{mg}$ of 9 as a colorless oil $(71 \%$ yield, $\mathrm{E}: \mathrm{Z}=8: 1)$.

$\mathbf{R}_{\boldsymbol{f}}=0.30\left(\mathrm{CHCl}_{3} / \mathrm{MeOH}=15 / 1(\mathrm{v} / \mathrm{v})\right)$.

\section{NMR Spectroscopy:}

${ }^{1} \mathrm{H}$ NMR (500 MHz, $\left.\mathrm{CDCl}_{3}, 25^{\circ} \mathrm{C}, \delta\right): 5.84(\mathrm{dt}, J=15.3,6.8 \mathrm{~Hz}, 1 \mathrm{H}), 5.68(\mathrm{dt}, J=15.0,7.0 \mathrm{~Hz}, 1 \mathrm{H}), 3.53$ $(\mathrm{d}, J=7.0 \mathrm{~Hz}, 2 \mathrm{H}), 2.99-2.89(\mathrm{~m}, 1 \mathrm{H}), 2.18-2.11(\mathrm{~m}, 2 \mathrm{H}), 2.09-2.02(\mathrm{~m}, 2 \mathrm{H}), 1.88-1.79(\mathrm{~m}, 2 \mathrm{H})$, $1.68-1.52(\mathrm{~m}, 3 \mathrm{H}), 1.31-1.21(\mathrm{~m}, 17 \mathrm{H}), 0.88(\mathrm{t}, J=6.9 \mathrm{~Hz}, 3 \mathrm{H})$.

${ }^{13} \mathrm{C}$ NMR $\left(126 \mathrm{MHz}, \mathrm{CDCl}_{3}, 25{ }^{\circ} \mathrm{C}, \delta\right): 140.7,119.9,55.0,45.9,32.5,32.0,29.8,29.7,29.6,29.5,29.4$, 29.2, 29.0, 24.7, 22.8, 14.3.

HRMS-ESI(m/z) calc'd for $\mathrm{C}_{18} \mathrm{H}_{36} \mathrm{~N}[\mathrm{M}+1]^{+}, 266.2843$; found, 266.2842; deviation: $-0.10 \mathrm{ppm}$.

(E)-N-(-2-Hexenyl)cyclohexanamine 10

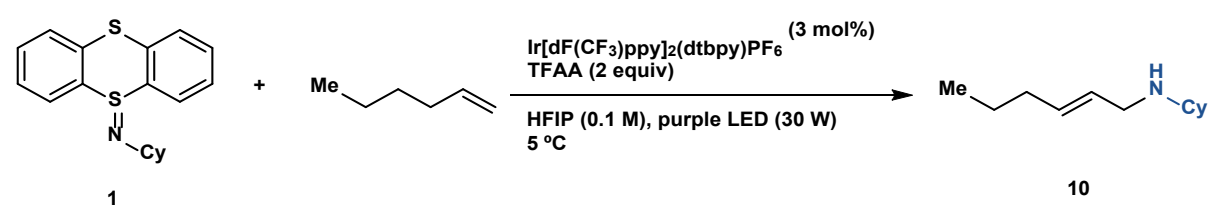

Under nitrogen atmosphere, to a 4-mL borosilicate vial equipped with a magnetic stir bar were added thianthrenylidene cyclohexylamine 1 (31.3 mg, $0.100 \mathrm{mmol}, 1.00$ equiv.), 1-hexene (19.0 $\mu \mathrm{L}, 12.6 \mathrm{mg}, 0.150$ mmol, 1.50 equiv.), $\operatorname{Ir}\left[\mathrm{dF}\left(\mathrm{CF}_{3}\right) \mathrm{ppy}\right]_{2}(\mathrm{dtbpy}) \mathrm{PF}_{6}(3.3 \mathrm{mg}, 3.0 \mu \mathrm{mol}, 3.0 \mathrm{~mol} \%$ ), HFIP (1 mL, c = $0.1 \mathrm{M})$, and trifluoroacetic anhydride ( $28 \mu \mathrm{L}, 42 \mathrm{mg}, 0.20 \mathrm{mmol}, 2.0$ equiv.). The vial was sealed with a septum-cap and irradiated for $3 \mathrm{~h}$ at $5{ }^{\circ} \mathrm{C}$ using a purple LED $(30 \mathrm{~W})$. Then, the reaction mixture was concentrated. The residue was dissolved in DCM $(5 \mathrm{~mL})$ and washed with saturated sodium carbonate aqueous solution $(5 \mathrm{~mL})$. The aqueous phase was extracted with DCM $(2 \times 5 \mathrm{~mL})$. The organic phase was dried over $\mathrm{Na}_{2} \mathrm{SO}_{4}$ and the 
solvent was removed under reduced pressure. The residue was purified by chromatography on silica gel eluting with $\mathrm{CHCl}_{3} / \mathrm{MeOH}(50 / 1-30 / 1(\mathrm{v} / \mathrm{v}))$ to afford $11.8 \mathrm{mg}$ of 10 as a colorless oil $(65 \%$ yield, $\mathrm{E}: \mathrm{Z}=6: 1)$.

$\boldsymbol{R}_{\boldsymbol{f}}=0.25\left(\mathrm{CHCl}_{3} / \mathrm{MeOH}=15 / 1(\mathrm{v} / \mathrm{v})\right)$.

\section{NMR Spectroscopy:}

${ }^{1} \mathrm{H}$ NMR $\left(500 \mathrm{MHz}, \mathrm{CDCl}_{3}, 25^{\circ} \mathrm{C}, \delta\right): 5.85(\mathrm{dt}, J=15.2,6.8 \mathrm{~Hz}, 1 \mathrm{H}), 5.71(\mathrm{dt}, J=15.3,7.0 \mathrm{~Hz}, 1 \mathrm{H}), 3.55$ $(\mathrm{d}, J=7.0 \mathrm{~Hz}, 2 \mathrm{H}), 3.00-2.84(\mathrm{~m}, 1 \mathrm{H}), 2.23-2.13(\mathrm{~m}, 2 \mathrm{H}), 2.10-2.02(\mathrm{~m}, 2 \mathrm{H}), 1.91-1.73(\mathrm{~m}, 2 \mathrm{H})$, $1.70-1.53(\mathrm{~m}, 3 \mathrm{H}), 1.41(\mathrm{q}, J=7.4 \mathrm{~Hz}, 2 \mathrm{H}), 1.29-1.14(\mathrm{~m}, 3 \mathrm{H}), 0.88(\mathrm{t}, J=7.4 \mathrm{~Hz}, 3 \mathrm{H})$.

${ }^{13} \mathrm{C}$ NMR (126 MHz, $\left.\mathrm{CDCl}_{3}, 25{ }^{\circ} \mathrm{C}, \delta\right): 140.5,120.0,55.1,45.9,34.5,29.2,25.0,24.7,22.1,13.8$.

HRMS-ESI(m/z) calc'd for $\mathrm{C}_{12} \mathrm{H}_{24} \mathrm{~N}$ [M+1] ${ }^{+}, 182.1901$; found, 182.1903; deviation: $1.28 \mathrm{ppm}$.

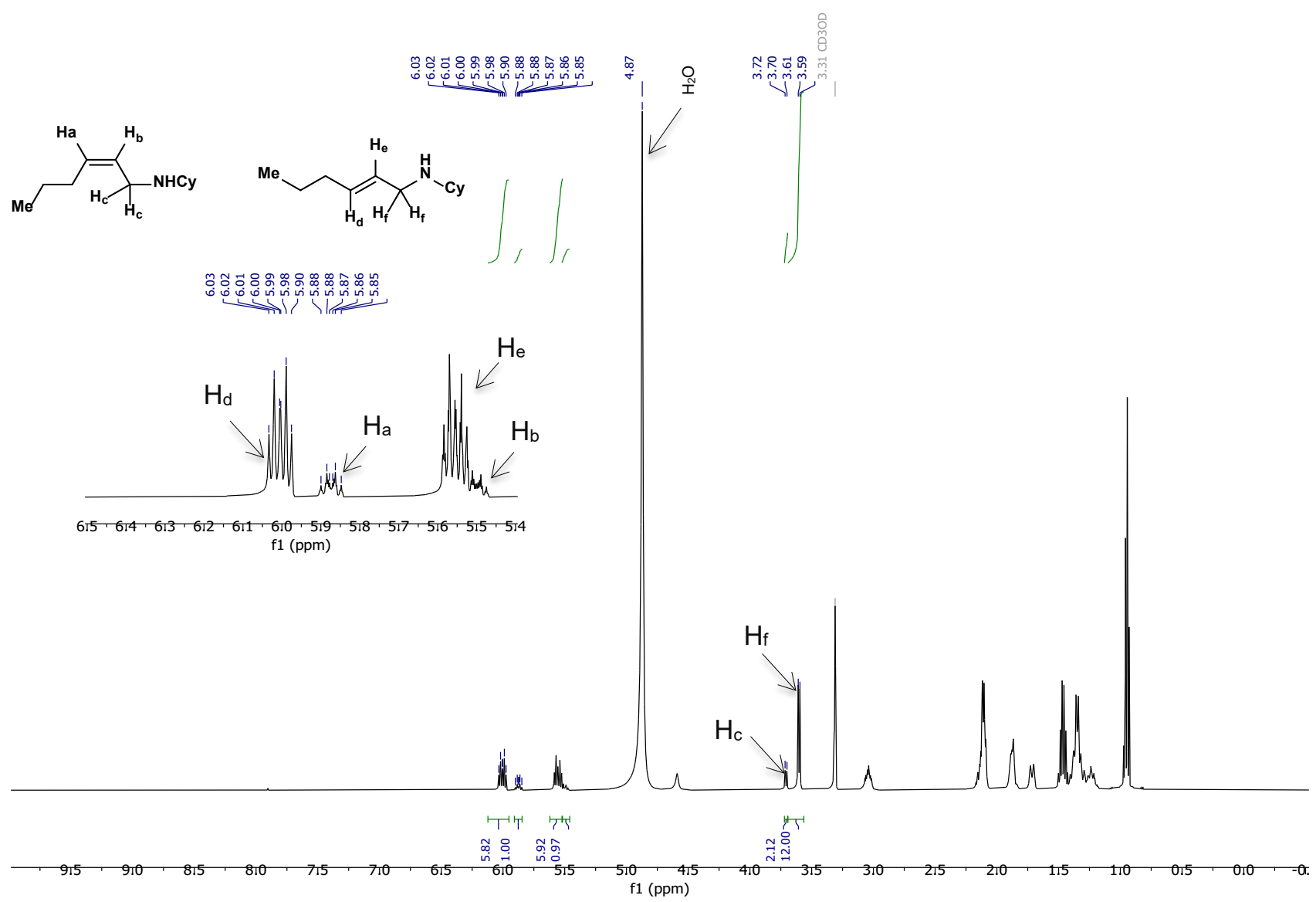

Figure S2. ${ }^{1} \mathrm{H}$ NMR of 10 in $\mathrm{MeOH}-d_{4}$. The ${ }^{1} \mathrm{H}$ NMR of 10 in $\mathrm{MeOH}-d_{4}$ clearly shows a cis-isomer: ${ }^{1} \mathrm{H}$ NMR $\left(500 \mathrm{MHz}, \mathrm{MeOH}-d_{4}, 25^{\circ} \mathrm{C}, \delta\right): 5.87(\mathrm{dt}, J=11.0,7.5 \mathrm{~Hz}, 1 \mathrm{H}), 5.50(\mathrm{dt}, J=11.0,7.0 \mathrm{~Hz}, 1 \mathrm{H}), 3.71(\mathrm{~d}, J=7.0$ $\mathrm{Hz}, 2 \mathrm{H})$. The $\mathrm{E}: \mathrm{Z}$ ratio is determined according to the integration ratio for $\mathrm{H}_{f}$ and $\mathrm{H}_{c}$ as $6: 1$. 
(E)-N-(4-Methyl-2-pentenyl)cyclohexanamine 11

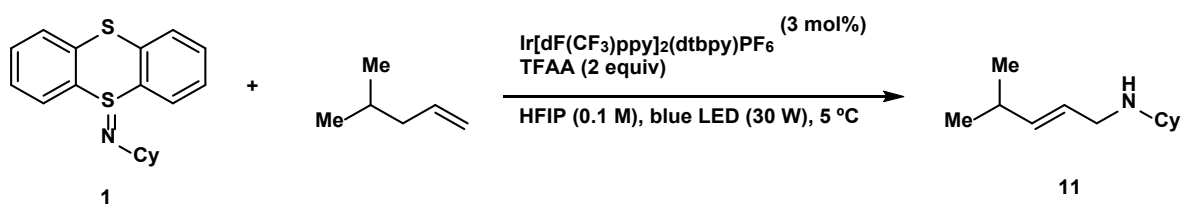

Under nitrogen atmosphere, to a 4-mL borosilicate vial equipped with a magnetic stir bar were added thianthrenylidene cyclohexylamine $1(31.3 \mathrm{mg}, 0.100 \mathrm{mmol}, 1.00$ equiv.), 4-methyl-1-pentene (19.0 $\mu \mathrm{L}, 12.6$ $\mathrm{mg}, 0.150 \mathrm{mmol}, 1.50$ equiv.), $\operatorname{Ir}\left[\mathrm{dF}\left(\mathrm{CF}_{3}\right) \mathrm{ppy}\right]_{2}(\mathrm{dtbpy}) \mathrm{PF}_{6}(3.3 \mathrm{mg}, 3.0 \mu \mathrm{mol}, 3.0 \mathrm{~mol} \%), \mathrm{HFIP}(1 \mathrm{~mL}, \mathrm{c}=0.1$ $\mathrm{M})$, and trifluoroacetic anhydride ( $28 \mu \mathrm{L}, 42 \mathrm{mg}, 0.20 \mathrm{mmol}, 2.0$ equiv.). The vial was sealed with a septumcap and irradiated for $3 \mathrm{~h}$ at $8{ }^{\circ} \mathrm{C}$ using a blue LED $(30 \mathrm{~W})$. Then, the reaction mixture was concentrated. The residue was dissolved in DCM $(5 \mathrm{~mL})$ and washed with saturated sodium carbonate aqueous solution $(5 \mathrm{~mL})$. The aqueous phase was extracted with DCM $(2 \times 5 \mathrm{~mL})$. The organic phase was dried over $\mathrm{Na}_{2} \mathrm{SO}_{4}$ and the solvent was removed under reduced pressure. The residue was purified by chromatography on silica gel eluting with $\mathrm{CHCl}_{3} / \mathrm{MeOH}(50 / 1-30 / 1(\mathrm{v} / \mathrm{v}))$ to afford $12.9 \mathrm{mg}$ of 11 as a colorless oil $(71 \%$ yield, $\mathrm{E}: Z=16: 1)$.

$\mathbf{R}_{\boldsymbol{f}}=0.25\left(\mathrm{CHCl}_{3} / \mathrm{MeOH}=15 / 1(\mathrm{v} / \mathrm{v})\right)$.

\section{NMR Spectroscopy:}

${ }^{1} \mathrm{H}$ NMR $\left(500 \mathrm{MHz}, \mathrm{CDCl}_{3}, 25^{\circ} \mathrm{C}, \delta\right): 5.81(\mathrm{dd}, J=15.4,6.8 \mathrm{~Hz}, 1 \mathrm{H}), 5.63(\mathrm{dtd}, J=15.5,7.0,1.5 \mathrm{~Hz}$, $1 \mathrm{H}), 3.54(\mathrm{~d}, J=7.0 \mathrm{~Hz}, 2 \mathrm{H}), 3.04-2.80(\mathrm{~m}, 1 \mathrm{H}), 2.43-2.26(\mathrm{~m}, 1 \mathrm{H}), 2.21-2.09(\mathrm{~m}, 2 \mathrm{H}), 1.92-1.75$ $(\mathrm{m}, 2 \mathrm{H}), 1.70-1.52(\mathrm{~m}, 3 \mathrm{H}), 1.32-1.18(\mathrm{~m}, 3 \mathrm{H}), 1.00(\mathrm{~d}, J=6.7 \mathrm{~Hz}, 6 \mathrm{H})$.

${ }^{13} \mathrm{C}$ NMR $\left(126 \mathrm{MHz}, \mathrm{CDCl}_{3}, 25^{\circ} \mathrm{C}, \delta\right): 147.2,117.0,55.4,46.2,31.2,29.2,25.0,24.6,22.0$.

HRMS-ESI(m/z) calc'd for $\mathrm{C}_{12} \mathrm{H}_{24} \mathrm{~N}[\mathrm{M}+1]^{+}, 182.1903$; found, 182.1903; deviation: $0.24 \mathrm{ppm}$.

(E)-N-(5-(tert-Butyldiphenylsilyl)oxy-2-pentenyl)cyclohexanamine 12

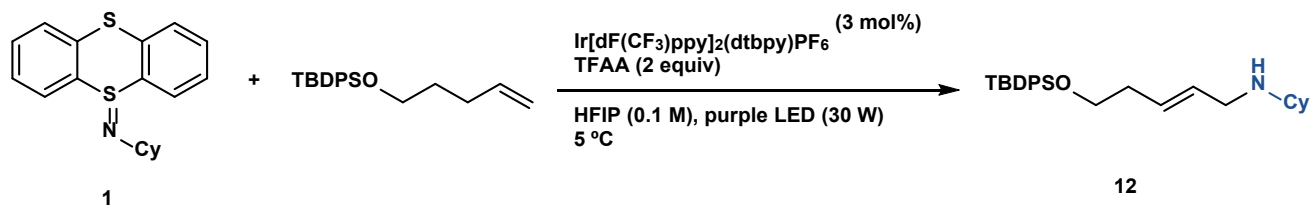

Under nitrogen atmosphere, to a 4-mL borosilicate vial equipped with a magnetic stir bar were added thianthrenylidene cyclohexylamine 1 (31.3 mg, $0.100 \mathrm{mmol}, 1.00$ equiv.), tert-butyl(-4pentenyloxy)diphenylsilane (48.6 mg, $0.150 \mathrm{mmol}, 1.50$ equiv.), $\operatorname{Ir}\left[\mathrm{dF}\left(\mathrm{CF}_{3}\right) \text { ppy }\right]_{2}$ (dtbpy)PF6 (3.3 mg, $3.0 \mu \mathrm{mol}$, $3.0 \mathrm{~mol} \%)$, HFIP ( $1 \mathrm{~mL}, \mathrm{c}=0.1 \mathrm{M})$, and trifluoroacetic anhydride $(28 \mu \mathrm{L}, 42 \mathrm{mg}, 0.20 \mathrm{mmol}, 2.0$ equiv.). The vial was sealed with a septum-cap and irradiated for $3 \mathrm{~h}$ at $5{ }^{\circ} \mathrm{C}$ using a purple LED $(30 \mathrm{~W})$. Then, the reaction mixture was concentrated. The residue was dissolved in DCM $(5 \mathrm{~mL})$ and washed with saturated sodium carbonate aqueous solution $(5 \mathrm{~mL})$. The aqueous phase was extracted with $\mathrm{DCM}(2 \times 5 \mathrm{~mL})$. The organic phase was dried over $\mathrm{Na}_{2} \mathrm{SO}_{4}$ and the solvent was removed under reduced pressure. The residue was purified by chromatography on silica gel eluting with $\mathrm{CHCl}_{3} / \mathrm{MeOH}(50 / 1-30 / 1(\mathrm{v} / \mathrm{v}))$ to afford $21.6 \mathrm{mg}$ of 
12 as a colorless oil $(51 \%$ yield, $E: Z=5: 1)$.

$\boldsymbol{R} \boldsymbol{f}=0.30\left(\mathrm{CHCl}_{3} / \mathrm{MeOH}=10 / 1(\mathrm{v} / \mathrm{v})\right)$.

\section{NMR Spectroscopy:}

${ }^{1} \mathrm{H}$ NMR $\left(600 \mathrm{MHz}, \mathrm{CDCl}_{3}, 25^{\circ} \mathrm{C}, \delta\right): 7.64(\mathrm{dd}, J=8.0,1.5 \mathrm{~Hz}, 4 \mathrm{H}), 7.46-7.39(\mathrm{~m}, 2 \mathrm{H}), 7.41-7.35(\mathrm{~m}$, $4 \mathrm{H}), 5.85(\mathrm{dt}, J=15.3,6.8 \mathrm{~Hz}, 1 \mathrm{H}), 5.75(\mathrm{dt}, J=15.3,7.0 \mathrm{~Hz}, 1 \mathrm{H}), 3.69(\mathrm{t}, J=6.4 \mathrm{~Hz}, 2 \mathrm{H}), 3.51(\mathrm{~d}, J=$ $6.9 \mathrm{~Hz}, 2 \mathrm{H}), 2.98-2.77(\mathrm{~m}, 1 \mathrm{H}), 2.42-2.28(\mathrm{~m}, 2 \mathrm{H}), 2.19-2.03(\mathrm{~m}, 2 \mathrm{H}), 1.84-1.75(\mathrm{~m}, 2 \mathrm{H}), 1.68-$ $1.49(\mathrm{~m}, 3 \mathrm{H}), 1.24-1.13(\mathrm{~m}, 3 \mathrm{H}), 1.03(\mathrm{~s}, 9 \mathrm{H})$.

${ }^{13} \mathrm{C} \mathrm{NMR}\left(151 \mathrm{MHz}, \mathrm{CDCl}_{3}, 2{ }^{\circ} \mathrm{C}, \delta\right): 135.7,133.9,129.9,129.8,127.9,127.8,63.2,55.0,35.9,29.5$, 27.0, 24.6, 19.4 .

HRMS-ESI(m/z) calc'd for $\mathrm{C}_{27} \mathrm{H}_{40} \mathrm{NOSi}[\mathrm{M}+1]^{+}$, 422.2878; found, 422.2874; deviation: -1.05 ppm.

(E)-N-(4-Bromo-2-butenyl)cyclohexanaminium trifluoroacetate 13

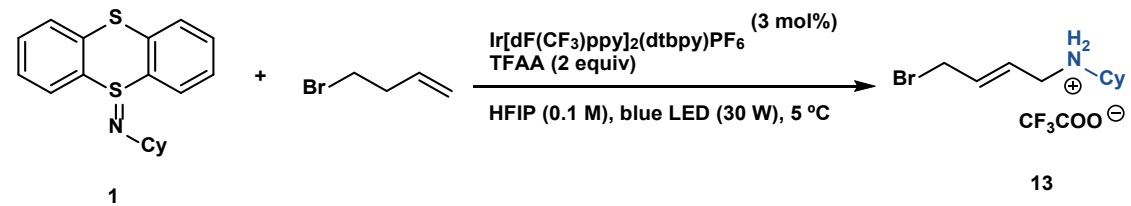

Under nitrogen atmosphere, to a 4-mL borosilicate vial equipped with a magnetic stir bar were added thianthrenylidene cyclohexylamine 1 (31.3 mg, $0.100 \mathrm{mmol}, 1.00$ equiv.), 4-bromo-1-butene (15.0 $\mu \mathrm{L}, 20.3$ mg, 0.150 mmol, 1.50 equiv.), Ir[dF(CF $)$ ppy $]_{2}(\mathrm{dtbpy}) \mathrm{PF}_{6}(3.3 \mathrm{mg}, 3.0 \mu \mathrm{mol}, 3.0 \mathrm{~mol} \%), \mathrm{HFIP}(1 \mathrm{~mL}, \mathrm{c}=0.1$ $\mathrm{M})$, and trifluoroacetic anhydride ( $28 \mu \mathrm{L}, 42 \mathrm{mg}, 0.20 \mathrm{mmol}, 2.0$ equiv.). The vial was sealed with a septumcap and irradiated for $3 \mathrm{~h}$ at $8{ }^{\circ} \mathrm{C}$ using a blue LED (30 W). Then, the reaction mixture was concentrated. The residue was purified by chromatography on silica gel eluting with $\mathrm{CHCl}_{3} / \mathrm{MeOH}(50 / 1-30 / 1(\mathrm{v} / \mathrm{v}))$ to afford $15.7 \mathrm{mg}$ of 13 as a colorless oil (45\% yield, $E: Z=10: 1)$.

$\mathbf{R}_{\boldsymbol{f}}=0.25\left(\mathrm{CHCl}_{3} / \mathrm{MeOH}=10 / 1(\mathrm{v} / \mathrm{v})\right)$.

\section{NMR Spectroscopy:}

${ }^{1} \mathrm{H}$ NMR (500 MHz, $\left.\mathrm{CDCl}_{3}, 25^{\circ} \mathrm{C}, \delta\right): 9.48(\mathrm{br}, 2 \mathrm{H}), 6.32-5.93(\mathrm{~m}, 2 \mathrm{H}), 4.07(\mathrm{dd}, J=6.4,1.0 \mathrm{~Hz}, 2 \mathrm{H}$ ), $3.75-3.52(\mathrm{~m}, 2 \mathrm{H}), 3.07-2.85(\mathrm{~m}, 1 \mathrm{H}), 2.27-2.05(\mathrm{~m}, 2 \mathrm{H}), 1.93-1.78(\mathrm{~m}, 2 \mathrm{H}), 1.77-1.60(\mathrm{~m}, 3 \mathrm{H})$, $1.38-1.13(\mathrm{~m}, 3 \mathrm{H})$.

${ }^{13} \mathrm{C}$ NMR (151 MHz, $\left.\mathrm{CDCl}_{3}, 25^{\circ} \mathrm{C}, \delta\right): 134.9,124.0,55.9,44.9,43.4,29.2,24.9,24.6$.

${ }^{19}$ F NMR (471 MHz, $\left.\mathrm{CDCl}_{3}, 298 \mathrm{~K}, \delta\right):-75.71$.

HRMS-ESI(m/z) calc'd for $\mathrm{C}_{10} \mathrm{H}_{19} \mathrm{NBr}\left[\mathrm{M}^{+}, 232.0698\right.$; found, 232.0695; deviation: $-0.87 \mathrm{ppm}$. 
(E)-6-(Cyclohexylamino)-4-hexen-1-ol 14
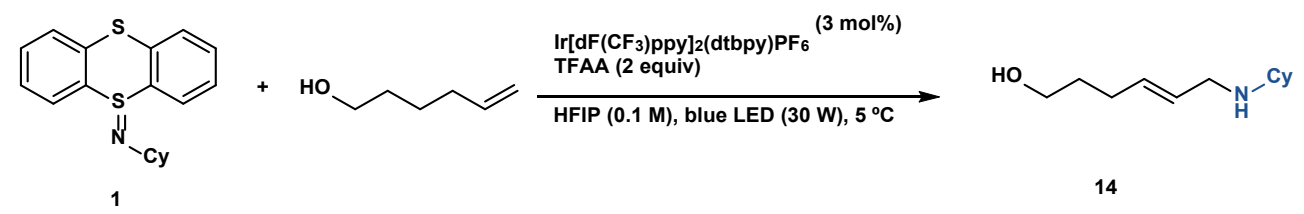

Under nitrogen atmosphere, to a 4-mL borosilicate vial equipped with a magnetic stir bar were added thianthrenylidene cyclohexylamine 1 (31.3 mg, $0.100 \mathrm{mmol}, 1.00$ equiv.), 5-hexen-1-ol (18.0 $\mu \mathrm{L}, 15.0 \mathrm{mg}$, $0.150 \mathrm{mmol}, 1.50$ equiv.), $\operatorname{Ir}\left[\mathrm{dF}\left(\mathrm{CF}_{3}\right) \mathrm{ppy}_{2}(\mathrm{dtbpy}) \mathrm{PF}_{6}(3.3 \mathrm{mg}, 3.0 \mu \mathrm{mol}, 3.0 \mathrm{~mol} \%), \mathrm{HFIP}(1 \mathrm{~mL}, \mathrm{c}=0.1 \mathrm{M})\right.$, and trifluoroacetic anhydride ( $28 \mu \mathrm{L}, 42 \mathrm{mg}, 0.20 \mathrm{mmol}, 2.0$ equiv.). The vial was sealed with a septum-cap and irradiated for $3 \mathrm{~h}$ at $8{ }^{\circ} \mathrm{C}$ using a blue LED $(30 \mathrm{~W})$. Then, the reaction mixture was concentrated. The residue was dissolved in $\mathrm{DCM}(5 \mathrm{~mL})$ and washed with saturated sodium carbonate aqueous solution $(5 \mathrm{~mL})$. The aqueous phase was extracted with DCM $(2 \times 5 \mathrm{~mL})$. The organic phase was dried over $\mathrm{Na}_{2} \mathrm{SO}_{4}$ and the solvent was removed under reduced pressure. The residue was purified by chromatography on silica gel eluting with $\mathrm{CHCl}_{3} / \mathrm{MeOH}(50 / 1-20 / 1(\mathrm{v} / \mathrm{v}))$ to afford $11.0 \mathrm{mg}$ of 14 as a colorless oil (56\% yield, $\left.\mathrm{E}: \mathrm{Z}=12: 1\right)$.

$\boldsymbol{R}_{\boldsymbol{f}}=0.15\left(\mathrm{CHCl}_{3} / \mathrm{MeOH}=10 / 1(\mathrm{v} / \mathrm{v})\right)$.

\section{NMR Spectroscopy:}

${ }^{1} \mathrm{H}$ NMR $\left(600 \mathrm{MHz}, \mathrm{CDCl}_{3}, 25^{\circ} \mathrm{C}, \delta\right): 5.68-5.51(\mathrm{~m}, 2 \mathrm{H}), 3.65(\mathrm{t}, \mathrm{J}=6.5 \mathrm{~Hz}, 2 \mathrm{H}), 3.24(\mathrm{~d}, J=5.4 \mathrm{~Hz}$, 2H), $2.48(\mathrm{tt}, J=10.6,3.7 \mathrm{~Hz}, 1 \mathrm{H}), 2.18-2.07(\mathrm{~m}, 2 \mathrm{H}), 1.94-1.85(\mathrm{~m}, 2 \mathrm{H}), 1.77-1.70(\mathrm{~m}, 2 \mathrm{H}), 1.68-$ $1.61(\mathrm{~m}, 2 \mathrm{H}), 1.30-1.22(\mathrm{~m}, 4 \mathrm{H}), 1.17-1.08(\mathrm{~m}, 2 \mathrm{H})$.

${ }^{13} \mathrm{C}$ NMR $\left(151 \mathrm{MHz}, \mathrm{CDCl}_{3}, 25{ }^{\circ} \mathrm{C}, \delta\right): 132.4,128.6,62.5,56.3,48.6,33.3,32.2,28.9,26.2,25.2$.

HRMS-ESI(m/z) calc'd for $\mathrm{C}_{12} \mathrm{H}_{24} \mathrm{NO}[\mathrm{M}+1]^{+}, 198.1854$; found, 198.1852; deviation: $-0.81 \mathrm{ppm}$.

(E)-7-Cyclohexylamino-5-heptenoic acid 15

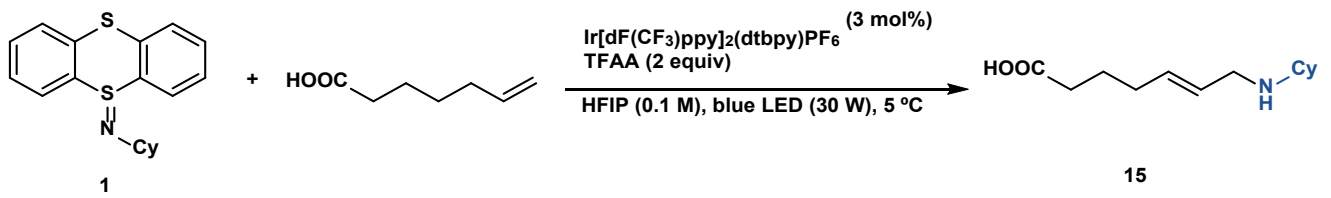

Under nitrogen atmosphere, to a 4-mL borosilicate vial equipped with a magnetic stir bar were added thianthrenylidene cyclohexylamine 1 (31.3 mg, $0.100 \mathrm{mmol}, 1.00$ equiv.), 6-heptenoic acid (20.0 $\mu \mathrm{L}, 19.2 \mathrm{mg}$, $0.150 \mathrm{mmol}, 1.50$ equiv.), $\operatorname{Ir}\left[\mathrm{dF}\left(\mathrm{CF}_{3}\right) \mathrm{ppy}_{2}(\mathrm{dtbpy}) \mathrm{PF}_{6}(3.3 \mathrm{mg}, 3.0 \mu \mathrm{mol}, 3.0 \mathrm{~mol} \%), \mathrm{HFIP}(1 \mathrm{~mL}, \mathrm{c}=0.1 \mathrm{M})\right.$, and trifluoroacetic anhydride ( $28 \mu \mathrm{L}, 42 \mathrm{mg}, 0.20 \mathrm{mmol}, 2.0$ equiv.). The vial was sealed with a septum-cap and irradiated for $3 \mathrm{~h}$ at $8{ }^{\circ} \mathrm{C}$ using a blue LED $(30 \mathrm{~W})$. Then, the reaction mixture was concentrated. The residue was dissolved in DCM $(5 \mathrm{~mL})$ and washed with saturated sodium carbonate aqueous solution $(5 \mathrm{~mL})$. The aqueous phase was extracted with DCM $(2 \times 5 \mathrm{~mL})$. The organic phase was dried over $\mathrm{Na}_{2} \mathrm{SO}_{4}$ and the solvent was removed under reduced pressure. The residue was purified by chromatography on silica gel eluting with $\mathrm{CHCl}_{3} / \mathrm{MeOH}(50 / 1-10 / 1(\mathrm{v} / \mathrm{v}))$ to afford $14.7 \mathrm{mg}$ of 15 as a colorless solid $(65 \%$ yield, $\mathrm{E}: \mathrm{Z}=9: 1)$. 
$\mathbf{R}_{\boldsymbol{f}}=0.10\left(\mathrm{CHCl}_{3} / \mathrm{MeOH}=10 / 1(\mathrm{v} / \mathrm{v})\right)$.

\section{NMR Spectroscopy:}

${ }^{1} \mathrm{H}$ NMR $\left(500 \mathrm{MHz}, \mathrm{CDCl}_{3}, 25^{\circ} \mathrm{C}, \delta\right): 5.85(\mathrm{dt}, J=15.4,6.7 \mathrm{~Hz}, 1 \mathrm{H}), 5.81-5.71(\mathrm{~m}, 1 \mathrm{H}), 3.56(\mathrm{~d}, J=6.8$ $\mathrm{Hz}, 2 \mathrm{H}), 3.05-2.84(\mathrm{~m}, 1 \mathrm{H}), 2.52(\mathrm{t}, J=7.5 \mathrm{~Hz}, 2 \mathrm{H}), 2.24-2.05(\mathrm{~m}, 4 \mathrm{H}), 1.92-1.76(\mathrm{~m}, 4 \mathrm{H}), 1.70-$ $1.53(\mathrm{~m}, 3 \mathrm{H}), 1.35-1.10(\mathrm{~m}, 3 \mathrm{H})$.

${ }^{13} \mathrm{C}$ NMR $\left(126 \mathrm{MHz}, \mathrm{CDCl}_{3}, 25{ }^{\circ} \mathrm{C}, \delta\right): 169.9,138.5,121.3,55.1,45.4,32.5,31.3,29.0,24.8,24.5,23.6$. HRMS-ESI(m/z) calc'd for $\mathrm{C}_{13} \mathrm{H}_{24} \mathrm{NO}_{2}[\mathrm{M}+1]^{+}, 226.1802$; found, 226.1802; deviation: -0.38 ppm.

(E)-N-(2-Octen-7-ynyl)cyclohexanamine 16

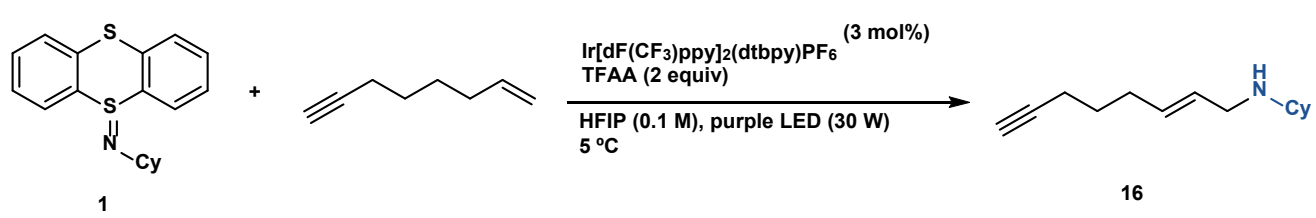

Under nitrogen atmosphere, to a 4-mL borosilicate vial equipped with a magnetic stir bar were added thianthrenylidene cyclohexylamine 1 (31.3 mg, $0.100 \mathrm{mmol}, 1.00$ equiv.), 1-octen-7-yne (16.2 mg, 0.150 mmol, 1.50 equiv.), Ir[dF(CF3)ppy $]_{2}(\mathrm{dtbpy}) \mathrm{PF}_{6}(3.3 \mathrm{mg}, 3.0 \mu \mathrm{mol}, 3.0 \mathrm{~mol} \%), \mathrm{HFIP}(1 \mathrm{~mL}, \mathrm{c}=0.1 \mathrm{M})$, and trifluoroacetic anhydride ( $28 \mu \mathrm{L}, 42 \mathrm{mg}, 0.20 \mathrm{mmol}, 2.0$ equiv.). The vial was sealed with a septum-cap and irradiated for $3 \mathrm{~h}$ at $5{ }^{\circ} \mathrm{C}$ using a purple LED $(30 \mathrm{~W})$. Then, the reaction mixture was concentrated. The residue was dissolved in $\mathrm{DCM}(5 \mathrm{~mL})$ and washed with saturated sodium carbonate aqueous solution $(5 \mathrm{~mL})$. The aqueous phase was extracted with DCM $(2 \times 5 \mathrm{~mL})$. The organic phase was dried over $\mathrm{Na}_{2} \mathrm{SO}_{4}$ and the solvent was removed under reduced pressure. The residue was purified by chromatography on silica gel eluting with $\mathrm{CHCl}_{3} / \mathrm{MeOH}(50 / 1-30 / 1(\mathrm{v} / \mathrm{v}))$ to afford $9.4 \mathrm{mg}$ of 16 as a colorless solid (45\% yield, $\left.\mathrm{E}: \mathrm{Z}=7: 1\right)$.

$\boldsymbol{R}_{\boldsymbol{f}}=0.25\left(\mathrm{CHCl}_{3} / \mathrm{MeOH}=10 / 1(\mathrm{v} / \mathrm{v})\right)$.

\section{NMR Spectroscopy:}

${ }^{1} \mathrm{H}$ NMR $\left(600 \mathrm{MHz}, \mathrm{CDCl}_{3}, 25^{\circ} \mathrm{C}, \delta\right): 5.85(\mathrm{dt}, J=15.4,6.7 \mathrm{~Hz}, 1 \mathrm{H}), 5.78-5.71(\mathrm{~m}, 1 \mathrm{H}), 3.54(\mathrm{~d}, J=6.9$ $\mathrm{Hz}, 2 \mathrm{H}), 3.01-2.83(\mathrm{~m}, 1 \mathrm{H}), 2.28-2.11(\mathrm{~m}, 5 \mathrm{H}), 1.95(\mathrm{t}, J=2.6 \mathrm{~Hz}, 1 \mathrm{H}), 1.87-1.79(\mathrm{~m}, 2 \mathrm{H}), 1.72-$ $1.53(\mathrm{~m}, 5 \mathrm{H}), 1.33-1.16(\mathrm{~m}, 4 \mathrm{H})$.

${ }^{13} \mathrm{C}$ NMR $\left(151 \mathrm{MHz}, \mathrm{CDCl}_{3}, 25^{\circ} \mathrm{C}, \delta\right): 139.1,121.1,83.9,69.0,55.3,45.9,31.4,29.3,27.6,25.0,24.7$, 18.0.

HRMS-ESI(m/z) calc'd for $\mathrm{C}_{14} \mathrm{H}_{24} \mathrm{~N}[\mathrm{M}+1]^{+}, 206.1901$; found, 206.1903; deviation: 0.89 ppm.

(E)-N-(2,5-Hexadienyl)cyclohexanamine 17

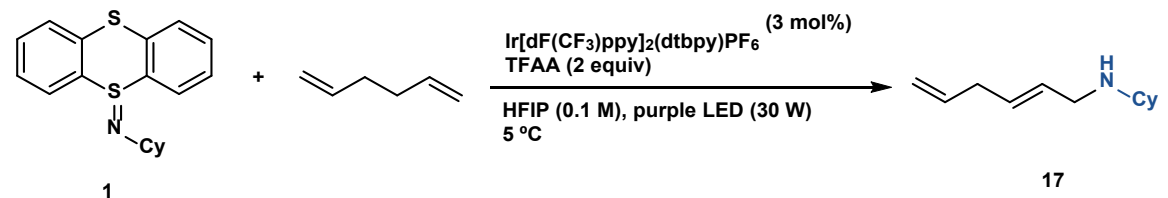


Under nitrogen atmosphere, to a 4-mL borosilicate vial equipped with a magnetic stir bar were added thianthrenylidene cyclohexylamine 1 (31.3 mg, $0.100 \mathrm{mmol}, 1.00$ equiv.), 1,5-hexadiene (18.0 $\mu \mathrm{L}, 12.3 \mathrm{mg}$, 0.150 mmol, 1.50 equiv.), $\operatorname{Ir}\left[\mathrm{dF}\left(\mathrm{CF}_{3}\right) \mathrm{ppy}_{2}(\mathrm{dtbpy}) \mathrm{PF}_{6}(3.3 \mathrm{mg}, 3.0 \mu \mathrm{mol}, 3.0 \mathrm{~mol} \%), \mathrm{HFIP}(1 \mathrm{~mL}, \mathrm{c}=0.1 \mathrm{M})\right.$, and trifluoroacetic anhydride ( $28 \mu \mathrm{L}, 42 \mathrm{mg}, 0.20 \mathrm{mmol}, 2.0$ equiv.). The vial was sealed with a septum-cap and irradiated for $3 \mathrm{~h}$ at $5{ }^{\circ} \mathrm{C}$ using a purple LED $(30 \mathrm{~W})$. Then, the reaction mixture was concentrated. The residue was dissolved in DCM $(5 \mathrm{~mL})$ and washed with saturated sodium carbonate aqueous solution $(5 \mathrm{~mL})$. The aqueous phase was extracted with DCM $(2 \times 5 \mathrm{~mL})$. The organic phase was dried over $\mathrm{Na}_{2} \mathrm{SO}_{4}$ and the solvent was removed under reduced pressure. The residue was purified by chromatography on silica gel eluting with $\mathrm{CHCl}_{3} / \mathrm{MeOH}(50 / 1-30 / 1(\mathrm{v} / \mathrm{v}))$ to afford $13.0 \mathrm{mg}$ of 17 as a colorless solid $(72 \%$ yield, $\mathrm{E}: \mathrm{Z}=\mathbf{8 : 1})$.

$\mathbf{R}_{\boldsymbol{f}}=0.25\left(\mathrm{CHCl}_{3} / \mathrm{MeOH}=10 / 1(\mathrm{v} / \mathrm{v})\right)$.

\section{NMR Spectroscopy:}

${ }^{1} \mathrm{H}$ NMR $\left(500 \mathrm{MHz}, \mathrm{CDCl}_{3}, 2{ }^{\circ} \mathrm{C}, \delta\right): 5.89(\mathrm{dt}, J=15.5,6.6 \mathrm{~Hz}, 1 \mathrm{H}), 5.84-5.74(\mathrm{~m}, 2 \mathrm{H}), 5.07-5.01$ (m, $2 \mathrm{H}), 3.57(\mathrm{~d}, J=7.0 \mathrm{~Hz}, 2 \mathrm{H}), 3.04-2.90(\mathrm{~m}, 1 \mathrm{H}), 2.90-2.80(\mathrm{~m}, 2 \mathrm{H}), 2.17(\mathrm{~d}, J=12.4 \mathrm{~Hz}, 2 \mathrm{H}), 1.93-$ $1.80(\mathrm{~m}, 2 \mathrm{H}), 1.70-1.56(\mathrm{~m}, 3 \mathrm{H}), 1.38-1.13(\mathrm{~m}, 3 \mathrm{H})$.

${ }^{13} \mathrm{C}$ NMR $\left(126 \mathrm{MHz}, \mathrm{CDCl}_{3}, 25{ }^{\circ} \mathrm{C}, \delta\right): 137.9,135.3,120.9,116.4,55.2,45.7,36.4,29.1,25.0,24.6$.

HRMS-ESI(m/z) calc'd for $\mathrm{C}_{12} \mathrm{H}_{22} \mathrm{~N}[\mathrm{M}+1]^{+}, 180.1746$; found, 180.1747; deviation: 0.35 ppm.

N-(2-(Bicyclo[2.2.1]heptan-2-ylidene)ethyl)cyclohexanamine 18

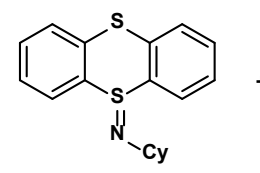

1

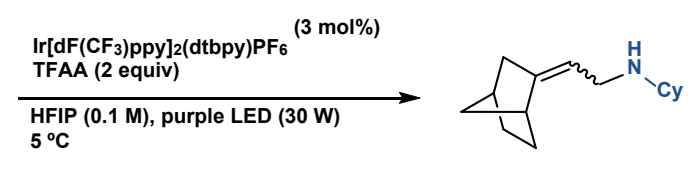

18

Under nitrogen atmosphere, to a 4-mL borosilicate vial equipped with a magnetic stir bar were added thianthrenylidene cyclohexylamine 1 (31.3 mg, $0.100 \mathrm{mmol}, 1.00$ equiv.), 2-vinylnorbornane (18.3 $\mathrm{mg}, 0.150$ mmol, 1.50 equiv.), Ir[dF(CF $)$ ppy $]_{2}(\mathrm{dtbpy}) \mathrm{PF}_{6}(3.3 \mathrm{mg}, 3.0 \mu \mathrm{mol}, 3.0 \mathrm{~mol} \%)$, HFIP $(1 \mathrm{~mL}, \mathrm{c}=0.1 \mathrm{M})$, and trifluoroacetic anhydride ( $28 \mu \mathrm{L}, 42 \mathrm{mg}, 0.20 \mathrm{mmol}, 2.0$ equiv.). The vial was sealed with a septum-cap and irradiated for $3 \mathrm{~h}$ at $5{ }^{\circ} \mathrm{C}$ using a purple LED $(30 \mathrm{~W})$. Then, the reaction mixture was concentrated. The residue was dissolved in DCM $(5 \mathrm{~mL})$ and washed with saturated sodium carbonate aqueous solution $(5 \mathrm{~mL})$. The aqueous phase was extracted with DCM $(2 \times 5 \mathrm{~mL})$. The organic phase was dried over $\mathrm{Na}_{2} \mathrm{SO}_{4}$ and the solvent was removed under reduced pressure. The residue was purified by chromatography on silica gel eluting with $\mathrm{CHCl}_{3} / \mathrm{MeOH}(50 / 1-30 / 1(\mathrm{v} / \mathrm{v}))$ to afford $14.0 \mathrm{mg}$ of 18 as a colorless oil (64\% yield, $\left.\mathrm{E}: Z=1: 1\right)$.

$\mathbf{R}_{\boldsymbol{f}}=0.25\left(\mathrm{CHCl}_{3} / \mathrm{MeOH}=15 / 1(\mathrm{v} / \mathrm{v})\right)$.

\section{NMR Spectroscopy:}

${ }^{1} \mathrm{H}$ NMR $\left(500 \mathrm{MHz}, \mathrm{CDCl}_{3}, 25^{\circ} \mathrm{C}, \delta\right): 5.46(\mathrm{t}, J=7.3 \mathrm{~Hz}, 1 \mathrm{H}), 5.25(\mathrm{t}, J=8.1 \mathrm{~Hz}, 1 \mathrm{H}), 3.57(\mathrm{t}, J=7.0 \mathrm{~Hz}$, 2H), 3.42 (dd, J = 11.4, 7.3 Hz, 2H), $2.95-2.82(\mathrm{~m}, 4 \mathrm{H}), 2.42(\mathrm{~s}, 1 \mathrm{H}), 2.35(\mathrm{~s}, 1 \mathrm{H}), 2.23-2.04(\mathrm{~m}, 7 \mathrm{H})$, 
$1.95-1.76(\mathrm{~m}, 7 \mathrm{H}), 1.74-1.55(\mathrm{~m}, 10 \mathrm{H}), 1.34-1.14(\mathrm{~m}, 12 \mathrm{H})$.

${ }^{13} \mathrm{C}$ NMR $\left(126 \mathrm{MHz}, \mathrm{CDCl}_{3}, 25^{\circ} \mathrm{C}, \delta\right): 156.0,155.3,107.6,107.1,55.6,54.9,45.7,42.9,42.6,40.8$,

39.3, 39.1, 39.0, 36.4, 36.1, 36.1, 29.4, 29.4, 29.4, 29.3, 28.9, 28.4, 25.0, 24.7, 24.7, 24.7.

HRMS-ESI(m/z) calc'd for $\mathrm{C}_{15} \mathrm{H}_{26} \mathrm{~N}$ [M+1] $]^{+}, 220.2061$; found, 220.2060; deviation: $-0.66 \mathrm{ppm}$.

N-(2-Cyclohexylideneethyl)cyclohexanamine 19

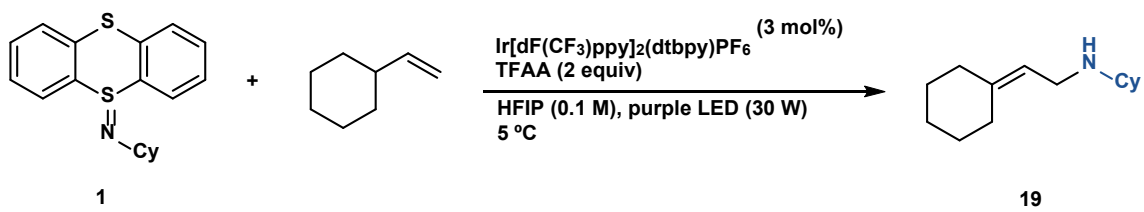

Under nitrogen atmosphere, to a 4-mL borosilicate vial equipped with a magnetic stir bar were added thianthrenylidene cyclohexylamine 1 (31.3 mg, $0.100 \mathrm{mmol}, 1.00$ equiv.), vinylcyclohexane (21.0 $\mathrm{hL}, 16.5 \mathrm{mg}$, $0.150 \mathrm{mmol}, 1.50$ equiv.), $\operatorname{Ir}\left[\mathrm{dF}\left(\mathrm{CF}_{3}\right) \mathrm{ppy}_{2}(\mathrm{dtbpy}) \mathrm{PF}_{6}(3.3 \mathrm{mg}, 3.0 \mu \mathrm{mol}, 3.0 \mathrm{~mol} \%), \mathrm{HFIP}(1 \mathrm{~mL}, \mathrm{c}=0.1 \mathrm{M})\right.$, and trifluoroacetic anhydride ( $28 \mu \mathrm{L}, 42 \mathrm{mg}, 0.20 \mathrm{mmol}, 2.0$ equiv.). The vial was sealed with a septum-cap and irradiated for $3 \mathrm{~h}$ at $5{ }^{\circ} \mathrm{C}$ using a purple LED $(30 \mathrm{~W})$. Then, the reaction mixture was concentrated. The residue was dissolved in DCM $(5 \mathrm{~mL})$ and washed with saturated sodium carbonate aqueous solution $(5 \mathrm{~mL})$. The aqueous phase was extracted with DCM $(2 \times 5 \mathrm{~mL})$. The organic phase was dried over $\mathrm{Na}_{2} \mathrm{SO}_{4}$ and the solvent was removed under reduced pressure. The residue was purified by chromatography on silica gel eluting with $\mathrm{CHCl}_{3} / \mathrm{MeOH}(50 / 1-30 / 1(\mathrm{v} / \mathrm{v}))$ to afford $10.6 \mathrm{mg}$ of 19 as a colorless oil (51\% yield).

$\mathbf{R}_{\boldsymbol{f}}=0.25\left(\mathrm{CHCl}_{3} / \mathrm{MeOH}=15 / 1(\mathrm{v} / \mathrm{v})\right)$.

\section{NMR Spectroscopy:}

${ }^{1} \mathrm{H}$ NMR (500 MHz, $\left.\mathrm{CDCl}_{3}, 25^{\circ} \mathrm{C}, \delta\right): 5.41(\mathrm{t}, J=7.5 \mathrm{~Hz}, 1 \mathrm{H}), 3.61(\mathrm{~d}, J=7.5 \mathrm{~Hz}, 2 \mathrm{H}), 3.03-2.84(\mathrm{~m}$, $1 \mathrm{H}), 2.30-2.05(\mathrm{~m}, 5 \mathrm{H}), 1.90-1.80(\mathrm{~m}, 2 \mathrm{H}), 1.69-1.52(\mathrm{~m}, 9 \mathrm{H}), 1.31-1.16(\mathrm{~m}, 4 \mathrm{H})$.

${ }^{13} \mathrm{C}$ NMR $\left(126 \mathrm{MHz}, \mathrm{CDCl}_{3}, 25{ }^{\circ} \mathrm{C}, \delta\right): 149.3,111.2,55.1,40.8,37.2,29.4,29.3,28.4,27.8,26.6,25.0$, 24.7.

HRMS-ESI(m/z) calc'd for $\mathrm{C}_{14} \mathrm{H}_{26} \mathrm{~N}$ [M+1] ${ }^{+}, 208.2059$; found, 208.2060; deviation: $0.16 \mathrm{ppm}$.

(Z)-N-(3-Phenylallyl)cyclohexanamine 20

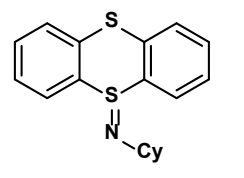

1

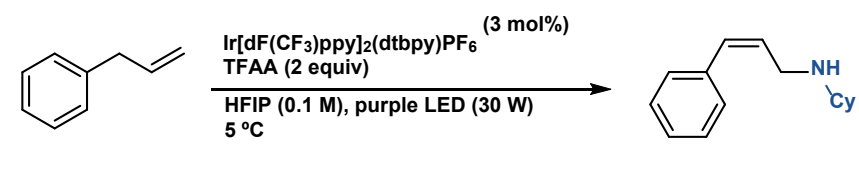

20

Under nitrogen atmosphere, to a 4-mL borosilicate vial equipped with a magnetic stir bar were added thianthrenylidene cyclohexylamine 1 (31.3 mg, $0.100 \mathrm{mmol}, 1.00$ equiv.), allylbenzene (20.0 $\mathrm{LL}, 17.7 \mathrm{mg}$, 0.150 mmol, 1.50 equiv.), $\operatorname{Ir}\left[\mathrm{dF}\left(\mathrm{CF}_{3}\right) \text { ppy }\right]_{2}(\mathrm{dtbpy}) \mathrm{PF}_{6}(3.3 \mathrm{mg}, 3.0 \mu \mathrm{mol}, 3.0 \mathrm{~mol} \%$ ), HFIP (1 mL, c = $0.1 \mathrm{M}$ ), and trifluoroacetic anhydride ( $28 \mu \mathrm{L}, 42 \mathrm{mg}, 0.20 \mathrm{mmol}, 2.0$ equiv.). The vial was sealed with a septum-cap 
and irradiated for $3 \mathrm{~h}$ at $5{ }^{\circ} \mathrm{C}$ using a purple LED $(30 \mathrm{~W})$. Then, the reaction mixture was concentrated. The residue was dissolved in DCM $(5 \mathrm{~mL})$ and washed with saturated sodium carbonate aqueous solution $(5 \mathrm{~mL})$. The aqueous phase was extracted with DCM $(2 \times 5 \mathrm{~mL})$. The organic phase was dried over $\mathrm{Na}_{2} \mathrm{SO}_{4}$ and the solvent was removed under reduced pressure. The residue was purified by chromatography on silica gel eluting with $\mathrm{CHCl}_{3} / \mathrm{MeOH}(50 / 1-30 / 1(\mathrm{v} / \mathrm{v}))$ to afford $17.2 \mathrm{mg}$ of 20 as a colorless oil ( $80 \%$ yield, $\left.\mathrm{E}: Z=1: 5\right)$.

$\mathbf{R}_{\boldsymbol{f}}=0.25\left(\mathrm{CHCl}_{3} / \mathrm{MeOH}=15 / 1(\mathrm{v} / \mathrm{v})\right)$.

\section{NMR Spectroscopy:}

${ }^{1} \mathrm{H}$ NMR $\left(500 \mathrm{MHz}, \mathrm{CDCl}_{3}, 25{ }^{\circ} \mathrm{C}, \delta\right): 7.36-7.30(\mathrm{~m}, 2 \mathrm{H}), 7.26-7.18(\mathrm{~m}, 3 \mathrm{H}), 6.51(\mathrm{~d}, J=11.7 \mathrm{~Hz}, 1 \mathrm{H})$, $5.77(\mathrm{dt}, J=11.7,6.5 \mathrm{~Hz}, 1 \mathrm{H}), 3.56(\mathrm{dd}, J=6.5,1.9 \mathrm{~Hz}, 2 \mathrm{H}), 2.46(\mathrm{tt}, J=10.5,3.7 \mathrm{~Hz}, 1 \mathrm{H}), 1.92-1.80$ $(\mathrm{m}, 2 \mathrm{H}), 1.77-1.67(\mathrm{~m}, 2 \mathrm{H}), 1.65-1.56(\mathrm{~m}, 2 \mathrm{H}), 1.29-1.19(\mathrm{~m}, 2 \mathrm{H}), 1.13-1.03(\mathrm{~m}, 2 \mathrm{H})$.

${ }^{13} \mathrm{C}$ NMR $\left(126 \mathrm{MHz}, \mathrm{CDCl}_{3}, 25{ }^{\circ} \mathrm{C}, \delta\right): 137.3,130.3,128.9,128.3,127.0,126.4,56.7,44.9,33.7,26.3$, 25.2 .

HRMS-ESI(m/z) calc'd for $\mathrm{C}_{15} \mathrm{H}_{22} \mathrm{~N}[\mathrm{M}+1]^{+}, 216.1747$; found, 216.1747; deviation: $-0.12 \mathrm{ppm}$.

(E)-N-(5-Octen-4-yl)cyclohexanamine 21

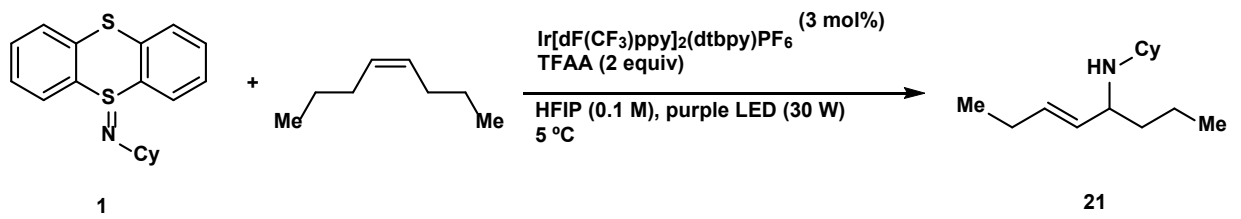

Under nitrogen atmosphere, to a 4-mL borosilicate vial equipped with a magnetic stir bar were added thianthrenylidene cyclohexylamine 1 (31.3 mg, $0.100 \mathrm{mmol}, 1.00$ equiv.), (Z)-4-octene (23.0 $\mu \mathrm{L}, 16.8 \mathrm{mg}$, $0.150 \mathrm{mmol}, 1.50$ equiv.), $\operatorname{Ir}\left[\mathrm{dF}\left(\mathrm{CF}_{3}\right) \mathrm{ppy}_{2}(\mathrm{dtbpy}) \mathrm{PF}_{6}(3.3 \mathrm{mg}, 3.0 \mu \mathrm{mol}, 3.0 \mathrm{~mol} \%), \mathrm{HFIP}(1 \mathrm{~mL}, \mathrm{c}=0.1 \mathrm{M})\right.$, and trifluoroacetic anhydride ( $28 \mu \mathrm{L}, 42 \mathrm{mg}, 0.20 \mathrm{mmol}, 2.0$ equiv.). The vial was sealed with a septum-cap and irradiated for $3 \mathrm{~h}$ at $5{ }^{\circ} \mathrm{C}$ using a purple LED $(30 \mathrm{~W})$. Then, the reaction mixture was concentrated. The residue was dissolved in DCM $(5 \mathrm{~mL})$ and washed with saturated sodium carbonate aqueous solution $(5 \mathrm{~mL})$. The aqueous phase was extracted with DCM $(2 \times 5 \mathrm{~mL})$. The organic phase was dried over $\mathrm{Na}_{2} \mathrm{SO}_{4}$ and the solvent was removed under reduced pressure. The residue was purified by chromatography on silica gel eluting with $\mathrm{CHCl}_{3} / \mathrm{MeOH}(50 / 1-30 / 1(\mathrm{v} / \mathrm{v}))$ to afford $12.7 \mathrm{mg}$ of 21 as a colorless oil (61\% yield, $\left.\mathrm{E}: \mathrm{Z}=15: 1\right)$.

Under nitrogen atmosphere, to a 4-mL borosilicate vial equipped with a magnetic stir bar were added thianthrenylidene cyclohexylamine 1 (31.3 mg, $0.100 \mathrm{mmol}, 1.00$ equiv.), (Z)-4-octene (15.0 $\mu \mathrm{L}, 11.2 \mathrm{mg}$, $0.100 \mathrm{mmol}, 1.00$ equiv.), $\operatorname{Ir}\left[\mathrm{dF}\left(\mathrm{CF}_{3}\right) \mathrm{ppy}_{2}(\mathrm{dtbpy}) \mathrm{PF}_{6}(3.3 \mathrm{mg}, 3.0 \mu \mathrm{mol}, 3.0 \mathrm{~mol} \%\right.$ ), HFIP (1 mL, c = $0.1 \mathrm{M})$, and trifluoroacetic anhydride ( $28 \mu \mathrm{L}, 42 \mathrm{mg}, 0.20 \mathrm{mmol}, 2.0$ equiv.). The vial was sealed with a septum-cap and irradiated for $3 \mathrm{~h}$ at $5{ }^{\circ} \mathrm{C}$ using a purple LED $(30 \mathrm{~W})$. Then, the reaction mixture was concentrated. The residue was dissolved in DCM $(5 \mathrm{~mL})$ and washed with saturated sodium carbonate aqueous solution $(5 \mathrm{~mL})$. The aqueous phase was extracted with DCM $(2 \times 5 \mathrm{~mL})$. The organic phase was dried over $\mathrm{Na}_{2} \mathrm{SO}_{4}$ and the solvent was removed under reduced pressure. The residue was purified by chromatography on silica gel 
eluting with $\mathrm{CHCl}_{3} / \mathrm{MeOH}(50 / 1-30 / 1(\mathrm{v} / \mathrm{v}))$ to afford $7.3 \mathrm{mg}$ of 21 as a colorless oil (35\% yield, $\left.\mathrm{E}: \mathrm{Z}=15: 1\right)$.

$\mathbf{R} f=0.25\left(\mathrm{CHCl}_{3} / \mathrm{MeOH}=15 / 1(\mathrm{v} / \mathrm{v})\right)$.

\section{NMR Spectroscopy:}

${ }^{1} \mathrm{H}$ NMR (500 MHz, $\left.\mathrm{CDCl}_{3}, 25{ }^{\circ} \mathrm{C}, \delta\right): 5.76(\mathrm{dt}, J=15.3,6.5 \mathrm{~Hz}, 1 \mathrm{H}), 5.48(\mathrm{dd}, J=15.4,9.7 \mathrm{~Hz}, 1 \mathrm{H}), 3.65$ $-3.44(\mathrm{~m}, 1 \mathrm{H}), 3.10-2.86(\mathrm{~m}, 1 \mathrm{H}), 2.34(\mathrm{~d}, J=10.9 \mathrm{~Hz}, 1 \mathrm{H}), 2.19-2.08(\mathrm{~m}, 2 \mathrm{H}), 2.02(\mathrm{~d}, J=12.2 \mathrm{~Hz}$, $1 \mathrm{H}), 1.93(\mathrm{ddd}, J=15.7,11.7,4.6 \mathrm{~Hz}, 1 \mathrm{H}), 1.88-1.74(\mathrm{~m}, 3 \mathrm{H}), 1.68-1.55(\mathrm{~m}, 3 \mathrm{H}), 1.34-1.17(\mathrm{~m}$, $5 \mathrm{H}), 1.01(\mathrm{t}, J=7.4 \mathrm{~Hz}, 3 \mathrm{H}), 0.89(\mathrm{t}, J=7.3 \mathrm{~Hz}, 3 \mathrm{H})$.

${ }^{13} \mathrm{C}$ NMR $\left(126 \mathrm{MHz}, \mathrm{CDCl}_{3}, 25{ }^{\circ} \mathrm{C}, \delta\right): 141.0,123.8,58.9,54.8,34.4,30.6,27.8,25.5,25.0,24.9,24.8$, $19.5,13.7,13.6$.

HRMS-ESI(m/z) calc'd for $\mathrm{C}_{14} \mathrm{H}_{28} \mathrm{~N}[\mathrm{M}+1]^{+}, 210.2216$; found, 210.2216; deviation: -0.03 ppm.

(E)-N-(5-Octen-4-yl)cyclohexanamine 21

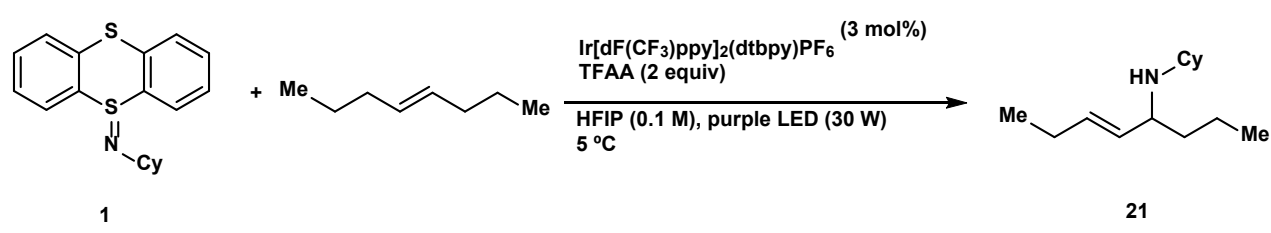

Under nitrogen atmosphere, to a 4-mL borosilicate vial equipped with a magnetic stir bar were added thianthrenylidene cyclohexylamine 1 (31.3 mg, $0.100 \mathrm{mmol}, 1.00$ equiv.), (E)-4-octene (23.0 $\mu \mathrm{L}, 16.8 \mathrm{mg}$, $0.150 \mathrm{mmol}, 1.50$ equiv.), $\operatorname{Ir}\left[\mathrm{dF}\left(\mathrm{CF}_{3}\right) \mathrm{ppy}_{2}(\mathrm{dtbpy}) \mathrm{PF}_{6}(3.3 \mathrm{mg}, 3.0 \mu \mathrm{mol}, 3.0 \mathrm{~mol} \%), \mathrm{HFIP}(1 \mathrm{~mL}, \mathrm{c}=0.1 \mathrm{M})\right.$, and trifluoroacetic anhydride ( $28 \mu \mathrm{L}, 42 \mathrm{mg}, 0.20 \mathrm{mmol}, 2.0$ equiv.). The vial was sealed with a septum-cap and irradiated for $3 \mathrm{~h}$ at $5{ }^{\circ} \mathrm{C}$ using a purple LED $(30 \mathrm{~W})$. Then, the reaction mixture was concentrated. The residue was dissolved in DCM $(5 \mathrm{~mL})$ and washed with saturated sodium carbonate aqueous solution $(5 \mathrm{~mL})$. The aqueous phase was extracted with DCM $(2 \times 5 \mathrm{~mL})$. The organic phase was dried over $\mathrm{Na}_{2} \mathrm{SO}_{4}$ and the solvent was removed under reduced pressure. The residue was purified by chromatography on silica gel eluting with $\mathrm{CHCl}_{3} / \mathrm{MeOH}(50 / 1-30 / 1(\mathrm{v} / \mathrm{v}))$ to afford $11.7 \mathrm{mg}$ of 21 as a colorless oil (56\% yield, $\left.\mathrm{E}: \mathrm{Z}=15: 1\right)$.

$\mathbf{R}_{\boldsymbol{f}}=0.25\left(\mathrm{CHCl}_{3} / \mathrm{MeOH}=15 / 1(\mathrm{v} / \mathrm{v})\right)$.

\section{NMR Spectroscopy:}

${ }^{1} \mathrm{H}$ NMR $\left(500 \mathrm{MHz}, \mathrm{CDCl}_{3}, 25^{\circ} \mathrm{C}, \delta\right): 5.76(\mathrm{dt}, J=15.3,6.5 \mathrm{~Hz}, 1 \mathrm{H}), 5.48(\mathrm{dd}, J=15.4,9.7 \mathrm{~Hz}, 1 \mathrm{H}), 3.65$ $-3.44(\mathrm{~m}, 1 \mathrm{H}), 3.10-2.86(\mathrm{~m}, 1 \mathrm{H}), 2.34(\mathrm{~d}, J=10.9 \mathrm{~Hz}, 1 \mathrm{H}), 2.19-2.08(\mathrm{~m}, 2 \mathrm{H}), 2.02(\mathrm{~d}, J=12.2 \mathrm{~Hz}$, $1 \mathrm{H}), 1.93(\mathrm{ddd}, J=15.7,11.7,4.6 \mathrm{~Hz}, 1 \mathrm{H}), 1.88-1.74(\mathrm{~m}, 3 \mathrm{H}), 1.68-1.55(\mathrm{~m}, 3 \mathrm{H}), 1.34-1.17(\mathrm{~m}$, $5 \mathrm{H}), 1.01(\mathrm{t}, J=7.4 \mathrm{~Hz}, 3 \mathrm{H}), 0.89(\mathrm{t}, J=7.3 \mathrm{~Hz}, 3 \mathrm{H})$.

${ }^{13} \mathrm{C}$ NMR $\left(126 \mathrm{MHz}, \mathrm{CDCl}_{3}, 25^{\circ} \mathrm{C}, \delta\right): 141.0,123.8,58.9,54.8,34.4,30.6,27.8,25.5,25.0,24.9,24.8$, 19.5, 13.7, 13.6.

HRMS-ESI(m/z) calc'd for $\mathrm{C}_{14} \mathrm{H}_{28} \mathrm{~N}[\mathrm{M}+1]^{+}, 210.2216$; found, 210.2216; deviation: -0.03 ppm. 

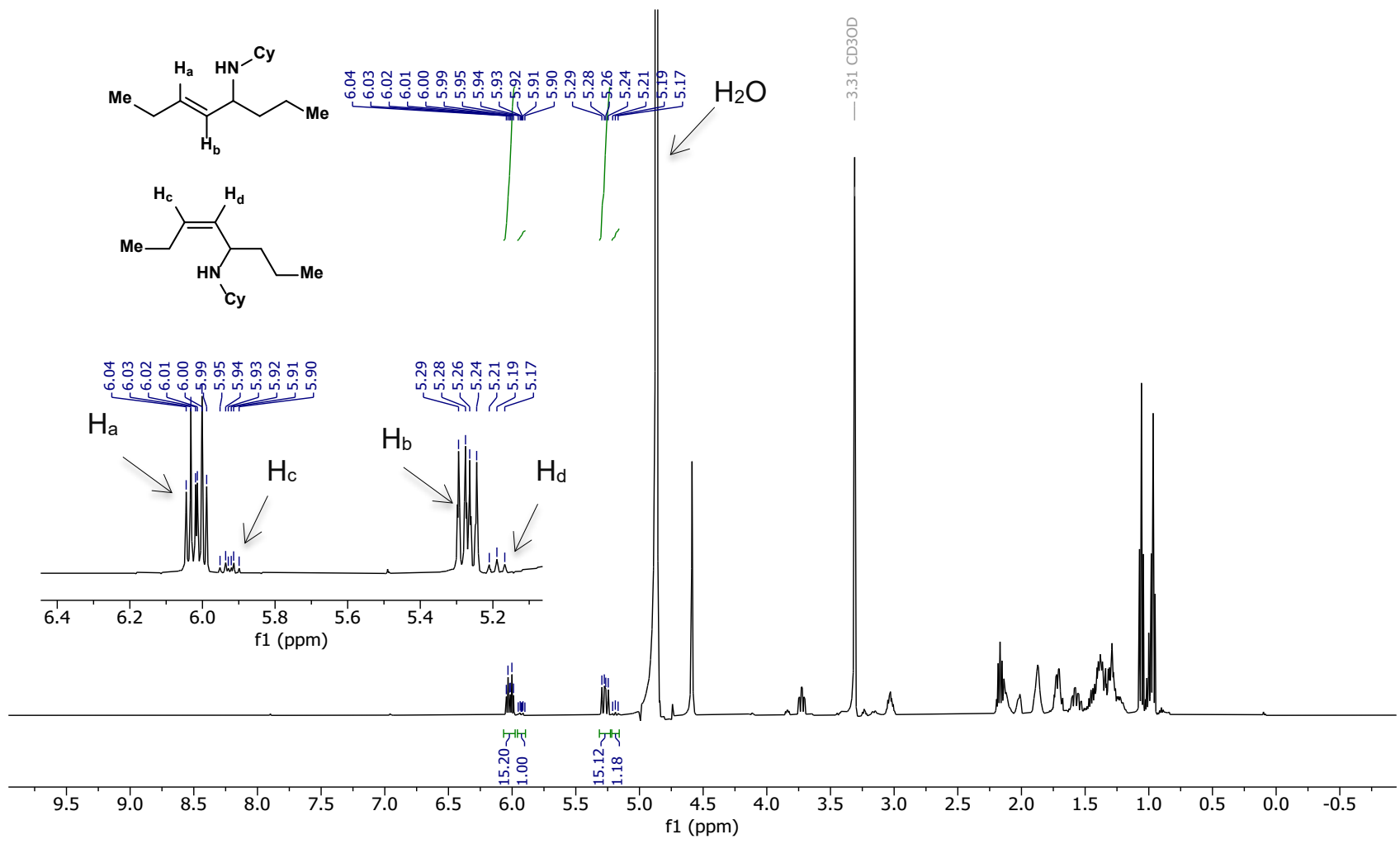

Figure S3. ${ }^{1} \mathrm{H}$ NMR of 21 in $\mathrm{MeOH}-d_{4}$. The ${ }^{1} \mathrm{H}$ NMR of 21 in $\mathrm{MeOH}-d_{4}$ clearly shows a cis-isomer: ${ }^{1} \mathrm{H}$ NMR $\left(500 \mathrm{MHz}, \mathrm{MeOH}-d_{4}, 25^{\circ} \mathrm{C}, \delta\right): 5.93(\mathrm{dt}, J=10.8,7.6 \mathrm{~Hz}, 1 \mathrm{H}), 5.19(\mathrm{dd}, J=11.0,10.5 \mathrm{~Hz}, 1 \mathrm{H})$. The E:Z ratio is determined according to the integration ratio for $H_{a}$ and $H_{c}$ as 15:1.

\section{6-(Cyclohexylamino)-3,7-dimethyloct-7-en-1-ol 22}

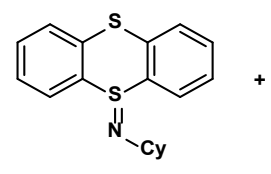

1

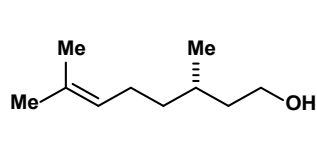

23, (S)-(-)- $\beta-$ Citronellol

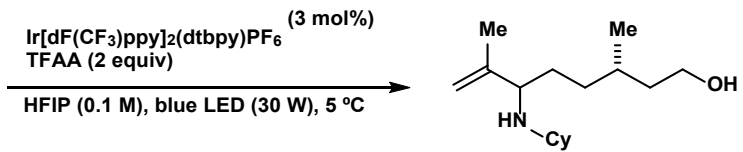

22

Under nitrogen atmosphere, to a 4-mL borosilicate vial equipped with a magnetic stir bar were added thianthrenylidene cyclohexylamine 1 (31.3 mg, 0.100 mmol, 1.00 equiv.), (S)-(-)- $\beta$-Citronellol (23.4 mg, 0.150 mmol, 1.50 equiv.), Ir[dF(CF $)$ ppy $]_{2}\left(\mathrm{dtbpy} \mathrm{PF}_{6}(3.3 \mathrm{mg}, 3.0 \mu \mathrm{mol}, 3.0 \mathrm{~mol} \%)\right.$, HFIP $(1 \mathrm{~mL}, \mathrm{c}=0.1 \mathrm{M})$, and trifluoroacetic anhydride ( $28 \mu \mathrm{L}, 42 \mathrm{mg}, 0.20 \mathrm{mmol}, 2.0$ equiv.). The vial was sealed with a septum-cap and irradiated for $3 \mathrm{~h}$ at $8{ }^{\circ} \mathrm{C}$ using a blue LED $(30 \mathrm{~W})$. Then, the reaction mixture was concentrated. The residue was dissolved in DCM $(5 \mathrm{~mL})$ and washed with saturated sodium carbonate aqueous solution $(5 \mathrm{~mL})$. The aqueous phase was extracted with DCM $(2 \times 5 \mathrm{~mL})$. The organic phase was dried over $\mathrm{Na}_{2} \mathrm{SO}_{4}$ and the solvent was removed under reduced pressure. The residue was purified by chromatography on silica gel eluting with $\mathrm{CHCl}_{3} / \mathrm{MeOH}(30 / 1-10 / 1(\mathrm{v} / \mathrm{v}))$ to afford $16.9 \mathrm{mg}$ of 22 as a colorless oil (67\% yield, 1:1 dr). Under nitrogen atmosphere, to a 4-mL borosilicate vial equipped with a magnetic stir bar were added thianthrenylidene cyclohexylamine 1 (31.3 mg, 0.100 mmol, 1.00 equiv.), (S)-(-)- $\beta$-Citronellol (15.6 mg, 0.100 
mmol, 1.00 equiv.), Ir[dF(CF $)$ ppy $]_{2}(\mathrm{dtbpy}) \mathrm{PF}_{6}(3.3 \mathrm{mg}, 3.0 \mu \mathrm{mol}, 3.0 \mathrm{~mol} \%), \mathrm{HFIP}(1 \mathrm{~mL}, \mathrm{c}=0.1 \mathrm{M})$, and trifluoroacetic anhydride ( $28 \mu \mathrm{L}, 42 \mathrm{mg}, 0.20 \mathrm{mmol}, 2.0$ equiv.). The vial was sealed with a septum-cap and irradiated for $3 \mathrm{~h}$ at $8{ }^{\circ} \mathrm{C}$ using a blue LED $(30 \mathrm{~W})$. Then, the reaction mixture was concentrated. The residue was dissolved in DCM $(5 \mathrm{~mL})$ and washed with saturated sodium carbonate aqueous solution $(5 \mathrm{~mL})$. The aqueous phase was extracted with DCM $(2 \times 5 \mathrm{~mL})$. The organic phase was dried over $\mathrm{Na}_{2} \mathrm{SO}_{4}$ and the solvent was removed under reduced pressure. The residue was purified by chromatography on silica gel eluting with $\mathrm{CHCl}_{3} / \mathrm{MeOH}(30 / 1-10 / 1(\mathrm{v} / \mathrm{v}))$ to afford $12.4 \mathrm{mg}$ of 22 as a colorless oil (49\% yield, 1:1 dr).

$\boldsymbol{R}_{\boldsymbol{f}}=0.15\left(\mathrm{CHCl}_{3} / \mathrm{MeOH}=10 / 1(\mathrm{v} / \mathrm{v})\right)$.

\section{NMR Spectroscopy:}

${ }^{1} \mathrm{H}$ NMR $\left(500 \mathrm{MHz}, \mathrm{CDCl}_{3}, 25^{\circ} \mathrm{C}, \delta\right): 4.85(\mathrm{~s}, 1 \mathrm{H}), 4.80(\mathrm{~s}, 1 \mathrm{H}), 3.73-3.58(\mathrm{~m}, 2 \mathrm{H}), 3.26-3.14(\mathrm{~m}, 1 \mathrm{H})$, $2.41-2.28(\mathrm{~m}, 1 \mathrm{H}), 1.93(\mathrm{~d}, J=12.2 \mathrm{~Hz}, 1 \mathrm{H}), 1.76-1.66(\mathrm{~m}, 3 \mathrm{H}), 1.61(\mathrm{~s}, 3 \mathrm{H}), 1.61-1.52(\mathrm{~m}, 3 \mathrm{H})$, $1.47-1.33(\mathrm{~m}, 3 \mathrm{H}), 1.31-1.09(\mathrm{~m}, 7 \mathrm{H}), 0.88(\mathrm{dd}, J=6.4,2.3 \mathrm{~Hz}, 3 \mathrm{H})$.

${ }^{13} \mathrm{C}$ NMR $\left(126 \mathrm{MHz}, \mathrm{CDCl}_{3}, 2{ }^{\circ} \mathrm{C}, \delta\right): 138.3,138.2,120.0,62.1,61.6,60.8,60.6,55.2,39.4,38.6,32.8$, 32.5, 30.5, 30.4, 29.3, 28.6, 28.0, 28.0, 27.7, 26.9, 25.0, 24.9, 24.6, 20.1, 19.7, 16.84, 16.76.

HRMS-ESI(m/z) calc'd for $\mathrm{C}_{13} \mathrm{H}_{24} \mathrm{NO}_{2}[\mathrm{M}+1]^{+}, 254.2478$; found, 254.2478; deviation: 0.07 ppm.

(Z)-N-(4-phenylbut-3-en-2-yl)cyclohexanamine 24

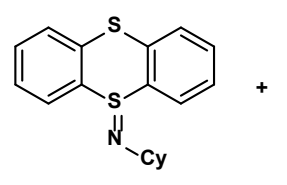

1

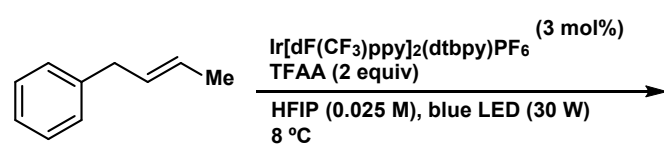
$8{ }^{\circ} \mathrm{C}$

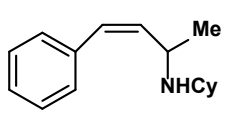

24

Under nitrogen atmosphere, to a 4-mL borosilicate vial equipped with a magnetic stir bar were added thianthrenylidene cyclohexylamine 1 (31.3 mg, $0.100 \mathrm{mmol}, 1.00$ equiv.), (E)-1-phenyl-2-butene (19.8 mg, $0.150 \mathrm{mmol}, 1.50$ equiv.), $\operatorname{Ir}\left[\mathrm{dF}\left(\mathrm{CF}_{3}\right) \mathrm{ppy}_{2}(\mathrm{dtbpy}) \mathrm{PF}_{6}(3.3 \mathrm{mg}, 3.0 \mu \mathrm{mol}, 3.0 \mathrm{~mol} \%), \mathrm{HFIP}(1 \mathrm{~mL}, \mathrm{c}=0.1 \mathrm{M})\right.$, and trifluoroacetic anhydride ( $28 \mu \mathrm{L}, 42 \mathrm{mg}, 0.20 \mathrm{mmol}, 2.0$ equiv.). The vial was sealed with a septum-cap and irradiated for $3 \mathrm{~h}$ at $8{ }^{\circ} \mathrm{C}$ using a blue LED $(30 \mathrm{~W})$. Then, the reaction mixture was concentrated. The residue was dissolved in DCM $(5 \mathrm{~mL})$ and washed with saturated sodium carbonate aqueous solution $(5 \mathrm{~mL})$. The aqueous phase was extracted with DCM $(2 \times 5 \mathrm{~mL})$. The organic phase was dried over $\mathrm{Na}_{2} \mathrm{SO}_{4}$ and the solvent was removed under reduced pressure. The residue was purified by chromatography on silica gel eluting with $\mathrm{CHCl}_{3} / \mathrm{MeOH}(30 / 1-10 / 1(\mathrm{v} / \mathrm{v}))$ to afford $13.2 \mathrm{mg}$ of 24 as a colorless oil (58\% yield, $\left.\mathrm{E}: \mathrm{Z}=6: 1\right)$.

$\mathbf{R}_{\boldsymbol{f}}=0.30\left(\mathrm{CHCl}_{3} / \mathrm{MeOH}=20 / 1(\mathrm{v} / \mathrm{v})\right)$.

\section{NMR Spectroscopy:}

${ }^{1} \mathrm{H}$ NMR $\left(500 \mathrm{MHz}, \mathrm{CDCl}_{3}, 25^{\circ} \mathrm{C}, \delta\right): 7.33(\mathrm{t}, J=7.6 \mathrm{~Hz}, 2 \mathrm{H}), 7.25-7.20(\mathrm{~m}, 3 \mathrm{H}), 6.51(\mathrm{~d}, J=11.6 \mathrm{~Hz}$, $1 \mathrm{H}), 5.49(\mathrm{dd}, J=11.6,9.6 \mathrm{~Hz}, 1 \mathrm{H}), 3.95(\mathrm{dd}, J=9.7,6.3 \mathrm{~Hz}, 1 \mathrm{H}), 2.43(\mathrm{t}, J=10.6 \mathrm{~Hz}, 1 \mathrm{H}), 1.82-1.76$ $(\mathrm{m}, 1 \mathrm{H}), 1.71-1.65(\mathrm{~m}, 1 \mathrm{H}), 1.58-1.51(\mathrm{~m}, 3 \mathrm{H}), 1.26(\mathrm{~d}, \mathrm{~J}=3.7 \mathrm{~Hz}, 3 \mathrm{H}), 1.24-1.17(\mathrm{~m}, 1 \mathrm{H}), 1.10-$ $1.04(\mathrm{~m}, 3 \mathrm{H}), 0.91-0.81(\mathrm{~m}, 1 \mathrm{H})$. 
${ }^{13} \mathrm{C}$ NMR $\left(126 \mathrm{MHz}, \mathrm{CDCl}_{3}, 25^{\circ} \mathrm{C}, \delta\right): 135.7,134.9,129.0,128.9,128.2,128.1,127.8,127.0,55.0,48.1$, $30.2,27.7,24.9,24.8,24.5,18.8$.

HRMS-ESI(m/z) calc'd for $\mathrm{C}_{18} \mathrm{H}_{32} \mathrm{NO}_{2}[\mathrm{M}+1]^{+}, 229.1825$; found, 229.1825; deviation: $-0.14 \mathrm{ppm}$.

(E)-N-(Oct-3-en-2-yl)cyclohexanamine 25

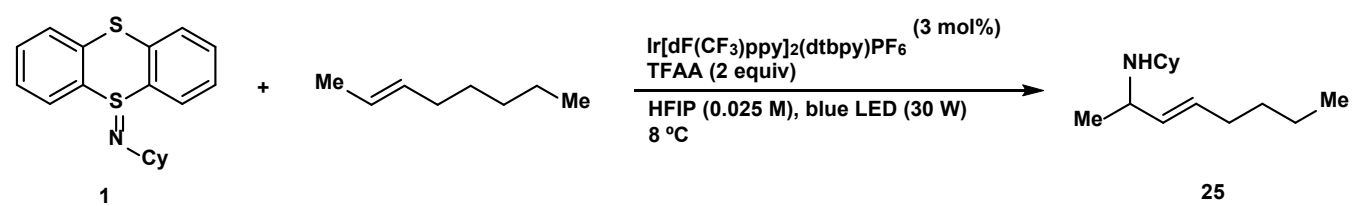

Under nitrogen atmosphere, to a 4-mL borosilicate vial equipped with a magnetic stir bar were added thianthrenylidene cyclohexylamine 1 (31.3 mg, 0.100 mmol, 1.00 equiv.), (E) -2-octene (23.4 $\mu \mathrm{L}, 16.8 \mathrm{mg}$, 0.150 mmol, 1.50 equiv.), Ir[dF(CF3)ppy]2(dtbpy)PF6 (3.3 mg, $3.0 \mu \mathrm{mol}, 3.0 \mathrm{~mol} \%)$, HFIP (1 mL, c = $0.1 \mathrm{M}$ ), and trifluoroacetic anhydride ( $28 \mu \mathrm{L}, 42 \mathrm{mg}, 0.20 \mathrm{mmol}, 2.0$ equiv.). The vial was sealed with a septum-cap and irradiated for $3 \mathrm{~h}$ at $8{ }^{\circ} \mathrm{C}$ using a blue LED $(30 \mathrm{~W})$. Then, the reaction mixture was concentrated. The residue was dissolved in DCM $(5 \mathrm{~mL})$ and washed with saturated sodium carbonate aqueous solution $(5 \mathrm{~mL})$. The aqueous phase was extracted with DCM $(2 \times 5 \mathrm{~mL})$. The organic phase was dried over $\mathrm{Na}_{2} \mathrm{SO}_{4}$ and the solvent was removed under reduced pressure. The residue was purified by chromatography on silica gel eluting with $\mathrm{CHCl}_{3} / \mathrm{MeOH}(30 / 1-10 / 1(\mathrm{v} / \mathrm{v}))$ to afford $10.4 \mathrm{mg}$ of 25 as a colorless oil $(50 \%$ yield, $\mathrm{E}: \mathrm{Z}=15: 1)$.

$\boldsymbol{R}_{\boldsymbol{f}}=0.20\left(\mathrm{CHCl}_{3} / \mathrm{MeOH}=20 / 1(\mathrm{v} / \mathrm{v})\right)$.

\section{NMR Spectroscopy:}

${ }^{1} \mathrm{H}$ NMR $\left(500 \mathrm{MHz}, \mathrm{CDCl}_{3}, 25^{\circ} \mathrm{C}, \delta\right): 5.73(\mathrm{dt}, J=14.3,6.8 \mathrm{~Hz}, 1 \mathrm{H}), 5.53(\mathrm{dd}, J=15.1,9.2 \mathrm{~Hz}, 1 \mathrm{H}), 3.77$ $-3.74(\mathrm{~m}, 1 \mathrm{H}), 2.98-2.88(\mathrm{~m}, 1 \mathrm{H}), 2.07(\mathrm{q}, J=7.1 \mathrm{~Hz}, 2 \mathrm{H}), 2.03-1.95(\mathrm{~m}, 1 \mathrm{H}), 1.91-1.76(\mathrm{~m}, 3 \mathrm{H})$, $1.61(\mathrm{~d}, J=6.3 \mathrm{~Hz}, 2 \mathrm{H}), 1.44(\mathrm{~d}, J=6.8 \mathrm{~Hz}, 3 \mathrm{H}), 1.37-1.14(\mathrm{~m}, 9 \mathrm{H}), 0.89(\mathrm{t}, J=7.1 \mathrm{~Hz}, 3 \mathrm{H})$.

${ }^{13} \mathrm{C}$ NMR $\left(126 \mathrm{MHz} \mathrm{CDCl}_{3}, 25{ }^{\circ} \mathrm{C}, \delta\right): 137.9,126.0,54.5,53.8,31.9,31.2,30.2,27.8,25.1,24.9,24.7$, $22.3,19.3,14.0$.

HRMS-ESI(m/z) calc'd for $\mathrm{C}_{18} \mathrm{H}_{32} \mathrm{NO}_{2}[\mathrm{M}+1]^{+}, 209.2138$; found, 209.2138; deviation: $-0.10 \mathrm{ppm}$.

N-(Oct-1-en-3-yl)cyclohexanamine 25,

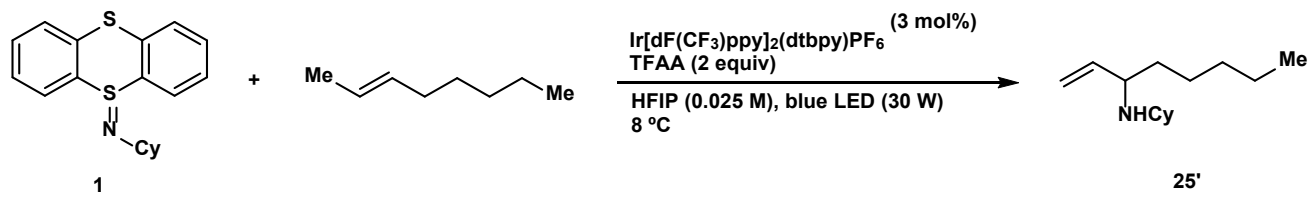

Under nitrogen atmosphere, to a 4-mL borosilicate vial equipped with a magnetic stir bar were added thianthrenylidene cyclohexylamine 1 (31.3 mg, $0.100 \mathrm{mmol}, 1.00$ equiv.), (E) -2-octene (23.4 $\mu \mathrm{L}, 16.8 \mathrm{mg}$, $0.150 \mathrm{mmol}, 1.50$ equiv.), $\operatorname{Ir}\left[\mathrm{dF}\left(\mathrm{CF}_{3}\right) \mathrm{ppy}_{2}(\mathrm{dtbpy}) \mathrm{PF}_{6}(3.3 \mathrm{mg}, 3.0 \mu \mathrm{mol}, 3.0 \mathrm{~mol} \%), \mathrm{HFIP}(1 \mathrm{~mL}, \mathrm{c}=0.1 \mathrm{M})\right.$, and trifluoroacetic anhydride ( $28 \mu \mathrm{L}, 42 \mathrm{mg}, 0.20 \mathrm{mmol}, 2.0$ equiv.). The vial was sealed with a septum-cap 
and irradiated for $3 \mathrm{~h}$ at $8{ }^{\circ} \mathrm{C}$ using a blue LED $(30 \mathrm{~W})$. Then, the reaction mixture was concentrated. The residue was dissolved in DCM $(5 \mathrm{~mL})$ and washed with saturated sodium carbonate aqueous solution $(5 \mathrm{~mL})$. The aqueous phase was extracted with DCM $(2 \times 5 \mathrm{~mL})$. The organic phase was dried over $\mathrm{Na}_{2} \mathrm{SO}_{4}$ and the solvent was removed under reduced pressure. The residue was purified by chromatography on silica gel eluting with $\mathrm{CHCl}_{3} / \mathrm{MeOH}(30 / 1-10 / 1(\mathrm{v} / \mathrm{v}))$ to afford $5.0 \mathrm{mg}$ of 25 ' as a colorless oil (24\% yield).

$\boldsymbol{R}_{\boldsymbol{f}}=0.25\left(\mathrm{CHCl}_{3} / \mathrm{MeOH}=20 / 1(\mathrm{v} / \mathrm{v})\right)$.

\section{NMR Spectroscopy:}

${ }^{1} \mathrm{H}$ NMR $\left(500 \mathrm{MHz}, \mathrm{CDCl}_{3}, 25{ }^{\circ} \mathrm{C}, \delta\right): 5.89(\mathrm{dt}, J=16.4,9.9 \mathrm{~Hz}, 1 \mathrm{H}), 5.44(\mathrm{~d}, J=10.3 \mathrm{~Hz}, 1 \mathrm{H}), 5.32(\mathrm{~d}, J$ $=17.1 \mathrm{~Hz}, 1 \mathrm{H}), 3.56(\mathrm{~d}, J=10.4 \mathrm{~Hz}, 1 \mathrm{H}), 2.99(\mathrm{~d}, J=10.9 \mathrm{~Hz}, 1 \mathrm{H}), 2.31(\mathrm{~d}, J=12.6 \mathrm{~Hz}, 1 \mathrm{H}), 2.17-$ $2.11(\mathrm{~m}, 1 \mathrm{H}), 2.11-2.01(\mathrm{~m}, 1 \mathrm{H}), 1.87-1.57(\mathrm{~m}, 10 \mathrm{H}), 1.34-1.17(\mathrm{~m}, 5 \mathrm{H}), 0.85(\mathrm{t}, J=6.5 \mathrm{~Hz}, 3 \mathrm{H})$.

HRMS-ESI(m/z) calc'd for $\mathrm{C}_{18} \mathrm{H}_{32} \mathrm{NO}_{2}$ [M+1] ${ }^{+}$, 209.2138; found, 209.2138; deviation: 0.09 ppm.

(E)-3,7-dimethylocta-2,6-dien-1-yl acetate 27

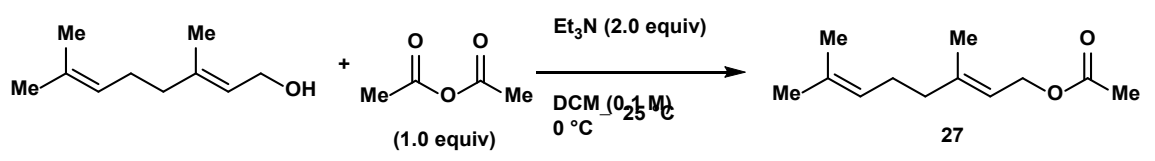

Under air, to a $50-\mathrm{mL}$ flask equipped with a magnetic stir bar were added geraniol $(568 \mu \mathrm{L}, 500 \mathrm{mg}, 3.24$ mmol, 1.00 equiv.) and DCM (32 mL, c = $0.10 \mathrm{M})$. The flask was sealed with a rubber septum equipped with an argon balloon, and the reaction mixture was chilled in an ice-water cooling bath. Triethylamine $(903 \mu \mathrm{L}$, $656 \mathrm{mg}, 6.48 \mathrm{mmol}, 2.00$ equiv.) was slowly added into the reaction mixture while cooling, and subsequently, acetic anhydride ( $306 \mu \mathrm{L}, 331 \mathrm{mg}, 3.24 \mathrm{mmol}, 1.00$ equiv.) was added dropwise within $2 \mathrm{~min}$. After addition, the reaction mixture was stirred at $0{ }^{\circ} \mathrm{C}$ for $30 \mathrm{~min}$, then at $25^{\circ} \mathrm{C}$ for $15 \mathrm{~h}$. Then, $20 \mathrm{~mL}$ saturated aqueous $\mathrm{NH}_{4} \mathrm{Cl}$ was added into the reaction mixture. The organic phase was separated, and the aqueous phase was extracted with DCM $(3 \times 15 \mathrm{~mL})$. The combined organic phase was dried over $\mathrm{MgSO}_{4}$ and the solvent was removed under reduced pressure. The residue was purified by chromatography on silica gel eluting with hexanes/EtOAc (50/1-30/1 (v/v)) to afford $595 \mathrm{mg}$ of the acylated product 27 as a colorless liquid (93\% yield).

$\mathbf{R}_{\boldsymbol{f}}=0.65($ hexanes $/$ EtOAc $=10 / 1(\mathrm{v} / \mathrm{v}))$.

\section{NMR Spectroscopy:}

${ }^{1} \mathrm{H}$ NMR $\left(500 \mathrm{MHz}, \mathrm{CDCl}_{3}, 25{ }^{\circ} \mathrm{C}, \delta\right): 5.34$ (ddt, $\left.J=7.2,5.8,1.3 \mathrm{~Hz}, 1 \mathrm{H}\right), 5.08$ (dddd, $J=7.0,5.5,2.9$, $1.5 \mathrm{~Hz}, 1 \mathrm{H}), 4.58(\mathrm{~d}, J=6.8 \mathrm{f}, 2 \mathrm{H}), 2.13-2.07(\mathrm{~m}, 2 \mathrm{H}), 2.05(\mathrm{~s}, 3 \mathrm{H}), 2.06-2.01(\mathrm{~m}, 2 \mathrm{fH}), 1.70(\mathrm{~s}, 3 \mathrm{H})$, $1.68(\mathrm{~d}, J=1.4 \mathrm{~Hz}, 3 \mathrm{H}), 1.60(\mathrm{~s}, 3 \mathrm{H})$.

${ }^{13} \mathrm{C}$ NMR $\left(126 \mathrm{MHz}, \mathrm{CDCl}_{3}, 25^{\circ} \mathrm{C}, \delta\right): 171.5,142.7,132.2,124.1,118.6,61.8,39.9,26.7,26.1,21.5$, 18.1, 16.8.

Both NMR data are consistent with the reported data. ${ }^{3}$ 
(E)-6-(Cyclohexylamino)-3,7-dimethylocta-2,7-dien-1-yl acetate 26

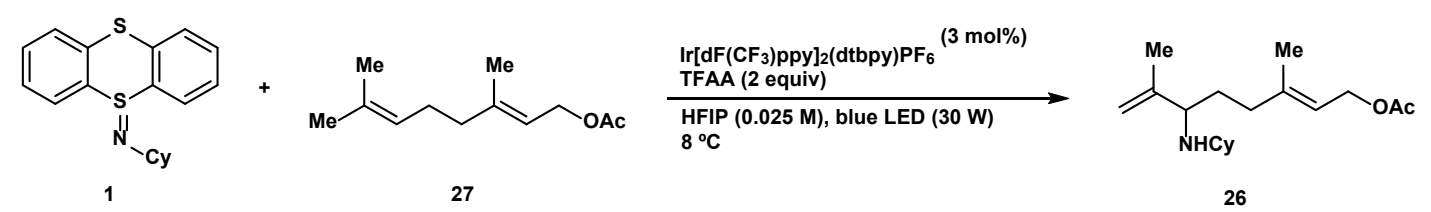

Under nitrogen atmosphere, to a 4-mL borosilicate vial equipped with a magnetic stir bar were added thianthrenylidene cyclohexylamine 1 (31.3 mg, 0.100 mmol, 1.00 equiv.), 27 (29.4 mg, 0.150 mmol, 1.50 equiv.), $\operatorname{Ir}\left[\mathrm{dF}\left(\mathrm{CF}_{3}\right) \mathrm{ppy}_{2}(\mathrm{dtbpy}) \mathrm{PF}_{6}(3.3 \mathrm{mg}, 3.0 \mu \mathrm{mol}, 3.0 \mathrm{~mol} \%\right.$ ), HFIP (1 mL, c = 0.1 M), and trifluoroacetic anhydride ( $28 \mu \mathrm{L}, 42 \mathrm{mg}, 0.20 \mathrm{mmol}, 2.0$ equiv.). The vial was sealed with a septum-cap and irradiated for $3 \mathrm{~h}$ at $8{ }^{\circ} \mathrm{C}$ using a blue LED $(30 \mathrm{~W})$. Then, the reaction mixture was concentrated. The residue was dissolved in DCM $(5 \mathrm{~mL})$ and washed with saturated sodium carbonate aqueous solution $(5 \mathrm{~mL})$. The aqueous phase was extracted with DCM $(2 \times 5 \mathrm{~mL})$. The organic phase was dried over $\mathrm{Na}_{2} \mathrm{SO}_{4}$ and the solvent was removed under reduced pressure. The residue was purified by chromatography on silica gel eluting with $\mathrm{CHCl}_{3} / \mathrm{MeOH}(30 / 1-10 / 1(\mathrm{v} / \mathrm{v}))$ to afford $13.3 \mathrm{mg}$ of 26 as a colorless oil (45\% yield).

$\mathbf{R}_{\boldsymbol{f}}=0.30\left(\mathrm{CHCl}_{3} / \mathrm{MeOH}=20 / 1(\mathrm{v} / \mathrm{v})\right)$.

\section{NMR Spectroscopy:}

${ }^{1} \mathrm{H}$ NMR $\left(500 \mathrm{MHz}, \mathrm{CDCl}_{3}, 25^{\circ} \mathrm{C}, \delta\right): 5.33(\mathrm{t}, J=7.2 \mathrm{~Hz}, 1 \mathrm{H}), 5.14(\mathrm{~s}, 1 \mathrm{H}), 5.05(\mathrm{~s}, 1 \mathrm{H}), 4.54$ (d, J = 7.0 $\mathrm{Hz}, 2 \mathrm{H}), 3.59-3.56(\mathrm{~m}, 1 \mathrm{H}), 2.73-2.69(\mathrm{~m}, 1 \mathrm{H}), 2.16(\mathrm{~d}, \mathrm{~J}=12.4 \mathrm{~Hz}, 1 \mathrm{H}), 2.03(\mathrm{~s}, 3 \mathrm{H}), 1.99-1.88(\mathrm{~m}$, $3 \mathrm{H}), 1.82(\mathrm{~s}, 3 \mathrm{H}), 1.76-1.69(\mathrm{~m}, 1 \mathrm{H}), 1.68(\mathrm{~s}, 3 \mathrm{H}), 1.64-1.59(\mathrm{~m}, 2 \mathrm{H}), 1.52-1.41(\mathrm{~m}, 3 \mathrm{H}), 1.24-1.11$ $(\mathrm{m}, 4 \mathrm{H})$.

${ }^{13} \mathrm{C}$ NMR $\left(126 \mathrm{MHz}, \mathrm{CDCl}_{3}, 2{ }^{\circ} \mathrm{C}, \delta\right): 171.2,140.2,137.9,120.6,120.0,61.2,61.1,55.3,35.7,30.5$, $27.8,27.5,25.0,25.0,24.6,21.1,17.1,16.3$.

HRMS-ESI(m/z) calc'd for $\mathrm{C}_{18} \mathrm{H}_{32} \mathrm{NO}_{2}[\mathrm{M}+1]^{+}, 294.2428$; found, 294.2428; deviation: -0.29 ppm.

\section{4-(Cyclohexylamino)allylestrenol 28}

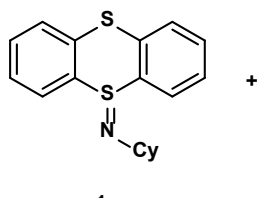

1

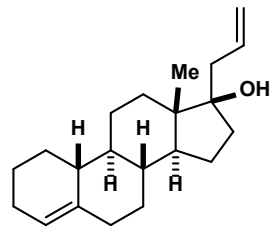

29

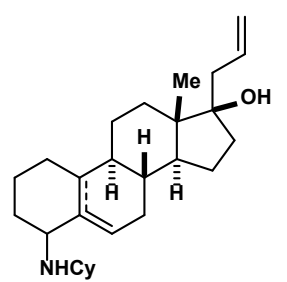

28

Under nitrogen atmosphere, to four 4-mL borosilicate vials equipped with magnetic stir bars were added in each vial thianthrenylidene cyclohexylamine 1 ( $7.8 \mathrm{mg}, 0.025 \mathrm{mmol}, 1.0$ equiv.), allylestrenol 29 (9.0 mg, $0.030 \mathrm{mmol}, 1.2$ equiv.), $\operatorname{Ir}\left[\mathrm{dF}\left(\mathrm{CF}_{3}\right) \mathrm{ppy}_{2}(\mathrm{dtbpy}) \mathrm{PF}_{6}(0.8 \mathrm{mg}, 0.8 \mu \mathrm{mol}, 3 \mathrm{~mol} \%), \mathrm{HFIP}(1 \mathrm{~mL}, \mathrm{c}=0.1 \mathrm{M})\right.$, and trifluoroacetic anhydride $(7.0 \mu \mathrm{L}, 11 \mathrm{mg}, 0.050 \mathrm{mmol}, 2.0$ equiv.). The vials were sealed with a septum-cap and irradiated for $3 \mathrm{~h}$ at $5{ }^{\circ} \mathrm{C}$ using a purple LED $(30 \mathrm{~W})$. Then, the reaction mixtures were combined and 
concentrated. The residue was dissolved in DCM $(5 \mathrm{~mL})$ and washed with saturated sodium carbonate aqueous solution $(5 \mathrm{~mL})$. The aqueous phase was extracted with DCM $(2 \times 5 \mathrm{~mL})$. The organic phase was dried over $\mathrm{Na}_{2} \mathrm{SO}_{4}$ and the solvent was removed under reduced pressure. The residue was purified by chromatography on silica gel eluting with $\mathrm{CHCl}_{3} / \mathrm{MeOH}(50 / 1-40 / 1(\mathrm{v} / \mathrm{v}))$ to afford $18.6 \mathrm{mg}$ of 28 as a colorless oil ( $47 \%$ yield, $1.2: 1)$.

$\boldsymbol{R}_{\boldsymbol{f}}=0.30\left(\mathrm{CHCl}_{3} / \mathrm{MeOH}=20 / 1(\mathrm{v} / \mathrm{v})\right)$.

\section{NMR Spectroscopy:}

${ }^{1} \mathrm{H}$ NMR (600 MHz, $\left.\mathrm{CDCl}_{3}, 55^{\circ} \mathrm{C}, \delta\right): 6.02-5.94(\mathrm{~m}, 2 \mathrm{H}), 5.20(\mathrm{dd}, \mathrm{J}=10.1,1.4 \mathrm{~Hz}, 1 \mathrm{H}), 5.19-5.13$ $(\mathrm{m}, 1 \mathrm{H}), 3.47-3.30(\mathrm{~m}, 1 \mathrm{H}), 3.22-3.00(\mathrm{~m}, 1 \mathrm{H}), 2.67-2.46(\mathrm{~m}, 1 \mathrm{H}), 2.39-2.34(\mathrm{~m}, 1 \mathrm{H}), 2.30(\mathrm{dd}, \mathrm{J}=$ 13.7, 7.0 Hz, 1H), $2.20(\mathrm{dd}, J=13.7,7.3 \mathrm{~Hz}, 1 \mathrm{H}), 2.18-2.05(\mathrm{~m}, 2 \mathrm{H}), 2.02-1.76(\mathrm{~m}, 9 \mathrm{H}), 1.68-1.48$ $(\mathrm{m}, 9 \mathrm{H}), 1.44-1.16(\mathrm{~m}, 9 \mathrm{H}), 0.91(\mathrm{~s}, 3 \mathrm{H}), 0.88-0.76(\mathrm{~m}, 1 \mathrm{H})$.

${ }^{13} \mathrm{C}$ NMR $\left(151 \mathrm{MHz}, \mathrm{CDCl}_{3}, 55{ }^{\circ} \mathrm{C}, \delta\right): 139.9,135.0,134.9,133.9,123.1,120.4,119.4,119.4,82.5,82.4$, 59.2, 58.0, 56.2, 55.3, 49.9, 49.5, 46.7, 46.6, 46.5, 46.4, 44.1, 42.0, 41.9, 39.0, 36.6, 35.1, 33.8, 32.3, $32.2,31.7,30.2,30.1,29.9,29.4,29.2,29.1,27.4,26.7,26.5,26.0,25.3,25.3,25.0,25.0,25.0,24.9$, $24.5,23.7,23.3,18.0,14.7,14.5$.

HRMS-ESI(m/z) calc'd for $\mathrm{C}_{27} \mathrm{H}_{44} \mathrm{NO}[\mathrm{M}+1]^{+}$, 398.3422; found, 398.3417; deviation: -1.08 ppm.

\section{Epiandrosterone-derivative 30}
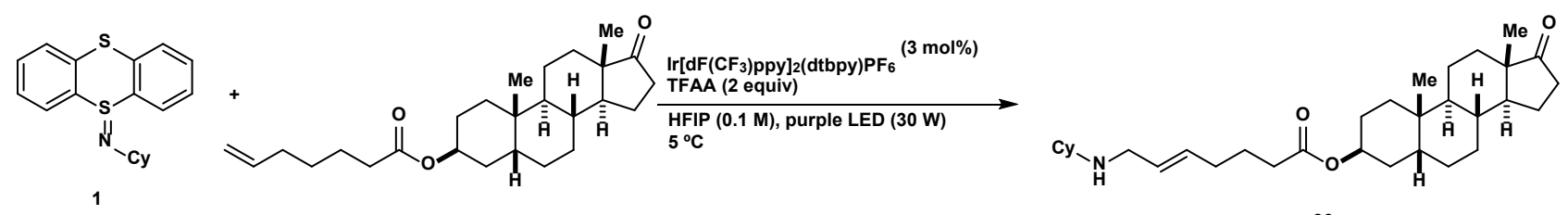

30

Under nitrogen atmosphere, to a 4-mL borosilicate vial equipped with a magnetic stir bar were added thianthrenylidene cyclohexylamine 1 (31.3 $\mathrm{mg}, 0.100 \mathrm{mmol}, 1.00$ equiv.), epiandrosterone derived alkene (48.0 mg, $0.120 \mathrm{mmol}, 1.20$ equiv.), $\operatorname{Ir}\left[\mathrm{dF}\left(\mathrm{CF}_{3}\right) \mathrm{ppy}\right]_{2}\left(\mathrm{dtbpy}_{\mathrm{P}} \mathrm{PF}_{6}(3.3 \mathrm{mg}, 3.0 \mu \mathrm{mol}, 3.0 \mathrm{~mol} \%), \mathrm{HFIP}(1 \mathrm{~mL}, \mathrm{c}=\right.$ $0.1 \mathrm{M})$, and trifluoroacetic anhydride $(28 \mu \mathrm{L}, 42 \mathrm{mg}, 0.20 \mathrm{mmol}, 2.0$ equiv. $)$. The vial was sealed with a septum-cap and irradiated for $3 \mathrm{~h}$ at $5{ }^{\circ} \mathrm{C}$ using a purple LED $(30 \mathrm{~W})$. Then, the reaction mixture was concentrated. The residue was dissolved in DCM $(5 \mathrm{~mL})$ and washed with saturated sodium carbonate aqueous solution $(5 \mathrm{~mL})$. The aqueous phase was extracted with DCM $(2 \times 5 \mathrm{~mL})$. The organic phase was dried over $\mathrm{Na}_{2} \mathrm{SO}_{4}$ and the solvent was removed under reduced pressure. The residue was purified by chromatography on silica gel eluting with $\mathrm{CHCl}_{3} / \mathrm{MeOH}(50 / 1-30 / 1(\mathrm{v} / \mathrm{v}))$ to afford $30.3 \mathrm{mg}$ of $\mathbf{3 0}$ as a colorless oil $(61 \%$ yield, $E: Z=7: 1)$.

$\boldsymbol{R}_{\boldsymbol{f}}=0.30\left(\mathrm{CHCl}_{3} / \mathrm{MeOH}=15 / 1(\mathrm{v} / \mathrm{v})\right)$.

\section{NMR Spectroscopy:}

${ }^{1} \mathrm{H}$ NMR $\left(500 \mathrm{MHz}, \mathrm{CDCl}_{3}, 25^{\circ} \mathrm{C}, \delta\right): 5.80$ (dt, $\left.J=15.5,6.5 \mathrm{~Hz} 1 \mathrm{H}\right), 5.70(\mathrm{dd}, J=15.5,6.5 \mathrm{~Hz}, 1 \mathrm{H}), 4.68$ 
(td, $J=11.1,5.5 \mathrm{~Hz}, 1 \mathrm{H}), 3.50(\mathrm{~d}, J=6.7 \mathrm{~Hz}, 2 \mathrm{H}), 2.87(\mathrm{t}, J=11.3 \mathrm{~Hz}, 1 \mathrm{H}), 2.42(\mathrm{dd}, J=19.3,8.8 \mathrm{~Hz}$, $1 \mathrm{H}), 2.31(\mathrm{~s}, 1 \mathrm{H}), 2.24(\mathrm{t}, J=7.5 \mathrm{~Hz}, 2 \mathrm{H}), 2.16-2.05(\mathrm{~m}, 4 \mathrm{H}), 1.96-1.88(\mathrm{~m}, 1 \mathrm{H}), 1.86-1.48(\mathrm{~m}$, $18 \mathrm{H}), 1.35-1.18(\mathrm{~m}, 10 \mathrm{H}), 1.08-0.92(\mathrm{~m}, 2 \mathrm{H}), 0.84(\mathrm{~s}, 3 \mathrm{H}), 0.84(\mathrm{~s}, 3 \mathrm{H}), 0.70(\mathrm{td}, J=11.4,3.7 \mathrm{~Hz}$, $1 \mathrm{H})$.

${ }^{13} \mathrm{C}$ NMR $\left(126 \mathrm{MHz}, \mathrm{CDCl}_{3}, 25^{\circ} \mathrm{C}, \delta\right): 221.3,172.9,138.6,121.6,73.6,55.2,54.4,51.5,47.9,45.9$, $44.8,36.8,36.0,35.8,35.1,34.1$, 34.1, 31.8, 31.6, 30.9, 29.6, 28.4, 27.6, 25.1, 24.7, 24.3, 21.9, 20.6, 13.9, 12.3.

HRMS-ESI(m/z) calc'd for $\mathrm{C}_{32} \mathrm{H}_{52} \mathrm{NO}_{3}[\mathrm{M}+1]^{+}, 498.3948$; found, 498.3942; deviation: -1.29 ppm.

(Z)-N-(tert-Butyl)-3-phenyl-2-propenamine 31

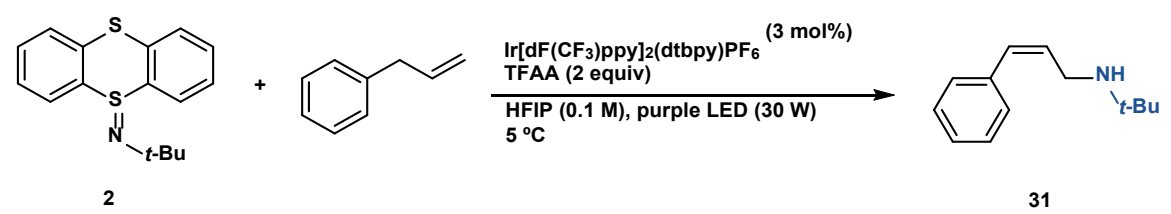

Under nitrogen atmosphere, to a 4-mL borosilicate vial equipped with a magnetic stir bar were added thianthrenylidene tert-butylamine $2(28.7 \mathrm{mg}, 0.100 \mathrm{mmol}, 1.00$ equiv.), allylbenzene (20.0 $\mu \mathrm{L}, 17.7 \mathrm{mg}, 0.150$ mmol, 1.50 equiv.), Ir[dF(CF $)$ ppy $]_{2}\left(\mathrm{dtbpy}_{\mathrm{P}} \mathrm{PF}_{6}(3.3 \mathrm{mg}, 3.0 \mu \mathrm{mol}, 3.0 \mathrm{~mol} \%), \mathrm{HFIP}(1 \mathrm{~mL}, \mathrm{c}=0.1 \mathrm{M})\right.$, and trifluoroacetic anhydride ( $28 \mu \mathrm{L}, 42 \mathrm{mg}, 0.20 \mathrm{mmol}, 2.0$ equiv.). The vial was sealed with a septum-cap and irradiated for $3 \mathrm{~h}$ at $5{ }^{\circ} \mathrm{C}$ using a purple LED $(30 \mathrm{~W})$. Then, the reaction mixture was concentrated. The residue was dissolved in $\mathrm{DCM}(5 \mathrm{~mL})$ and washed with saturated sodium carbonate aqueous solution $(5 \mathrm{~mL})$. The aqueous phase was extracted with DCM $(2 \times 5 \mathrm{~mL})$. The organic phase was dried over $\mathrm{Na}_{2} \mathrm{SO}_{4}$ and the solvent was removed under reduced pressure. The residue was purified by chromatography on silica gel eluting with $\mathrm{CHCl}_{3} / \mathrm{MeOH}(50 / 1-30 / 1(\mathrm{v} / \mathrm{v}))$ to afford $17.2 \mathrm{mg}$ of $\mathbf{3 1}$ as a colorless oil (91\% yield, $\left.\mathrm{E}: \mathrm{Z}=1: 6\right)$.

$\mathbf{R}_{\boldsymbol{f}}=0.25\left(\mathrm{CHCl}_{3} / \mathrm{MeOH}=15 / 1(\mathrm{v} / \mathrm{v})\right)$.

\section{NMR Spectroscopy:}

${ }^{1} \mathrm{H}$ NMR $\left(500 \mathrm{MHz}, \mathrm{CDCl}_{3}, 25^{\circ} \mathrm{C}, \delta\right): 7.36(\mathrm{t}, J=7.6 \mathrm{~Hz}, 2 \mathrm{H}), 7.28(\mathrm{t}, J=7.0 \mathrm{~Hz}, 1 \mathrm{H}), 7.20(\mathrm{~d}, J=7.1$ $\mathrm{Hz}, 2 \mathrm{H}), 6.63(\mathrm{~d}, J=11.4 \mathrm{~Hz}, 1 \mathrm{H}), 5.92(\mathrm{dt}, J=11.6,6.8 \mathrm{~Hz}, 1 \mathrm{H}), 3.76(\mathrm{dd}, J=6.8,1.8 \mathrm{~Hz}, 2 \mathrm{H}), 1.35$ (s, $9 \mathrm{H})$.

${ }^{13} \mathrm{C}$ NMR $\left(126 \mathrm{MHz}, \mathrm{CDCl}_{3}, 25^{\circ} \mathrm{C}, \delta\right): 135.6,134.9,128.7,128.6,127.9,122.2,57.1,39.9,26.0$.

HRMS-ESI(m/z) calc'd for $\mathrm{C}_{13} \mathrm{H}_{20} \mathrm{~N}[\mathrm{M}+1]^{+}, 190.1590$; found, 190.1590; deviation: $0.07 \mathrm{ppm}$.

(Z)-N-(tert-Butyl)-2-cyclooctenamine 32

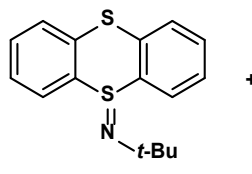

2

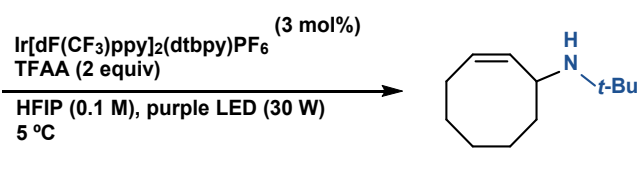

32 
Under nitrogen atmosphere, to a 4-mL borosilicate vial equipped with a magnetic stir bar were added thianthrenylidene tert-butylamine 2 (28.7 mg, 0.100 mmol, 1.00 equiv.), cis-cyclooctene (20.0 $\mu \mathrm{L}, 16.5 \mathrm{mg}$, 0.150 mmol, 1.50 equiv.), $\operatorname{Ir}\left[\mathrm{dF}\left(\mathrm{CF}_{3}\right) \mathrm{ppy}_{2}(\mathrm{dtbpy}) \mathrm{PF}_{6}(3.3 \mathrm{mg}, 3.0 \mu \mathrm{mol}, 3.0 \mathrm{~mol} \%), \mathrm{HFIP}(1 \mathrm{~mL}, \mathrm{c}=0.1 \mathrm{M})\right.$, and trifluoroacetic anhydride ( $28 \mu \mathrm{L}, 42 \mathrm{mg}, 0.20 \mathrm{mmol}, 2.0$ equiv.). The vial was sealed with a septum-cap and irradiated for $3 \mathrm{~h}$ at $5{ }^{\circ} \mathrm{C}$ using a purple LED $(30 \mathrm{~W})$. Then, the reaction mixture was concentrated. The residue was dissolved in DCM $(5 \mathrm{~mL})$ and washed with saturated sodium carbonate aqueous solution $(5 \mathrm{~mL})$. The aqueous phase was extracted with DCM $(2 \times 5 \mathrm{~mL})$. The organic phase was dried over $\mathrm{Na}_{2} \mathrm{SO}_{4}$ and the solvent was removed under reduced pressure. The residue was purified by chromatography on silica gel eluting with $\mathrm{CHCl}_{3} / \mathrm{MeOH}(50 / 1-30 / 1(\mathrm{v} / \mathrm{v}))$ to afford $13.0 \mathrm{mg}$ of 32 as a colorless oil $(72 \%$ yield, r.r. > 20:1).

$\boldsymbol{R}_{\boldsymbol{f}}=0.25\left(\mathrm{CHCl}_{3} / \mathrm{MeOH}=10 / 1(\mathrm{v} / \mathrm{v})\right)$.

\section{NMR Spectroscopy:}

${ }^{1} \mathrm{H}$ NMR $\left(500 \mathrm{MHz}, \mathrm{CDCl}_{3}, 25^{\circ} \mathrm{C}, \delta\right): 5.93(\mathrm{dd}, J=9.6,9.5 \mathrm{~Hz}, 1 \mathrm{H}), 5.80(\mathrm{dt}, J=10.3,9.0 \mathrm{~Hz}, 1 \mathrm{H}), 4.16$ (ddd, $J=12.8,8.5,4.6 \mathrm{~Hz}, 1 \mathrm{H}), 2.48-2.35(\mathrm{~m}, 1 \mathrm{H}), 2.22-2.13(\mathrm{~m}, 1 \mathrm{H}), 2.08-1.97(\mathrm{~m}, 1 \mathrm{H}), 1.92-$ $1.79(\mathrm{~m}, 1 \mathrm{H}), 1.78-1.69(\mathrm{~m}, 2 \mathrm{H}), 1.66-1.58(\mathrm{~m}, 1 \mathrm{H}), 1.48(\mathrm{~s}, 9 \mathrm{H}), 1.46-1.37(\mathrm{~m}, 1 \mathrm{H}), 1.32-1.27(\mathrm{~m}$, $1 \mathrm{H})$.

${ }^{13} \mathrm{C}$ NMR $\left(126 \mathrm{MHz}, \mathrm{CDCl}_{3}, 25^{\circ} \mathrm{C}, \delta\right): 131.4,128.7,58.5,52.6,34.8,29.4,27.3,27.2,26.5,24.1$.

HRMS-EI(m/z) calc'd for $\mathrm{C}_{12} \mathrm{H}_{23} \mathrm{~N}$ [M] ${ }^{+}, 181.1825$; found, 181.1825; deviation: $0.05 \mathrm{ppm}$.

(Z)-N-Propyl-2-cyclooctenamine 33

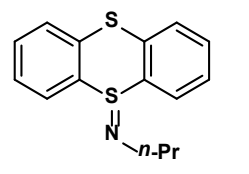

3

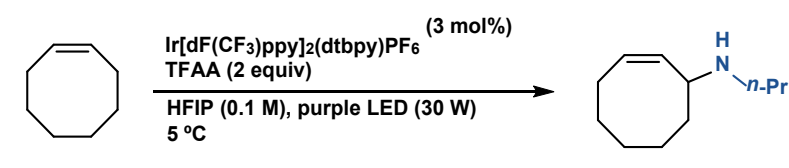

33

Under nitrogen atmosphere, to a 4-mL borosilicate vial equipped with a magnetic stir bar were added thianthrenylidene $n$-propylamine $3(27.3 \mathrm{mg}, 0.100 \mathrm{mmol}, 1.00$ equiv.), cis-cyclooctene $(20.0 \mu \mathrm{L}, 16.5 \mathrm{mg}$, 0.150 mmol, 1.50 equiv.), $\operatorname{Ir}\left[\mathrm{dF}\left(\mathrm{CF}_{3}\right) \mathrm{ppy}_{2}\right.$ (dtbpy)PF 6 (3.3 mg, $\left.3.0 \mu \mathrm{mol}, 3.0 \mathrm{~mol} \%\right), \operatorname{HFIP}(1 \mathrm{~mL}, \mathrm{c}=0.1 \mathrm{M})$, and trifluoroacetic anhydride ( $28 \mu \mathrm{L}, 42 \mathrm{mg}, 0.20 \mathrm{mmol}, 2.0$ equiv.). The vial was sealed with a septum-cap and irradiated for $3 \mathrm{~h}$ at $5{ }^{\circ} \mathrm{C}$ using a purple LED $(30 \mathrm{~W})$. Then, the reaction mixture was concentrated. The residue was dissolved in DCM ( $5 \mathrm{~mL}$ ) and washed with saturated sodium carbonate aqueous solution (5 mL). The aqueous phase was extracted with DCM $(2 \times 5 \mathrm{~mL})$. The organic phase was dried over $\mathrm{Na}_{2} \mathrm{SO}_{4}$ and the solvent was removed under reduced pressure. The residue was purified by chromatography on silica gel eluting with $\mathrm{CHCl}_{3} / \mathrm{MeOH}(50 / 1-30 / 1(\mathrm{v} / \mathrm{v}))$ to afford $13.4 \mathrm{mg}$ of 33 as a colorless oil $(80 \%$ yield, r.r. $=12: 1)$.

$\mathbf{R}_{\boldsymbol{f}}=0.25\left(\mathrm{CHCl}_{3} / \mathrm{MeOH}=10 / 1(\mathrm{v} / \mathrm{v})\right)$.

\section{NMR Spectroscopy:}

${ }^{1} \mathrm{H}$ NMR $\left(500 \mathrm{MHz}, \mathrm{CDCl}_{3}, 25^{\circ} \mathrm{C}, \delta\right): 5.95$ (dtd, $\left.J=9.5,9.0,1.3 \mathrm{~Hz}, 1 \mathrm{H}\right), 5.69(\mathrm{dd}, J=9.6,9.5 \mathrm{~Hz}, 1 \mathrm{H})$, 
$4.09-3.92(\mathrm{~m}, 1 \mathrm{H}), 3.03-2.87(\mathrm{~m}, 1 \mathrm{H}), 2.83-2.73(\mathrm{~m}, 1 \mathrm{H}), 2.41-2.27(\mathrm{~m}, 1 \mathrm{H}), 2.21-2.11(\mathrm{~m}, 1 \mathrm{H})$, $2.09-2.02(\mathrm{~m}, 1 \mathrm{H}), 1.98-1.82(\mathrm{~m}, 3 \mathrm{H}), 1.72-1.64(\mathrm{~m}, 2 \mathrm{H}), 1.64-1.55(\mathrm{~m}, 1 \mathrm{H}), 1.55-1.47(\mathrm{~m}, 1 \mathrm{H})$, $1.41-1.29(\mathrm{~m}, 2 \mathrm{H}), 0.96(\mathrm{t}, J=7.4 \mathrm{~Hz}, 3 \mathrm{H})$.

${ }^{13} \mathrm{C}$ NMR $\left(126 \mathrm{MHz}, \mathrm{CDCl}_{3}, 25^{\circ} \mathrm{C}, \delta\right): 134.9,125.4,56.6,48.2,33.3,29.1,26.8,26.1,24.2,19.6,11.5$. HRMS-ESI(m/z) calc'd for $\mathrm{C}_{11} \mathrm{H}_{22} \mathrm{~N}[\mathrm{M}+1]^{+}, 168.1748$; found, 168.1747; deviation: $-0.81 \mathrm{ppm}$.

(Z)-N-Neopentyl-2-cyclooctenamine 34

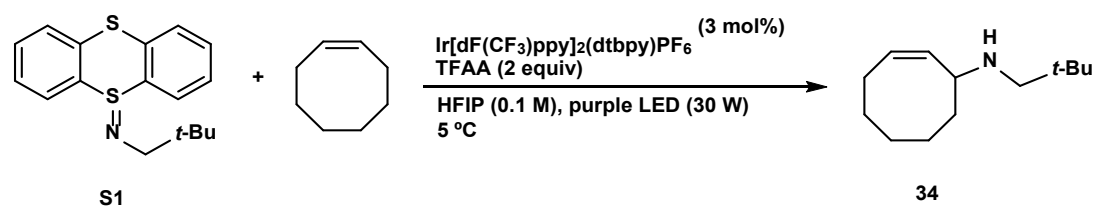

Under nitrogen atmosphere, to a 4-mL borosilicate vial equipped with a magnetic stir bar were added thianthrenylidene neopentylamine $\mathbf{S} 1$ (30.1 mg, $0.100 \mathrm{mmol}, 1.00$ equiv.), cis-cyclooctene $(20.0 \mu \mathrm{L}, 16.5 \mathrm{mg}$, $0.150 \mathrm{mmol}, 1.50$ equiv.), $\operatorname{Ir}\left[\mathrm{dF}\left(\mathrm{CF}_{3}\right) \mathrm{ppy}_{2}(\mathrm{dtbpy}) \mathrm{PF}_{6}(3.3 \mathrm{mg}, 3.0 \mu \mathrm{mol}, 3.0 \mathrm{~mol} \%\right.$ ), HFIP (1 mL, c = $0.1 \mathrm{M}$ ), and trifluoroacetic anhydride ( $28 \mu \mathrm{L}, 42 \mathrm{mg}, 0.20 \mathrm{mmol}, 2.0$ equiv.). The vial was sealed with a septum-cap and irradiated for $3 \mathrm{~h}$ at $5{ }^{\circ} \mathrm{C}$ using a purple LED $(30 \mathrm{~W})$. Then, the reaction mixture was concentrated. The residue was dissolved in $\mathrm{DCM}(5 \mathrm{~mL})$ and washed with saturated sodium carbonate aqueous solution $(5 \mathrm{~mL})$. The aqueous phase was extracted with DCM $(2 \times 5 \mathrm{~mL})$. The organic phase was dried over $\mathrm{Na}_{2} \mathrm{SO}_{4}$ and the solvent was removed under reduced pressure. The residue was purified by chromatography on silica gel eluting with $\mathrm{CHCl}_{3} / \mathrm{MeOH}(50 / 1-30 / 1(\mathrm{v} / \mathrm{v}))$ to afford $16.6 \mathrm{mg}$ of 34 as a colorless oil $(85 \%$ yield, r.r. $=10: 1)$.

$\mathbf{R} \boldsymbol{f}=0.25\left(\mathrm{CHCl}_{3} / \mathrm{MeOH}=10 / 1(\mathrm{v} / \mathrm{v})\right)$.

\section{NMR Spectroscopy:}

${ }^{1} \mathrm{H}$ NMR $\left(500 \mathrm{MHz}, \mathrm{CDCl}_{3}, 25{ }^{\circ} \mathrm{C}, \delta\right): 5.65$ (dddd, $J=10.6,8.8,7.2,1.5 \mathrm{~Hz}, 1 \mathrm{H}$ ), 5.34 (ddd, $J=10.7,7.7$, $1.4 \mathrm{~Hz}, 1 \mathrm{H}$ ), 3.49 (dddd, $J=11.8,7.8,4.3,1.4 \mathrm{~Hz}, 1 \mathrm{H}), 2.37$ (d, $J=11.3 \mathrm{~Hz}, 1 \mathrm{H}), 2.30$ (d, J = 11.3 Hz, $1 \mathrm{H}), 2.27-2.15(\mathrm{~m}, 1 \mathrm{H}), 2.10-2.02(\mathrm{~m}, 1 \mathrm{H}), 1.75(\mathrm{td}, J=8.8,4.5 \mathrm{~Hz}, 1 \mathrm{H}), 1.69-1.60(\mathrm{~m}, 2 \mathrm{H}), 1.58-$ $1.49(\mathrm{~m}, 1 \mathrm{H}), 1.38-1.26(\mathrm{~m}, 2 \mathrm{H}), 0.90(\mathrm{~s}, 9 \mathrm{H})$.

${ }^{13} \mathrm{C}$ NMR $\left(126 \mathrm{MHz} \mathrm{CDCl}_{3}, 2{ }^{\circ} \mathrm{C}, \delta\right): 136.4,129.6,60.8,56.5,37.1,31.4,29.6,28.0,26.9,26.8,24.9$.

HRMS-ESI(m/z) calc'd for $\mathrm{C}_{13} \mathrm{H}_{25} \mathrm{~N}[\mathrm{M}+1]^{+}, 195.1981$; found, 195.1981; deviation: -0.01 ppm.

(Z)-N-Adamantyl-2-cyclooctenamine 35

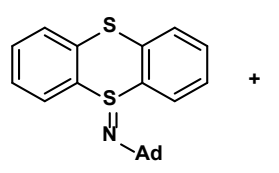

4

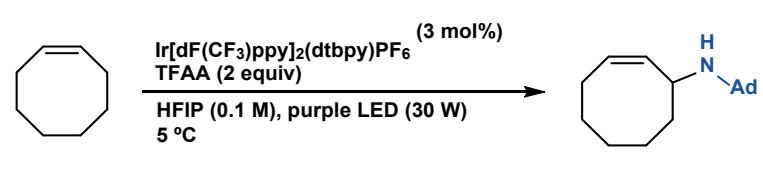

35

Under nitrogen atmosphere, to a 4-mL borosilicate vial equipped with a magnetic stir bar were added thianthrenylidene 1-adamantanamine 4 (36.5 mg, 0.100 mmol, 1.00 equiv.), cis-cyclooctene (20.0 $\mu \mathrm{L}, 16.5$ 
mg, 0.150 mmol, 1.50 equiv.), Ir[dF(CF3)ppy 2 (dtbpy)PF $(3.3 \mathrm{mg}, 3.0 \mu \mathrm{mol}, 3.0 \mathrm{~mol} \%), \mathrm{HFIP}(1 \mathrm{~mL}, \mathrm{c}=0.1$ $\mathrm{M})$, and trifluoroacetic anhydride ( $28 \mu \mathrm{L}, 42 \mathrm{mg}, 0.20 \mathrm{mmol}, 2.0$ equiv.). The vial was sealed with a septumcap and irradiated for $3 \mathrm{~h}$ at $5{ }^{\circ} \mathrm{C}$ using a purple LED $(30 \mathrm{~W})$. Then, the reaction mixture was concentrated. The residue was dissolved in DCM $(5 \mathrm{~mL})$ and washed with saturated sodium carbonate aqueous solution (5 $\mathrm{mL})$. The aqueous phase was extracted with $\mathrm{DCM}(2 \times 5 \mathrm{~mL})$. The organic phase was dried over $\mathrm{Na}_{2} \mathrm{SO}_{4}$ and the solvent was removed under reduced pressure. The residue was purified by chromatography on silica gel eluting with $\mathrm{CHCl}_{3} / \mathrm{MeOH}(50 / 1-30 / 1(\mathrm{v} / \mathrm{v}))$ to afford $18.4 \mathrm{mg}$ of 35 as a colorless oil $(71 \%$ yield, r.r. > 20:1).

$\mathbf{R}_{\boldsymbol{f}}=0.25\left(\mathrm{CHCl}_{3} / \mathrm{MeOH}=15 / 1(\mathrm{v} / \mathrm{v})\right)$.

\section{NMR Spectroscopy:}

${ }^{1} \mathrm{H}$ NMR $\left(500 \mathrm{MHz}, \mathrm{CDCl}_{3}, 25{ }^{\circ} \mathrm{C}, \delta\right): 5.93(\mathrm{dd}, J=9.6,9.5 \mathrm{~Hz}, 1 \mathrm{H}), 5.75(\mathrm{dt}, J=9.5,9.0 \mathrm{~Hz}, 1 \mathrm{H}), 4.30-$ $4.09(\mathrm{~m}, 1 \mathrm{H}), 3.81-3.57(\mathrm{~m}, 1 \mathrm{H}), 2.47-2.30(\mathrm{~m}, 1 \mathrm{H}), 2.23-1.99(\mathrm{~m}, 10 \mathrm{H}), 1.93-1.81(\mathrm{~m}, 2 \mathrm{H}), 1.78-$ $1.58(\mathrm{~m}, 9 \mathrm{H}), 1.52-1.39(\mathrm{~m}, 1 \mathrm{H}), 1.37-1.16(\mathrm{~m}, 1 \mathrm{H})$.

${ }^{13} \mathrm{C}$ NMR $\left(126 \mathrm{MHz} \mathrm{CDCl}_{3}, 2{ }^{\circ} \mathrm{C}, \delta\right): 130.5,129.7,68.1,58.8,50.5,39.9,35.8,35.0,29.4,27.3,26.6$, $25.7,24.1$.

HRMS-ESI(m/z) calc'd for $\mathrm{C}_{18} \mathrm{H}_{30} \mathrm{~N}$ [M+1] $]^{+}, 260.2374$; found, 260.2373; deviation: -0.49 ppm.

(Z)-2-(2-Cyclooctenylamino)ethyl pivalate 36
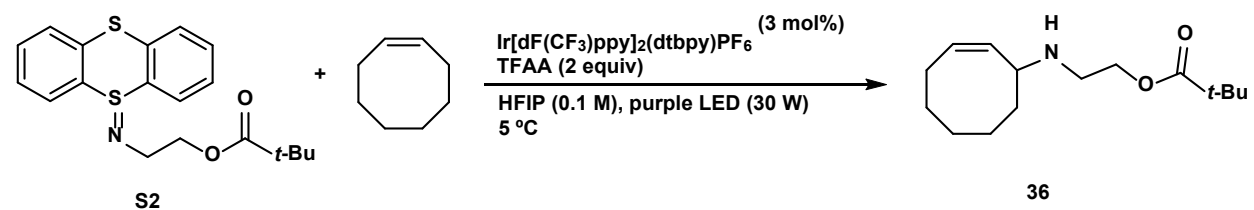

36

Under nitrogen atmosphere, to a 4-mL borosilicate vial equipped with a magnetic stir bar were added thianthrenylidene-2-aminoethyl pivalate $\mathbf{S} 2(35.9 \mathrm{mg}, 0.100 \mathrm{mmol}, 1.00$ equiv.), cis-cyclooctene $(20.0 \mu \mathrm{L}$, $16.5 \mathrm{mg}, 0.150 \mathrm{mmol}, 1.50$ equiv.), $\operatorname{Ir}\left[\mathrm{dF}\left(\mathrm{CF}_{3}\right) \text { ppy }\right]_{2}\left(\mathrm{dtbpy}_{\mathrm{P}} \mathrm{PF}_{6}(3.3 \mathrm{mg}, 3.0 \mu \mathrm{mol}, 3.0 \mathrm{~mol} \%)\right.$, HFIP $(1 \mathrm{~mL}, \mathrm{c}=$ $0.1 \mathrm{M})$, and trifluoroacetic anhydride $(28 \mu \mathrm{L}, 42 \mathrm{mg}, 0.20 \mathrm{mmol}, 2.0$ equiv.). The vial was sealed with a septum-cap and irradiated for $3 \mathrm{~h}$ at $5{ }^{\circ} \mathrm{C}$ using a purple LED $(30 \mathrm{~W})$. Then, the reaction mixture was concentrated. The residue was dissolved in DCM $(5 \mathrm{~mL})$ and washed with saturated sodium carbonate aqueous solution $(5 \mathrm{~mL})$. The aqueous phase was extracted with DCM $(2 \times 5 \mathrm{~mL})$. The organic phase was dried over $\mathrm{Na}_{2} \mathrm{SO}_{4}$ and the solvent was removed under reduced pressure. The residue was purified by chromatography on silica gel eluting with $\mathrm{CHCl}_{3} / \mathrm{MeOH}(50 / 1-30 / 1(\mathrm{v} / \mathrm{v}))$ to afford $15.5 \mathrm{mg}$ of 36 as a colorless oil $(61 \%$ yield, r.r. $=12: 1)$.

$\mathbf{R}_{\boldsymbol{f}}=0.25\left(\mathrm{CHCl}_{3} / \mathrm{MeOH}=15 / 1(\mathrm{v} / \mathrm{v})\right)$.

\section{NMR Spectroscopy:}

${ }^{1} \mathrm{H}$ NMR $\left(600 \mathrm{MHz}, \mathrm{CDCl}_{3}, 25^{\circ} \mathrm{C}, \delta\right): 5.77$ (dt, $\left.J=8.0,7.5 \mathrm{~Hz}, 1 \mathrm{H}\right), 5.38(\mathrm{dd}, J=8.0,7.0 \mathrm{~Hz}, 1 \mathrm{H}), 4.29-$ $4.17(\mathrm{~m}, 2 \mathrm{H}), 3.77-3.68(\mathrm{~m}, 1 \mathrm{H}), 3.04-2.88(\mathrm{~m}, 2 \mathrm{H}), 2.23-2.06(\mathrm{~m}, 1 \mathrm{H}), 1.93-1.78(\mathrm{~m}, 1 \mathrm{H}), 1.73-$ 
$1.62(\mathrm{~m}, 2 \mathrm{H}), 1.59-1.51(\mathrm{~m}, 2 \mathrm{H}), 1.48-1.39(\mathrm{~m}, 2 \mathrm{H}), 1.36-1.27(\mathrm{~m}, 1 \mathrm{H}), 1.22(\mathrm{~s}, 9 \mathrm{H})$.

${ }^{13} \mathrm{C}$ NMR $\left(151 \mathrm{MHz}, \mathrm{CDCl}_{3}, 2{ }^{\circ} \mathrm{C}, \delta\right): 178.7,131.9,129.9,63.3,55.6,46.2,39.0,36.4,29.4,27.4,27.0$, 26.6, 24.7.

HRMS-ESI(m/z) calc'd for $\mathrm{C}_{15} \mathrm{H}_{28} \mathrm{NO}_{2}[\mathrm{M}+1]^{+}, 254.2114$; found, 254.2115; deviation: 0.09 ppm.

(Z)-3-(Cyclooct-2-en-1-ylamino)propan-1-ol 37

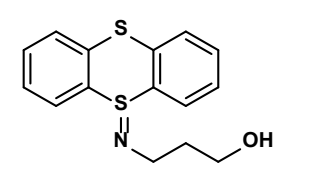

S5

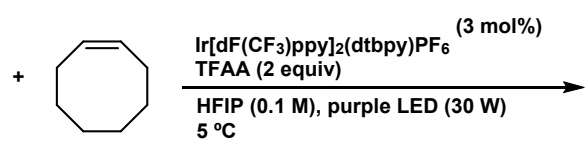

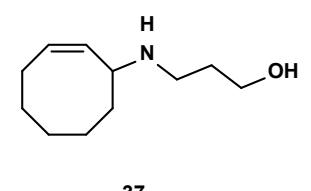

37

Under nitrogen atmosphere, to a 4-mL borosilicate vial equipped with a magnetic stir bar were added 3(thianthrenylidene)aminopropan-1-ol S5 (28.9 mg, $0.100 \mathrm{mmol}, 1.00$ equiv.), cis-cyclooctene (20.0 $\mu \mathrm{L}, 16.5$ $\mathrm{mg}, 0.150 \mathrm{mmol}, 1.50$ equiv.), $\operatorname{Ir}\left[\mathrm{dF}\left(\mathrm{CF}_{3}\right) \mathrm{ppy}\right]_{2}(\mathrm{dtbpy}) \mathrm{PF} 6(3.3 \mathrm{mg}, 3.0 \mu \mathrm{mol}, 3.0 \mathrm{~mol} \%), \mathrm{HFIP}(1 \mathrm{~mL}, \mathrm{c}=0.1$ $\mathrm{M})$, and trifluoroacetic anhydride ( $28 \mu \mathrm{L}, 42 \mathrm{mg}, 0.20 \mathrm{mmol}, 2.0$ equiv.). The vial was sealed with a septumcap and irradiated for $3 \mathrm{~h}$ at $5{ }^{\circ} \mathrm{C}$ using a purple LED $(30 \mathrm{~W})$. Then, the reaction mixture was concentrated. The residue was dissolved in DCM $(5 \mathrm{~mL})$ and washed with saturated sodium carbonate aqueous solution (5 $\mathrm{mL})$. The aqueous phase was extracted with $\mathrm{DCM}(2 \times 5 \mathrm{~mL})$. The organic phase was dried over $\mathrm{Na}_{2} \mathrm{SO}_{4}$ and the solvent was removed under reduced pressure. The residue was purified by chromatography on silica gel eluting with $\mathrm{CHCl}_{3} / \mathrm{MeOH}(40 / 1-10 / 1(\mathrm{v} / \mathrm{v}))$ to afford $12.2 \mathrm{mg}$ of 37 as a colorless oil $(67 \%$ yield, r.r. $=12: 1)$.

$\boldsymbol{R}_{\boldsymbol{f}}=0.20\left(\mathrm{CHCl}_{3} / \mathrm{MeOH}=10 / 1(\mathrm{v} / \mathrm{v})\right)$.

\section{NMR Spectroscopy:}

${ }^{1} \mathrm{H}$ NMR $\left(600 \mathrm{MHz}, \mathrm{CDCl}_{3}, 25^{\circ} \mathrm{C}, \delta\right): 5.72(\mathrm{dt}, J=10.1,9.5 \mathrm{~Hz}, 1 \mathrm{H}), 5.32(\mathrm{dd}, J=10.8,7.9 \mathrm{~Hz}, 1 \mathrm{H}), 3.80$ (t, $J=5.3 \mathrm{~Hz}, 2 \mathrm{H}), 3.62-3.52(\mathrm{~m}, 1 \mathrm{H}), 3.16(\mathrm{br}, 1 \mathrm{H}), 2.93-2.81(\mathrm{~m}, 2 \mathrm{H}), 2.25-2.11(\mathrm{~m}, 1 \mathrm{H}), 2.11-$ $2.00(\mathrm{~m}, 1 \mathrm{H}), 1.86-1.58(\mathrm{~m}, 5 \mathrm{H}), 1.54-1.48(\mathrm{~m}, 2 \mathrm{H}), 1.44-1.21(\mathrm{~m}, 3 \mathrm{H})$.

${ }^{13} \mathrm{C}$ NMR $\left(151 \mathrm{MHz}, \mathrm{CDCl}_{3}, 25{ }^{\circ} \mathrm{C}, \delta\right): 134.1,131.1,64.2,55.8,47.9,36.8,31.0,29.4,26.9,26.7,24.7$.

HRMS-ESI(m/z) calc'd for $\mathrm{C}_{15} \mathrm{H}_{28} \mathrm{NO}_{2}[\mathrm{M}+1]^{+}, 254.2114$; found, 254.2115; deviation: 0.09 ppm.

Ethyl (E)-2-methyl-2-(4-phenyl-2-butenylamino)propanoate 38

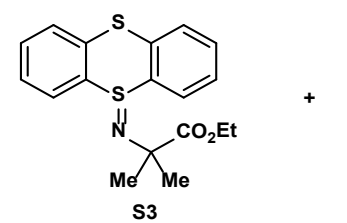

s3

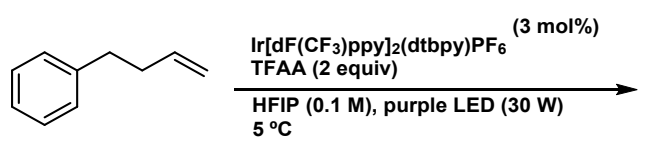

$5^{\circ} \mathrm{C}$

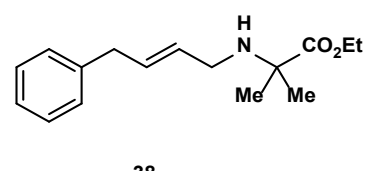

38

Under nitrogen atmosphere, to a 4-mL borosilicate vial equipped with a magnetic stir bar were added ethyl 2(thianthrenylidene amino)-2-methylpropanoate $\mathbf{S} 3 \mathbf{3} 3.5 \mathrm{mg}, 0.100 \mathrm{mmol}, 1.00$ equiv.), 4-phenyl-1-butene (23.0 $\mu \mathrm{L}, 19.8 \mathrm{mg}, 0.150 \mathrm{mmol}, 1.50$ equiv.), Ir[dF(CF $)$ ppy $]_{2}(\mathrm{dtbpy}) \mathrm{PF}_{6}(3.3 \mathrm{mg}, 3.0 \mu \mathrm{mol}, 3.0 \mathrm{~mol} \%)$, HFIP (1 $\mathrm{mL}, \mathrm{c}=0.1 \mathrm{M})$, and trifluoroacetic anhydride $(28 \mu \mathrm{L}, 42 \mathrm{mg}, 0.20 \mathrm{mmol}, 2.0$ equiv.). The vial was sealed with 
a septum-cap and irradiated for $3 \mathrm{~h}$ at $5^{\circ} \mathrm{C}$ using a purple LED $(30 \mathrm{~W})$. Then, the reaction mixture was concentrated. The residue was dissolved in DCM $(5 \mathrm{~mL})$ and washed with saturated sodium carbonate aqueous solution $(5 \mathrm{~mL})$. The aqueous phase was extracted with $\mathrm{DCM}(2 \times 5 \mathrm{~mL})$. The organic phase was dried over $\mathrm{Na}_{2} \mathrm{SO}_{4}$ and the solvent was removed under reduced pressure. The residue was purified by chromatography on silica gel eluting with $\mathrm{CHCl}_{3} / \mathrm{MeOH}(50 / 1-30 / 1(\mathrm{v} / \mathrm{v}))$ to afford $12.5 \mathrm{mg}$ of $\mathbf{3 8}$ as a colorless oil ( $48 \%$ yield, $E: Z=5: 1)$.

$\mathbf{R}_{\boldsymbol{f}}=0.25\left(\mathrm{CHCl}_{3} / \mathrm{MeOH}=10 / 1(\mathrm{v} / \mathrm{v})\right)$.

\section{NMR Spectroscopy:}

${ }^{1} \mathrm{H}$ NMR $\left(500 \mathrm{MHz}, \mathrm{CDCl}_{3}, 25{ }^{\circ} \mathrm{C}, \delta\right): 7.28(\mathrm{t}, J=7.4 \mathrm{~Hz}, 2 \mathrm{H}), 7.21-7.16(\mathrm{~m}, 3 \mathrm{H}), 5.77(\mathrm{dt}, J=15.1,6.7$ $\mathrm{Hz}, 1 \mathrm{H}), 5.59(\mathrm{dt}, J=15.3,6.3 \mathrm{~Hz}, 1 \mathrm{H}), 4.16(\mathrm{q}, J=7.2 \mathrm{~Hz}, 2 \mathrm{H}), 3.35(\mathrm{~d}, J=6.7 \mathrm{~Hz}, 2 \mathrm{H}), 3.10(\mathrm{dd}, J=$ 6.3, $1.3 \mathrm{~Hz}, 2 \mathrm{H}), 1.32(\mathrm{~s}, 6 \mathrm{H}), 1.26(\mathrm{t}, J=7.1 \mathrm{~Hz}, 3 \mathrm{H})$.

${ }^{13} \mathrm{C}$ NMR $\left(126 \mathrm{MHz}, \mathrm{CDCl}_{3}, 25{ }^{\circ} \mathrm{C}, \delta\right): 176.9,140.4,131.8,129.4,128.7,128.5,126.2,60.9,59.1,46.6$, $39.0,25.4,14.4$.

HRMS-ESI(m/z) calc'd for $\mathrm{C}_{16} \mathrm{H}_{24} \mathrm{NO}_{2}[\mathrm{M}+1]^{+}, 262.1803$; found, 262.1802; deviation: -0.44 ppm.

(E)-5-Methyl-N-(1-phenylethyl)-2-hexenamine 39

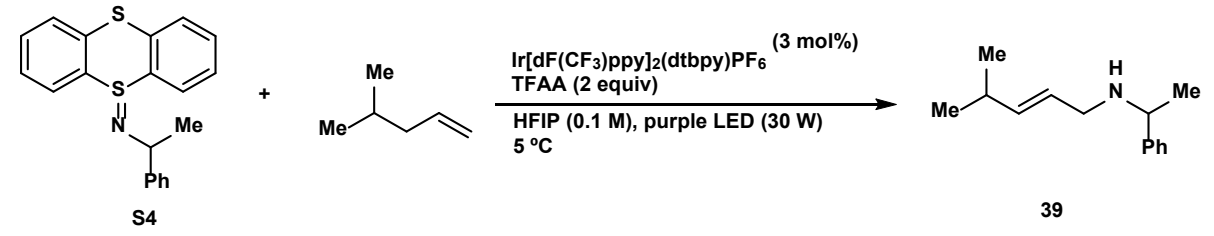

Under nitrogen atmosphere, to a 4-mL borosilicate vial equipped with a magnetic stir bar were added thianthrenylidene 1-phenylethanamine $\mathbf{S} 4(33.5 \mathrm{mg}, 0.100 \mathrm{mmol}, 1.00$ equiv.), 4-methyl-1-pentene $(19.0 \mu \mathrm{L}$, $12.6 \mathrm{mg}, 0.150 \mathrm{mmol}, 1.50$ equiv.), $\operatorname{Ir}\left[\mathrm{dF}\left(\mathrm{CF}_{3}\right) \mathrm{ppy}\right]_{2}(\mathrm{dtbpy}) \mathrm{PF}_{6}(3.3 \mathrm{mg}, 3.0 \mu \mathrm{mol}, 3.0 \mathrm{~mol} \%)$, HFIP $(1 \mathrm{~mL}, \mathrm{c}=$ $0.1 \mathrm{M})$, and trifluoroacetic anhydride $(28 \mu \mathrm{L}, 42 \mathrm{mg}, 0.20 \mathrm{mmol}, 2.0$ equiv.). The vial was sealed with a septum-cap and irradiated for $3 \mathrm{~h}$ at $5{ }^{\circ} \mathrm{C}$ using a purple LED (30 W). Then, the reaction mixture was concentrated. The residue was dissolved in DCM $(5 \mathrm{~mL})$ and washed with saturated sodium carbonate aqueous solution $(5 \mathrm{~mL})$. The aqueous phase was extracted with DCM $(2 \times 5 \mathrm{~mL})$. The organic phase was dried over $\mathrm{Na}_{2} \mathrm{SO}_{4}$ and the solvent was removed under reduced pressure. The residue was purified by chromatography on silica gel eluting with $\mathrm{CHCl}_{3} / \mathrm{MeOH}(50 / 1-30 / 1(\mathrm{v} / \mathrm{v}))$ to afford $16.0 \mathrm{mg}$ of 39 as a colorless oil $(79 \%$ yield, E:Z > 20:1).

$\mathbf{R}_{\boldsymbol{f}}=0.25\left(\mathrm{CHCl}_{3} / \mathrm{MeOH}=15 / 1(\mathrm{v} / \mathrm{v})\right)$.

\section{NMR Spectroscopy:}

${ }^{1} \mathrm{H}$ NMR $\left(500 \mathrm{MHz}, \mathrm{CDCl}_{3}, 25^{\circ} \mathrm{C}, \delta\right): 7.42(\mathrm{~d}, J=7.0 \mathrm{~Hz}, 2 \mathrm{H}), 7.37$ (t, $\left.J=7.6 \mathrm{~Hz}, 2 \mathrm{H}\right), 7.29$ (t, $J=7.3$ $\mathrm{Hz}, 1 \mathrm{H}), 5.61-5.44(\mathrm{~m}, 2 \mathrm{H}), 4.03-3.90(\mathrm{~m}, 1 \mathrm{H}), 3.23(\mathrm{~d}, J=13.1 \mathrm{~Hz}, 1 \mathrm{H}), 3.12-3.02(\mathrm{~m}, 1 \mathrm{H}), 2.34-$ $2.22(\mathrm{~m}, 1 \mathrm{H}), 1.56(\mathrm{~d}, J=6.7 \mathrm{~Hz}, 3 \mathrm{H}), 0.98(\mathrm{~d}, J=6.7 \mathrm{~Hz}, 3 \mathrm{H}), 0.96(\mathrm{~d}, J=6.8 \mathrm{~Hz}, 3 \mathrm{H})$. 
${ }^{13} \mathrm{C}$ NMR $\left(151 \mathrm{MHz}, \mathrm{CDCl}_{3}, 25{ }^{\circ} \mathrm{C}, \delta\right): 129.1,128.7,128.7,128.5,128.4,127.6,126.8,126.0,57.2$, 48.0, 31.1, 29.9, 26.9, 22.3, 22.2.

HRMS-ESI(m/z) calc'd for $\mathrm{C}_{14} \mathrm{H}_{22} \mathrm{~N}[\mathrm{M}+1]^{+}, 204.1745$; found, 204.1747; deviation: 0.80 ppm.

N-(tert-Butyl)-3-phenyl-2-((2,2,6,6-tetramethylpiperidin-1-yl)oxy)propanamine 40

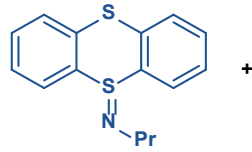

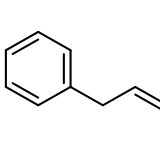

-
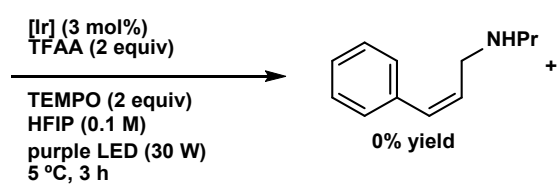

$0 \%$ yield

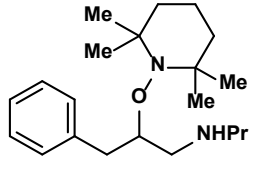

40

Under nitrogen atmosphere, to a 4-mL borosilicate vial equipped with a magnetic stir bar were added thianthrenylidene propylamine $3(27.3 \mathrm{mg}, 0.100 \mathrm{mmol}, 1.00$ equiv.), allylbenzene (20.0 $\mu \mathrm{L}, 17.7 \mathrm{mg}, 0.150$ mmol, 1.50 equiv.), TEMPO (31.2 mg, 0.200 mmol, 2.00 equiv.), $\operatorname{lr}\left[\mathrm{dF}\left(\mathrm{CF}_{3}\right)\right.$ ppy]2(dtbpy)PF6 (3.3 mg, $3.0 \mu \mathrm{mol}$, $3.0 \mathrm{~mol} \%)$, HFIP ( $1 \mathrm{~mL}, \mathrm{c}=0.1 \mathrm{M})$, and trifluoroacetic anhydride ( $28 \mu \mathrm{L}, 42 \mathrm{mg}, 0.20 \mathrm{mmol}, 2.0$ equiv.). The vial was sealed with a septum-cap and irradiated for $3 \mathrm{~h}$ at $5{ }^{\circ} \mathrm{C}$ using a purple LED $(30 \mathrm{~W})$. Then, the reaction mixture was concentrated. The residue was dissolved in DCM $(5 \mathrm{~mL})$ and washed with saturated sodium carbonate aqueous solution $(5 \mathrm{~mL})$. The aqueous phase was extracted with $\mathrm{DCM}(2 \times 5 \mathrm{~mL})$. The organic phase was dried over $\mathrm{Na}_{2} \mathrm{SO}_{4}$ and the solvent was removed under reduced pressure. The residue was purified by chromatography on silica gel eluting with $\mathrm{CH}_{2} \mathrm{Cl}_{2} / \mathrm{MeOH}(50 / 1-30 / 1(\mathrm{v} / \mathrm{v}))$ to afford $5.2 \mathrm{mg}$ of 40 as a colorless oil ( $15 \%$ yield).

$\boldsymbol{R}_{\boldsymbol{f}}=0.20\left(\mathrm{CH}_{2} \mathrm{Cl}_{2} / \mathrm{MeOH}=15 / 1(\mathrm{v} / \mathrm{v})\right)$.

\section{NMR Spectroscopy:}

${ }^{1} \mathrm{H}$ NMR $\left(500 \mathrm{MHz}, \mathrm{CDCl}_{3}, 25^{\circ} \mathrm{C}, \delta\right): 7.32-7.28(\mathrm{~m}, 3 \mathrm{H}), 7.26-7.21(\mathrm{~m}, 2 \mathrm{H}), 4.95-4.80(\mathrm{~m}, 1 \mathrm{H}), 3.33$ (d, $J=12.7 \mathrm{~Hz}, 1 \mathrm{H}), 3.09-2.95(\mathrm{~m}, 2 \mathrm{H}), 2.89(\mathrm{dd}, J=14.0,4.5 \mathrm{~Hz}, 1 \mathrm{H}), 2.79(\mathrm{dd}, J=14.0,6.9 \mathrm{~Hz}, 1 \mathrm{H})$, $2.69(\mathrm{dt}, J=12.0,8.0 \mathrm{~Hz}, 1 \mathrm{H}), 1.96-1.83(\mathrm{~m}, 2 \mathrm{H}), 1.64-1.56(\mathrm{~m}, 2 \mathrm{H}), 1.53-1.46(\mathrm{~m}, 1 \mathrm{H}), 1.41-1.36$ (m, 1H), $1.30(\mathrm{~s}, 3 \mathrm{H}), 1.14(\mathrm{~s}, 3 \mathrm{H}), 1.08(\mathrm{~s}, 3 \mathrm{H}), 1.04(\mathrm{t}, J=7.5 \mathrm{~Hz}, 3 \mathrm{H}), 0.97(\mathrm{~s}, 3 \mathrm{H})$.

${ }^{13} \mathrm{C}$ NMR $\left(151 \mathrm{MHz}, \mathrm{CDCl}_{3}, 25^{\circ} \mathrm{C}, \delta\right): 136.3,129.9,128.5,127.0,75.9,62.6,60.7,53.7,49.4,40.6$, $40.4,38.8,34.8,33.2,21.0,20.6,20.4,17.0,11.6$.

HRMS-ESI(m/z) calc'd for $\mathrm{C}_{21} \mathrm{H}_{37} \mathrm{~N}_{2} \mathrm{O}[\mathrm{M}+1]^{+}$, 333.2900; found, 333.2900; deviation: $0.11 \mathrm{ppm}$.

(E)-N-(5,5-Dichloropenta-2,4-dien-1-yl)cyclohexanamine 41

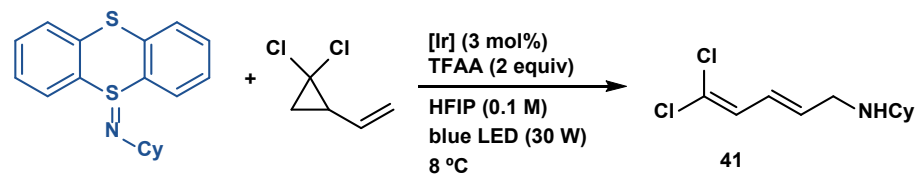

Under nitrogen atmosphere, to a 4-mL borosilicate vial equipped with a magnetic stir bar were added thianthrenylidene cyclohexylamine 1 (27.3 mg, $0.100 \mathrm{mmol}, 1.00$ equiv.), 1,1-dichloro-2-vinylcyclopropane

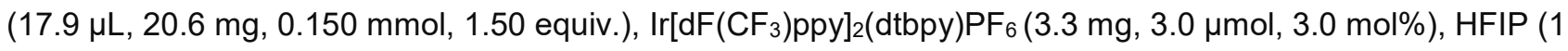


$\mathrm{mL}, \mathrm{c}=0.1 \mathrm{M})$, and trifluoroacetic anhydride $(28 \mu \mathrm{L}, 42 \mathrm{mg}, 0.20 \mathrm{mmol}, 2.0$ equiv.). The vial was sealed with a septum-cap and irradiated for $3 \mathrm{~h}$ at $8{ }^{\circ} \mathrm{C}$ using a blue LED $(30 \mathrm{~W})$. Then, the reaction mixture was concentrated. The residue was dissolved in DCM $(5 \mathrm{~mL})$ and washed with saturated sodium carbonate aqueous solution $(5 \mathrm{~mL})$. The aqueous phase was extracted with DCM $(2 \times 5 \mathrm{~mL})$. The organic phase was dried over $\mathrm{Na}_{2} \mathrm{SO}_{4}$ and the solvent was removed under reduced pressure. The residue was purified by chromatography on silica gel eluting with $\mathrm{CHCl}_{3} / \mathrm{MeOH}(50 / 1-30 / 1(\mathrm{v} / \mathrm{v}))$ to afford $16.7 \mathrm{mg}$ of 41 as a colorless oil $(71 \%$ yield, $E: Z=16: 1)$.

$\mathbf{R}_{\boldsymbol{f}}=0.30\left(\mathrm{CHCl}_{3} / \mathrm{MeOH}=15 / 1(\mathrm{v} / \mathrm{v})\right)$.

\section{NMR Spectroscopy:}

${ }^{1} \mathrm{H}$ NMR $\left(500 \mathrm{MHz}, \mathrm{CDCl}_{3}, 25^{\circ} \mathrm{C}, \delta\right): 6.48(\mathrm{dd}, J=15.4,10.4 \mathrm{~Hz}, 1 \mathrm{H}), 6.39(\mathrm{~d}, J=10.5 \mathrm{~Hz}, 1 \mathrm{H}), 5.90(\mathrm{dt}$, $J=14.8,7.2 \mathrm{~Hz}, 1 \mathrm{H}), 3.59(\mathrm{dd}, J=7.3,1.3 \mathrm{~Hz}, 2 \mathrm{H}), 2.97-2.79(\mathrm{~m}, 1 \mathrm{H}), 2.04(\mathrm{~d}, J=8.8 \mathrm{~Hz}, 2 \mathrm{H}), 1.89-$ $1.77(\mathrm{~m}, 2 \mathrm{H}), 1.67(\mathrm{~d}, J=12.0 \mathrm{~Hz}, 1 \mathrm{H}), 1.39(\mathrm{tt}, J=11.9,6.0 \mathrm{~Hz}, 2 \mathrm{H}), 1.28-1.11(\mathrm{~m}, 4 \mathrm{H})$.

${ }^{13} \mathrm{C}$ NMR $\left(126 \mathrm{MHz}, \mathrm{CDCl}_{3}, 25{ }^{\circ} \mathrm{C}, \delta\right): 131.2,127.3,126.2,124.6,56.4,46.1,29.5,25.1,24.6$.

HRMS-ESI(m/z) calc'd for $\mathrm{C}_{11} \mathrm{H}_{18} \mathrm{NCl}_{2}[\mathrm{M}+1]^{+}, 234.0810$; found, 234.0811; deviation: $0.13 \mathrm{ppm}$.

\section{2-(2-methylprop-1-en-1-yl)hex-5-en-1-yl acetate 42}

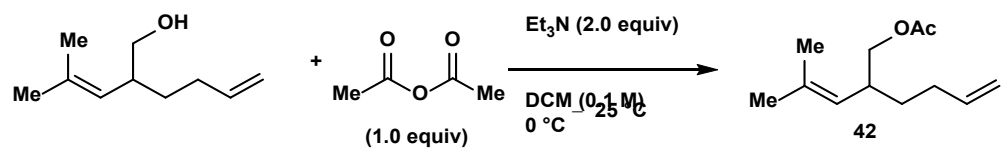

Under air, to a 50-mL flask equipped with a magnetic stir bar were added 2-(2-Methyl-1-propen-1-yl)-5hexen-1-ol (568 $\mu \mathrm{L}, 500 \mathrm{mg}, 3.24 \mathrm{mmol}, 1.00$ equiv.) and DCM (32 mL, c $=0.10 \mathrm{M})$. The flask was sealed with a rubber septum equipped with an argon balloon, and the reaction mixture was chilled in an ice-water cooling bath. Triethylamine ( $903 \mu \mathrm{L}, 656 \mathrm{mg}, 6.48 \mathrm{mmol}, 2.00$ equiv.) was slowly added into the reaction mixture while cooling, and subsequently, acetic anhydride (306 $\mu \mathrm{L}, 331 \mathrm{mg}, 3.24 \mathrm{mmol}, 1.00$ equiv.) was added dropwise within $2 \mathrm{~min}$. After addition, the reaction mixture was stirred at $0{ }^{\circ} \mathrm{C}$ for $30 \mathrm{~min}$, then at $25^{\circ} \mathrm{C}$ for $15 \mathrm{~h}$. Then, $20 \mathrm{~mL}$ saturated aqueous $\mathrm{NH}_{4} \mathrm{Cl}$ was added into the reaction mixture. The organic phase was separated, and the aqueous phase was extracted with DCM $(3 \times 15 \mathrm{~mL})$. The combined organic phase was dried over $\mathrm{MgSO}_{4}$ and the solvent was removed under reduced pressure. The residue was purified by chromatography on silica gel eluting with hexanes/EtOAc (50/1-30/1 (v/v)) to afford $515 \mathrm{mg}$ of the acylated product $\mathbf{4 2}$ as a colorless liquid (81\% yield).

$\boldsymbol{R}_{\boldsymbol{f}}=0.60($ hexanes $/$ EtOAc $=10 / 1(\mathrm{v} / \mathrm{v}))$.

\section{NMR Spectroscopy:}

${ }^{1} \mathrm{H}$ NMR $\left(500 \mathrm{MHz}, \mathrm{CDCl}_{3}, 25^{\circ} \mathrm{C}, \delta\right): 5.80-5.69(\mathrm{~m}, 1 \mathrm{H}), 5.01-4.72(\mathrm{~m}, 3 \mathrm{H}), 3.94-3.80(\mathrm{~m}, 2 \mathrm{H}), 2.64$ $-2.47(\mathrm{~m}, 1 \mathrm{H}), 2.04(\mathrm{~d}, J=5.8 \mathrm{~Hz}, 1 \mathrm{H}), 2.00(\mathrm{~s}, 3 \mathrm{H}), 1.93$ (d, J = 7.7 Hz, 1H), $1.69(\mathrm{~s}, 3 \mathrm{H}), 1.60(\mathrm{~s}, 3 \mathrm{H})$, $1.55-1.45(\mathrm{~m}, 1 \mathrm{H}), 1.27-1.18(\mathrm{~m}, 1 \mathrm{H})$. 
${ }^{13} \mathrm{C}$ NMR $\left(126 \mathrm{MHz}, \mathrm{CDCl}_{3}, 25^{\circ} \mathrm{C}, \delta\right): 171.2,138.7,134.4,125.1,114.6,67.9,37.1,31.4,31.2,25.9$, 21.0, 18.3.

Both NMR data are consistent with the reported data. ${ }^{4}$

(3-((Cyclohexylamino)methyl)-2-(prop-1-en-2-yl)cyclopentyl)methyl acetate 43

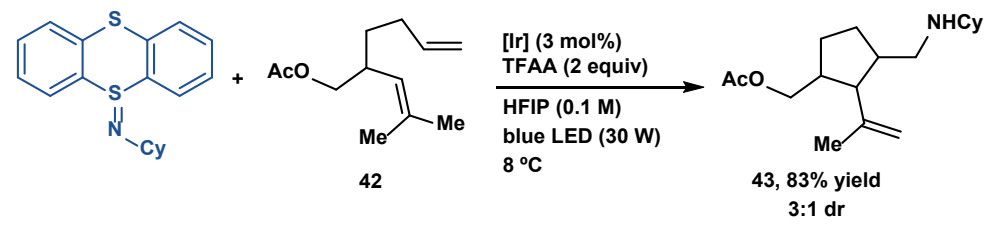

Under nitrogen atmosphere, to a 4-mL borosilicate vial equipped with a magnetic stir bar were added thianthrenylidene propylamine 3 (27.3 mg, $0.100 \mathrm{mmol}, 1.00$ equiv.), 42 (29.4 mg, $0.150 \mathrm{mmol}, 1.50$ equiv.), $\operatorname{Ir}\left[\mathrm{dF}\left(\mathrm{CF}_{3}\right) \mathrm{ppy}_{2}(\mathrm{dtbpy}) \mathrm{PF}_{6}(3.3 \mathrm{mg}, 3.0 \mu \mathrm{mol}, 3.0 \mathrm{~mol} \%)\right.$, HFIP $(1 \mathrm{~mL}, \mathrm{c}=0.1 \mathrm{M})$, and trifluoroacetic anhydride $\left(28 \mu \mathrm{L}, 42 \mathrm{mg}, 0.20 \mathrm{mmol}, 2.0\right.$ equiv.). The vial was sealed with a septum-cap and irradiated for $6 \mathrm{~h}$ at $8{ }^{\circ} \mathrm{C}$ using a blue LED $(30 \mathrm{~W})$. Then, the reaction mixture was concentrated. The residue was dissolved in DCM ( 5 $\mathrm{mL})$ and washed with saturated sodium carbonate aqueous solution $(5 \mathrm{~mL})$. The aqueous phase was extracted with DCM $(2 \times 5 \mathrm{~mL})$. The organic phase was dried over $\mathrm{Na}_{2} \mathrm{SO}_{4}$ and the solvent was removed under reduced pressure. The residue was purified by chromatography on silica gel eluting with $\mathrm{CHCl}_{3} / \mathrm{MeOH}$ $(50 / 1-30 / 1(v / v))$ to afford $24.5 \mathrm{mg}$ of $\mathbf{4 3}$ as a colorless oil (83\% yield, 3:1 dr).

$\mathbf{R}_{\boldsymbol{f}}=0.20\left(\mathrm{CHCl}_{3} / \mathrm{MeOH}=15 / 1(\mathrm{v} / \mathrm{v})\right)$.

\section{NMR Spectroscopy:}

${ }^{1} \mathrm{H}$ NMR (500 MHz, $\left.\mathrm{CDCl}_{3}, 25^{\circ} \mathrm{C}, \delta\right): 4.93$ (s, 1H), 4.77 (s, 1H), 4.10 (dd, J = 10.8, $4.3 \mathrm{~Hz}, 1 \mathrm{H}$ ), 3.83 (dd, $J=10.9,6.7 \mathrm{~Hz}, 1 \mathrm{H}), 2.83(\mathrm{t}, J=4.0 \mathrm{~Hz}, 1 \mathrm{H}), 2.76-2.67(\mathrm{~m}, 1 \mathrm{H}), 2.62-2.52(\mathrm{~m}, 1 \mathrm{H}), 2.51(\mathrm{~d}, J=4.1$ $\mathrm{Hz}, 1 \mathrm{H}), 2.36-2.29(\mathrm{~m}, 1 \mathrm{H}), 2.09-1.96(\mathrm{~m}, 5 \mathrm{H}), 1.87-1.75(\mathrm{~m}, 2 \mathrm{H}), 1.73(\mathrm{~s}, 3 \mathrm{H}), 1.46-1.35(\mathrm{~m}, 4 \mathrm{H})$, $1.27-1.08(\mathrm{~m}, 6 \mathrm{H})$.

${ }^{13} \mathrm{C}$ NMR $\left(151 \mathrm{MHz}, \mathrm{CDCl}_{3}, 25^{\circ} \mathrm{C}, \delta\right): 171.3,143.2,112.6,67.5,58.2,51.9,46.1,39.6,38.9,28.9,27.7$, 25.1, 24.8, 24.7, 23.6, 21.0.

HRMS-ESI(m/z) calc'd for $\mathrm{C}_{18} \mathrm{H}_{32} \mathrm{NO}_{2}[\mathrm{M}+1]^{+}, 294.2428$; found, 294.2428; deviation: -0.02 ppm.

N-Cyclohexyl-5H-dibenzo[b,d]thiophen-5-imine 44

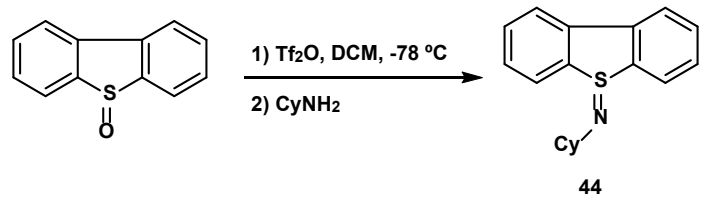

Under argon atmosphere, to a $50 \mathrm{~mL}$ two-necked round-bottomed flask were added dibenzothiophene oxide (400 mg, $2.00 \mathrm{mmol}, 1.00$ equiv.) and $20 \mathrm{~mL}$ dry DCM. The mixture was stirred at $-78{ }^{\circ} \mathrm{C}$ followed by addition of triflic anhydride ( $353 \mu \mathrm{L}, 592 \mathrm{mg}, 2.10 \mathrm{mmol}, 1.05$ equiv.). After stirring for $30 \mathrm{~min}$, to the reaction mixture 
was added cyclohexylamine $(570 \mu \mathrm{L}, 495 \mathrm{mg}, 5.00 \mathrm{mmol}, 2.50$ equiv. $)$ slowly. Then, the reaction was warmed to room temperature. Saturated sodium carbonate aqueous solution $(20 \mathrm{~mL})$ was added to the reaction mixture, which was then stirred for additional $5 \mathrm{~min}$. The resulting mixture was transferred to a separation funnel and extracted with DCM $(3 \times 20 \mathrm{~mL})$. The combined organic layers were dried over $\mathrm{Na}_{2} \mathrm{SO}_{4}$, and concentrated under reduced pressure. The obtained residue was purified by column chromatography on silica gel eluting with $\mathrm{DCM} / \mathrm{MeOH}(50 / 1-20 / 1(\mathrm{v} / \mathrm{v}))$ to afford $312 \mathrm{mg}$ of $\mathbf{4 4}$ as a colorless solid (55\% yield).

$\mathbf{R}_{\boldsymbol{f}}=0.15(\mathrm{DCM} / \mathrm{MeOH}=20 / 1(\mathrm{v} / \mathrm{v}))$.

m.m. $=127.6-128.4{ }^{\circ} \mathrm{C}$.

NMR Spectroscopy:

${ }^{1} \mathrm{H}$ NMR $\left(500 \mathrm{MHz}, \mathrm{CDCl}_{3}, 25^{\circ} \mathrm{C}, \delta\right): 7.96-7.90(\mathrm{~m}, 2 \mathrm{H}), 7.86(\mathrm{~d}, J=7.7 \mathrm{~Hz}, 2 \mathrm{H}), 7.58(\mathrm{td}, J=7.5,1.1$ $\mathrm{Hz}, 2 \mathrm{H}), 7.48$ (td, $J=7.5,1.1 \mathrm{~Hz}, 2 \mathrm{H}), 2.13$ (ddd, $J=10.3,6.2,4.1 \mathrm{~Hz}, 1 \mathrm{H}), 1.55-1.46(\mathrm{~m}, 2 \mathrm{H}), 1.44-$ $1.36(\mathrm{~m}, 2 \mathrm{H}), 1.35-1.30(\mathrm{~m}, 1 \mathrm{H}), 1.22-1.10(\mathrm{~m}, 2 \mathrm{H}), 1.07-0.94(\mathrm{~m}, 1 \mathrm{H}), 0.91-0.79(\mathrm{~m}, 2 \mathrm{H})$.

${ }^{13} \mathrm{C}$ NMR $\left(126 \mathrm{MHz} \mathrm{CDCl}_{3}, 25^{\circ} \mathrm{C}, \delta\right): 142.8,137.4,131.4,129.0,127.0,121.8,59.5,37.0,25.7,25.4$.

IR (neat, thin film): $V_{\max }\left(\mathrm{cm}^{-1}\right)=3039,2924,2844,1577,1440,1343,1259,1101,1083,1026,875,749$, 713.

HRMS-ESI(m/z) calc'd for $\mathrm{C}_{18} \mathrm{H}_{20} \mathrm{NS}[\mathrm{M}+\mathrm{H}]^{+}$, 282.1313; found, 282.1311; deviation: -0.76 ppm.

1,1-Bis(4-chlorophenyl)-N-cyclohexylsulfanimine 45

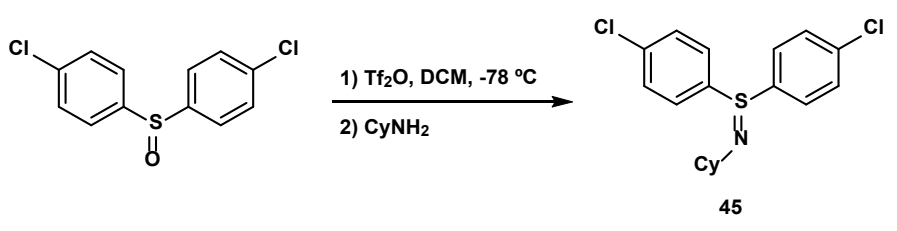

Under argon atmosphere, to a $50 \mathrm{~mL}$ two-necked round-bottomed flask were added 4,4'sulfinylbis(chlorobenzene) (540 mg, $2.00 \mathrm{mmol}, 1.00$ equiv.) and $20 \mathrm{~mL}$ dry DCM. The mixture was stirred at $-78{ }^{\circ} \mathrm{C}$ followed by addition of triflic anhydride ( $353 \mu \mathrm{L}, 592 \mathrm{mg}, 2.10 \mathrm{mmol}, 1.05$ equiv.). After stirring for 30 $\mathrm{min}$, to the reaction mixture was added cyclohexylamine $(570 \mu \mathrm{L}, 495 \mathrm{mg}, 5.00 \mathrm{mmol}, 2.50$ equiv.) slowly. Then, the reaction was warmed to room temperature. Saturated sodium carbonate aqueous solution $(20 \mathrm{~mL})$ was added to the reaction mixture, which was then stirred for additional $5 \mathrm{~min}$. The resulting mixture was transferred to a separation funnel and extracted with DCM $(3 \times 20 \mathrm{~mL})$. The combined organic layers were dried over $\mathrm{Na}_{2} \mathrm{SO}_{4}$, and concentrated under reduced pressure. The obtained residue was purified by column chromatography on silica gel eluting with $\mathrm{DCM} / \mathrm{MeOH}(50 / 1-20 / 1(\mathrm{v} / \mathrm{v}))$ to afford $475 \mathrm{mg}$ of $\mathbf{4 5}$ as a colorless solid $(67 \%$ yield).

$\boldsymbol{R}_{\boldsymbol{f}}=0.15(\mathrm{DCM} / \mathrm{MeOH}=20 / 1(\mathrm{v} / \mathrm{v}))$.

m.m. $=45.3-47.6{ }^{\circ} \mathrm{C}$. 


\section{NMR Spectroscopy:}

${ }^{1} \mathrm{H}$ NMR $\left(500 \mathrm{MHz}, \mathrm{CDCl}_{3}, 25{ }^{\circ} \mathrm{C}, \delta\right): 7.81(\mathrm{~d}, J=8.8 \mathrm{~Hz}, 4 \mathrm{H}), 7.60(\mathrm{~d}, J=8.8 \mathrm{~Hz}, 4 \mathrm{H}), 3.35-3.25(\mathrm{~m}$, $1 \mathrm{H}), 2.00-1.92(\mathrm{~m}, 2 \mathrm{H}), 1.81-1.73(\mathrm{~m}, 2 \mathrm{H}), 1.64-1.54(\mathrm{~m}, 1 \mathrm{H}), 1.54-1.42(\mathrm{~m}, 2 \mathrm{H}), 1.34-1.21(\mathrm{~m}$, $2 \mathrm{H}), 1.20-1.10(\mathrm{~m}, 1 \mathrm{H})$.

${ }^{13} \mathrm{C}$ NMR $\left(126 \mathrm{MHz}, \mathrm{CDCl}_{3}, 25^{\circ} \mathrm{C}, \delta\right): 141.5,131.6,130.2,129.8,59.2,33.8,24.9,24.9$.

IR (neat, thin film): $V_{\max }\left(\mathrm{cm}^{-1}\right)=3099,2930,2852,1570,1475,1399,1278,1244,1227,1151,1094,1029$, $1009,819,747,638$.

HRMS-ESI(m/z) calc'd for $\mathrm{C}_{18} \mathrm{H}_{20} \mathrm{NSCl}_{2}[\mathrm{M}+\mathrm{H}]^{+}$, 352.0688; found, 352.0688; deviation: $-0.02 \mathrm{ppm}$.

Thianthrene TT
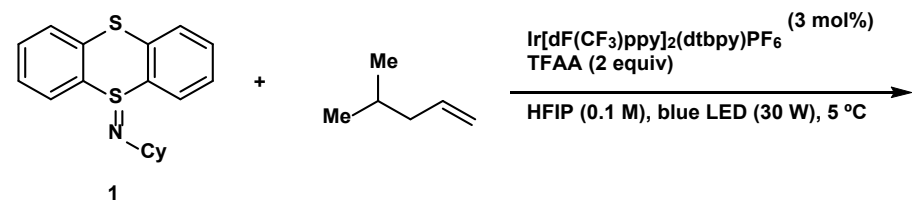

1

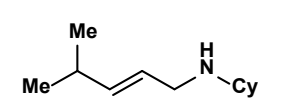

11

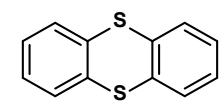

TT

Under nitrogen atmosphere, to a 4-mL borosilicate vial equipped with a magnetic stir bar were added thianthrenylidene cyclohexylamine 1 (31.3 mg, $0.100 \mathrm{mmol}, 1.00$ equiv.), 4-methyl-1-pentene (19.0 $\mu \mathrm{L}, 12.6$ $\mathrm{mg}, 0.150 \mathrm{mmol}, 1.50$ equiv.), $\operatorname{Ir}\left[\mathrm{dF}\left(\mathrm{CF}_{3}\right) \mathrm{ppy}\right]_{2}(\mathrm{dtbpy}) \mathrm{PF} 6$ (3.3 mg, $\left.3.0 \mu \mathrm{mol}, 3.0 \mathrm{~mol} \%\right), \mathrm{HFIP}(1 \mathrm{~mL}, \mathrm{c}=0.1$ $\mathrm{M})$, and trifluoroacetic anhydride ( $28 \mu \mathrm{L}, 42 \mathrm{mg}, 0.20 \mathrm{mmol}, 2.0$ equiv.). The vial was sealed with a septumcap and irradiated for $3 \mathrm{~h}$ at $8{ }^{\circ} \mathrm{C}$ using a blue LED (30 W). Then, the reaction mixture was concentrated. The residue was purified by chromatography on silica gel eluting with hexanes/EtOAc (50/1-30/1 (v/v)) to afford $18.6 \mathrm{mg}$ of TT as a colorless solid ( $86 \%$ yield).

$\mathbf{R}_{\boldsymbol{f}}=0.25($ hexanes $/$ EtOAc $=20 / 1(\mathrm{v} / \mathrm{v}))$.

\section{NMR Spectroscopy:}

${ }^{1} \mathrm{H}$ NMR $\left(500 \mathrm{MHz}, \mathrm{CDCl}_{3}, 25^{\circ} \mathrm{C}, \delta\right): 7.49$ (dd, $\left.J=5.7,3.4 \mathrm{~Hz}, 4 \mathrm{H}\right), 7.24(\mathrm{dd}, J=5.8,3.4 \mathrm{~Hz}, 4 \mathrm{H})$.

The NMR data is consistent with the reported data. ${ }^{5}$ 
SUPPORTING INFORMATION

S54

Mechanistic investigations for allylic $\mathrm{C}-\mathrm{H}$ amination

Wavelength of the LED lights

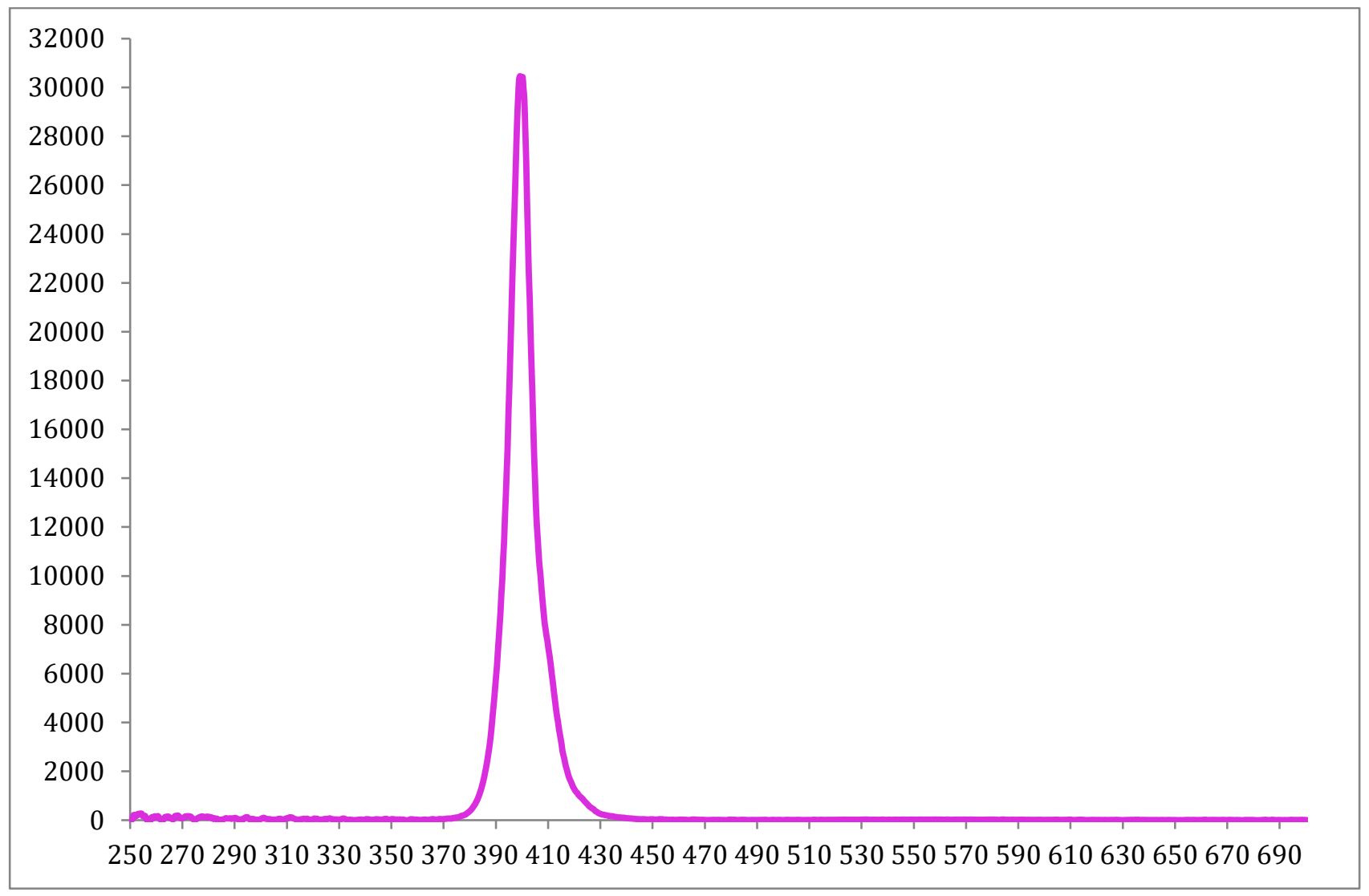

Figure S4. Emission spectrum of the purple LED lamp. 


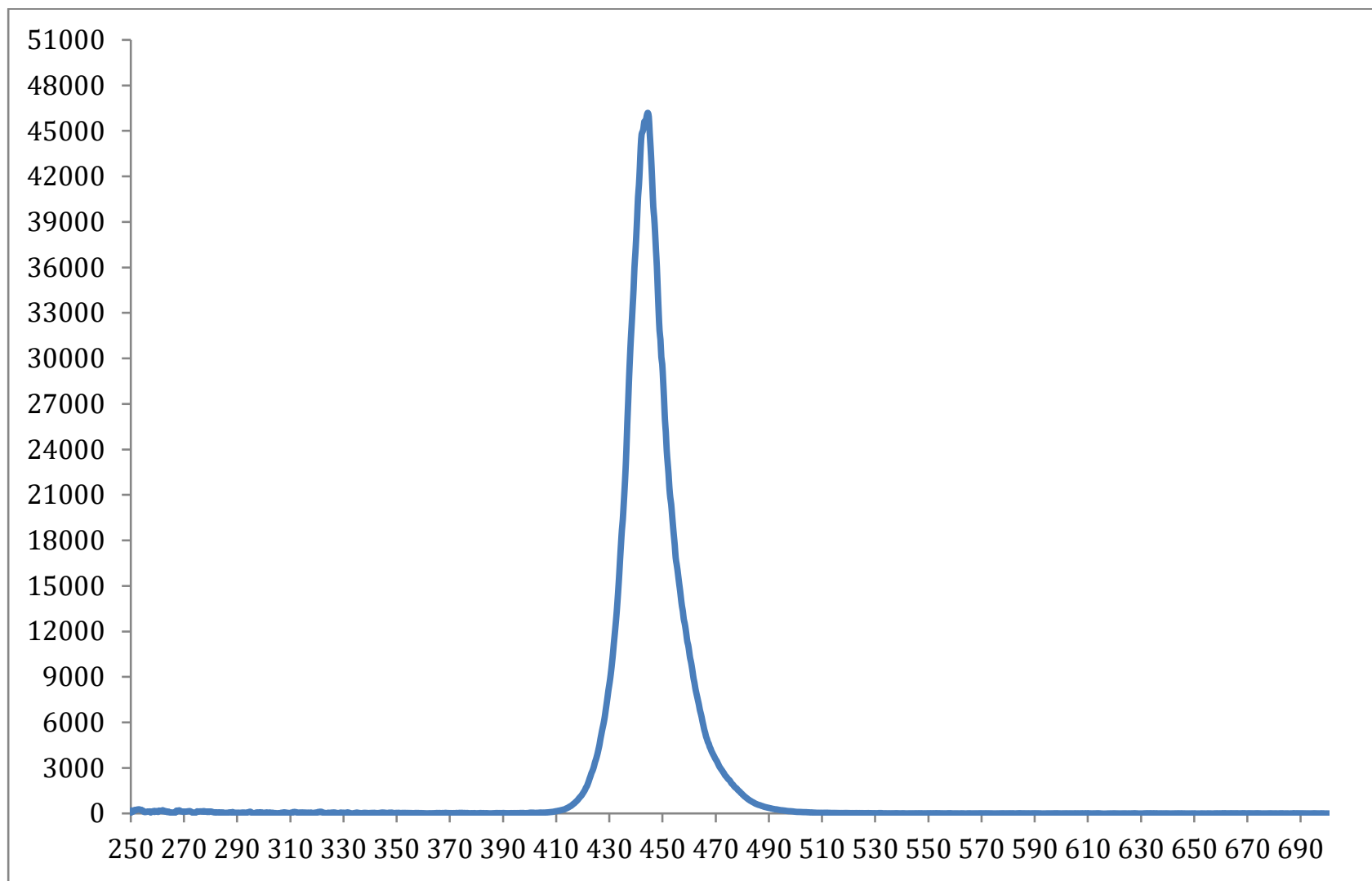

Figure S5. Emission spectrum of the blue LED lamp.

\section{Stern-Volmer Luminescence Quenching Studies}

Visible light luminescence intensities were recorded using an Edinburgh Instruments FS5 spectrofluorometer. All luminescence measurements were recorded using a screw-top quartz cuvette (Hellma fluorescence quartz cuvette, $10 \times 10 \mathrm{~mm}, 3.5 \mathrm{~mL})$. All solutions of $\operatorname{Ir}\left[\mathrm{dF}\left(\mathrm{CF}_{3}\right) \mathrm{ppy}\right]_{2}(\mathrm{dtbpy}) \mathrm{PF}_{6}$, thianthrenylidene cyclohexylamine 1, and TFAA were prepared in HFIP in a nitrogen-filled glovebox. The solutions were transferred to the screw-top cuvette inside the glovebox, the cuvette was sealed, and then brought out of the glovebox for visible light luminescence measurements.

In a typical procedure, $1(0.125 \mathrm{~g}, 0.400 \mathrm{mmol})$ was dissolved and diluted to a final volume of $10 \mathrm{~mL}(\mathrm{c}=0.040$

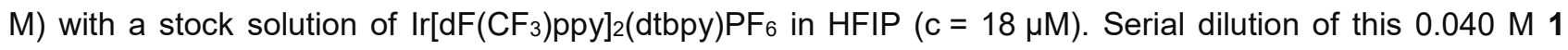
solution was carried out by dilution of $7 \mathrm{~mL}$ of the $0.040 \mathrm{M} 1$ solution to $10 \mathrm{~mL}(0.028 \mathrm{M})$ with the $18 \mu \mathrm{M}$ stock solution of $\operatorname{Ir}\left[\mathrm{dF}\left(\mathrm{CF}_{3}\right) \mathrm{ppy}\right]_{2}(\mathrm{dtbpy}) \mathrm{PF}_{6}$. All subsequent solutions were prepared by dilution of $7 \mathrm{~mL}$ of the preceding solution to a final volume of $10 \mathrm{~mL}$. All solutions were excited at $400 \mathrm{~nm}$ and the emission was measured from 450 to $600 \mathrm{~nm}$.

Quenching was analyzed by plotting Io/l according to the Stern-Volmer relationship:

$\mathrm{I} / \mathrm{l}=k_{\mathrm{q}} \mathrm{T}[\mathrm{Q}]+1$

where lo represents the integral of the luminescence over the range of 450 to $600 \mathrm{~nm}$ in the absence of a 
quencher, I is the integral of luminescence over the range of 450 to $600 \mathrm{~nm}$ in the presence of a quencher, $k_{\mathrm{q}}$ represents the quenching rate constant, $[\mathrm{Q}]$ is the concentration of a given quencher, and To is the excited state lifetime of the emissive photocatalyst in the absence of quencher.
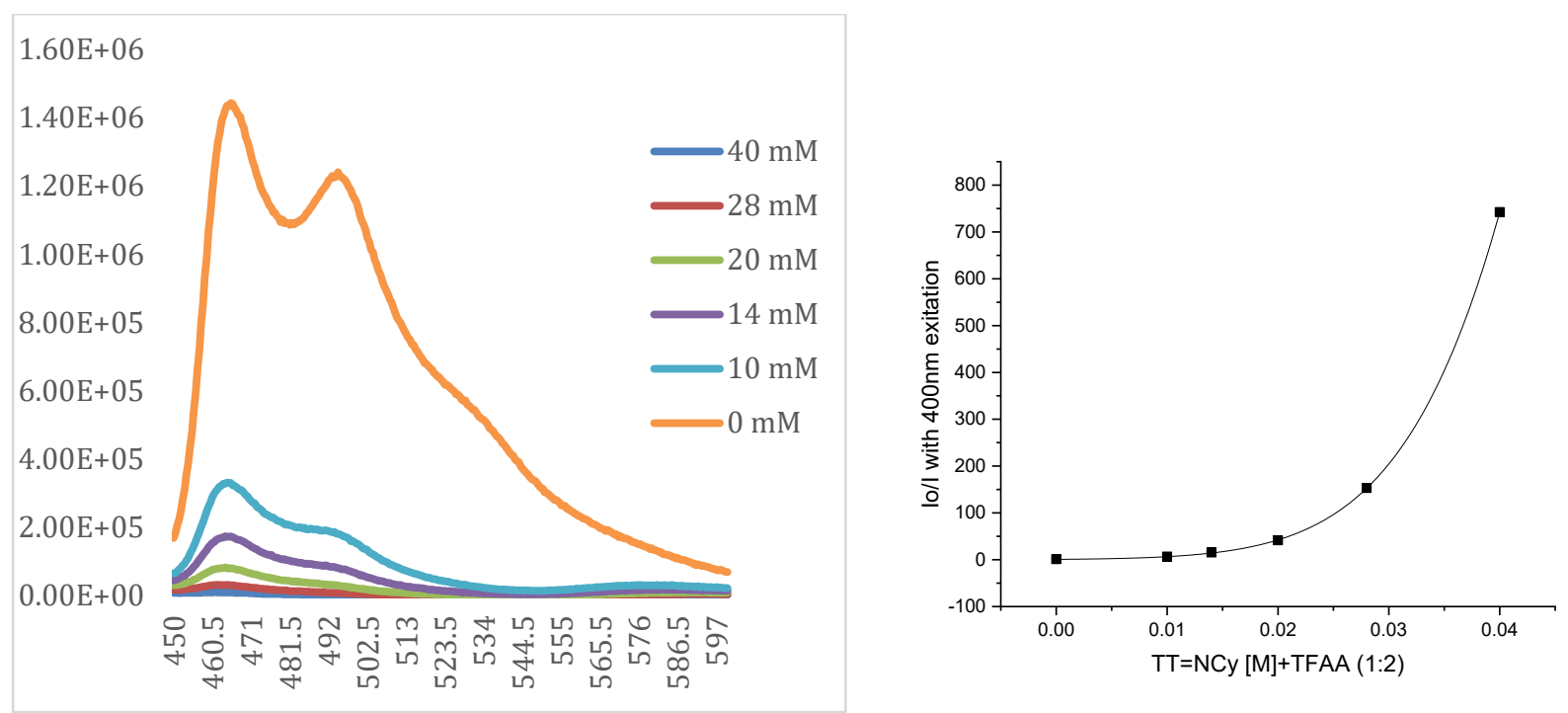

Figure S6. Emission spectra for $\operatorname{Ir}\left(\mathrm{dF}\left(\mathrm{CF}_{3}\right) \mathrm{ppy}\right)_{2}(\mathrm{dtbbpy}) \mathrm{PF}_{6}$ luminescence quenching by thianthrenylidene cyclohexylamine 1 with TFAA (2 equiv) as additive (left) and Stern-Volmer plot (right).
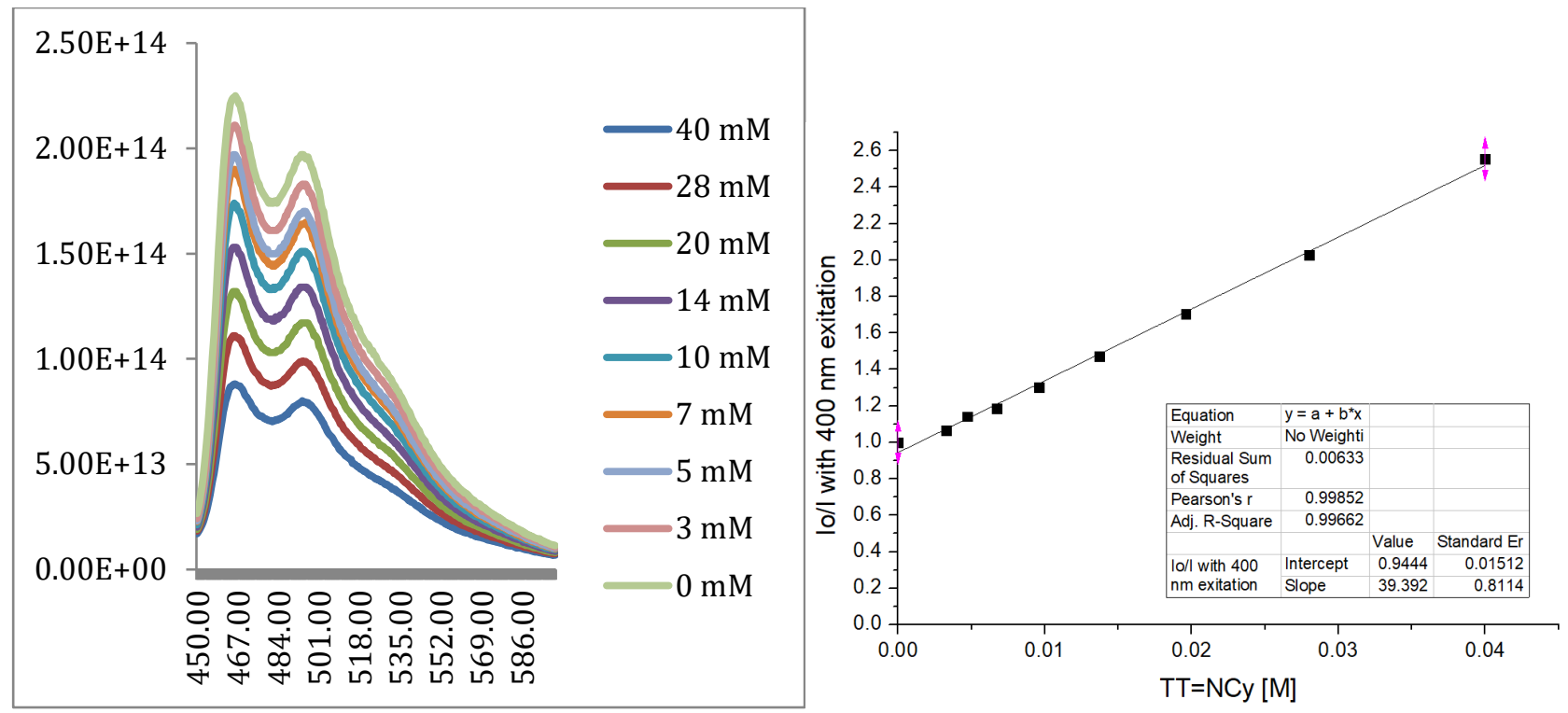

Figure S7. Emission spectra for $\operatorname{Ir}\left(\mathrm{dF}\left(\mathrm{CF}_{3}\right) \text { ppy }\right)_{2}(\mathrm{dtbbpy}) \mathrm{PF}_{6}$ luminescence quenching by thianthrenylidene cyclohexylamine 1 (left) and Stern-Volmer plot (right). 

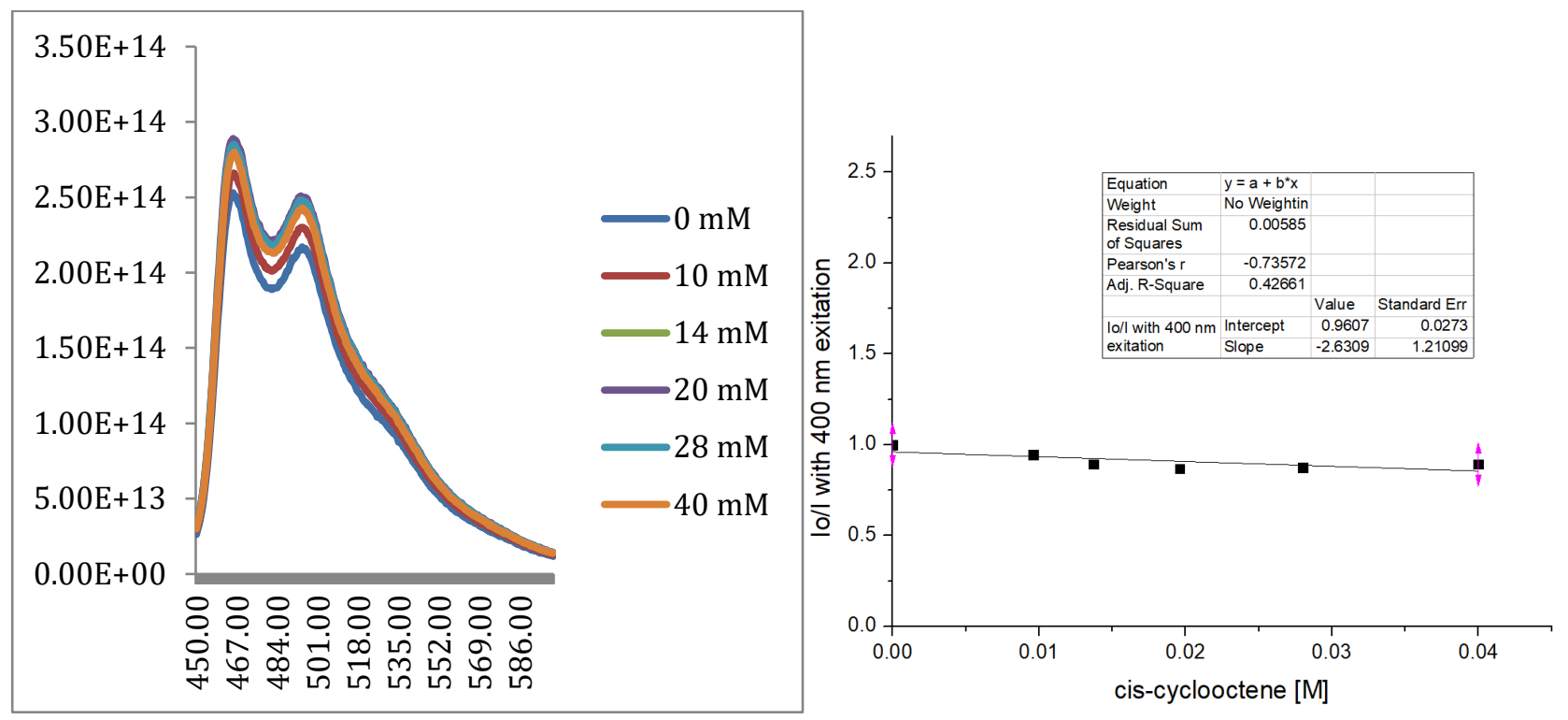

Figure S8. Emission spectra for $\operatorname{Ir}\left(\mathrm{dF}\left(\mathrm{CF}_{3}\right) \mathrm{ppy}\right)_{2}\left(\mathrm{dtbbpy} \mathrm{PF}_{6}\right.$ luminescence quenching by cis-cyclooctene (left) and Stern-Volmer plot (right).

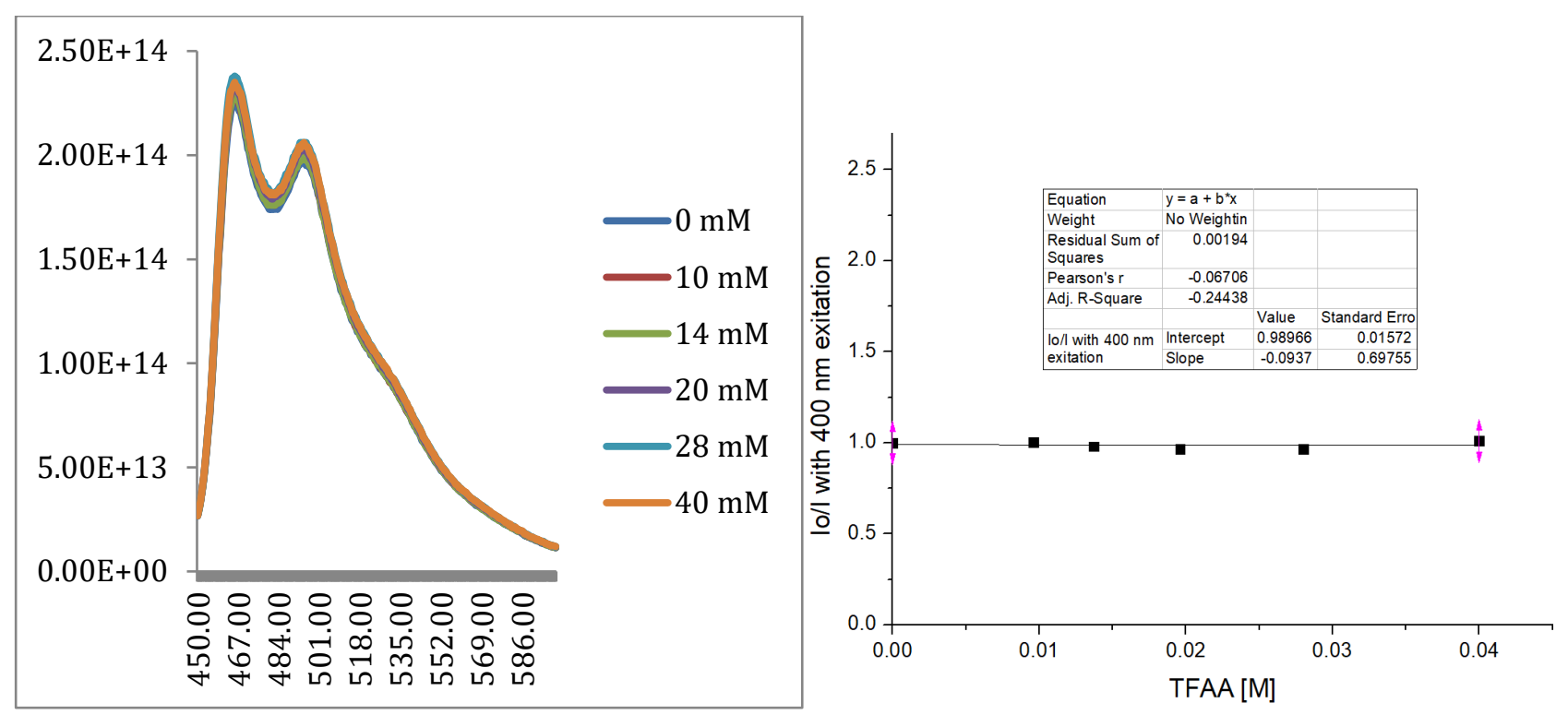

Figure S9. Emission spectra for $\operatorname{Ir}\left(\mathrm{dF}\left(\mathrm{CF}_{3}\right) \mathrm{ppy}\right)_{2}(\mathrm{dtbbpy}) \mathrm{PF}_{6}$ luminescence quenching by TFAA (left) and Stern-Volmer plot (right). 

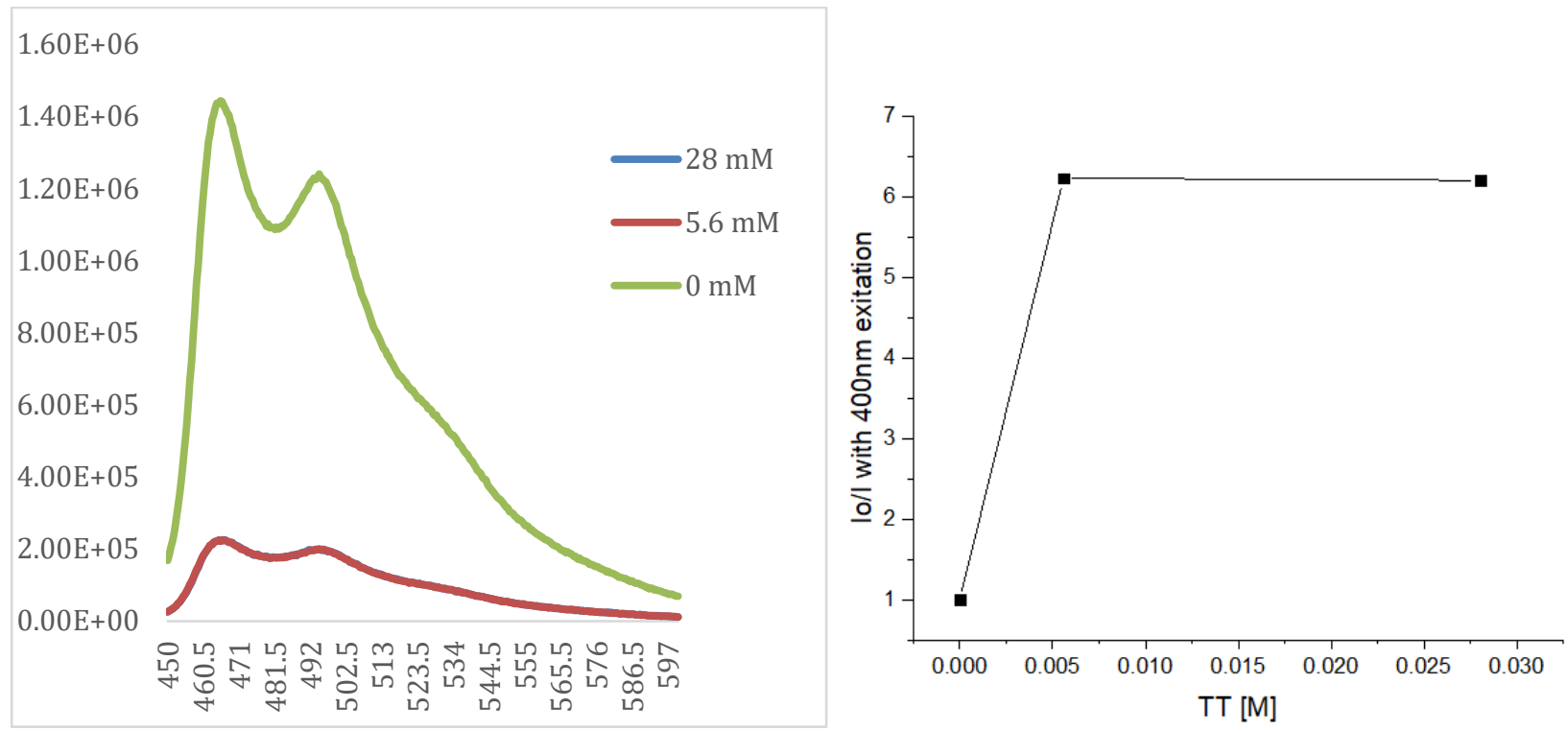

Figure S10. Emission spectra for $\operatorname{Ir}\left(\mathrm{dF}\left(\mathrm{CF}_{3}\right) \text { ppy }\right)_{2}(\mathrm{dtbbpy}) \mathrm{PF}_{6}$ luminescence quenching by $\mathrm{TT}$ (left) and SternVolmer plot (right). The solution is saturated at a concentration of TT in HFIP at $5.6 \mathrm{mM}$.

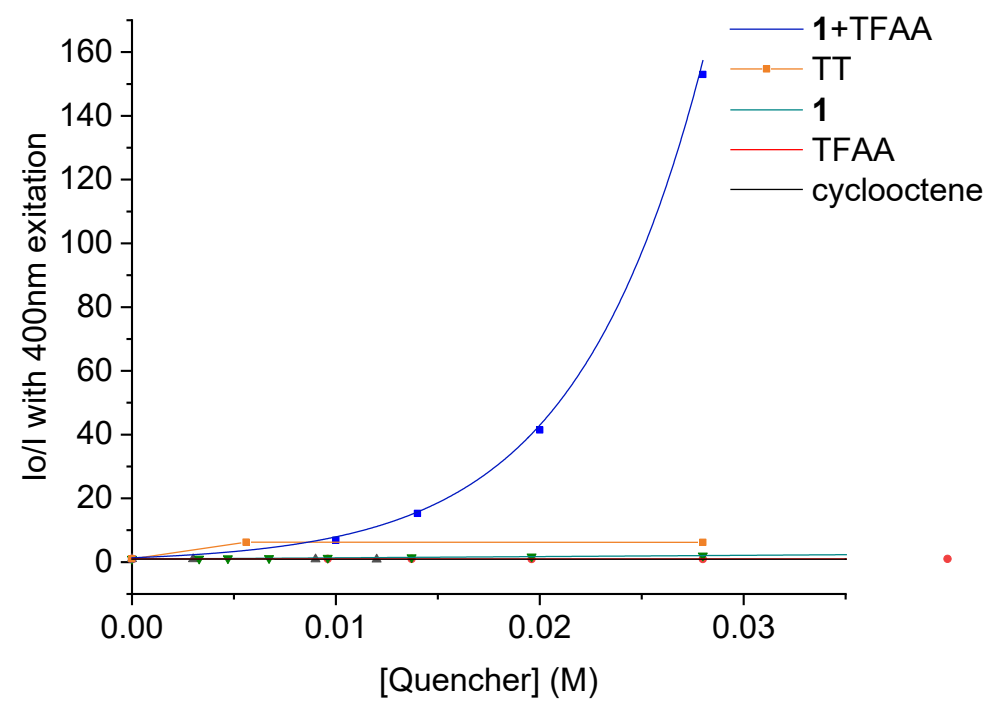

Figure S11. Stern-Volmer quenching experiments.

Cyclic voltammograms of thianthrenylidene cyclohexylamine 1

Cyclic voltammograms were recorded using an Autolab PGSTAT204 potentiostat and a Pt working electrode, a $\mathrm{Ag} / \mathrm{AgCl}$ reference electrode and a Pt sheet auxiliary electrode. The voltammograms were recorded at room temperature in $0.1 \mathrm{M}$ tetrabutylammonium hexafluorophosphate in HFIP $(3 \mathrm{~mL})$ containing thianthrenylidene cyclohexylamine 1 ( $9.4 \mathrm{mg}, 0.030 \mathrm{mmol})$ and TFAA (13 mg, $0.060 \mathrm{mmol})$. The scan rate was $100 \mathrm{mV} \mathrm{s}-1$. 

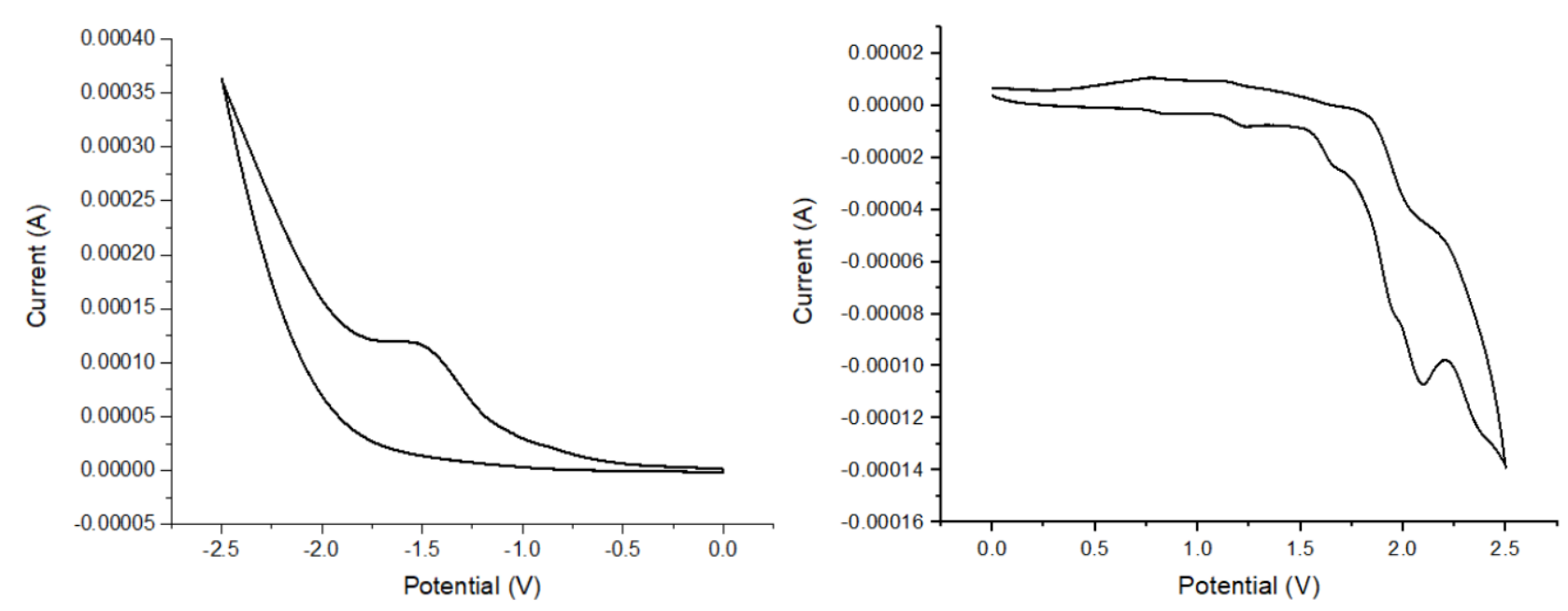

Figure S12. Cyclic Voltammetry for reduction (left) and oxidation (right) of 1 in HFIP with TFAA. An oxidation peak at $E_{o x}=2.0 \mathrm{~V}$ and reduction peak at $E_{\text {red }}=-1.4 \mathrm{~V}$ are observed.

\section{EPR Measurements}

\section{EPR experiment}

Electron paramagnetic resonance (EPR) spectra were obtained using a commercial X-band spectrometer (MS5000, Magnettech $\mathrm{GmbH}$ ) at $100 \mathrm{~K}$. The EPR spectra were recorded at microwave frequency of 9.473 $\mathrm{GHz}$ using $0.1 \mathrm{~mW}$ microwave power, $20 \mathrm{mT}$ field sweep centered at $336.8 \mathrm{mT}$, a modulation amplitude of $0.1 \mathrm{mT}$ and a sweep time $20 \mathrm{~s}$.

In a nitrogen-filled glovebox, 1 (15.6 mg, $50.0 \mu \mathrm{mol})$, allylbenzene $(10 \mu \mathrm{L}, 8.8 \mathrm{mg}, 75 \mu \mathrm{mol})$, $\operatorname{Ir}\left[\mathrm{dF}\left(\mathrm{CF}_{3}\right) \mathrm{ppy}\right]_{2}(\mathrm{dtbpy}) \mathrm{PF}_{6}(1.6 \mathrm{mg}, 1.5 \mu \mathrm{mol})$, trifluoroacetic anhydride $(14 \mu \mathrm{L}, 21 \mathrm{mg}, 0.10 \mathrm{mmol})$ was dissolved in HFIP $(0.5 \mathrm{~mL}, \mathrm{c}=0.1 \mathrm{M})$ before being transferred to a $4 \mathrm{~mm}$ (O.D.) EPR tube. The tube was sealed with a rubber septum and brought out of the glovebox. The tube was placed into a Dewar and held upright using a test tube rack. Two blue LEDs were angled into the Dewar, and a fan was placed above to cool the EPR tube $\left(28-30^{\circ} \mathrm{C}\right)$. After $10 \mathrm{~min}$ of irradiation, liquid nitrogen was poured into the Dewar, freezing the sample. The sample was kept in liquid nitrogen until it could be measured.

\section{Results and discussion}

EPR measurements for S2 and S3 samples displayed the formation of a radical signal with a rhombic $g$ tensor $(2.017,2.012$ and 2.006) (Figure S13). This radical signal matches very well with the previously observed thianthrene (TT) radical signal from the literature. ${ }^{6}$ Therefore, it is assigned to TT radical cation. 


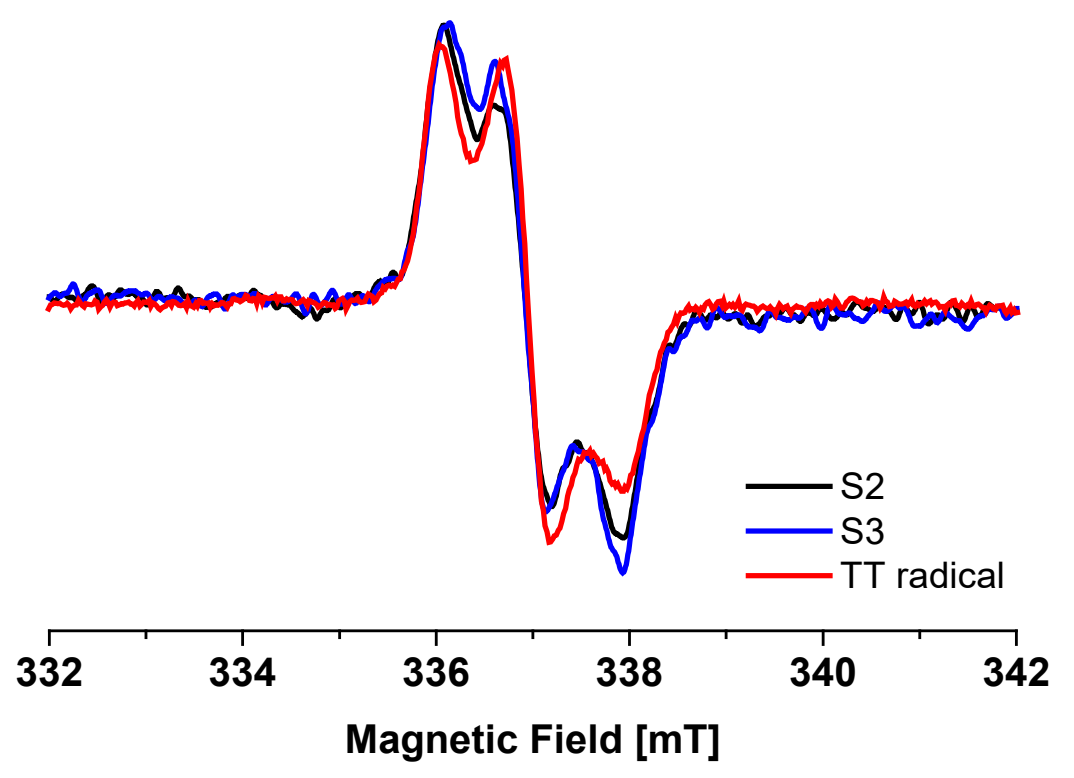

Figure S13. X-band EPR spectra recorded at $100 \mathrm{~K}$ are shown for S2 (a mixture of Ir, 1, allylbenzene, and TFAA after irradiation, black), S3 (a mixture of Ir, 1, and TFAA after irradiation, blue) and TT radical (red).

UV-Vis Spectrum of 1

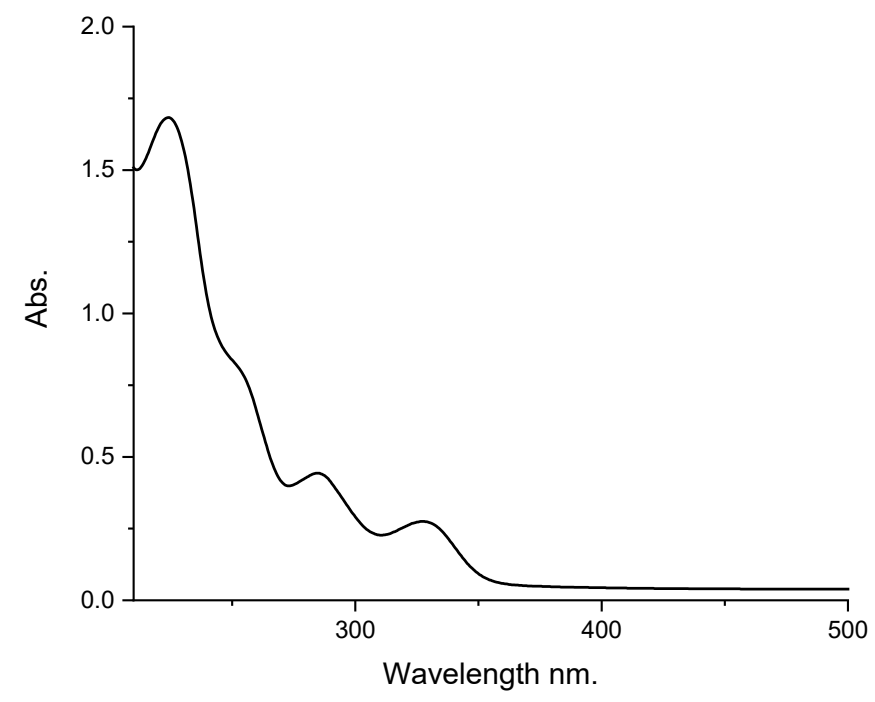

Figure S14. UV-Vis spectrum of 1 with TFAA in HFIP. 
Proposed mechanism for the formation of regioisomers when using cyclooctene as substrate

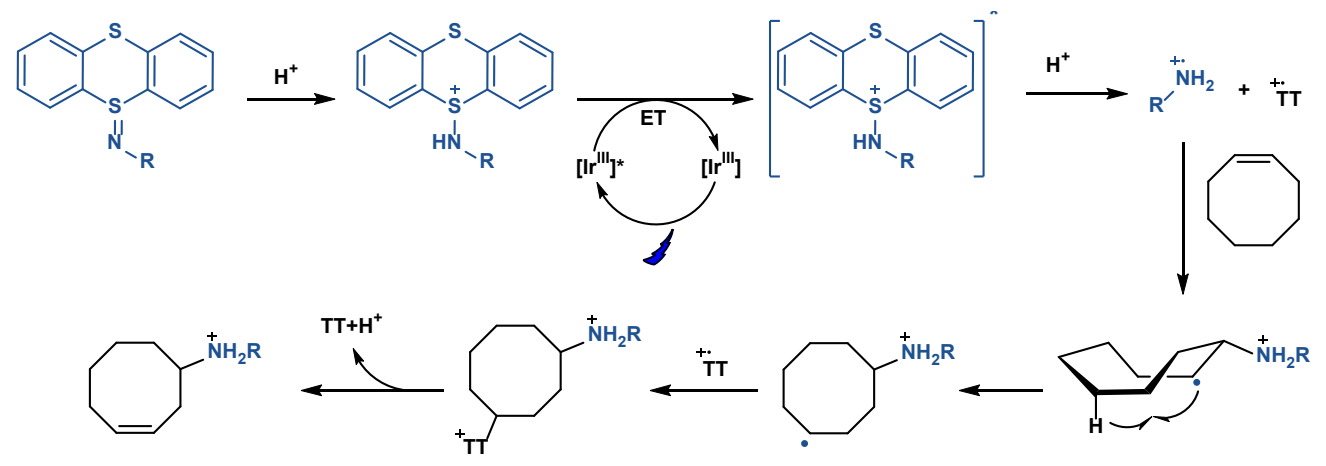

Figure S15. Proposed mechanism for homoallylic amine as minor product when using cyclooctene as substrate.

Procedure for isomerization of $(E)$-cinnamylamine 46 under photocatalysis

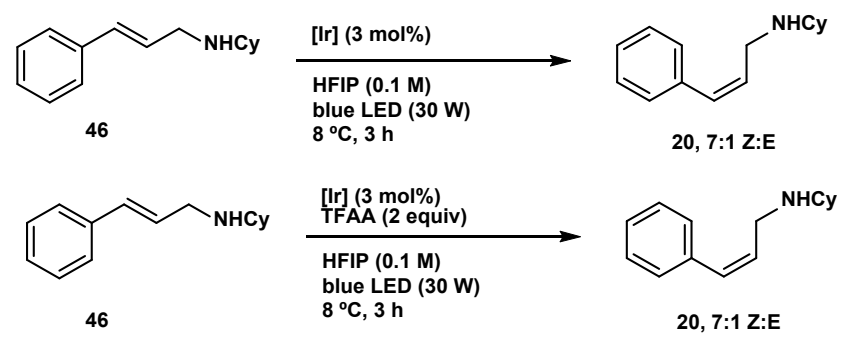

Under nitrogen atmosphere, to a 4-mL borosilicate vial equipped with a magnetic stir bar were added $(E)$ cinnamylamine 46 (21.5 mg, 0.100 mmol, 1.00 equiv.), $\operatorname{Ir}\left[\mathrm{dF}\left(\mathrm{CF}_{3}\right) \text { ppy }\right]_{2}(\mathrm{dtbpy}) \mathrm{PF} 6$ (3.3 mg, $3.0 \mu \mathrm{mol}, 3.0$ mol\%), HFIP ( $1 \mathrm{~mL}, \mathrm{c}=0.1 \mathrm{M}$ ), (and trifluoroacetic anhydride ( $28 \mu \mathrm{L}, 42 \mathrm{mg}, 0.20 \mathrm{mmol}, 2.0$ equiv.)). The vial was sealed with a septum-cap and irradiated for $3 \mathrm{~h}$ at $8{ }^{\circ} \mathrm{C}$ using a blue LED $(30 \mathrm{~W})$. Then, the reaction mixture was concentrated. The residue was directly dissolved in $\mathrm{CDCl}_{3}$ for ${ }^{1} \mathrm{H}$ NMR to determine the stereoselectivity. 


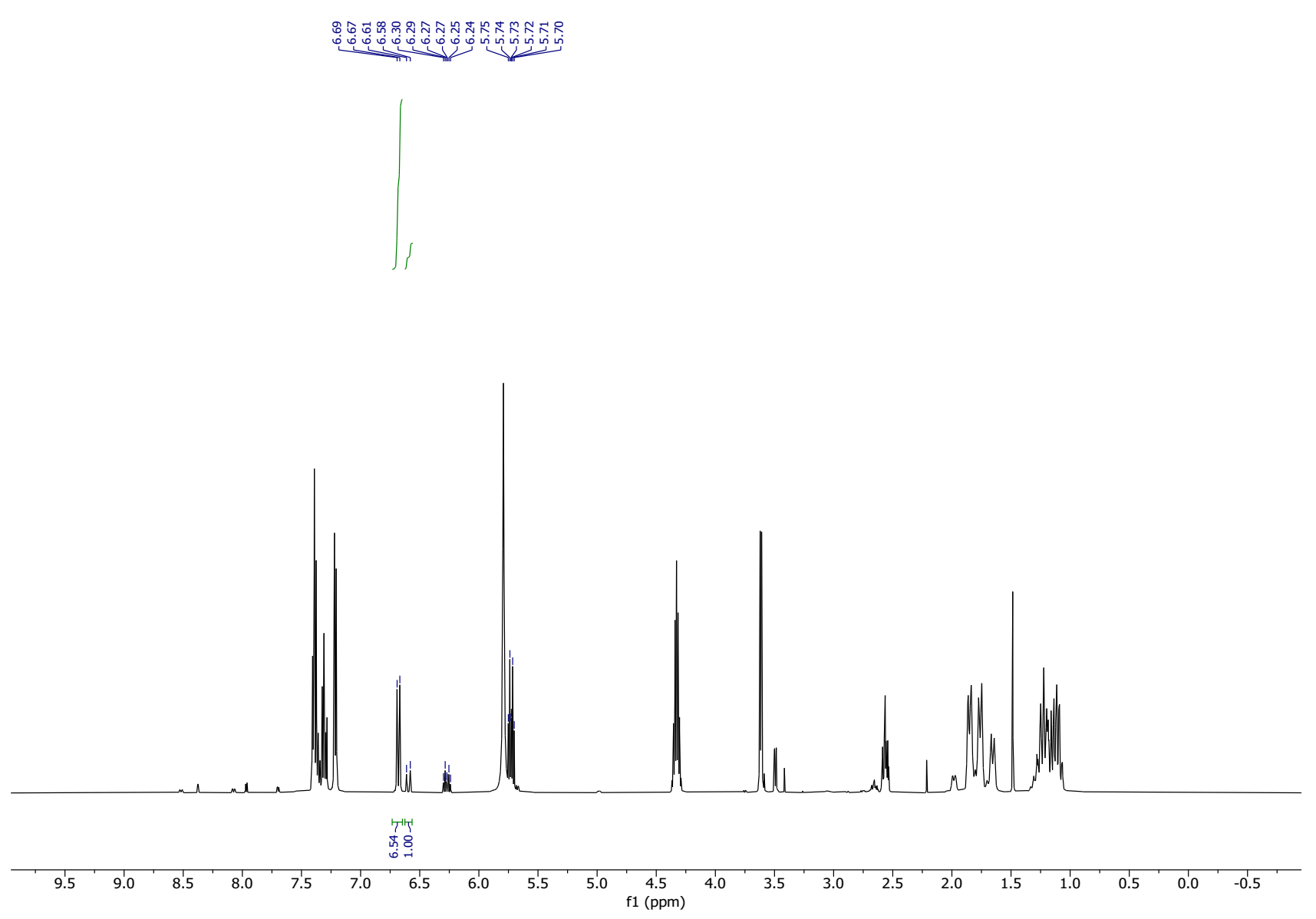

Figure S16. The crude ${ }^{1} \mathrm{H}$ NMR in $\mathrm{CDCl}_{3}$ of the reaction without TFAA. The ratio was determined as Z:E = 7:1. 


\section{$\underbrace{-10}$}

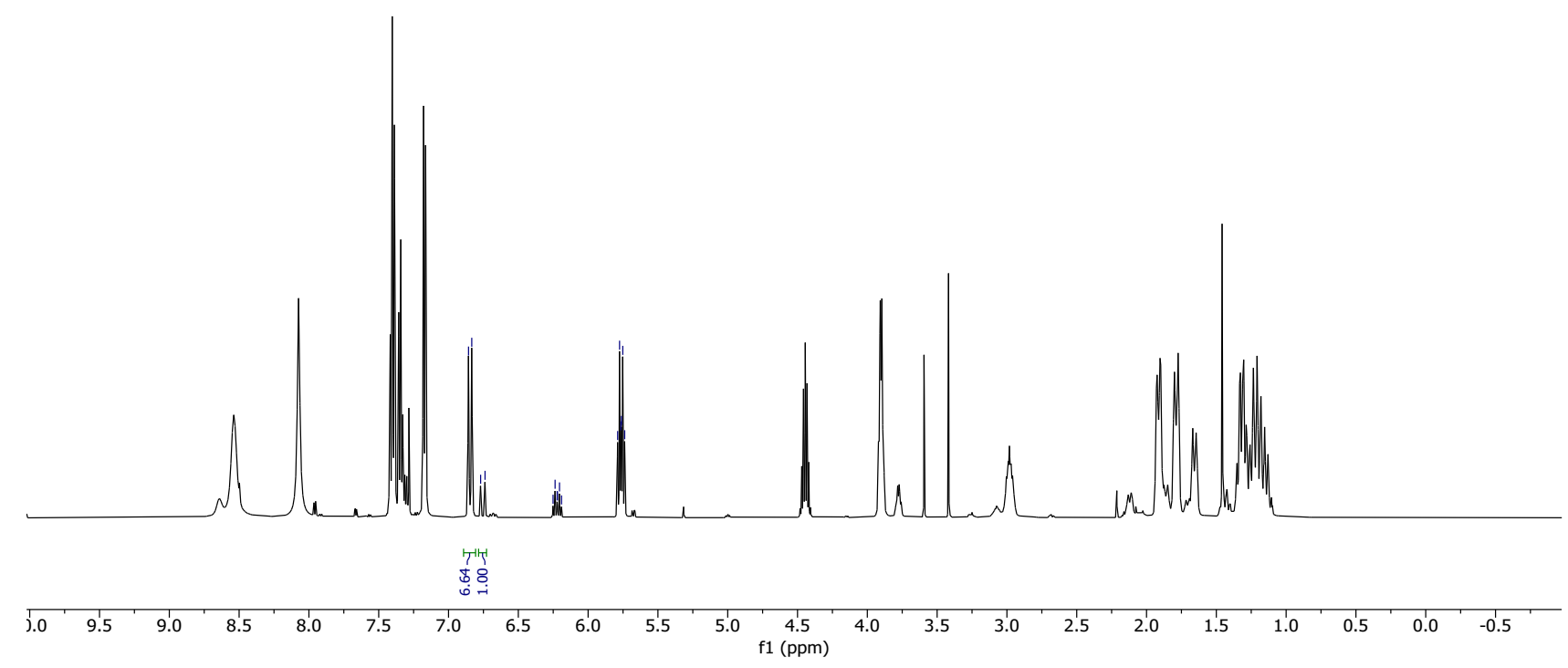

Figure S17. The crude ${ }^{1} \mathrm{H}$ NMR in $\mathrm{CDCl}_{3}$ of the reaction with TFAA. The ratio was determined as Z:E = 7:1.

Procedure for reaction under UV light

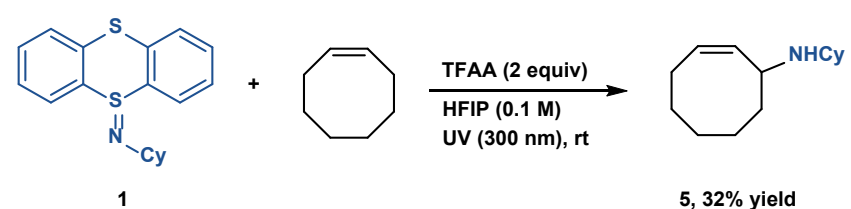

Under nitrogen atmosphere, to a $10 \mathrm{~mL}$ quartz vial equipped with a magnetic stir bar were added thianthrenylidene cyclohexylamine 1 (31.3 mg, $0.100 \mathrm{mmol}, 1.00$ equiv.), cis-cyclooctene (200 $\mu \mathrm{L}, 165 \mathrm{mg}$, $1.50 \mathrm{mmol}, 1.50$ equiv.), HFIP ( $1 \mathrm{~mL}, \mathrm{c}=0.1 \mathrm{M})$, and trifluoroacetic anhydride $(28 \mu \mathrm{L}, 42 \mathrm{mg}, 0.20 \mathrm{mmol}, 2.0$ equiv.). The vial was sealed with a septum-cap and irradiated for $3 \mathrm{~h}$ at room temperature using $8 \times 5 \mathrm{~W} U \mathrm{~V}$ light $(300 \mathrm{~nm})$. Then, the reaction mixture was concentrated. The residue was dissolved in DCM $(5 \mathrm{~mL})$ and washed with saturated sodium carbonate aqueous solution $(5 \mathrm{~mL})$. The aqueous phase was extracted with DCM $(2 \times$ $5 \mathrm{~mL}$ ). The organic phase was dried over $\mathrm{Na}_{2} \mathrm{SO}_{4}$ and the solvent was removed under reduced pressure. The residue was purified by chromatography on silica gel eluting with $\mathrm{CHCl}_{3} / \mathrm{MeOH}(30 / 1-10 / 1(\mathrm{v} / \mathrm{v}))$ to afford 6.6 $\mathrm{mg}$ of $\mathbf{5}$ as a colorless oil ( $32 \%$ yield).

\section{Discussion on other mechanistic possibilities}

1. The reaction might involve a trifluoroacetamide radical $\mathbf{E}$ from energy transfer between excited iridium 
photocatalyst and $\mathbf{D}$. The nitrogen-centered radical $\mathbf{E}$ is also electrophilic, and undergoes a similar reaction pathway to form allyl amide. Hydrolysis of the amide produces the same product (Figure S18). However, reaction without TFAA or with only TFA as additive also occurs (Table S1 and S5). In addition, no trifluoroacetamide was obtained when mixing the alkyl allylamine in HFIP with TFAA (Figure S19). The synthesized allyl trifluoroacetic amide $\mathbf{4 7}$ is also fully remained in both standard reaction conditions and basic work-up (Figure S20). All the evidences exclude the possibility that the trifluoroacetamide radical is the key intermediate.

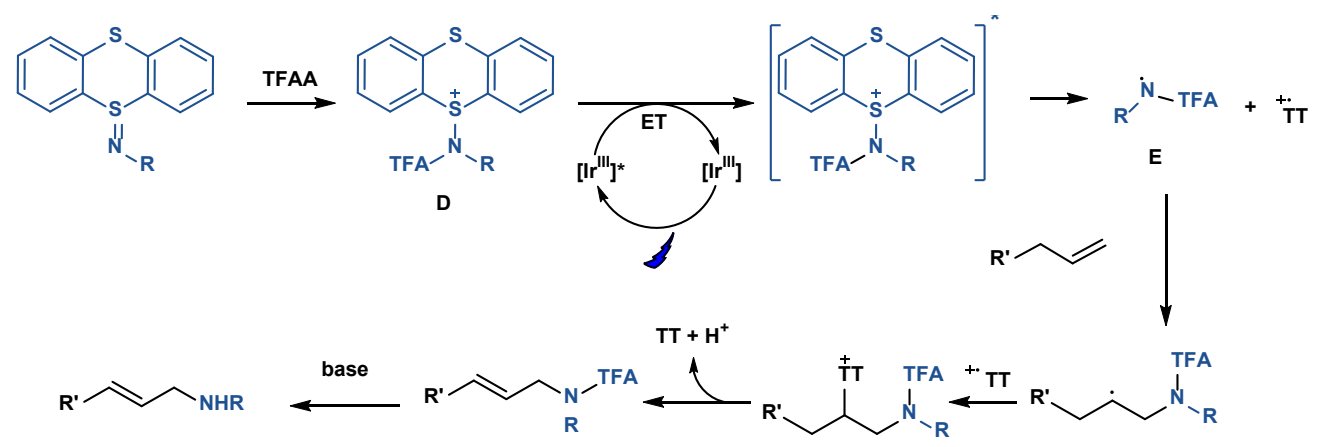

Figure S18. Proposed mechanism via a trifluoroacetamide radical.

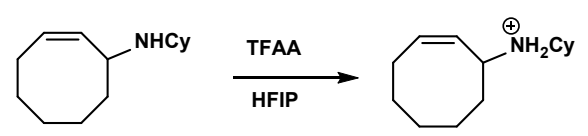

Figure S19. Reaction of allylamine with TFAA in HFIP.

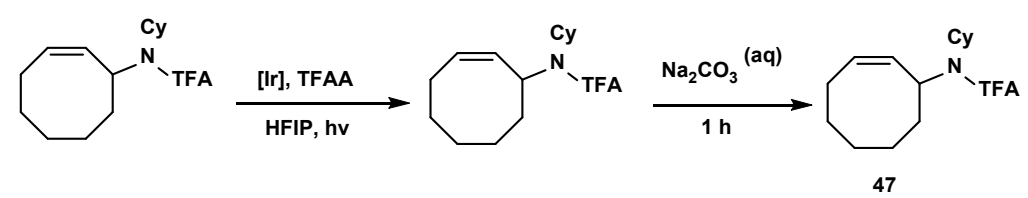



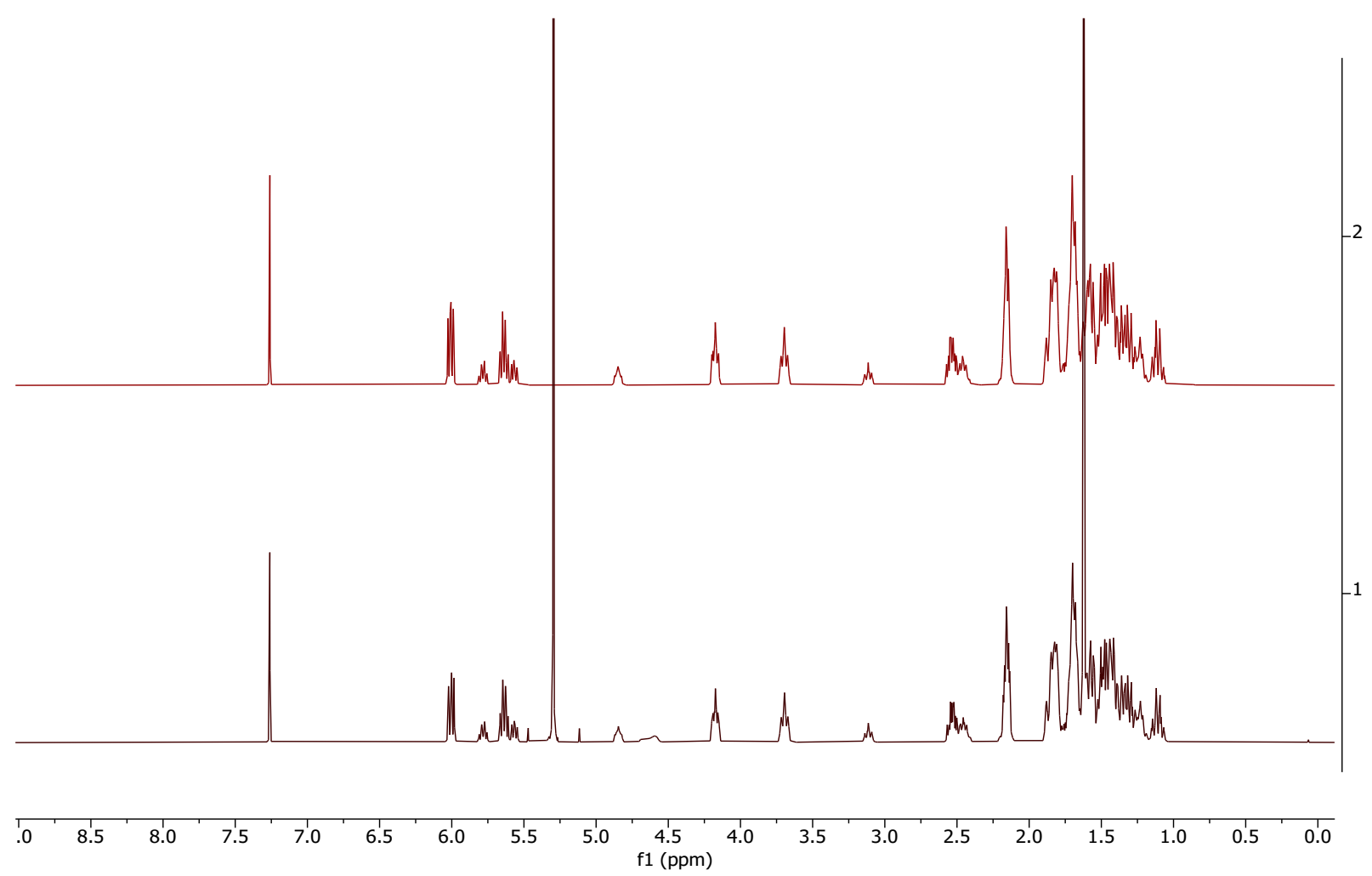

Figure S20. Stability of 47 in the reaction conditions and basic work-up. ${ }^{1} \mathrm{H}$ NMR spectra of the pure compound 47 with 3:1 conformational isomers (2) and the compound after reaction and work-up with saturated sodium carbonate aqueous solution (1).

Studies on the reaction between TFAA and HFIP<smiles>O=C(O)C=Cc1ccc(F)cc1</smiles>

(2.0 equiv) (1.0 equiv)

Under air, to a 4-mL borosilicate vial equipped with a magnetic stir bar were added HFIP $(210 \mu \mathrm{L}, 316 \mathrm{mg}$, $2.00 \mathrm{mmol}, 2.00$ equiv.) and trifluoroacetic anhydride $(210 \mathrm{mg}, 139 \mu \mathrm{L}, 1.00 \mathrm{mmol}, 1.00$ equiv.). The reaction mixture was stirred at $25^{\circ} \mathrm{C}$, and aliquots were taken from the reaction mixture, diluted with $4 \mathrm{~mL}$ of MeCN and analyzed by ${ }^{19} \mathrm{~F}$ NMR spectroscopy (Figure S21). 


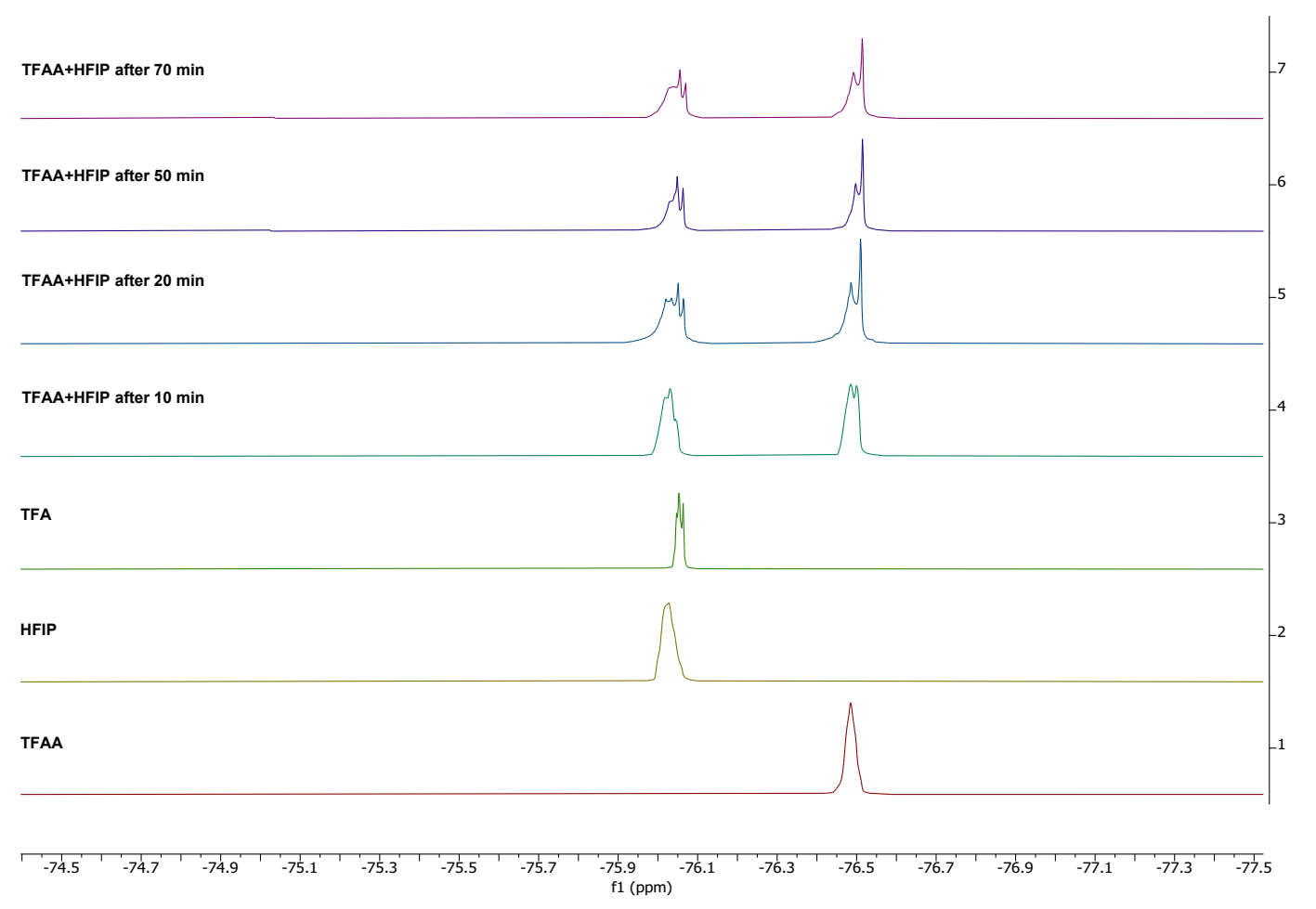

Figure S21. ${ }^{19} \mathrm{~F}$ NMR of TFAA, HFIP, TFA, and reaction mixtures after 10, 20, 50 and $70 \mathrm{~min}$.

2. The reaction could proceed to first form aziridine via nitrene transfer under photoredox conditions. Then, rearrangement could occur under the same reaction conditions to form allylamine product (Figure S22). We excluded this mechanism, by subjecting the substrate $\mathbf{4 8}$ to the amination reaction conditions (Figure S23). No reaction occurred. When mixing 1 equiv of $\mathbf{4}$ with $\mathbf{4 8}$ in the same reaction conditions, no reaction occured.<smiles>[R]C/C=C/CN[R]</smiles>

Figure S22. Process via aziridination.

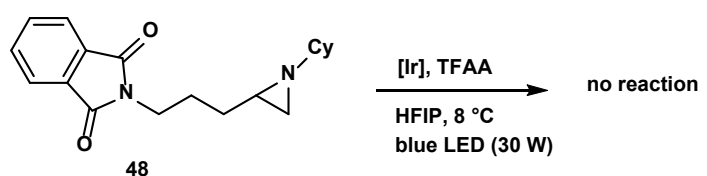<smiles>c1ccc2c(c1)Sc1ccccc1S2</smiles>

4

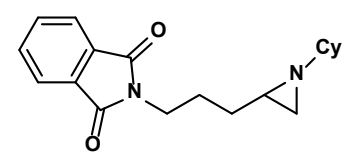

48

$\stackrel{\text { [Ir], TFAA }}{\longrightarrow}$ no reaction blue LED (30 W)

Figure S23. Reaction of $\mathbf{4 8}$ in the optimized reaction conditions. 


\section{DFT Calculation}

\section{Methods}

Density Functional Theory (DFT) calculations were performed on the Max-Planck-Institut für Kohlenforschung computer cluster using the ORCA program package (Version 4.1.x-Stable). ${ }^{7}$ Unless denoted otherwise, structural optimizations and frequency calculations to identify all of the stationary points as minima (zero imaginary frequencies) and to obtain thermal and entropic correction were performed with the B3LYP functional ${ }^{8,9}$ with D3 dispersion correction ${ }^{10}$ and Becke-Johnson damping (BJ) ${ }^{11}$ along with RI approximation, utilizing the def2/J auxiliary basis set ${ }^{12}$ and the def2-TZVPP basis set $^{13}$ on all atoms. The libint2 library was used for the computation of 2-el integrals. ${ }^{14}$ Tight SCF convergence and geometry optimization criterions were chosen. Solvent effects of HFIP were taken into account using the conductor-like polarized continuum model (CPCM, with manual input: epsilon 17.8 for dielectric constant; refrac 1.89 for refraction index). ${ }^{15}$ Molecular orbital energies were generated through the LargePrint command. Input files and images were created using Avogadro $1.2 .^{16}$
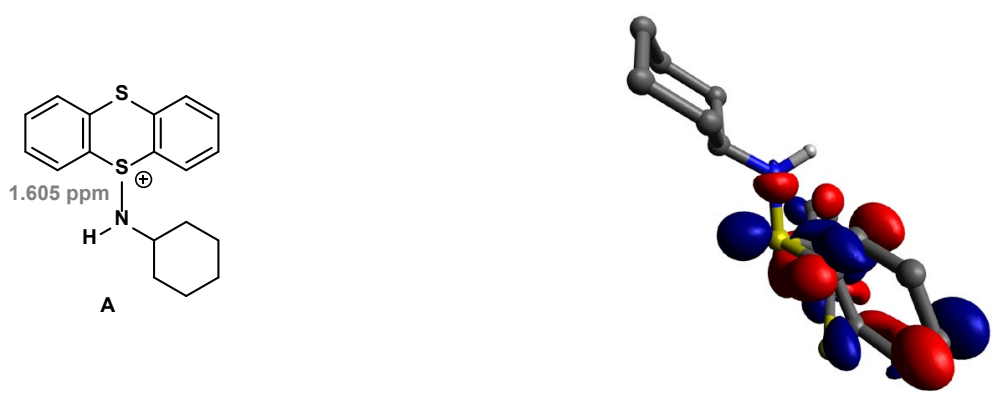

Figure S24. Visualization of the lowest unoccupied molecular orbital (iso-surface value $=0.05$ ) of the optimized aminyl thianthrenium salt $\mathbf{A}$. (N-S bond length: 1.605 ppm).

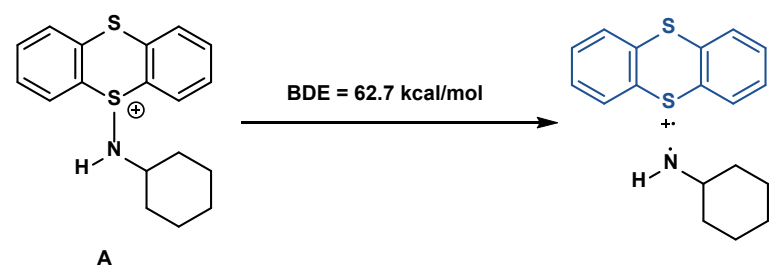

Gibb's free enthalpies were obtained by unrestricted Kohn-Sham calculations at the B3LYP level with D3 dispersion correction and Becke-Johnson damping (BJ) along with $\mathrm{RI}$ approximation, utilizing the def2/J auxiliary basis set and the def2-TZVPP basis set on all atoms. The libint2 library was used for the computation of 2-el integrals. 
Calculated Coordinates (without counterions) (B3LYP):

\section{Aminyl thianthrenium cation A}

$\begin{array}{lrrr}\text { S } & 0.476993000 & 0.260074000 & 0.957134000 \\ \mathrm{C} & 1.553587000 & -0.701554000 & 2.014134000 \\ \mathrm{C} & 1.228860000 & -0.748804000 & 3.368902000 \\ \mathrm{C} & -0.237546000 & 0.033712000 & 3.990868000 \\ \mathrm{C} & -1.409497000 & -1.489058000 & 2.765500000 \\ \mathrm{C} & -2.666974000 & -0.944552000 & 3.152418000 \\ \mathrm{C} & -3.588812000 & -1.332504000 & 2.186966000 \\ \mathrm{C} & -3.261706000 & -1.288903000 & 0.835289000 \\ \mathrm{C} & -2.009527000 & -0.838228000 & 0.434293000 \\ \mathrm{C} & 2.693104000 & -1.308723000 & 1.510063000 \\ \mathrm{C} & 3.546167000 & -1.961612000 & 2.392055000 \\ \mathrm{C} & 3.247942000 & -2.003192000 & 3.750298000 \\ \mathrm{C} & 2.089621000 & -1.410838000 & 4.240181000 \\ \mathrm{~N} & 0.731230000 & -0.194019000 & -0.562211000 \\ \mathrm{C} & 1.241983000 & 0.752080000 & -1.579785000 \\ \mathrm{C} & 0.503345000 & 0.497274000 & -2.890754000 \\ \mathrm{C} & 1.014470000 & 1.422029000 & -3.997872000 \\ \mathrm{C} & 2.528212000 & 1.299547000 & -4.175211000 \\ \mathrm{C} & 3.258349000 & 1.562373000 & -2.857793000 \\ \mathrm{C} & 2.755429000 & 0.637700000 & -1.746642000 \\ \mathrm{H} & -2.913599000 & -1.003596000 & 4.203168000 \\ \mathrm{H} & -4.563109000 & -1.683814000 & 2.496245000 \\ \mathrm{H} & -3.978707000 & -1.600768000 & 0.089453000 \\ \mathrm{H} & -1.745553000 & -0.787143000 & -0.611590000 \\ \mathrm{H} & 2.923185000 & -1.263494000 & 0.456266000 \\ \mathrm{H} & 4.442474000 & -2.432389000 & 2.014471000 \\ \mathrm{H} & 3.912983000 & -2.512125000 & 4.433981000 \\ \mathrm{H} & 1.845125000 & -1.468582000 & 5.291565000 \\ \mathrm{H} & 0.758662000 & -1.184713000 & -0.767981000 \\ \mathrm{H} & 0.991299000 & 1.750958000 & -1.217543000 \\ \mathrm{H} & 0.664078000 & -0.545537000 & -3.184185000 \\ \mathrm{H} & -0.568102000 & 0.630080000 & -2.735002000 \\ \mathrm{H} & 0.497644000 & 1.190799000 & -4.930712000 \\ \mathrm{H} & 0.764004000 & 2.457441000 & -3.746571000 \\ \mathrm{H} & 2.770771000 & 0.291941000 & -4.527383000 \\ \mathrm{H} & 2.873371000 & 1.996360000 & -4.941230000 \\ \mathrm{H} & 4.334727000 & 1.433148000 & -2.982252000 \\ \mathrm{H} & 3.097545000 & 2.602992000 & -2.557331000 \\ \mathrm{H} & 2.999724000 & -0.400818000 & -1.991539000 \\ & 3.248719000 & 0.874545000 & -0.801550000\end{array}$

\section{Thianthrenium radical cation}

$\begin{array}{lrrr}\text { S } & 0.476993000 & 0.260074000 & 0.957134000 \\ \text { C } & 1.553587000 & -0.701554000 & 2.014134000 \\ \text { C } & 1.228860000 & -0.748804000 & 3.368902000 \\ \text { S } & -0.237546000 & 0.033712000 & 3.990868000\end{array}$




$\begin{array}{lrrr}\mathrm{C} & -1.409497000 & -0.489058000 & 2.765500000 \\ \mathrm{C} & -1.106570000 & -0.436789000 & 1.406286000 \\ \mathrm{C} & -2.666974000 & -0.944552000 & 3.152418000 \\ \mathrm{C} & -3.588812000 & -1.332504000 & 2.186966000 \\ \mathrm{C} & -3.261706000 & -1.288903000 & 0.835289000 \\ \mathrm{C} & -2.009527000 & -0.838228000 & 0.434293000 \\ \mathrm{C} & 2.693104000 & -1.308723000 & 1.510063000 \\ \mathrm{C} & 3.546167000 & -1.961612000 & 2.392055000 \\ \mathrm{C} & 3.247942000 & -2.003192000 & 3.750298000 \\ \mathrm{C} & 2.089621000 & -1.410838000 & 4.240181000 \\ \mathrm{H} & -2.913599000 & -1.003596000 & 4.203168000 \\ \mathrm{H} & -4.563109000 & -1.683814000 & 2.496245000 \\ \mathrm{H} & -3.978707000 & -1.600768000 & 0.089453000 \\ \mathrm{H} & -1.745553000 & -0.787143000 & -0.611590000 \\ \mathrm{H} & 2.923185000 & -1.263494000 & 0.456266000 \\ \mathrm{H} & 4.442474000 & -2.432389000 & 2.014471000 \\ \mathrm{H} & 3.912983000 & -2.512125000 & 4.433981000 \\ \mathrm{H} & 1.845125000 & -1.468582000 & 5.291565000\end{array}$

Amine radical

$\begin{array}{lrrr}\mathrm{N} & 0.731230000 & -0.194019000 & -0.562211000 \\ \mathrm{C} & 1.241983000 & 0.752080000 & -1.579785000 \\ \mathrm{C} & 0.503345000 & 0.497274000 & -2.890754000 \\ \mathrm{C} & 1.014470000 & 1.422029000 & -3.997872000 \\ \mathrm{C} & 2.528212000 & 1.299547000 & -4.175211000 \\ \mathrm{C} & 3.258349000 & 1.562373000 & -2.857793000 \\ \mathrm{C} & 2.755429000 & 0.637700000 & -1.746642000 \\ \mathrm{H} & 0.758662000 & -1.184713000 & -0.767981000 \\ \mathrm{H} & 0.991299000 & 1.750958000 & -1.217543000 \\ \mathrm{H} & 0.664078000 & -0.545537000 & -3.184185000 \\ \mathrm{H} & -0.568102000 & 0.630080000 & -2.735002000 \\ \mathrm{H} & 0.497644000 & 1.190799000 & -4.930712000 \\ \mathrm{H} & 0.764004000 & 2.457441000 & -3.746571000 \\ \mathrm{H} & 2.770771000 & 0.291941000 & -4.527383000 \\ \mathrm{H} & 2.873371000 & 1.996360000 & -4.941230000 \\ \mathrm{H} & 4.334727000 & 1.433148000 & -2.982252000 \\ \mathrm{H} & 3.097545000 & 2.602992000 & -2.557331000 \\ \mathrm{H} & 2.999724000 & -0.400818000 & -1.991539000 \\ \mathrm{H} & 3.248719000 & 0.874545000 & -0.801550000\end{array}$




\section{X-RAY CRYSTALLOGRAPHIC ANALYSIS}

\section{X-Ray Chrystallographic Data 2 (CCDC 2006790)}

\section{Experimental}

Thianthrenylidene $t$-butylamine 2 was crystallized from a dichloromethane/hexane mixture $(3 / 1, v / v)$. The atoms are depicted with $50 \%$ probability ellipsoids. The crystallographic data are summarized in the following table.

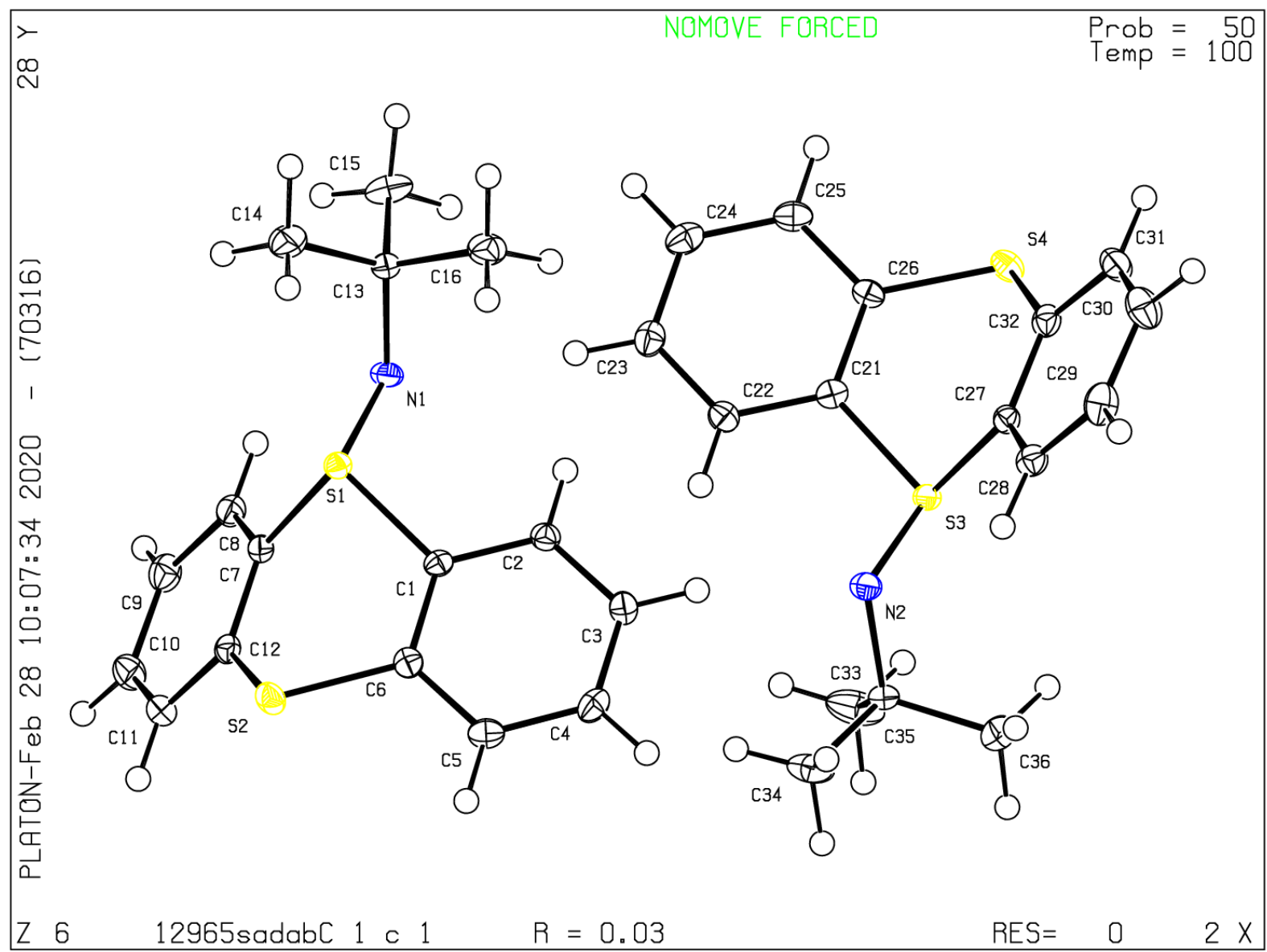

Table S10. Crystal data and structure refinement.

Empirical formula

Color

Formula weight

Temperature

Wavelength
$\mathrm{C}_{32} \mathrm{H}_{34} \mathrm{~N}_{2} \mathrm{~S}_{4}$

colorless

$574.85 \mathrm{~g} \cdot \mathrm{mol}^{-1}$

100(2) K

$0.71073 \AA$ 
Crystal system

Space group

Unit cell dimensions

Volume

Z

Density (calculated)

Absorption coefficient

$F(000)$

Crystal size

$\theta$ range for data collection

Index ranges

Reflections collected

Independent reflections

Reflections with $\mid>2 \sigma(I)$

Completeness to $\theta=25.242^{\circ}$

Absorption correction

Max. and min. transmission

Refinement method

Data / restraints / parameters

Goodness-of-fit on $\mathrm{F}^{2}$

Final $R$ indices $[I>2 \sigma(I)]$

$R$ indices (all data)

Absolute structure parameter

Largest diff. peak and hole
MONOCLINIC

Cc, (no. 9)

$$
\begin{array}{ll}
a=16.9230(6) \AA & \alpha=90^{\circ} . \\
b=10.0319(4) \AA & \beta=96.415(2)^{\circ} . \\
c=17.1857(6) \AA & \gamma=90^{\circ} .
\end{array}
$$

2899.35(19) $\AA^{3}$

4

$1.317 \mathrm{Mg} \cdot \mathrm{m}^{-3}$

$0.353 \mathrm{~mm}^{-1}$

1216 e

$0.132 \times 0.080 \times 0.061 \mathrm{~mm}^{3}$

2.364 to $30.030^{\circ}$.

$-23 \leq \mathrm{h} \leq 23,-14 \leq \mathrm{k} \leq 13,-24 \leq \mathrm{I} \leq 24$

34715

$8419\left[R_{\text {int }}=0.0368\right]$

7359

$100.0 \%$

Gaussian

0.99 and 0.97

Full-matrix least-squares on $\mathrm{F}^{2}$

8419 / 2 / 349

1.031

$\mathrm{R}_{1}=0.0339 \quad \mathrm{wR}^{2}=0.0718$

$\mathrm{R}_{1}=0.0442 \quad \mathrm{wR}^{2}=0.0757$

$-0.02(2)$

0.4 and $-0.3 e \cdot \AA^{-3}$ 
Table S11. Bond lengths $[\AA]]$ and angles $\left[^{\circ}\right]$.

\begin{tabular}{|c|c|c|c|}
\hline $\mathrm{S}(1)-\mathrm{N}(1)$ & $1.579(2)$ & $S(1)-C(1)$ & $1.796(3)$ \\
\hline$S(1)-C(7)$ & $1.790(3)$ & $S(2)-C(6)$ & $1.784(3)$ \\
\hline$S(2)-C(12)$ & $1.780(3)$ & $N(1)-C(13)$ & $1.492(3)$ \\
\hline$C(1)-C(2)$ & $1.386(4)$ & $C(1)-C(6)$ & $1.393(4)$ \\
\hline$C(2)-C(3)$ & $1.391(4)$ & $C(3)-C(4)$ & $1.394(4)$ \\
\hline$C(4)-C(5)$ & $1.392(4)$ & $C(5)-C(6)$ & $1.389(4)$ \\
\hline$C(7)-C(8)$ & $1.389(4)$ & $C(7)-C(12)$ & $1.400(4)$ \\
\hline $\mathrm{C}(8)-\mathrm{C}(9)$ & $1.386(4)$ & $C(9)-C(10)$ & $1.397(4)$ \\
\hline$C(10)-C(11)$ & $1.387(4)$ & $C(11)-C(12)$ & $1.391(4)$ \\
\hline$C(13)-C(14)$ & $1.530(4)$ & $C(13)-C(15)$ & $1.522(4)$ \\
\hline$C(13)-C(16)$ & $1.532(4)$ & $\mathrm{S}(3)-\mathrm{N}(2)$ & $1.582(2)$ \\
\hline$S(3)-C(21)$ & $1.795(3)$ & $S(3)-C(27)$ & $1.796(3)$ \\
\hline$S(4)-C(26)$ & $1.774(3)$ & $S(4)-C(32)$ & $1.780(3)$ \\
\hline$N(2)-C(33)$ & $1.504(3)$ & $C(21)-C(22)$ & $1.388(4)$ \\
\hline$C(21)-C(26)$ & $1.400(4)$ & $C(22)-C(23)$ & $1.386(4)$ \\
\hline$C(23)-C(24)$ & $1.394(4)$ & $C(24)-C(25)$ & $1.388(4)$ \\
\hline$C(25)-C(26)$ & $1.395(4)$ & $\mathrm{C}(27)-\mathrm{C}(28)$ & $1.384(4)$ \\
\hline$C(27)-C(32)$ & $1.396(4)$ & $\mathrm{C}(28)-\mathrm{C}(29)$ & $1.392(4)$ \\
\hline$C(29)-C(30)$ & $1.382(4)$ & $C(30)-C(31)$ & $1.391(4)$ \\
\hline$C(31)-C(32)$ & $1.394(4)$ & $\mathrm{C}(33)-\mathrm{C}(34)$ & $1.524(4)$ \\
\hline$C(33)-C(35)$ & $1.516(4)$ & $C(33)-C(36)$ & $1.525(4)$ \\
\hline$N(1)-S(1)-C(1)$ & $109.79(13)$ & $N(1)-S(1)-C(7)$ & $106.49(12)$ \\
\hline$C(7)-S(1)-C(1)$ & $96.03(12)$ & $C(12)-S(2)-C(6)$ & $99.51(12)$ \\
\hline $\mathrm{C}(13)-\mathrm{N}(1)-\mathrm{S}(1)$ & $114.78(17)$ & $C(2)-C(1)-S(1)$ & 119.6(2) \\
\hline$C(2)-C(1)-C(6)$ & $120.7(2)$ & $C(6)-C(1)-S(1)$ & $119.6(2)$ \\
\hline$C(1)-C(2)-C(3)$ & $119.8(2)$ & $C(2)-C(3)-C(4)$ & $119.7(3)$ \\
\hline$C(5)-C(4)-C(3)$ & $120.4(3)$ & $C(6)-C(5)-C(4)$ & $119.9(3)$ \\
\hline$C(1)-C(6)-S(2)$ & $120.2(2)$ & $C(5)-C(6)-S(2)$ & $120.3(2)$ \\
\hline$C(5)-C(6)-C(1)$ & $119.5(2)$ & $C(8)-C(7)-S(1)$ & 119.5(2) \\
\hline$C(8)-C(7)-C(12)$ & $121.3(3)$ & $C(12)-C(7)-S(1)$ & 119.1(2) \\
\hline$C(9)-C(8)-C(7)$ & $118.9(3)$ & $C(8)-C(9)-C(10)$ & $120.3(3)$ \\
\hline$C(11)-C(10)-C(9)$ & $120.4(3)$ & $C(10)-C(11)-C(12)$ & $120.0(3)$ \\
\hline
\end{tabular}




$\begin{array}{llll}\mathrm{C}(7)-\mathrm{C}(12)-\mathrm{S}(2) & 120.7(2) & \mathrm{C}(11)-\mathrm{C}(12)-\mathrm{S}(2) & 120.3(2) \\ \mathrm{C}(11)-\mathrm{C}(12)-\mathrm{C}(7) & 119.0(2) & \mathrm{N}(1)-\mathrm{C}(13)-\mathrm{C}(14) & 115.0(2) \\ \mathrm{N}(1)-\mathrm{C}(13)-\mathrm{C}(15) & 104.8(2) & \mathrm{N}(1)-\mathrm{C}(13)-\mathrm{C}(16) & 110.0(2) \\ \mathrm{C}(14)-\mathrm{C}(13)-\mathrm{C}(16) & 109.2(2) & \mathrm{C}(15)-\mathrm{C}(13)-\mathrm{C}(14) & 109.2(2) \\ \mathrm{C}(15)-\mathrm{C}(13)-\mathrm{C}(16) & 108.5(2) & \mathrm{N}(2)-\mathrm{S}(3)-\mathrm{C}(21) & 106.90(13) \\ \mathrm{N}(2)-\mathrm{S}(3)-\mathrm{C}(27) & 109.08(12) & \mathrm{C}(21)-\mathrm{S}(3)-\mathrm{C}(27) & 95.81(13) \\ \mathrm{C}(26)-\mathrm{S}(4)-\mathrm{C}(32) & 98.76(13) & \mathrm{C}(33)-\mathrm{N}(2)-\mathrm{S}(3) & 113.41(17) \\ \mathrm{C}(22)-\mathrm{C}(21)-\mathrm{S}(3) & 119.1(2) & \mathrm{C}(22)-\mathrm{C}(21)-\mathrm{C}(26) & 121.5(3) \\ \mathrm{C}(26)-\mathrm{C}(21)-\mathrm{S}(3) & 119.3(2) & \mathrm{C}(23)-\mathrm{C}(22)-\mathrm{C}(21) & 119.4(3) \\ \mathrm{C}(22)-\mathrm{C}(23)-\mathrm{C}(24) & 119.9(3) & \mathrm{C}(25)-\mathrm{C}(24)-\mathrm{C}(23) & 120.5(3) \\ \mathrm{C}(24)-\mathrm{C}(25)-\mathrm{C}(26) & 120.4(3) & \mathrm{C}(21)-\mathrm{C}(26)-\mathrm{S}(4) & 120.6(2) \\ \mathrm{C}(25)-\mathrm{C}(26)-\mathrm{S}(4) & 121.1(2) & \mathrm{C}(25)-\mathrm{C}(26)-\mathrm{C}(21) & 118.3(3) \\ \mathrm{C}(28)-\mathrm{C}(27)-\mathrm{S}(3) & 119.4(2) & \mathrm{C}(28)-\mathrm{C}(27)-\mathrm{C}(32) & 121.1(3) \\ \mathrm{C}(32)-\mathrm{C}(27)-\mathrm{S}(3) & 119.4(2) & \mathrm{C}(27)-\mathrm{C}(28)-\mathrm{C}(29) & 119.3(3) \\ \mathrm{C}(30)-\mathrm{C}(29)-\mathrm{C}(28) & 119.9(3) & \mathrm{C}(29)-\mathrm{C}(30)-\mathrm{C}(31) & 121.1(3) \\ \mathrm{C}(30)-\mathrm{C}(31)-\mathrm{C}(32) & 119.2(3) & \mathrm{C}(27)-\mathrm{C}(32)-\mathrm{S}(4) & 120.4(2) \\ \mathrm{C}(31)-\mathrm{C}(32)-\mathrm{S}(4) & 120.2(2) & \mathrm{C}(31)-\mathrm{C}(32)-\mathrm{C}(27) & 119.3(3) \\ \mathrm{N}(2)-\mathrm{C}(33)-\mathrm{C}(34) & 105.1(2) & \mathrm{N}(2)-\mathrm{C}(33)-\mathrm{C}(35) & 112.7(2) \\ \mathrm{N}(2)-\mathrm{C}(33)-\mathrm{C}(36) & 111.0(2) & \mathrm{C}(34)-\mathrm{C}(33)-\mathrm{C}(36) & 108.2(2) \\ \mathrm{C}(35)-\mathrm{C}(33)-\mathrm{C}(34) & 109.3(2) & \mathrm{C}(35)-\mathrm{C}(33)-\mathrm{C}(36) & 110.3(3) \\ & & & \end{array}$




\section{SPECTROSCOPIC DATA}

${ }^{1} \mathrm{H}$ NMR of thianthrenylidene cyclohexylamine (1)

$\mathrm{CDCl}_{3}, 25^{\circ} \mathrm{C}$

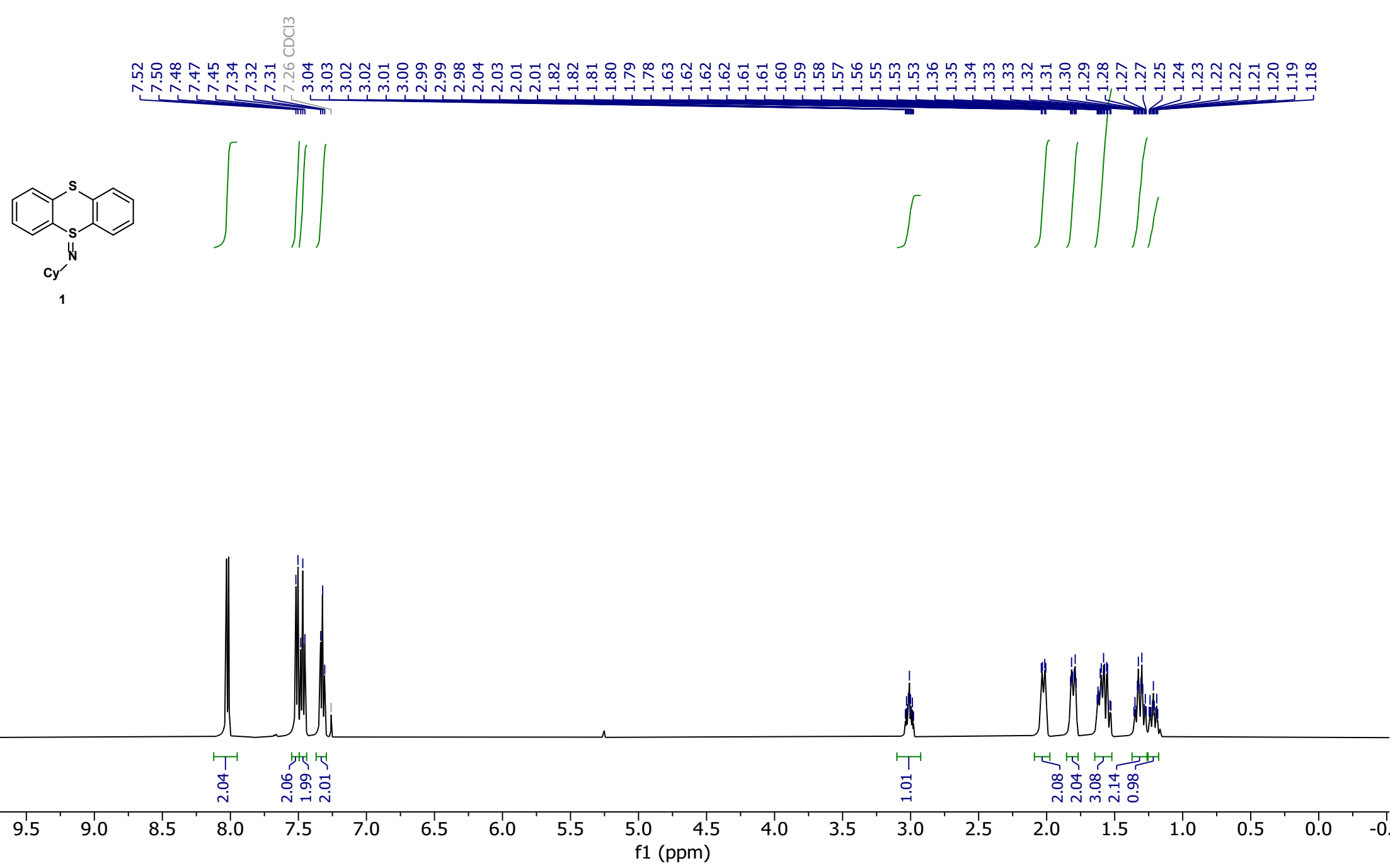


SUPPORTING INFORMATION

S75

${ }^{13} \mathrm{C}$ NMR of thianthrenylidene cyclohexylamine (1)

$\mathrm{CDCl}_{3}, 25^{\circ} \mathrm{C}$

弪

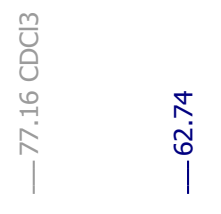

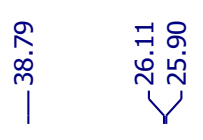
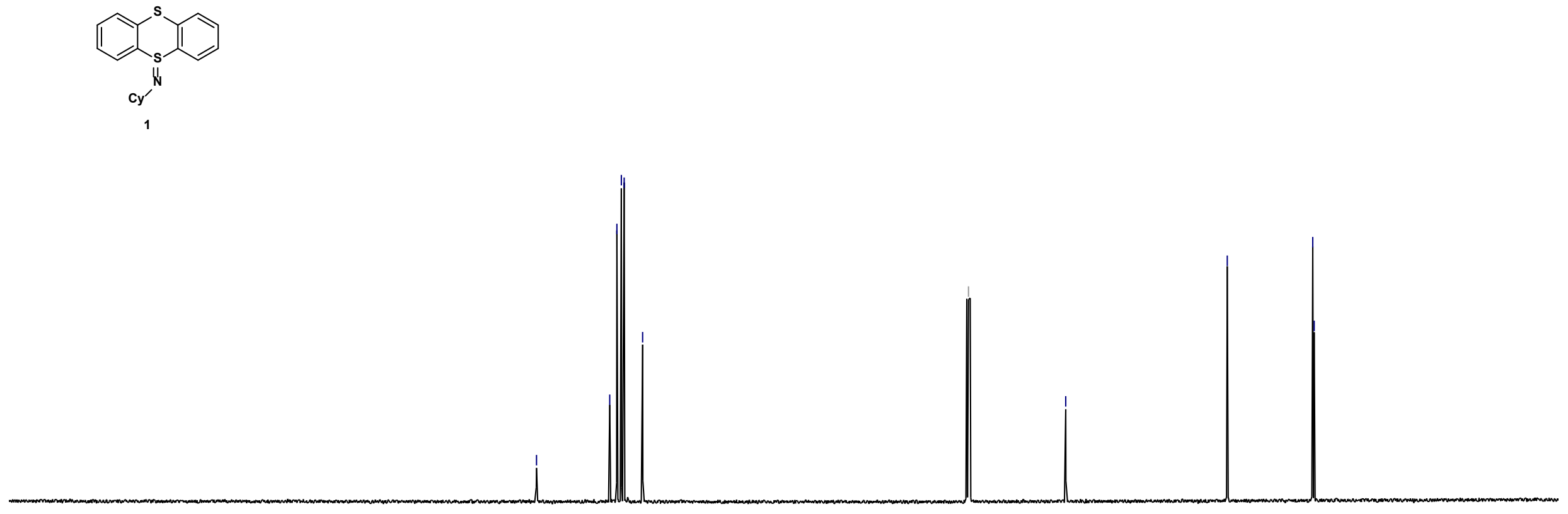

\begin{tabular}{|llllllllllllllllllllll}
\hline 210 & 200 & 190 & 180 & 170 & 160 & 150 & 140 & 130 & 120 & $\begin{array}{c}110 \\
\mathrm{f} 1(\mathrm{ppm})\end{array}$ & 90 & 80 & 70 & 60 & 50 & 40 & 30 & 20 & 10 & 0 & -1
\end{tabular}




\section{${ }^{1} \mathrm{H}$ NMR of thianthrenylidene $t$-butylamine (2)}

$\mathrm{CDCl}_{3}, 25^{\circ} \mathrm{C}$

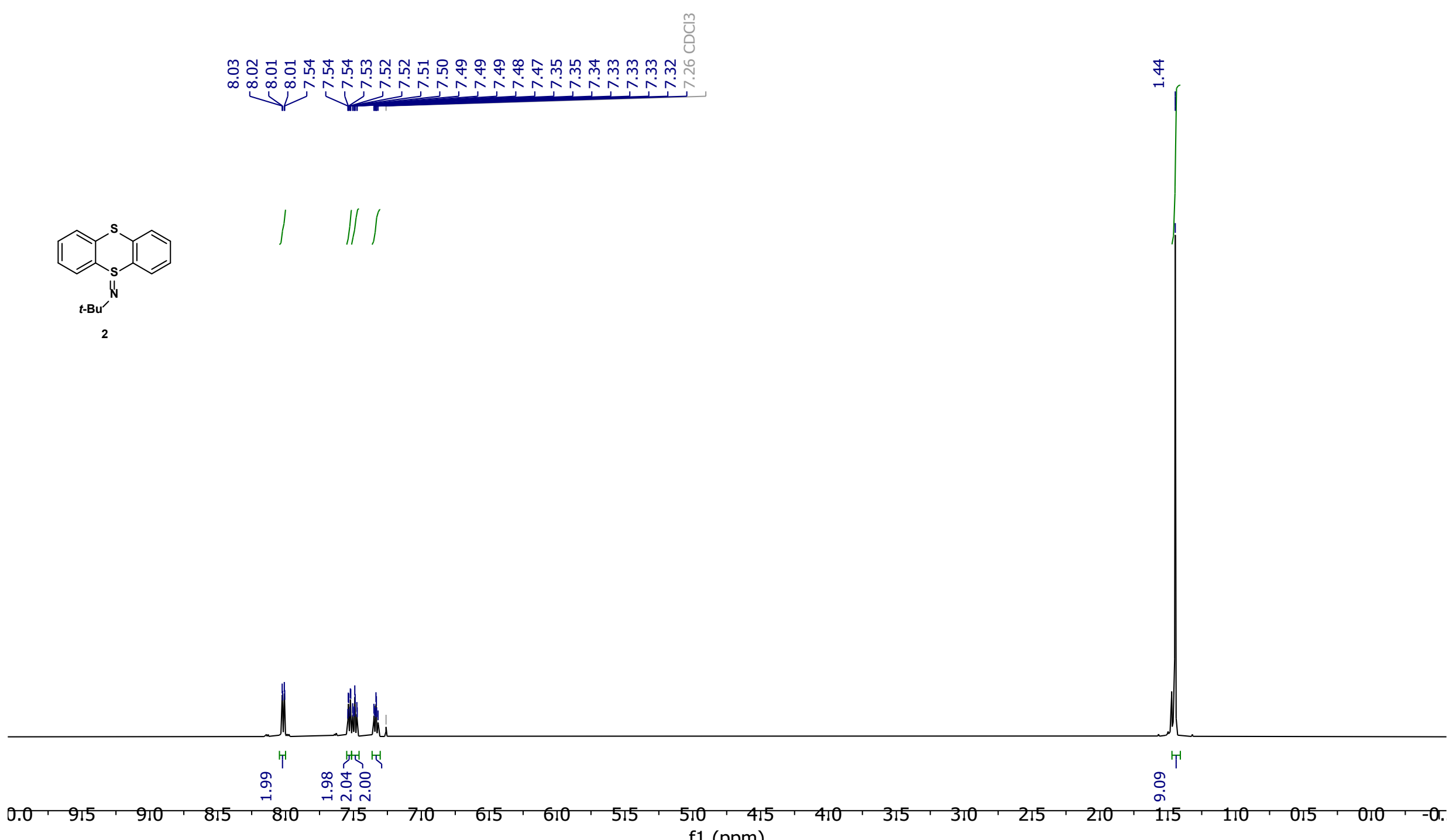

f1 (ppm) 
${ }^{13} \mathrm{C}$ NMR of thianthrenylidene $t$-butylamine (2)

$\mathrm{CDCl}_{3}, 25^{\circ} \mathrm{C}$
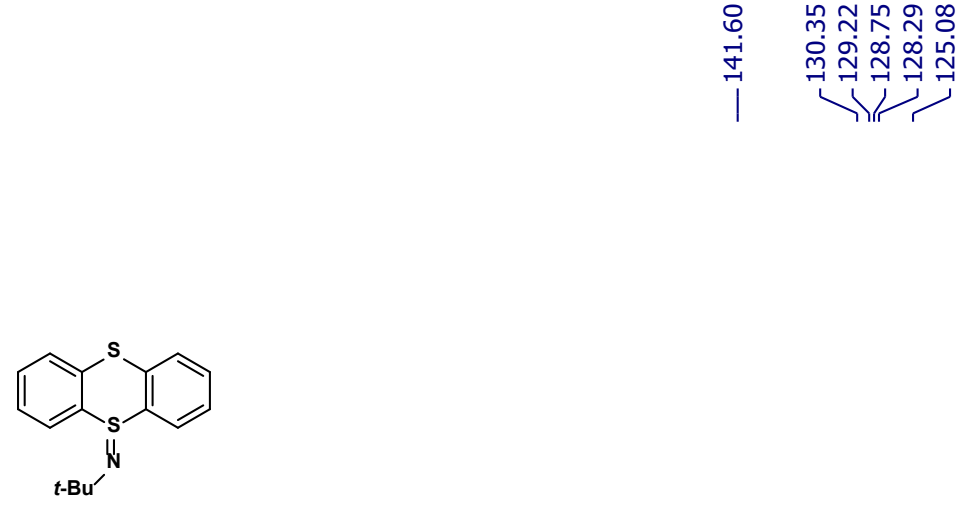

2

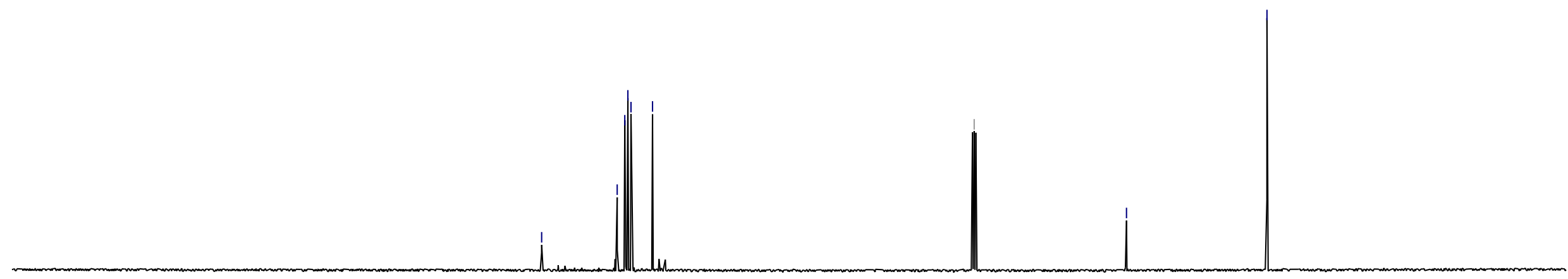

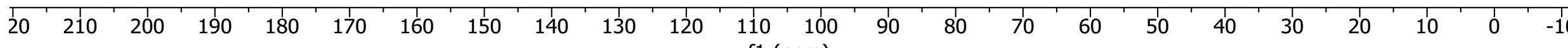
f1 (ppm) 
${ }^{1} \mathrm{H}$ NMR of thianthrenylidene $n$-propylamine (3)

$\mathrm{CDCl}_{3}, 25^{\circ} \mathrm{C}$
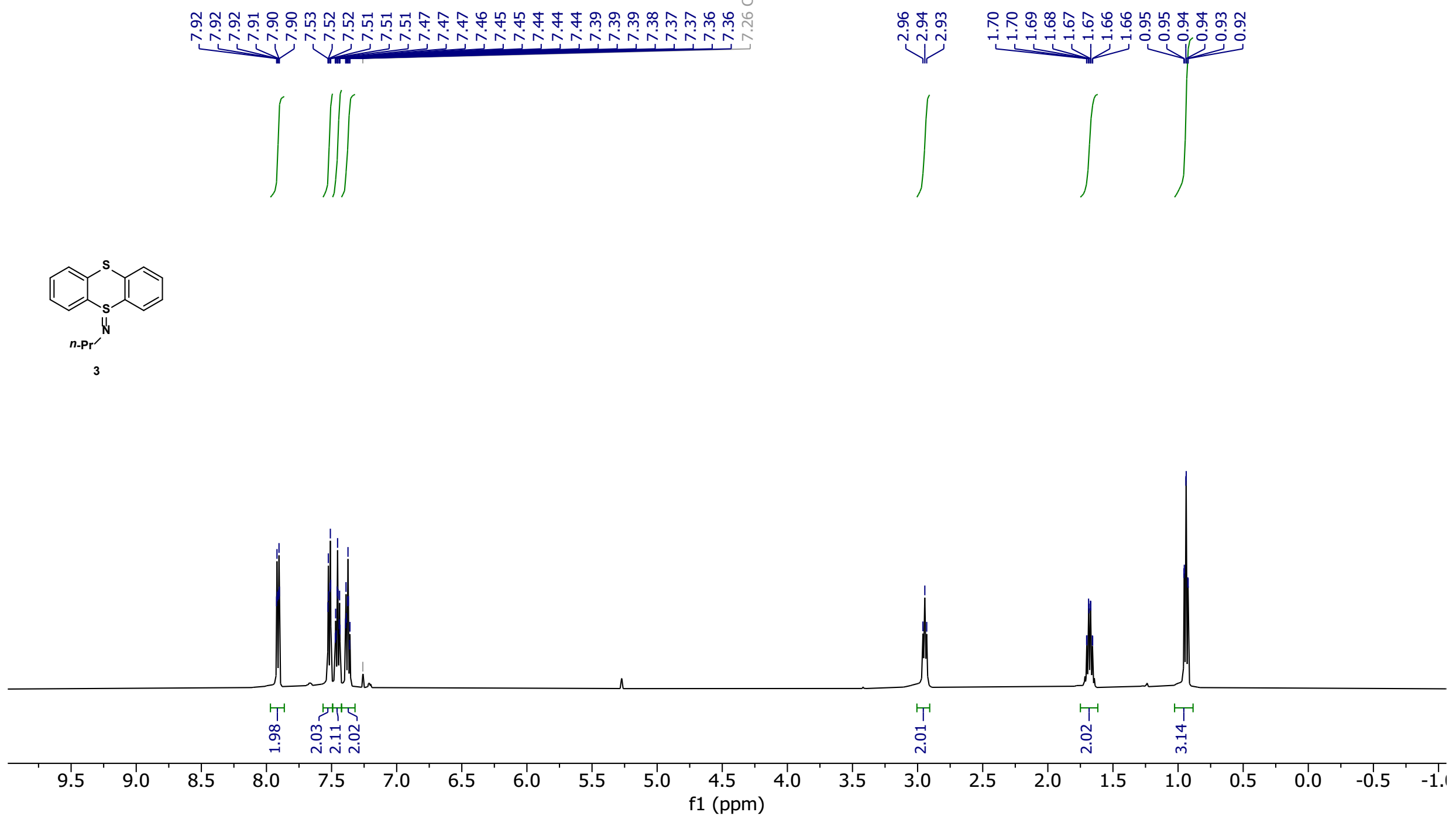
${ }^{13} \mathrm{C}$ NMR of thianthrenylidene $n$-propylamine (3)

$\mathrm{CDCl}_{3}, 25^{\circ} \mathrm{C}$

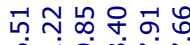

๓ं

年

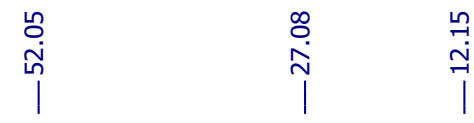
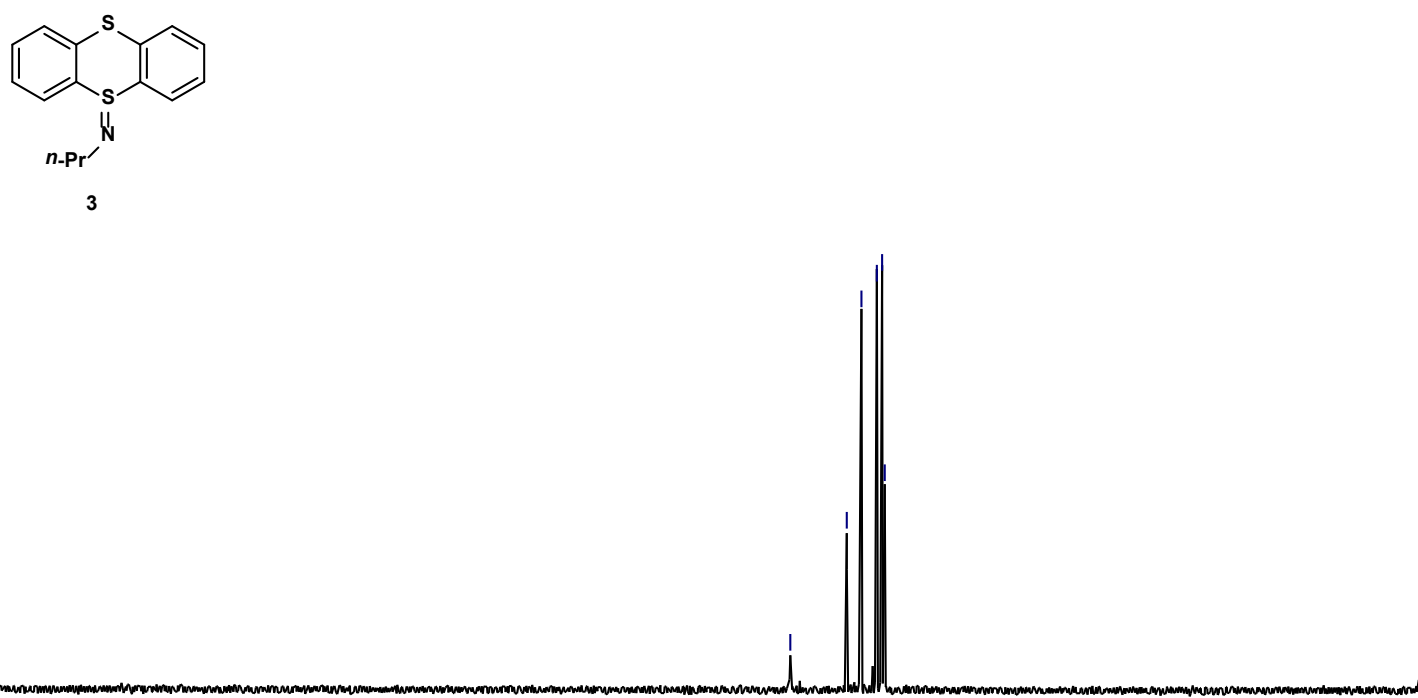

$20 \quad 210$

$\begin{array}{llllll}110 & 200 & 190 & 180 & 170 & 160\end{array}$

150

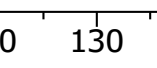

$120 \quad 110$ f1 (ppm) 
${ }^{1} \mathrm{H}$ NMR of thianthrenylidene 1-adamantylamine (4)

$\mathrm{CDCl}_{3}, 25^{\circ} \mathrm{C}$

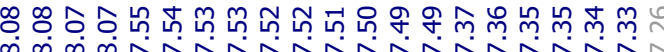
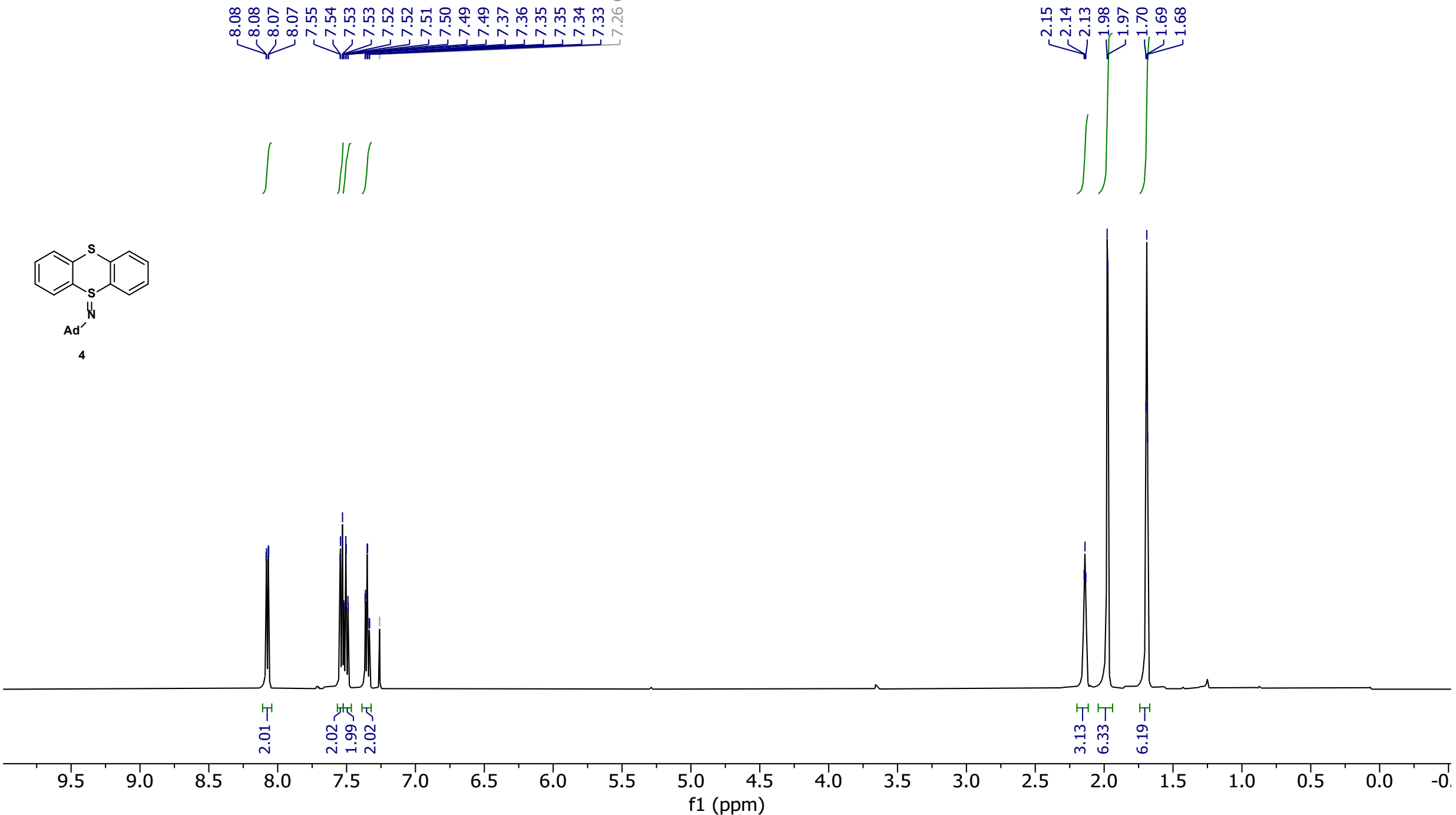
${ }^{13} \mathrm{C}$ NMR of thianthrenylidene 1-adamantylamine (4)

$\mathrm{CDCl}_{3}, 25^{\circ} \mathrm{C}$

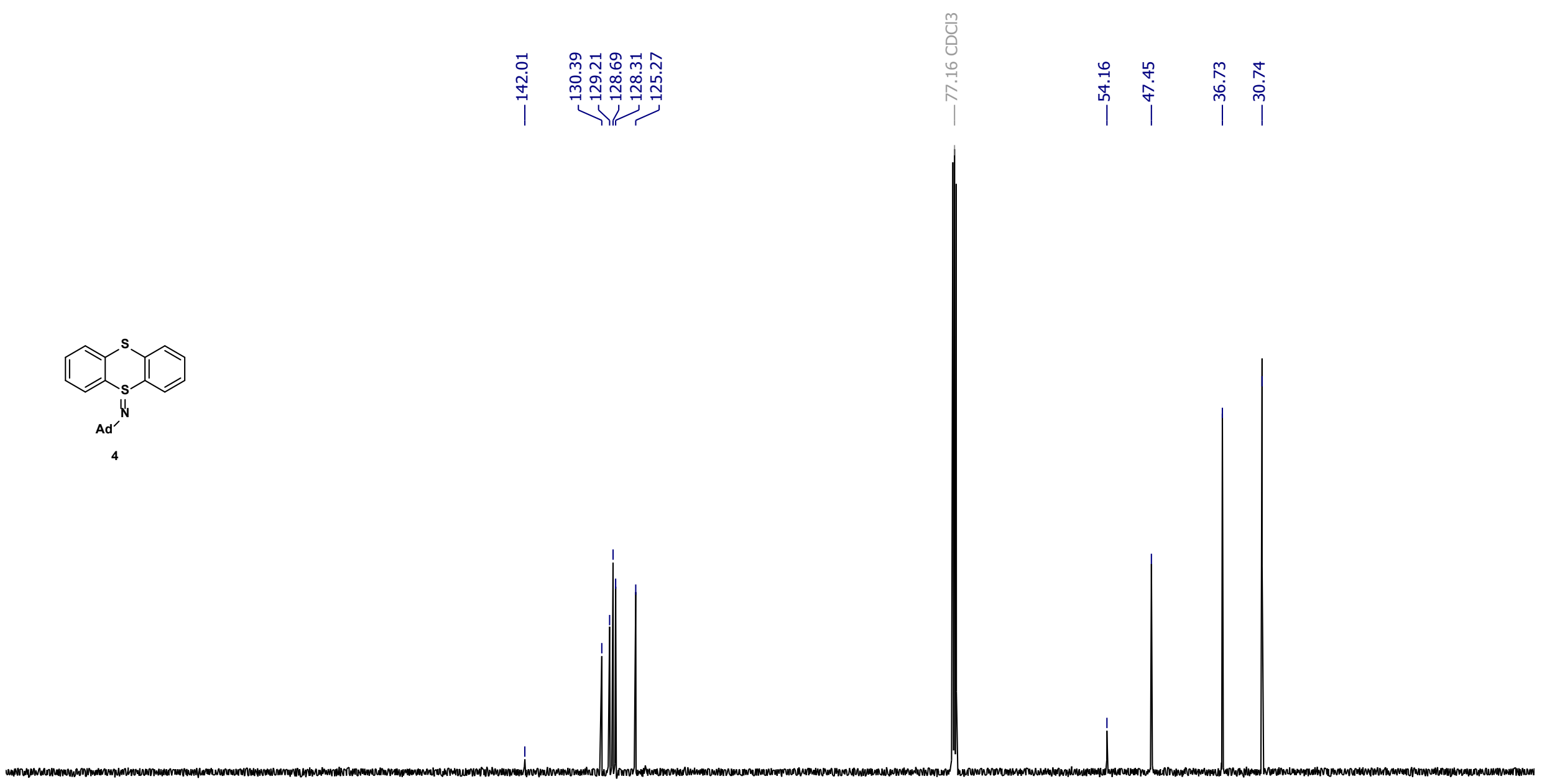

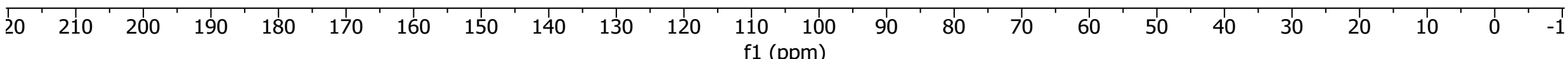


${ }^{1} \mathrm{H}$ NMR of thianthrenylidene neopentylamine (S1)

$\mathrm{CDCl}_{3}, 25^{\circ} \mathrm{C}$

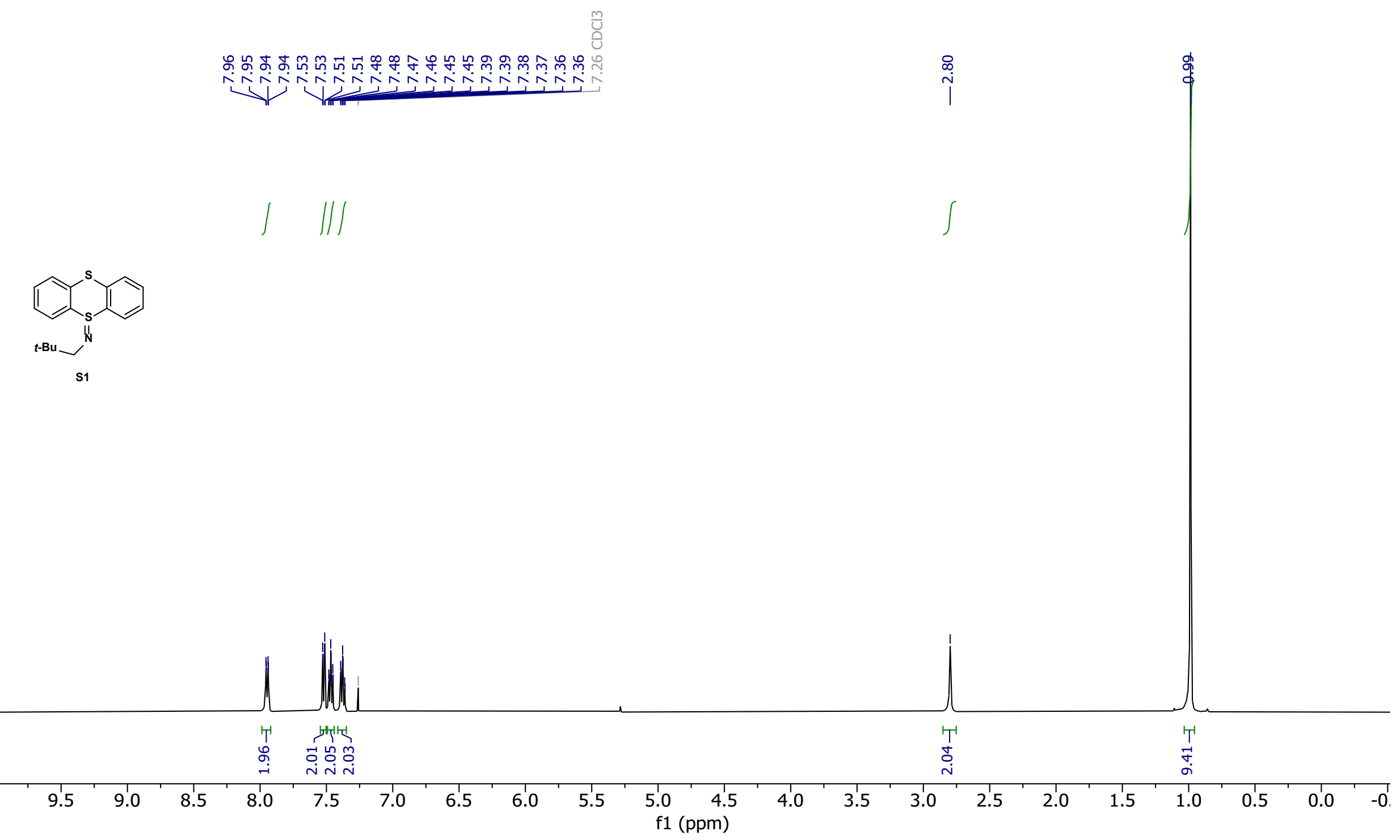


SUPPORTING INFORMATION

S83

${ }^{13} \mathrm{C}$ NMR of thianthrenylidene neopentylamine (S1)

$\mathrm{CDCl}_{3}, 25^{\circ} \mathrm{C}$

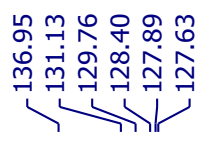

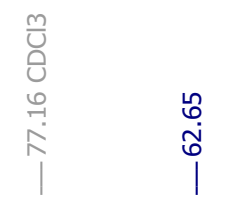

$\stackrel{\infty}{\stackrel{m}{m} \stackrel{n}{\sim}}$
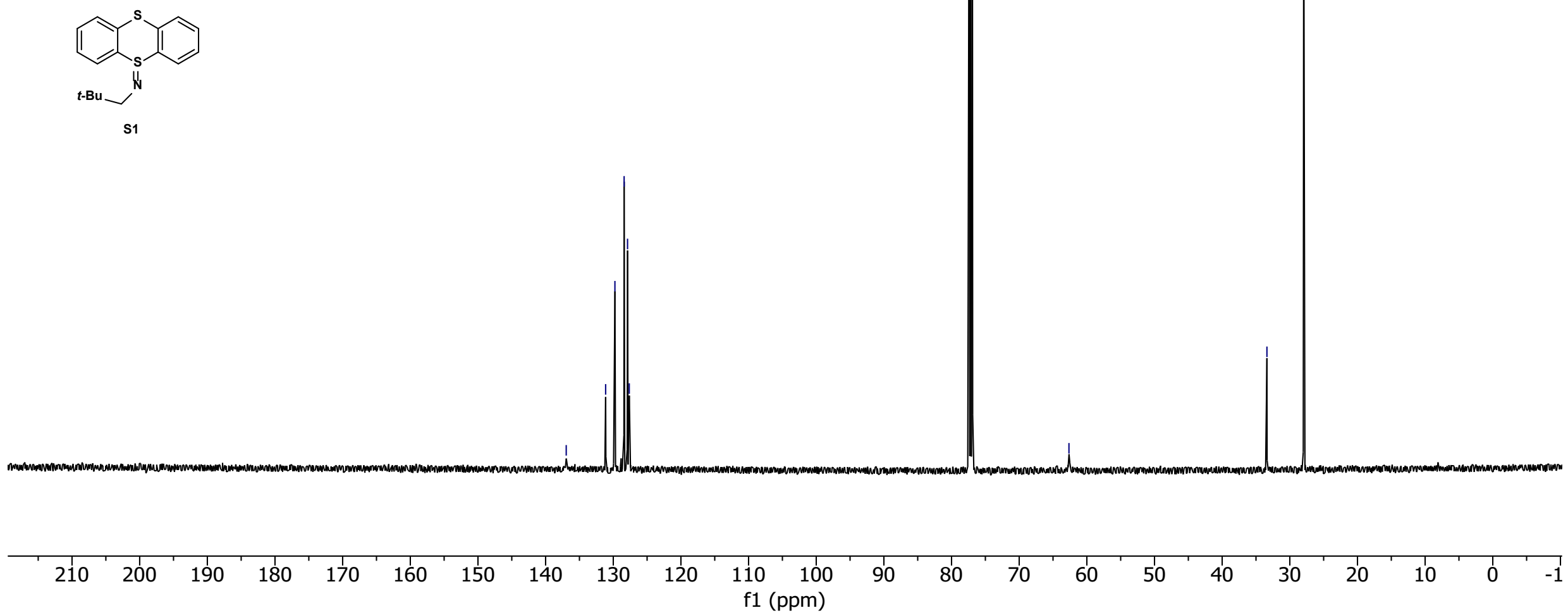
${ }^{1} \mathrm{H}$ NMR of 2-(thianthrenylidene)aminoethyl pivalate (S2)

$\mathrm{CDCl}_{3}, 25^{\circ} \mathrm{C}$

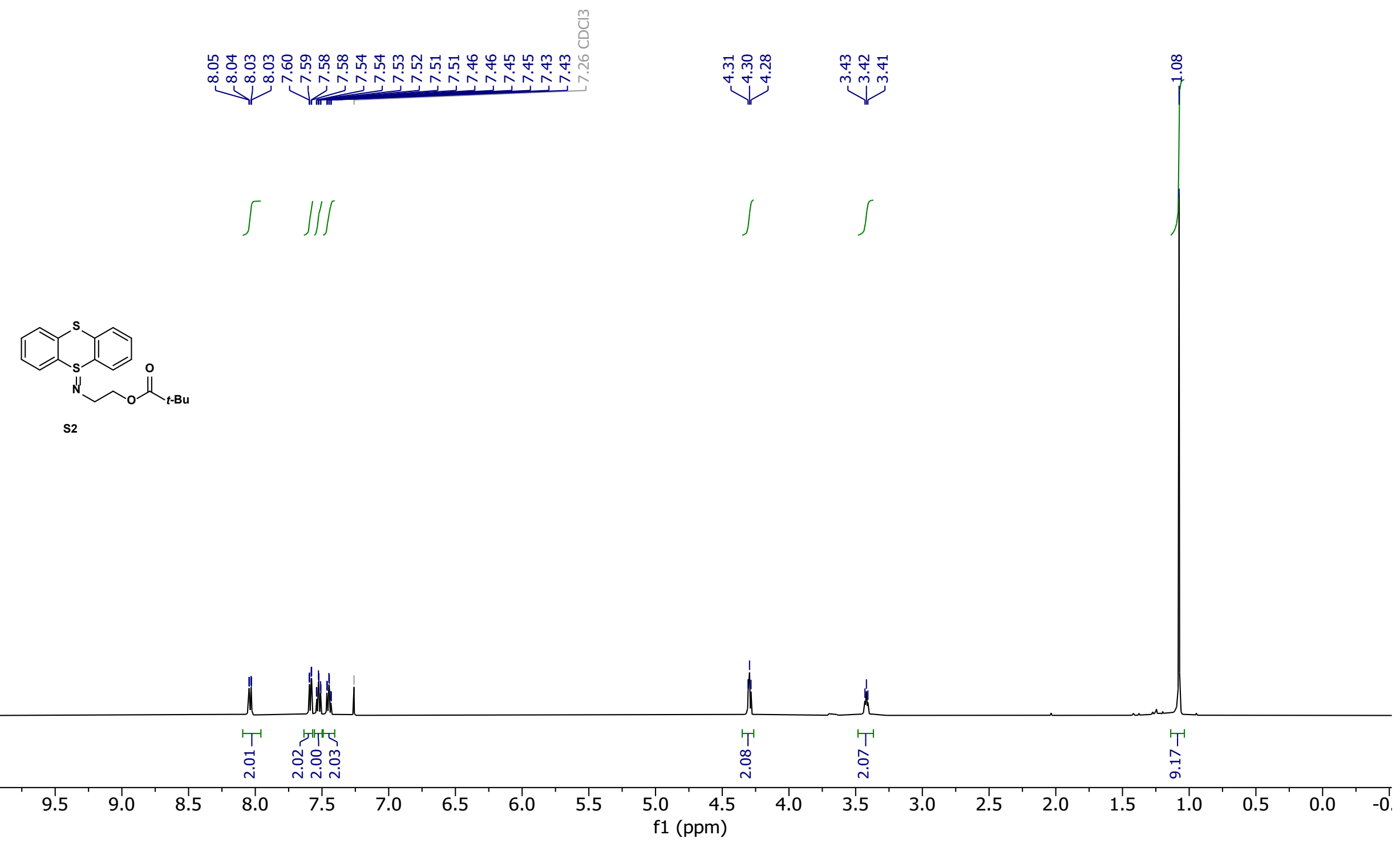


${ }^{13} \mathrm{C}$ NMR of 2-(thianthrenylidene)aminoethyl pivalate (S2)

$\mathrm{CDCl}_{3}, 25{ }^{\circ} \mathrm{C}$

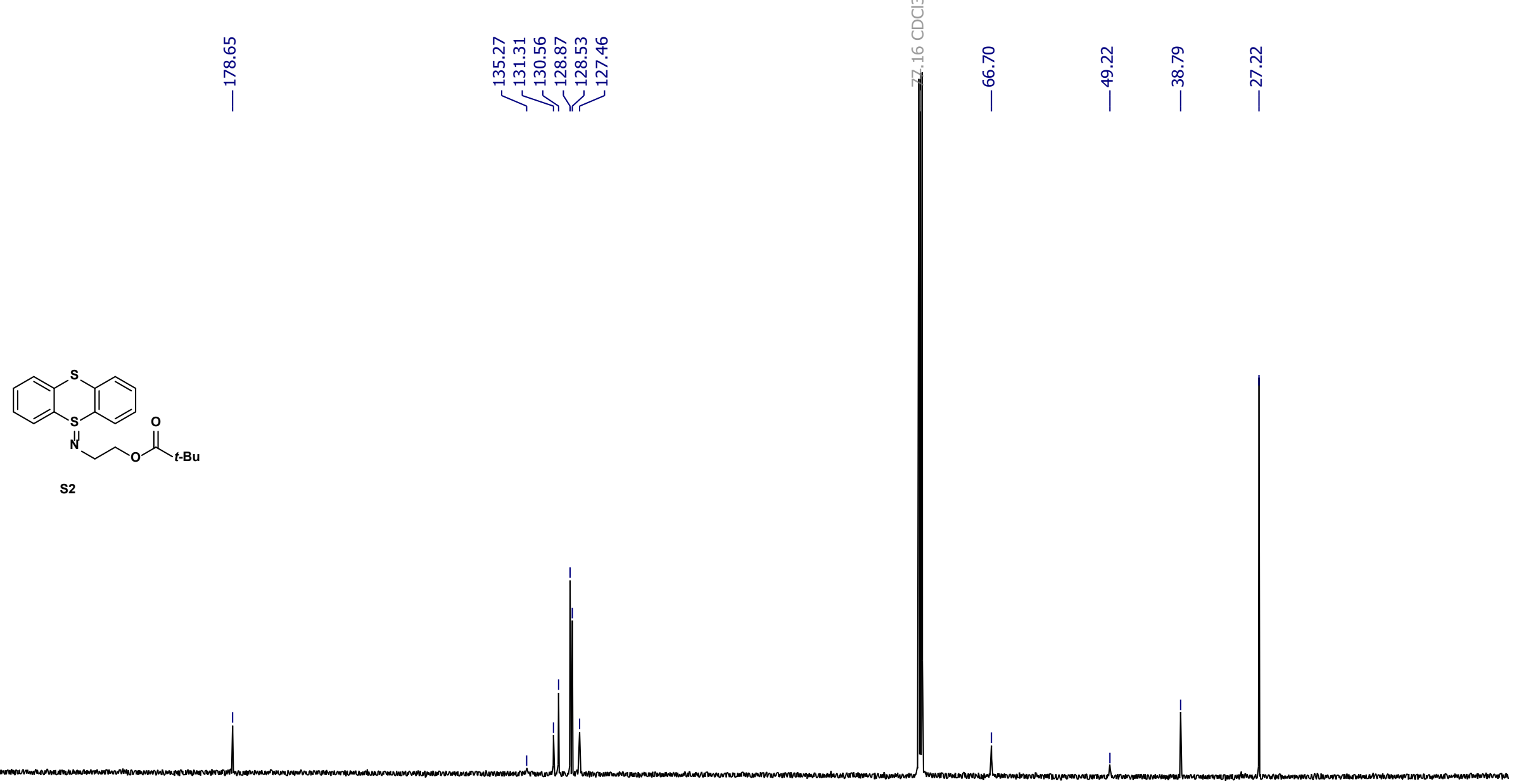

$\begin{array}{llllllllllllllllllllllllllll}20 & 210 & 200 & 190 & 180 & 170 & 160 & 150 & 140 & 130 & 120 & \begin{array}{c}110 \\ \mathrm{f}(\mathrm{ppm})\end{array} & 90 & 80 & 70 & 60 & 50 & 40 & 30 & 20 & 10 & 0 & 1\end{array}$ 
${ }^{1} \mathrm{H}$ NMR of ethyl 2-(thianthrenylidene)amino-2-methylpropanoate (S3)

$\mathrm{CDCl}_{3}, 25^{\circ} \mathrm{C}$

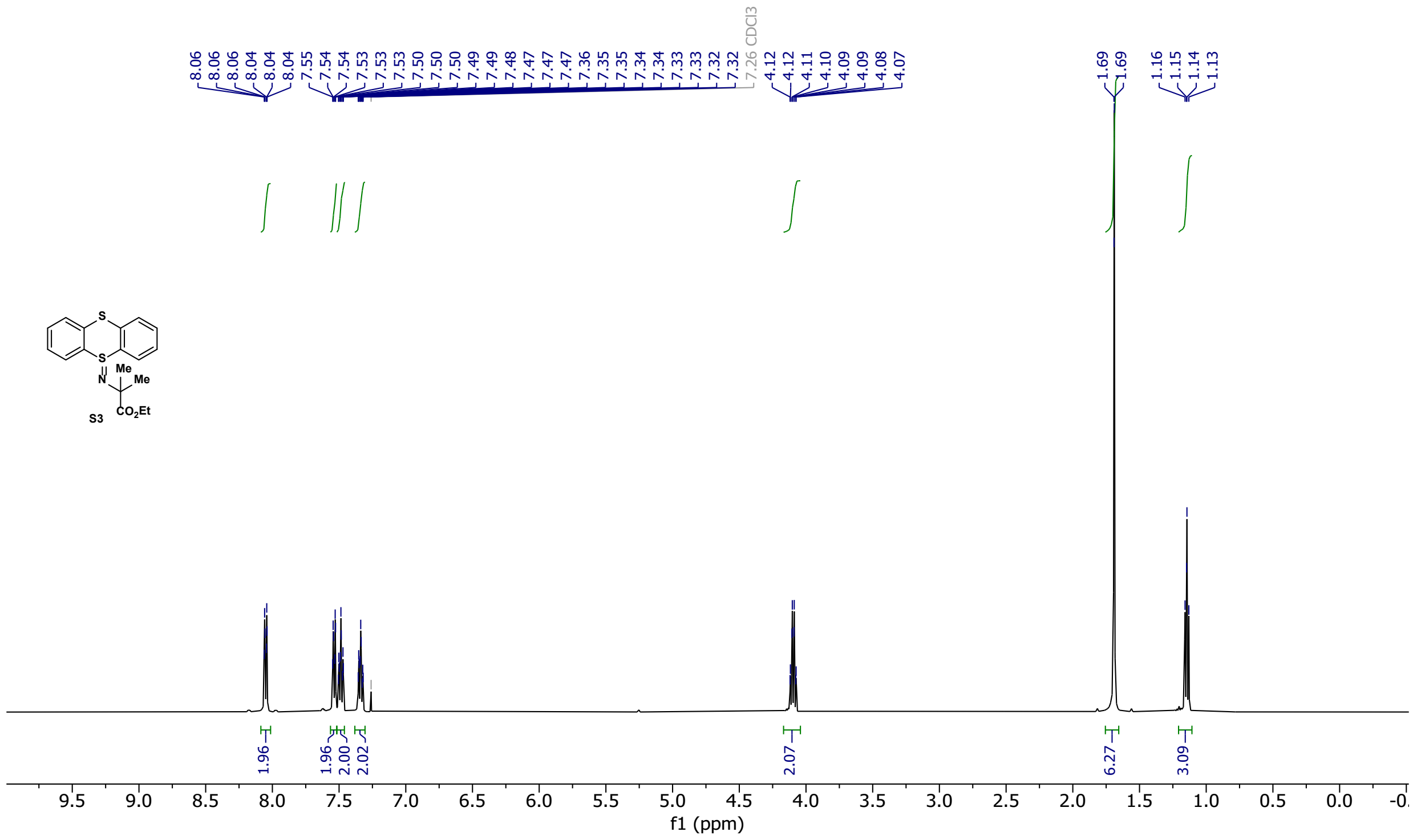


${ }^{13} \mathrm{C}$ NMR of ethyl 2-(thianthrenylidene)amino-2-methylpropanoate (S3)

$\mathrm{CDCl}_{3}, 25^{\circ} \mathrm{C}$

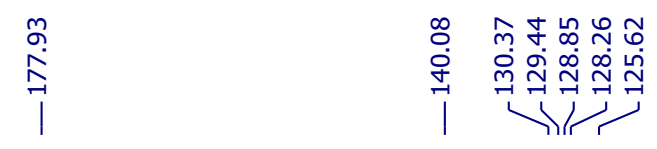

象

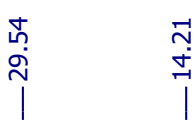

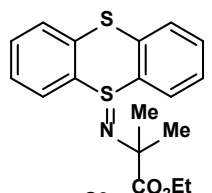

$\mathrm{S3}_{3} \mathrm{CO}_{2} \mathrm{Et}$

$\begin{array}{rllllllllllll}20 & 210 & 200 & 190 & 180 & 170 & 160 & 150 & 140 & 130 & 120 & 110 & 100 \\ \mathrm{f} 1(\mathrm{ppm})\end{array}$ 
${ }^{1} \mathrm{H}$ NMR of thianthrenylidene $\alpha$-methylbenzylamine (S4)

$\mathrm{CDCl}_{3}, 25^{\circ} \mathrm{C}$

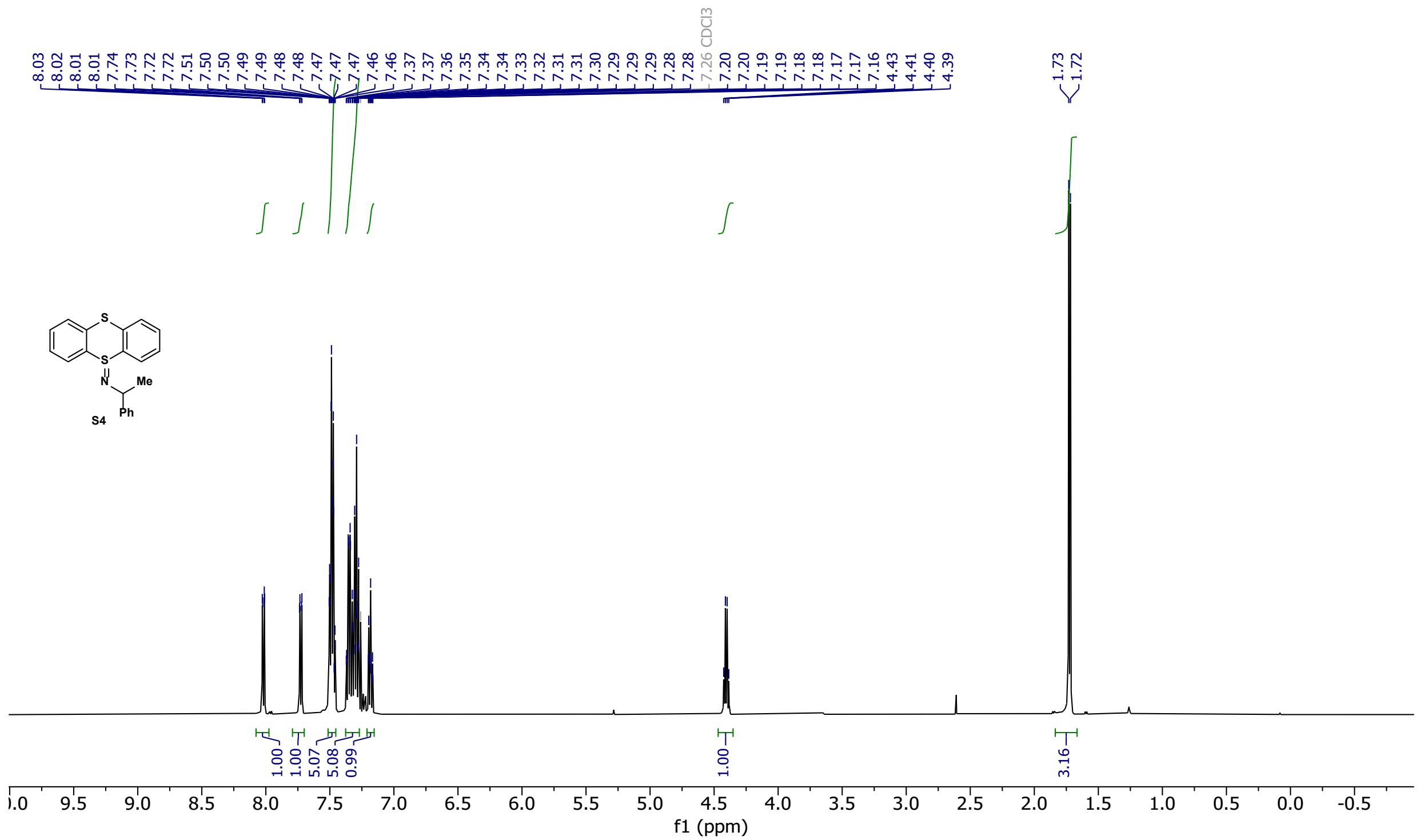


${ }^{13} \mathrm{C}$ NMR of thianthrenylidene $\alpha-$ methylbenzylamine (S4)

$\mathrm{CDCl}_{3}, 25^{\circ} \mathrm{C}$
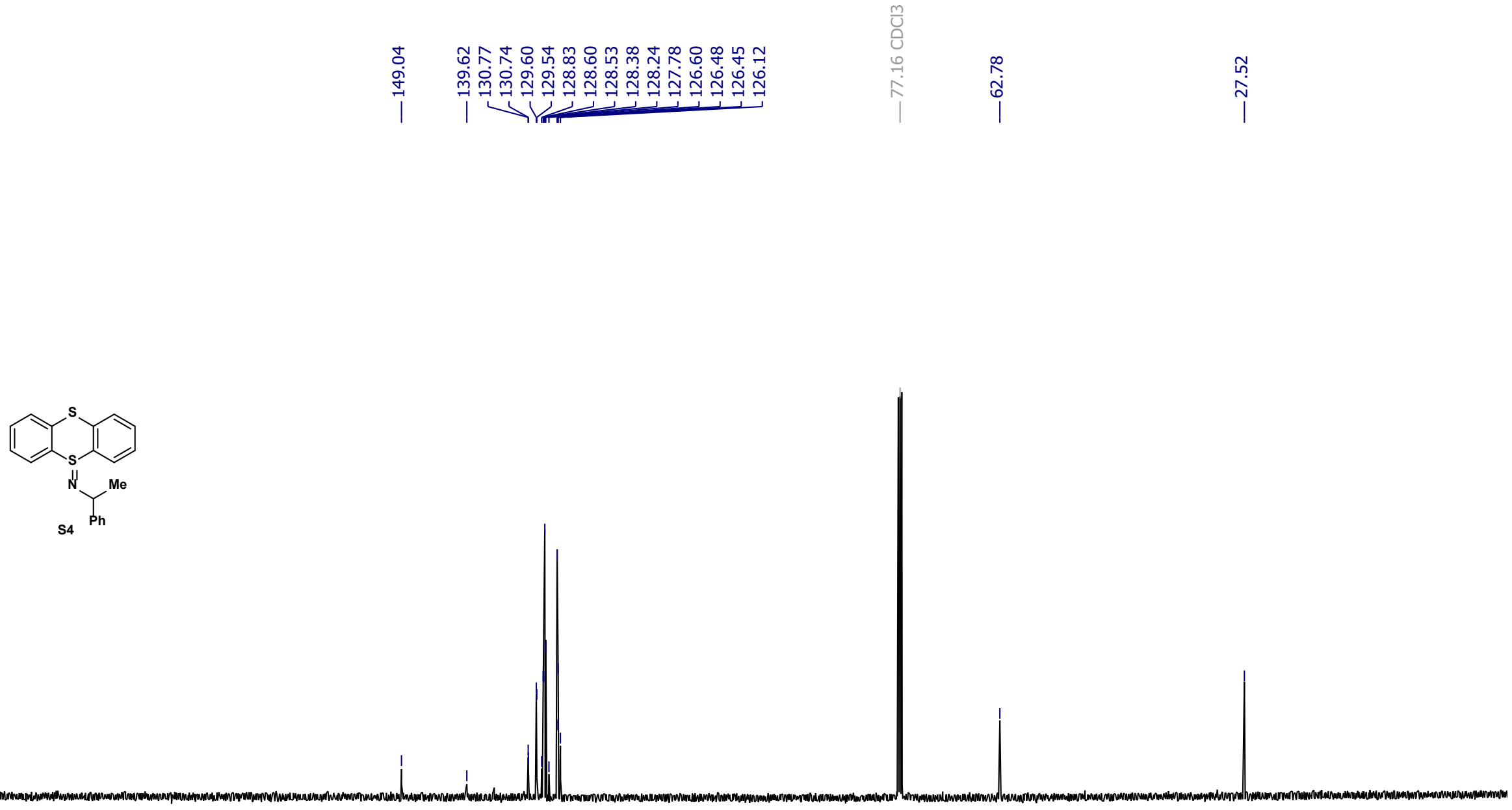

$20 \quad 210$

$200 \quad 190 \quad 180$

$170 \quad 160$

$150 \quad 140$

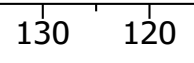

$90 \quad 80$

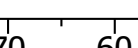




\section{${ }^{1} \mathrm{H}$ NMR of 3-(thianthrenylidene)aminopropan-1-ol (S5)}

$\mathrm{CDCl}_{3}, 25^{\circ} \mathrm{C}$
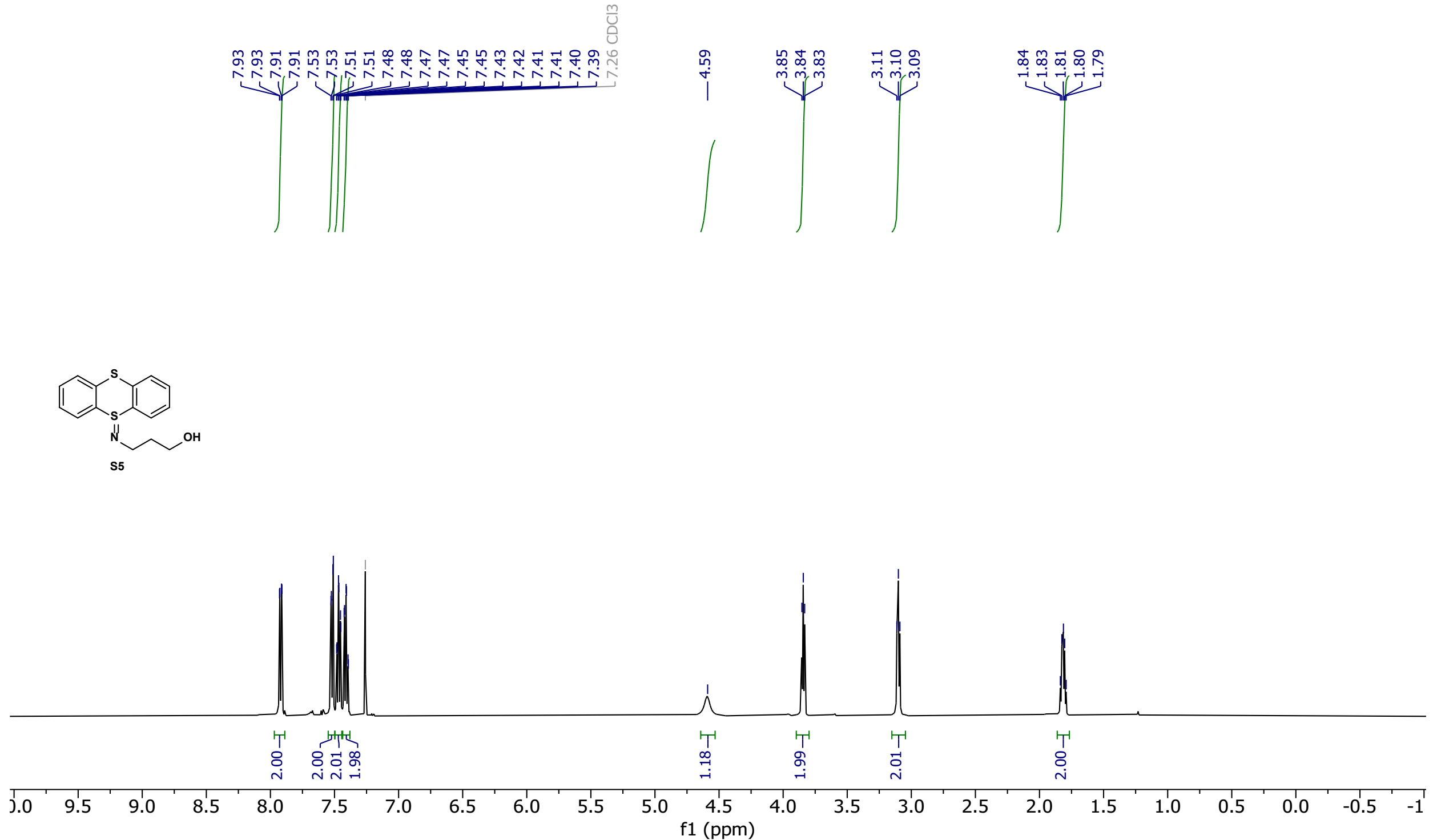
${ }^{13} \mathrm{C}$ NMR of 3-(thianthrenylidene)aminopropan-1-ol (S5)

$\mathrm{CDCl}_{3}, 25^{\circ} \mathrm{C}$

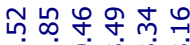

लَ̣

in

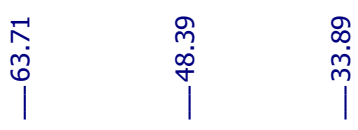

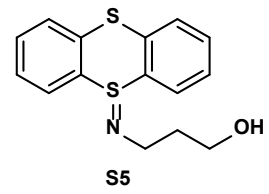

s5

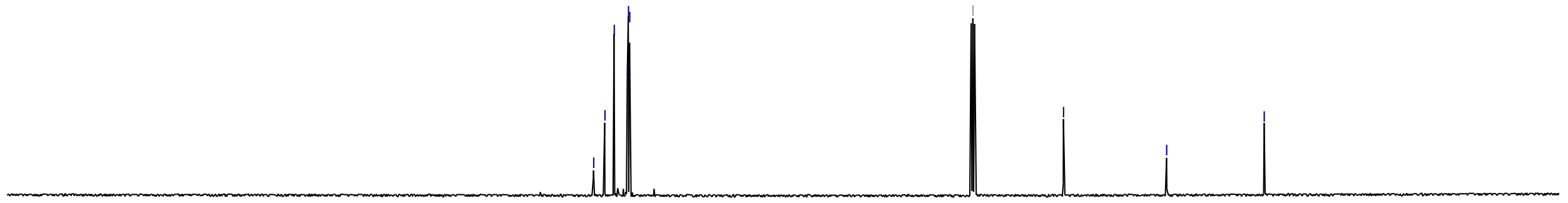


${ }^{1} \mathrm{H}$ NMR of (Z)-N-Cyclohexyl-2-cyclooctenamine (5)

$\mathrm{CDCl}_{3}, 25^{\circ} \mathrm{C}$
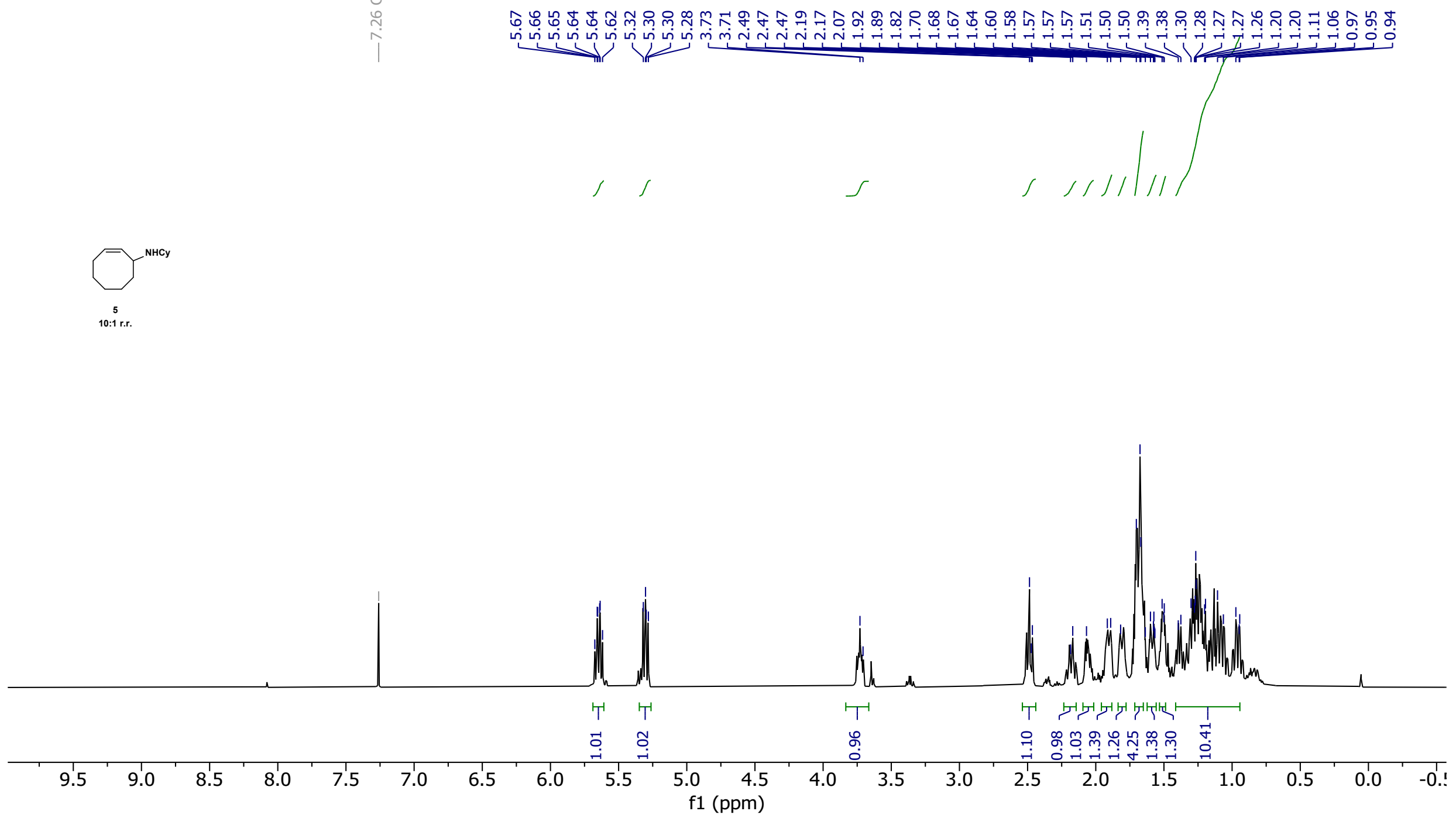
${ }^{13} \mathrm{C}$ NMR of (Z)-N-Cyclohexyl-2-cyclooctenamine (5)

$\mathrm{CDCl}_{3}, 25{ }^{\circ} \mathrm{C}$
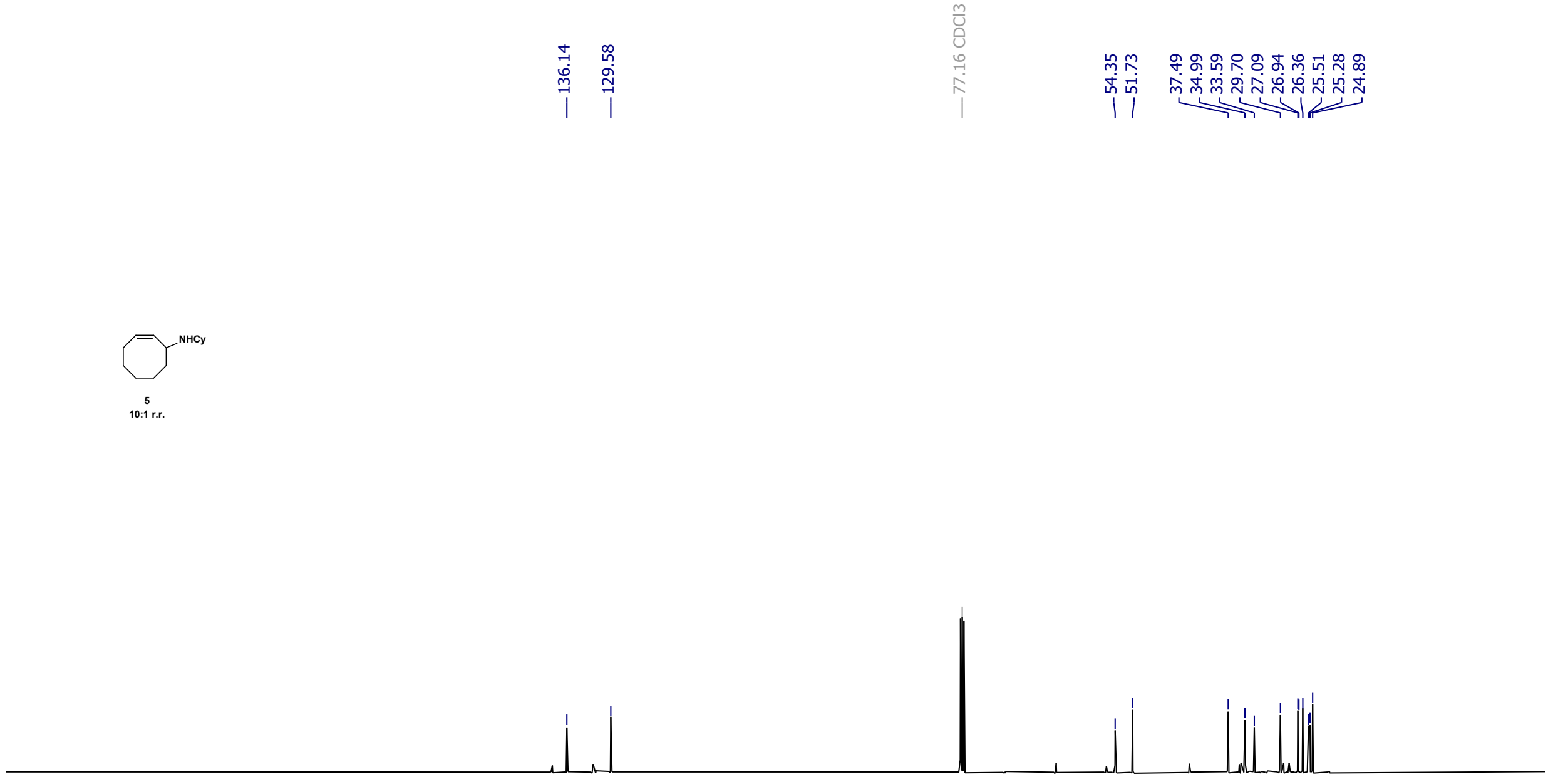

$\begin{array}{llllllllllll}210 & 200 & 190 & 180 & 170 & 160 & 150 & 140 & 130 & 120 & \begin{array}{c}110 \\ \mathrm{f} 1(\mathrm{ppm})\end{array}\end{array}$ 
${ }^{1} \mathrm{H}$ NMR of (E)-N-(4-phenyl-2-butenyl)cyclohexanamine (6)

$\mathrm{CDCl}_{3}, 25^{\circ} \mathrm{C}$

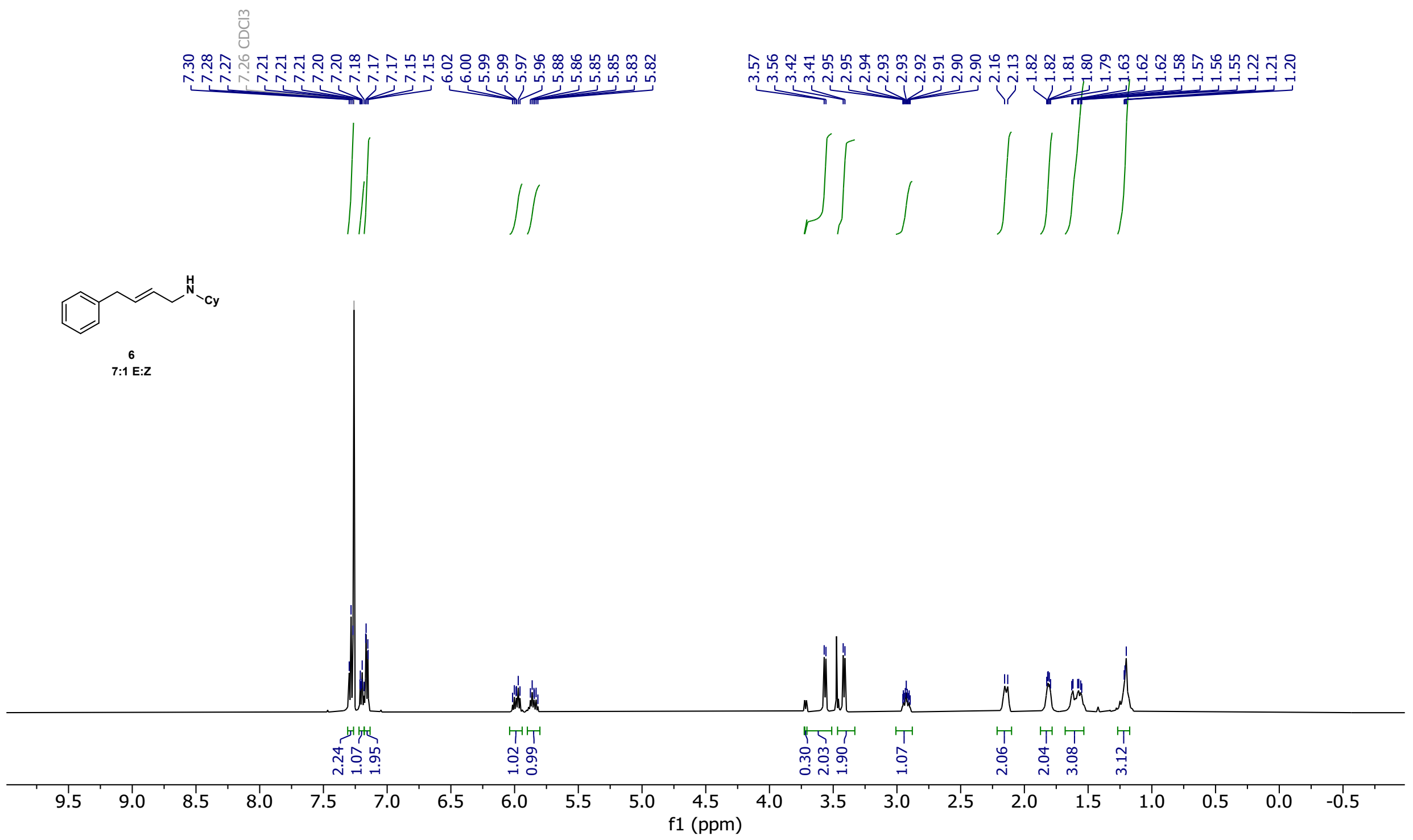


${ }^{13} \mathrm{C}$ NMR of (E)-N-(4-phenyl-2-butenyl)cyclohexanamine (6)

$\mathrm{CDCl}_{3}, 25^{\circ} \mathrm{C}$
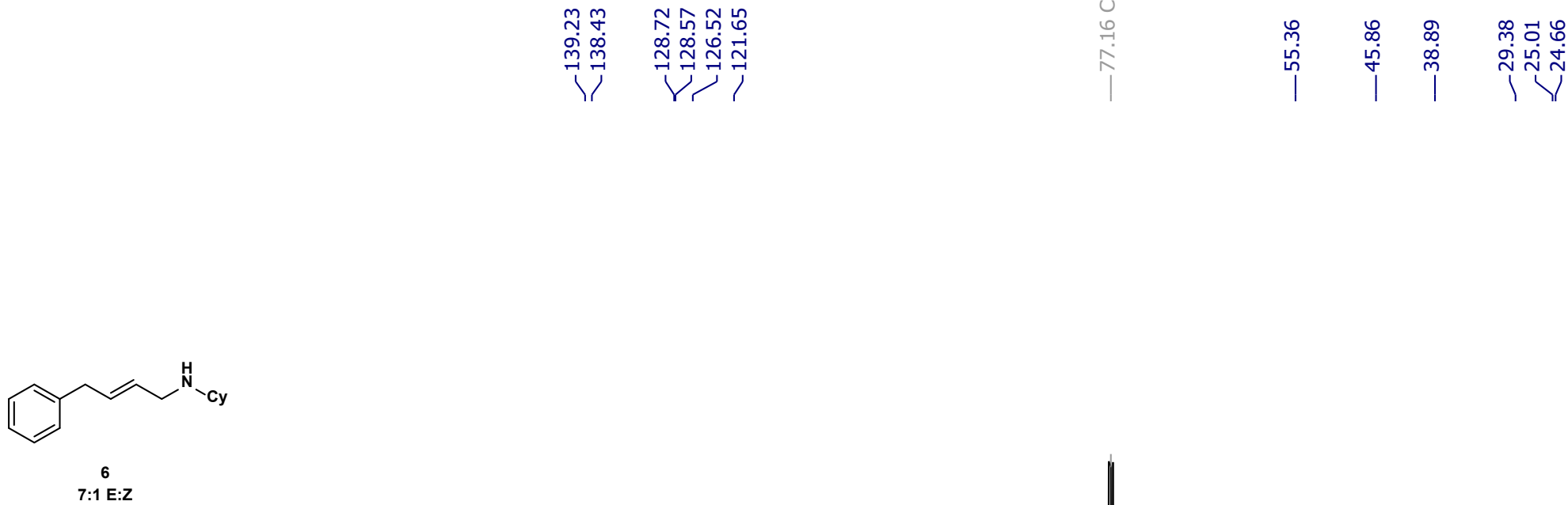

6
$7: 1 \mathrm{E}: 2$
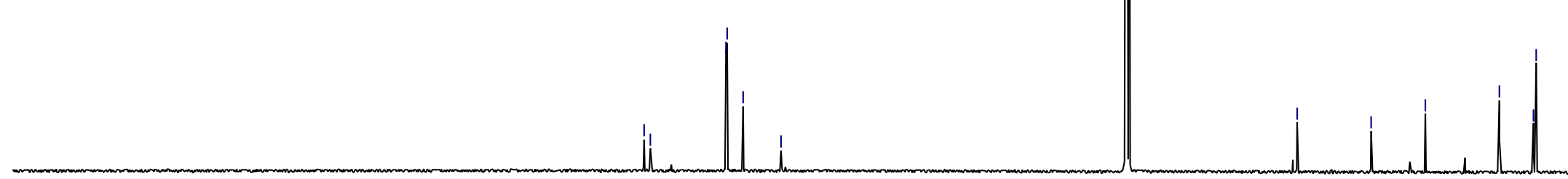

$\begin{array}{lllllllllllll}20 & 210 & 200 & 190 & 180 & 170 & 160 & 150 & 140 & 130 & 120 & 110 & 100\end{array}$ 


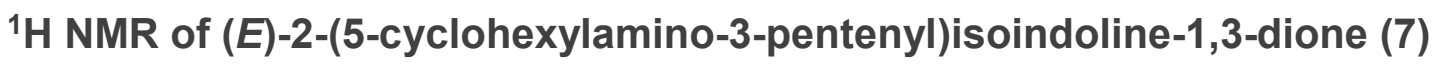

$\mathrm{CDCl}_{3}, 25^{\circ} \mathrm{C}$
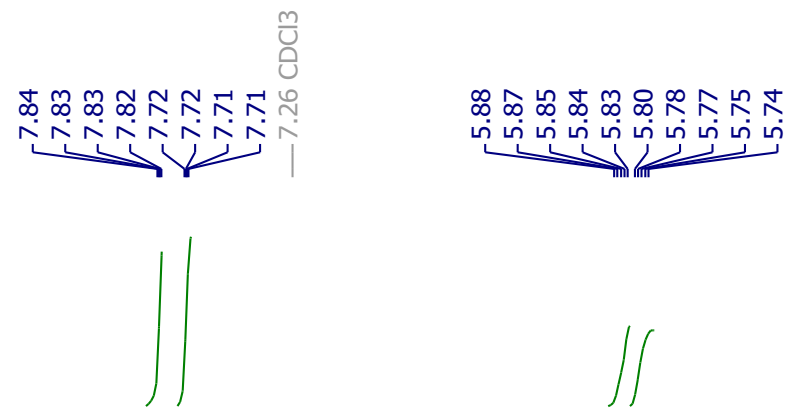

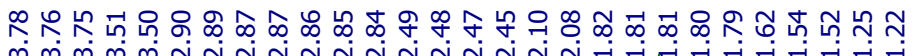
$\underbrace{}_{0}$<smiles>C=CC=C</smiles>
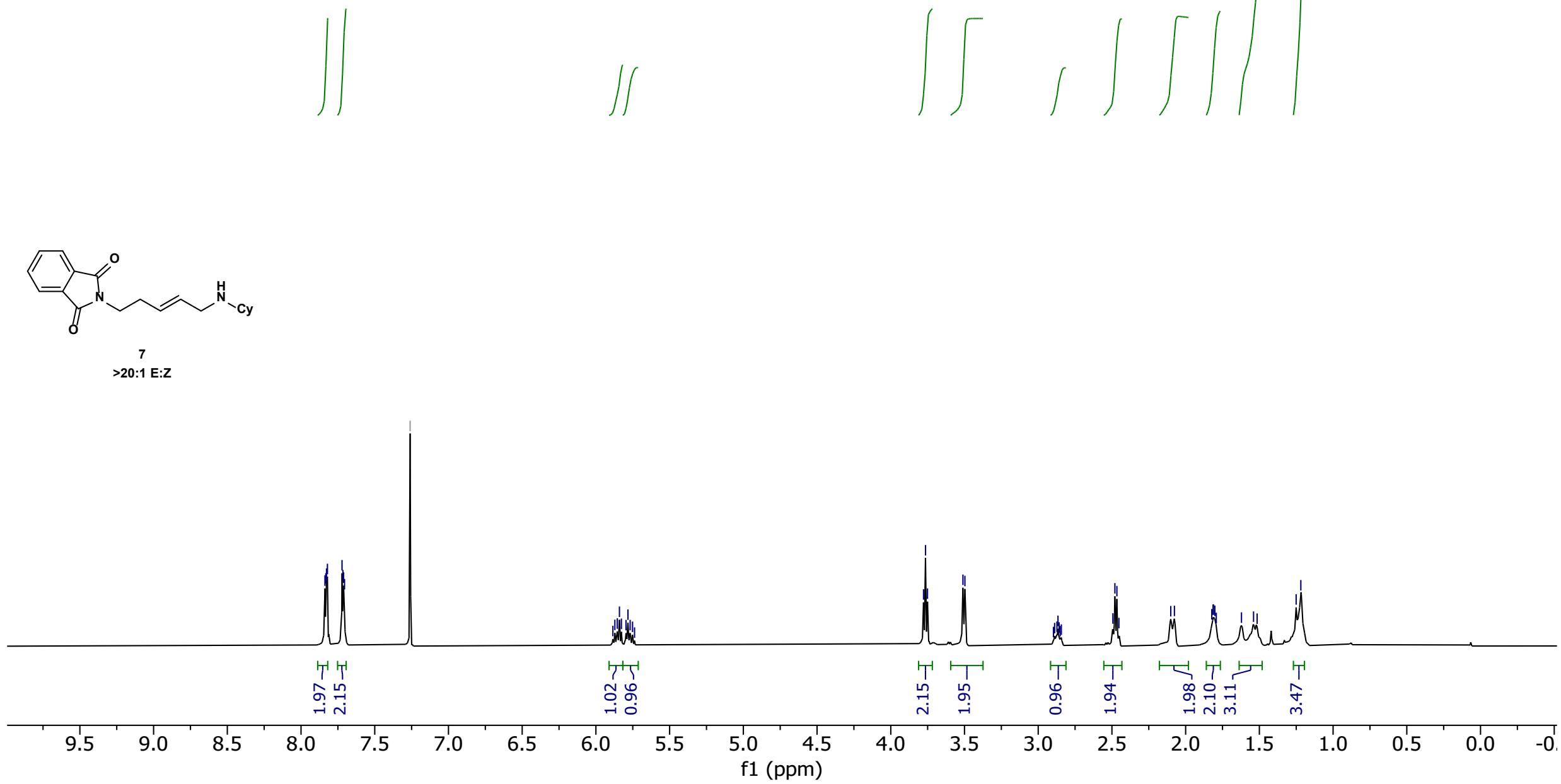
${ }^{13} \mathrm{C}$ NMR of (E)-2-(5-cyclohexylamino-3-pentenyl)isoindoline-1,3-dione (7)

$\mathrm{CDCl}_{3}, 25^{\circ} \mathrm{C}$

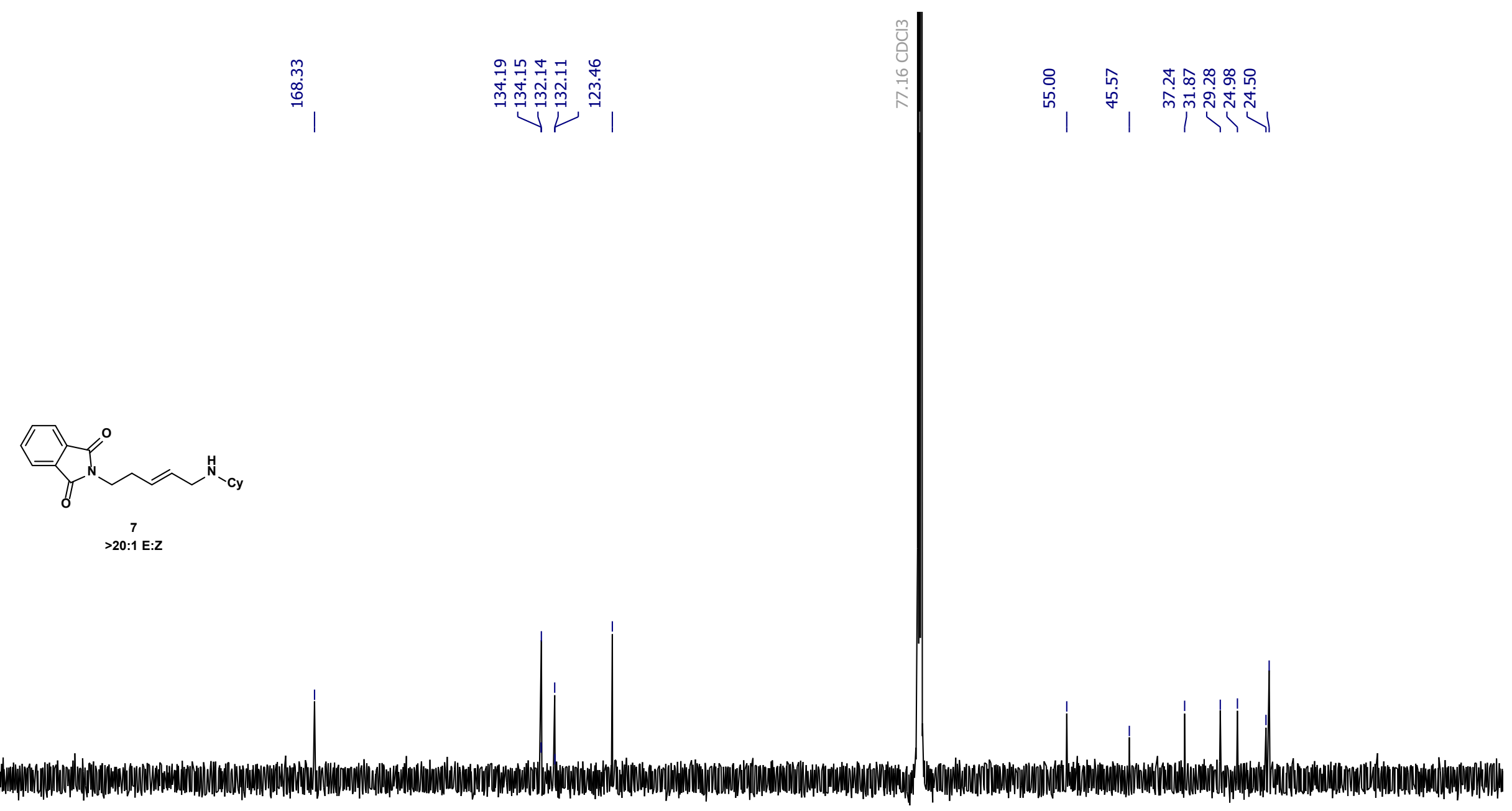

$\begin{array}{r}210 \\ 200\end{array} 190,180,170,160,150,140,130,120,110,100,90,80,70,60,50,40,30,20,10,0,-1$ f1 (ppm) 
${ }^{1} \mathrm{H}$ NMR of $(E)-4$-cyclohexylamino-2-butenyl benzoate (8)

$\mathrm{CDCl}_{3}, 25^{\circ} \mathrm{C}$

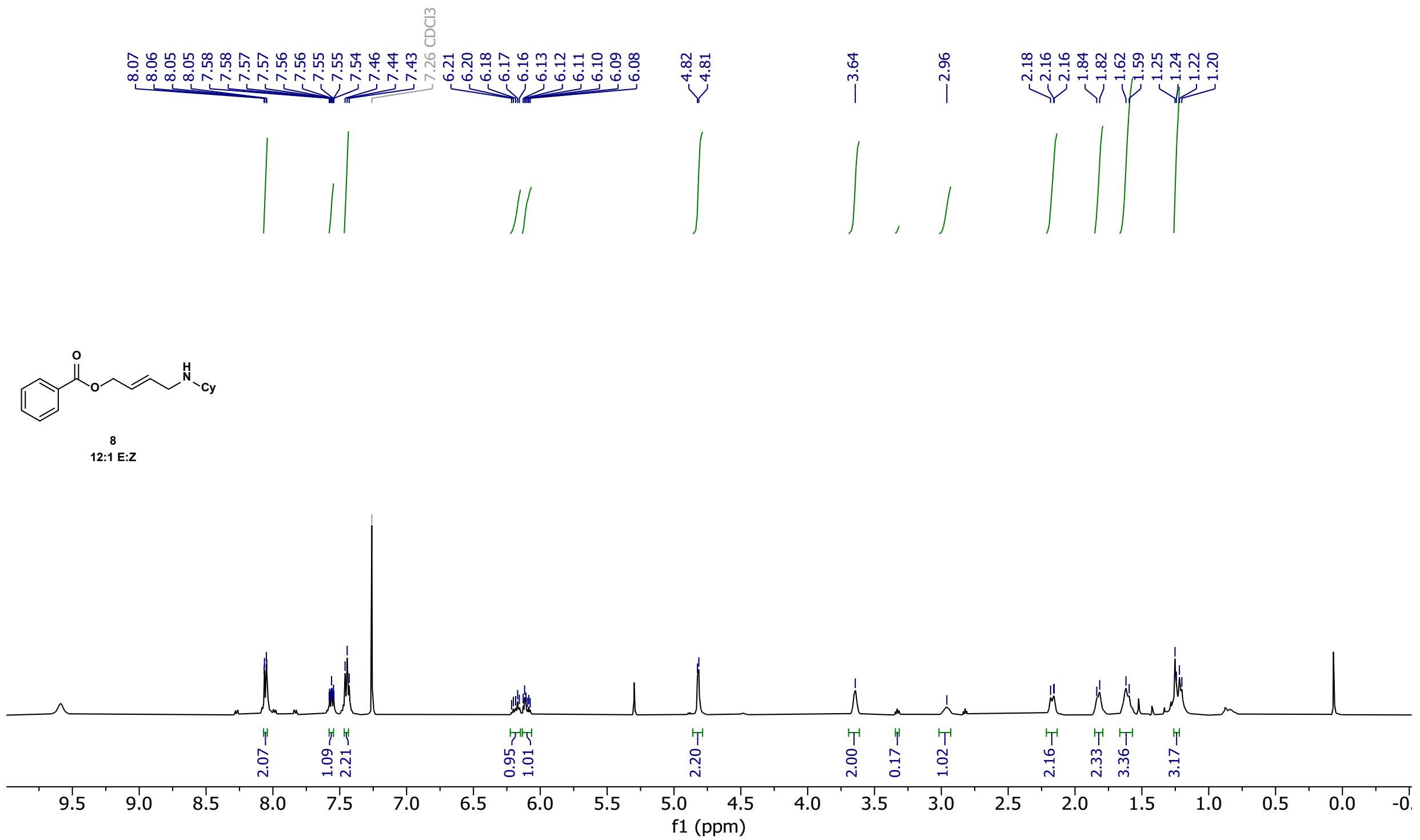


${ }^{13} \mathrm{C}$ NMR of (E)-4-cyclohexylamino-2-butenyl benzoate (8)

$\mathrm{CDCl}_{3}, 25^{\circ} \mathrm{C}$

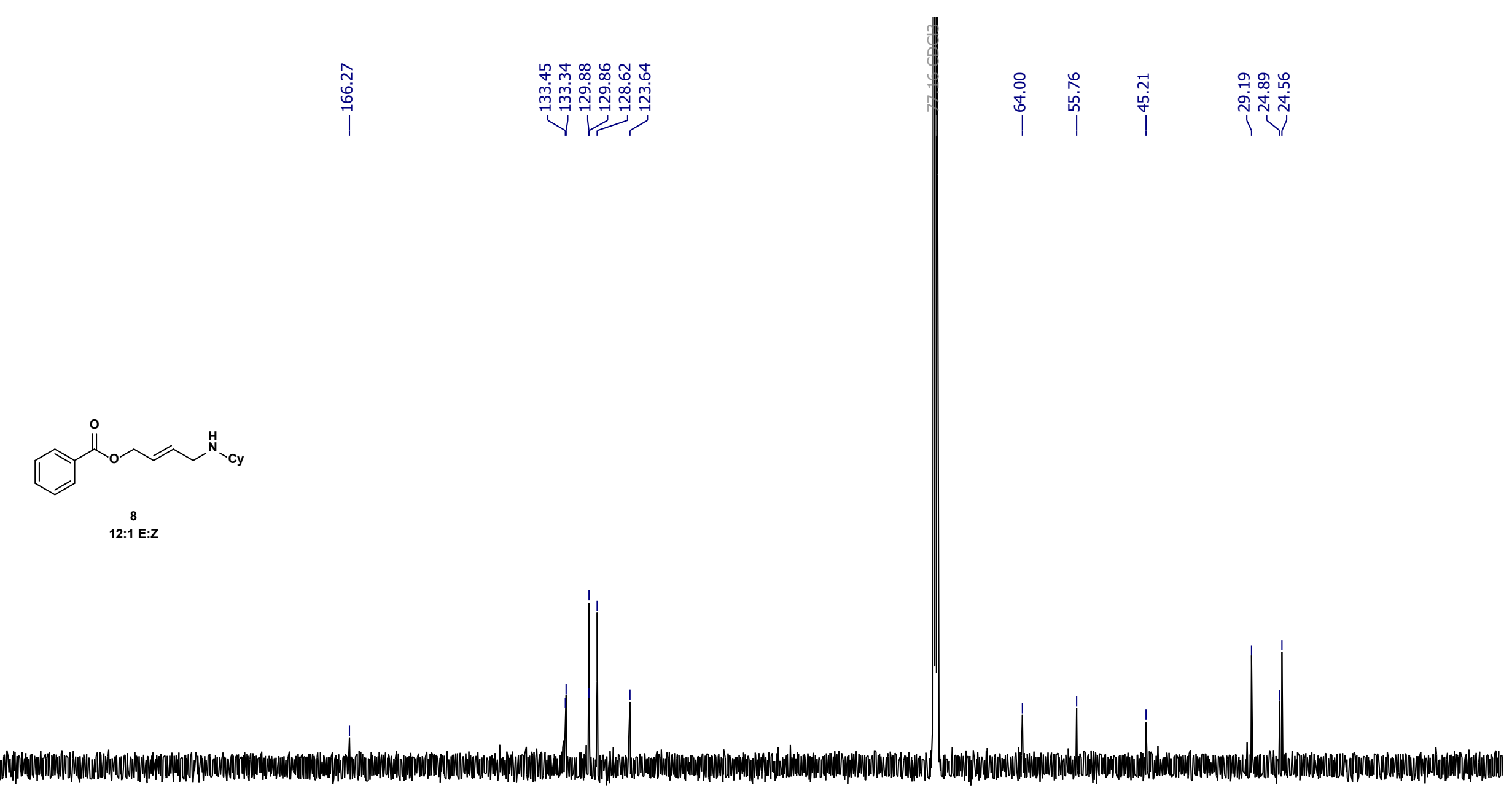

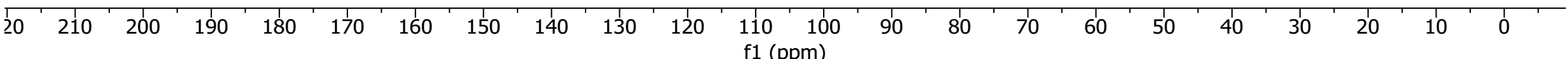


${ }^{1} \mathrm{H}$ NMR of $(E)-\mathrm{N}$-(2-dodecenyl)cyclohexanamine (9)

$\mathrm{CDCl}_{3}, 25^{\circ} \mathrm{C}$

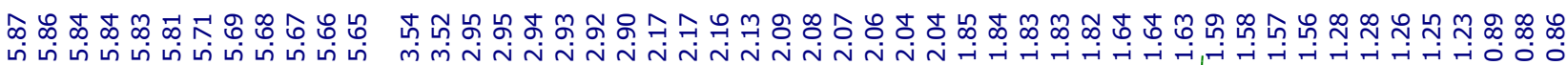

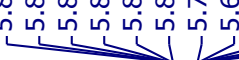
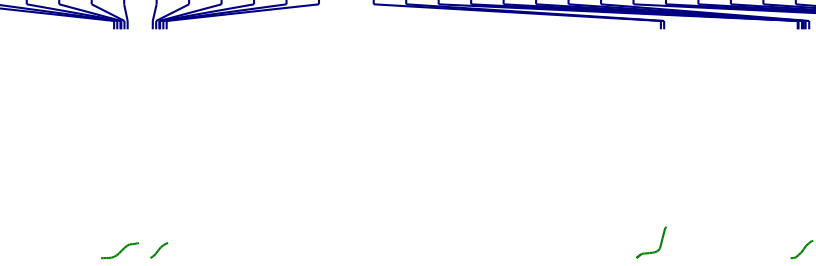

J / /
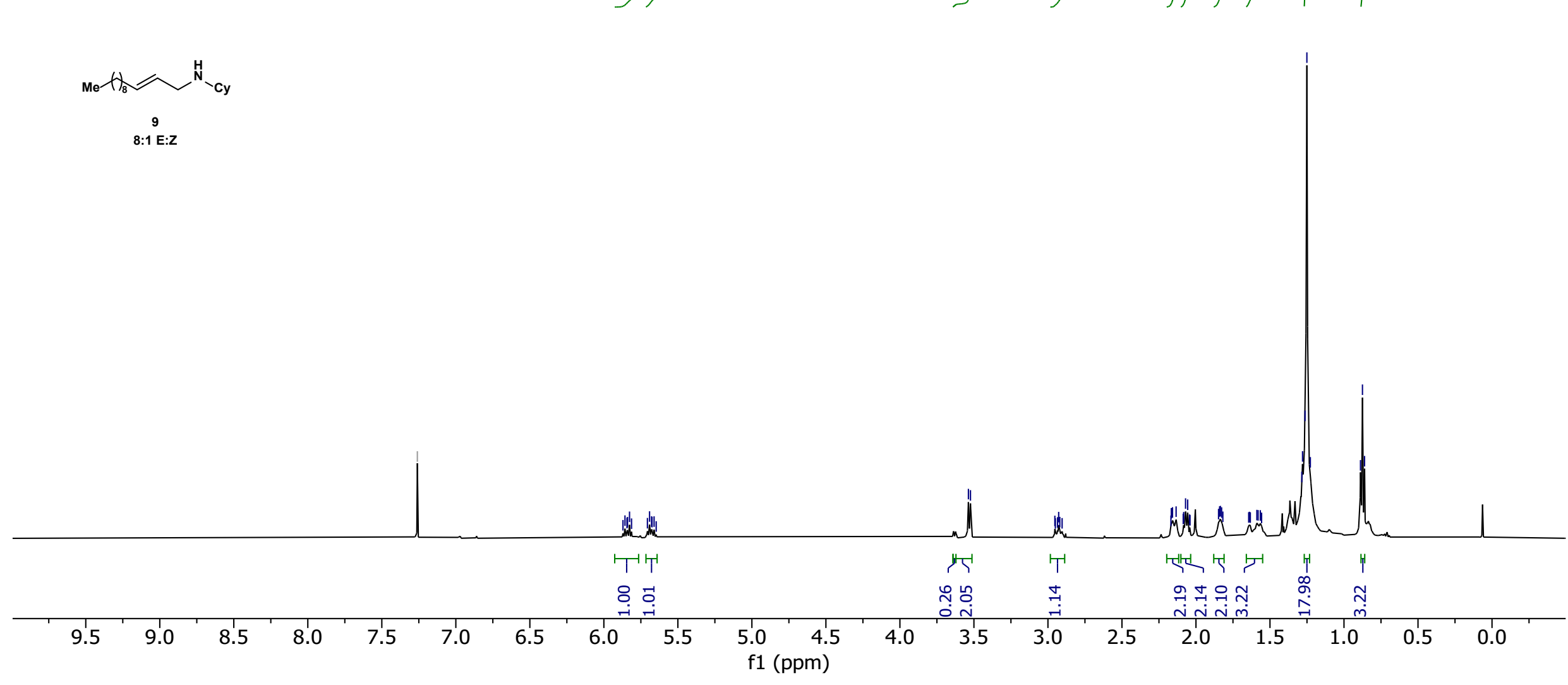
${ }^{13} \mathrm{C}$ NMR of $(E)-\mathrm{N}-(2-$ dodecenyl)cyclohexanamine (9)

$\mathrm{CDCl}_{3}, 25^{\circ} \mathrm{C}$

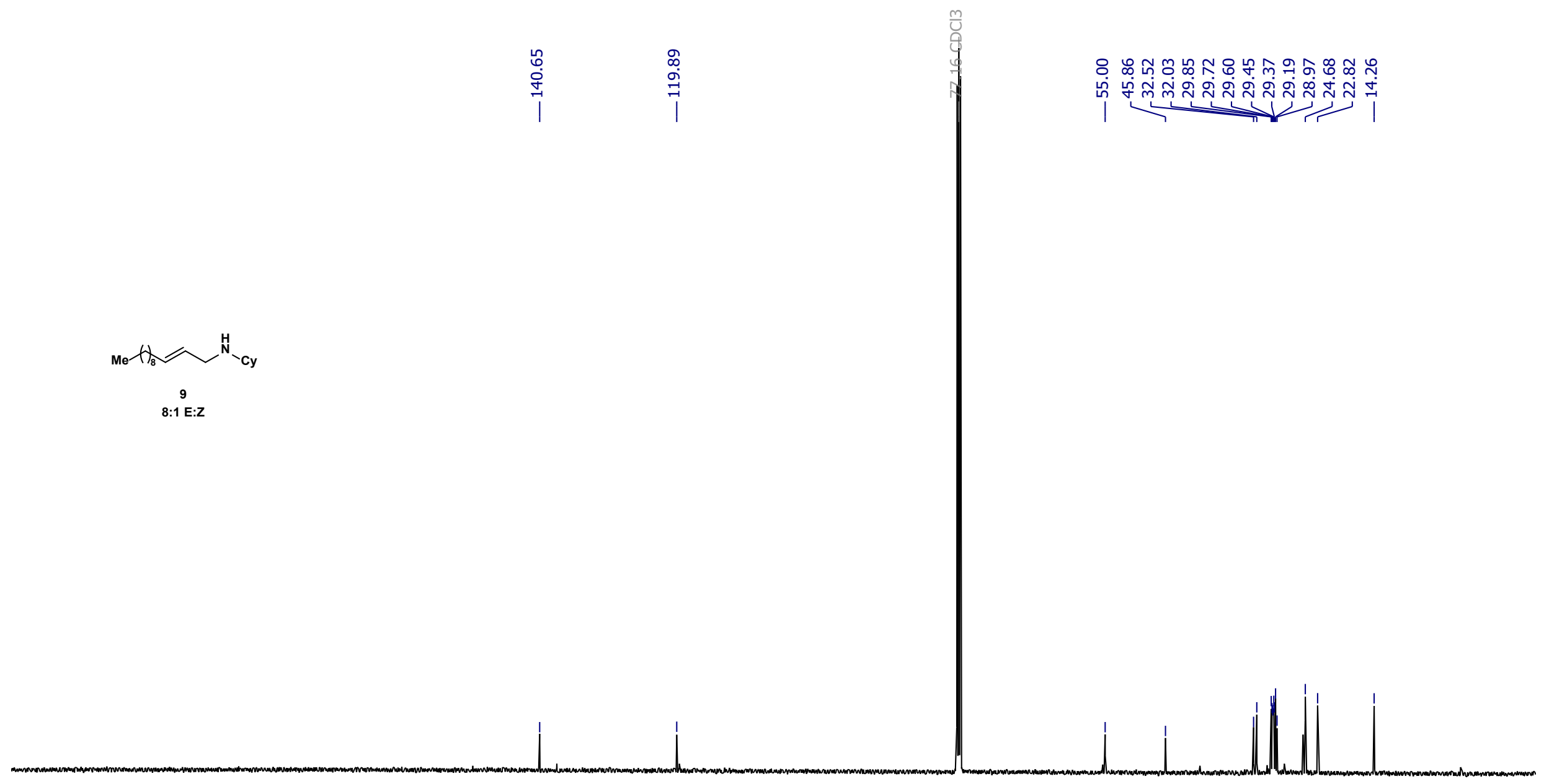

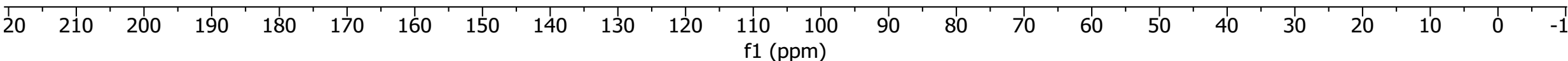




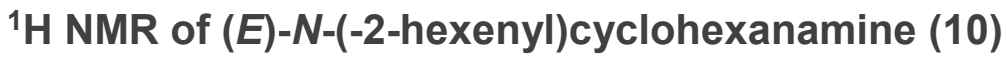

$\mathrm{CDCl}_{3}, 25^{\circ} \mathrm{C}$

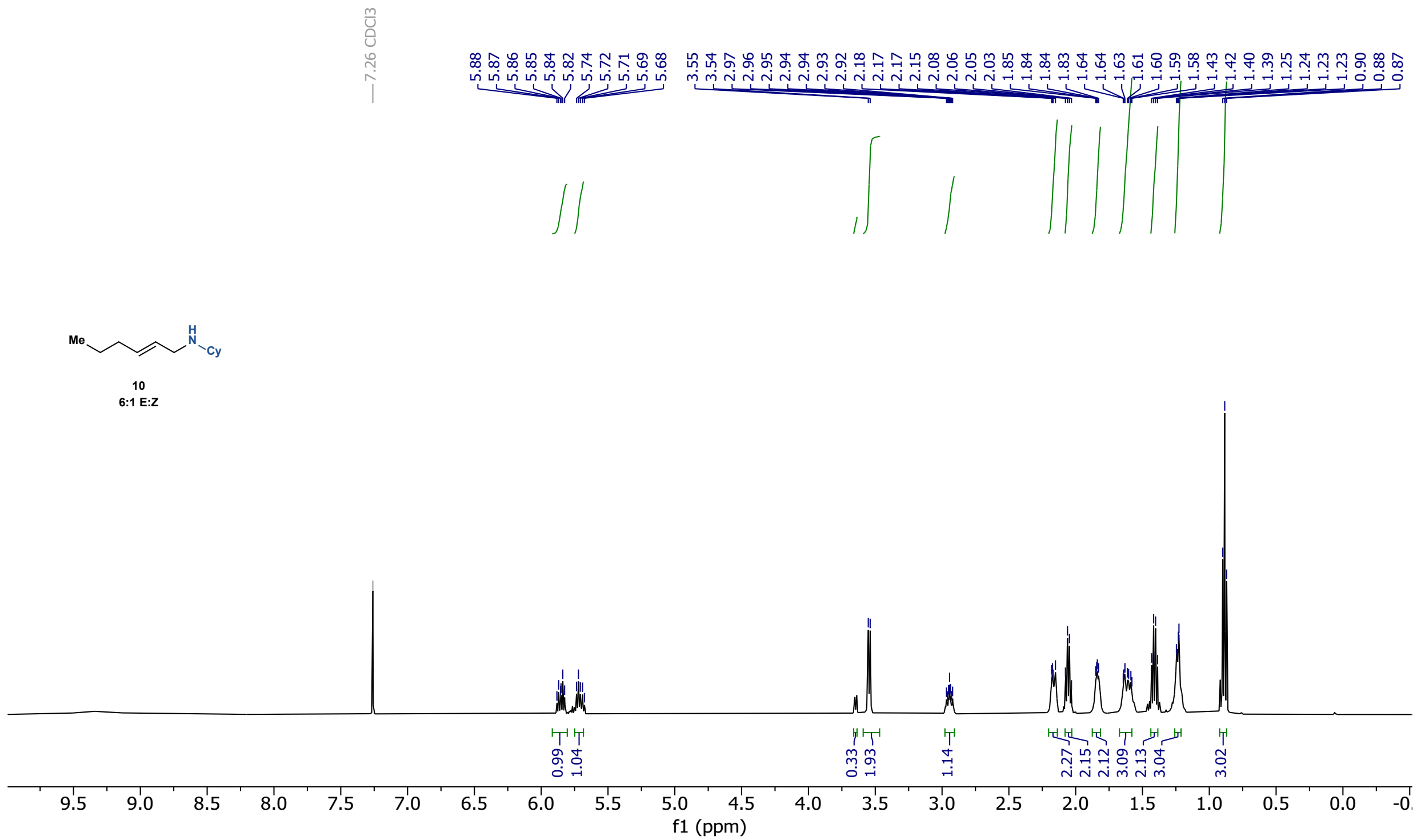




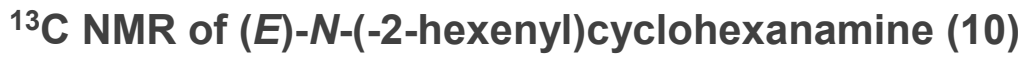

$\mathrm{CDCl}_{3}, 25{ }^{\circ} \mathrm{C}$

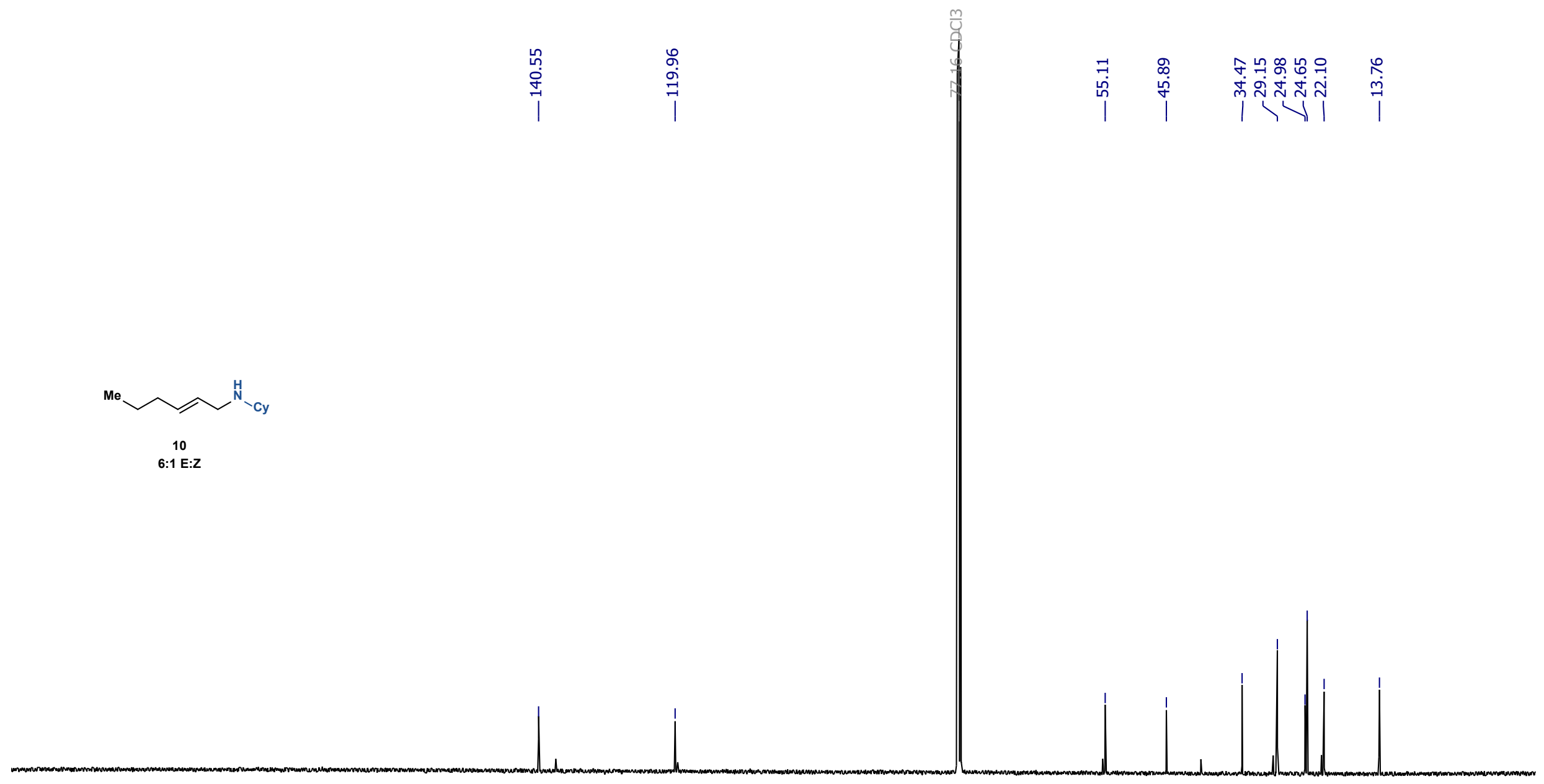

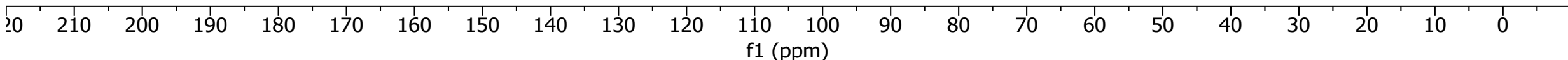


${ }^{1} \mathrm{H}$ NMR of $(E)-\mathrm{N}$-(4-methyl-2-pentenyl)cyclohexanamine (11)

$\mathrm{CDCl}_{3}, 25^{\circ} \mathrm{C}$

药

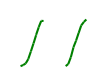

นُ

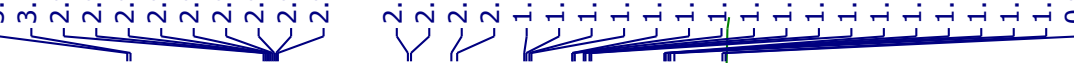<smiles>CNC/C=C/C(=[W])C(C)C</smiles>

11
$16: 1 E: z$

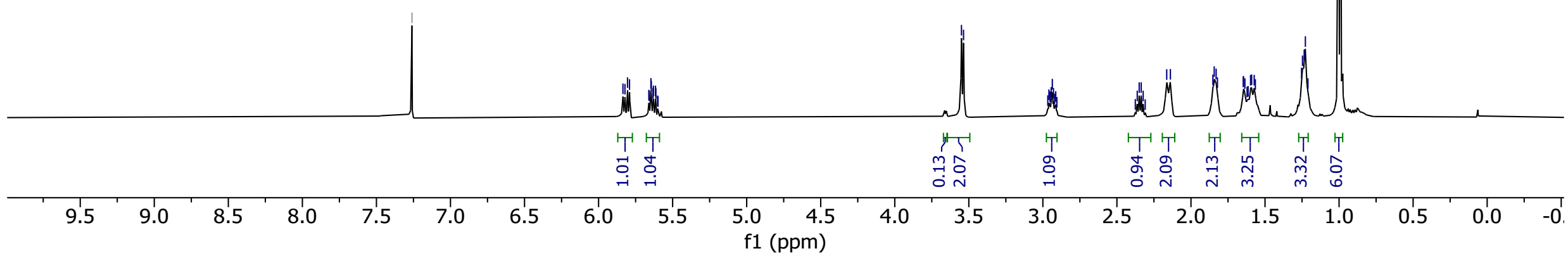


${ }^{13} \mathrm{C}$ NMR of (E)-N-(4-methyl-2-pentenyl)cyclohexanamine (11)

$\mathrm{CDCl}_{3}, 25^{\circ} \mathrm{C}$

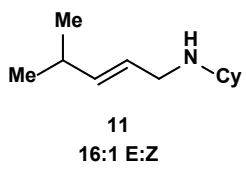


${ }^{1} \mathrm{H}$ NMR of $(E)-\mathrm{N}$-(5-(tert-butyldiphenylsilyl)oxy-2-pentenyl)cyclohexanamine (12)

$\mathrm{CDCl}_{3}, 25{ }^{\circ} \mathrm{C}$

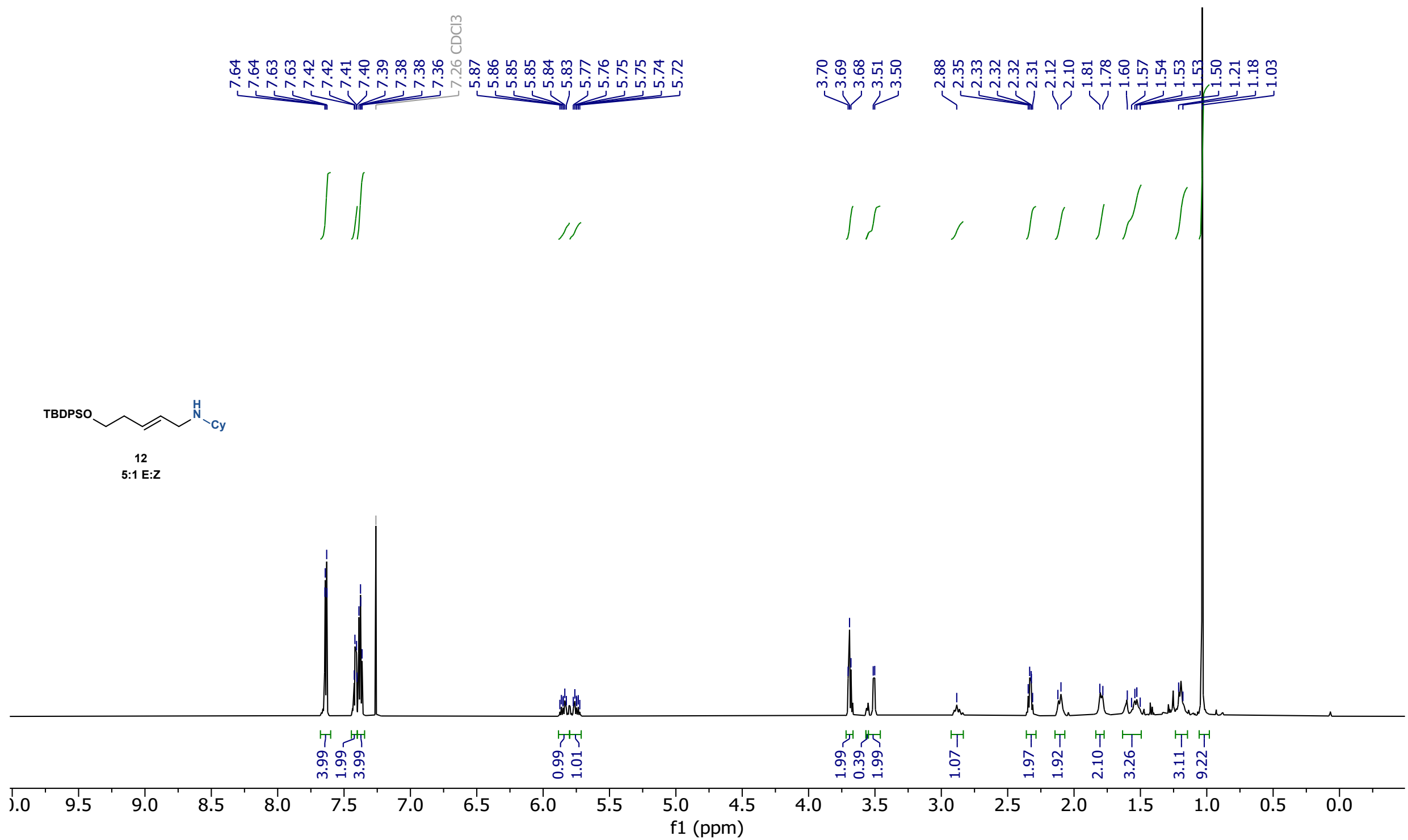


${ }^{13} \mathrm{C}$ NMR of (E)-N-(5-(tert-butyldiphenylsilyl)oxy-2-pentenyl)cyclohexanamine (12)

$\mathrm{CDCl}_{3}, 25^{\circ} \mathrm{C}$

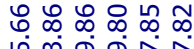

m.

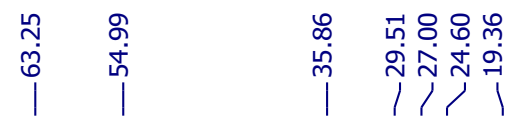

TBDPSO N

12

$5: 1 \mathrm{E} \cdot \mathrm{Z}$

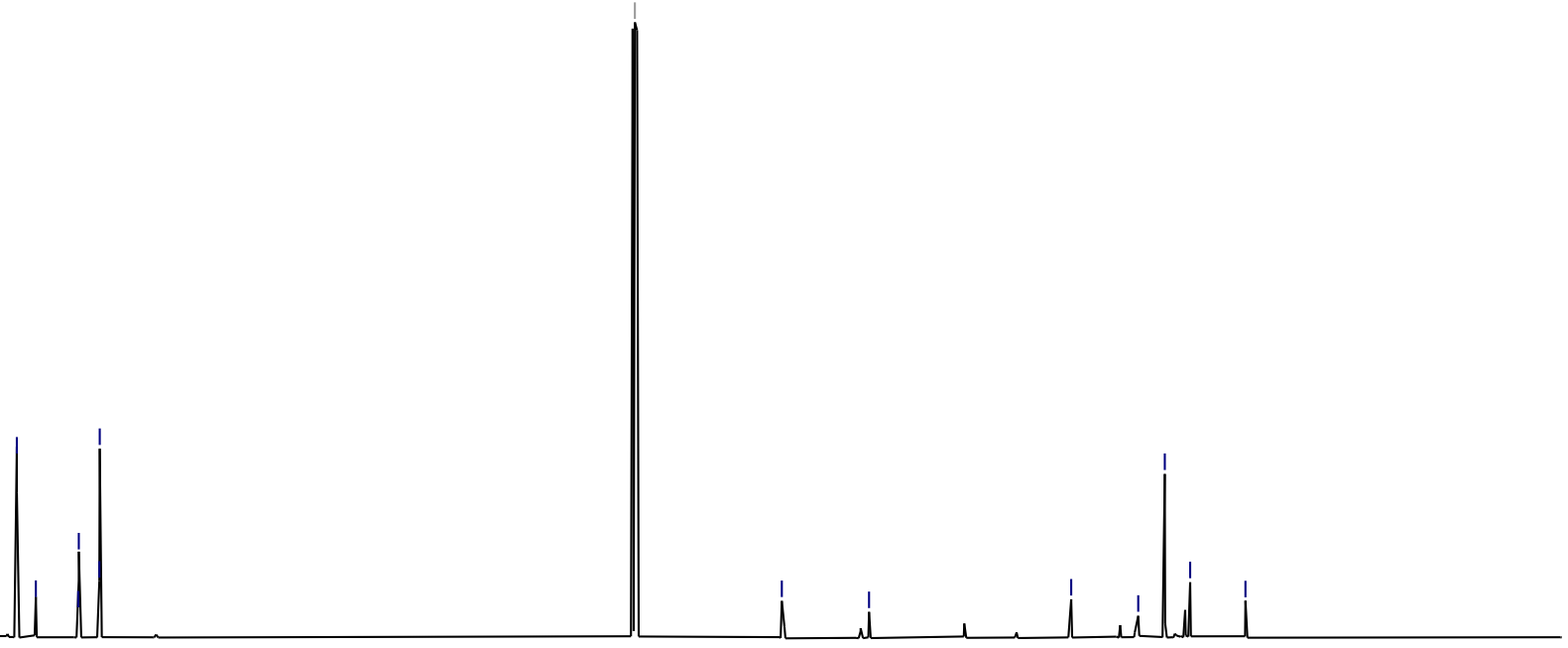

$20 \quad 210$

$210 \quad 200$

$190 \quad 180$

$170 \quad 160$

$150 \quad 140$

130

120110

f1 (ppm) 
${ }^{1} \mathrm{H}$ NMR of (E)-N-(4-bromo-2-butenyl)cyclohexanaminium trifluoroacetate (13)

$\mathrm{CDCl}_{3}, 25^{\circ} \mathrm{C}$

$\stackrel{\substack{+\infty \\ \text { aj }}}{\mid}$

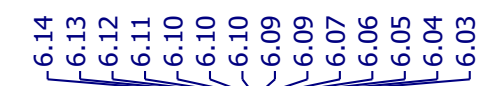

$\int$

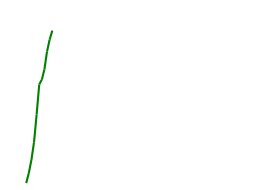

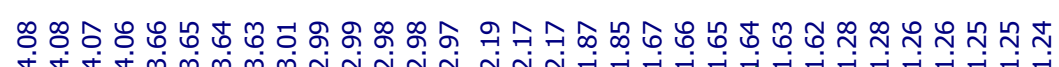
$\underbrace{4}$

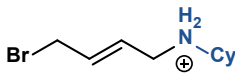

$\mathrm{CF}_{3} \mathrm{COO} \odot$

13
$10: 1 \mathrm{E}: Z$
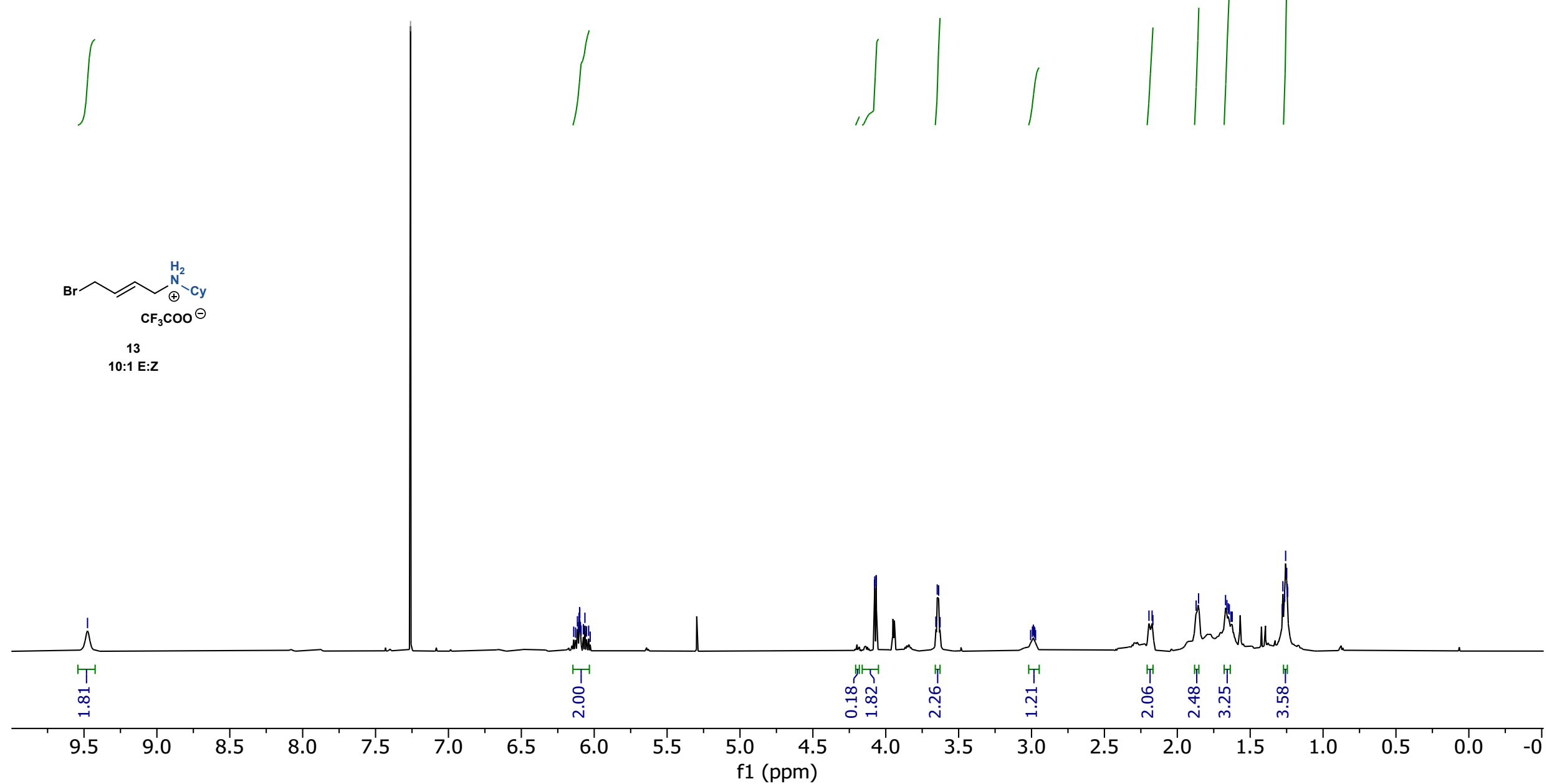
${ }^{13} \mathrm{C}$ NMR of $(E)-N$-(4-bromo-2-butenyl)cyclohexanaminium trifluoroacetate (13)

$\mathrm{CDCl}_{3}, 25^{\circ} \mathrm{C}$

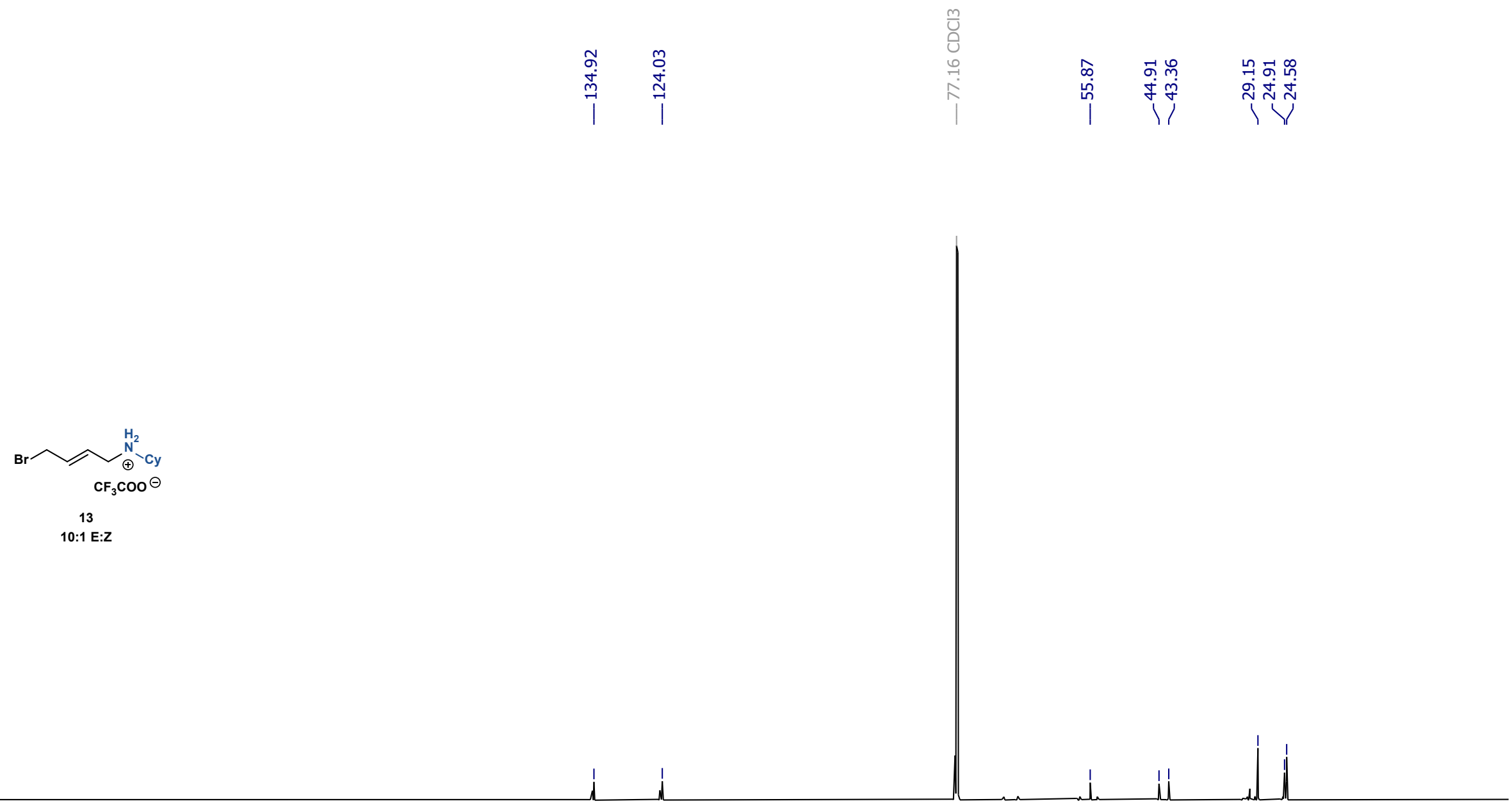

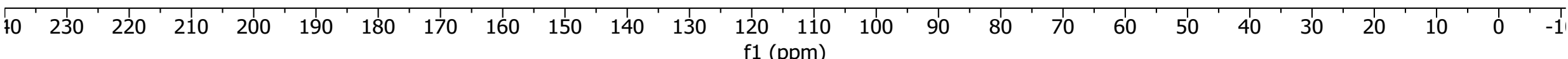


${ }^{19} \mathrm{~F}$ NMR of (E)-N-(4-bromo-2-butenyl)cyclohexanaminium trifluoroacetate (13)

$\mathrm{CDCl}_{3}, 25^{\circ} \mathrm{C}$

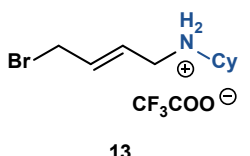

$10: 1 \mathrm{E}: \mathrm{Z}$

10:1 E:2

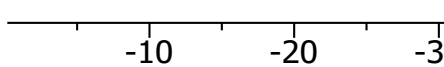

$-10 \quad-20 \quad-30 \quad-40$ f1 (ppm) 
${ }^{1} \mathrm{H}$ NMR of (E)-6-(cyclohexylamino)-4-hexen-1-ol (14)

$\mathrm{CDCl}_{3}, 25^{\circ} \mathrm{C}$

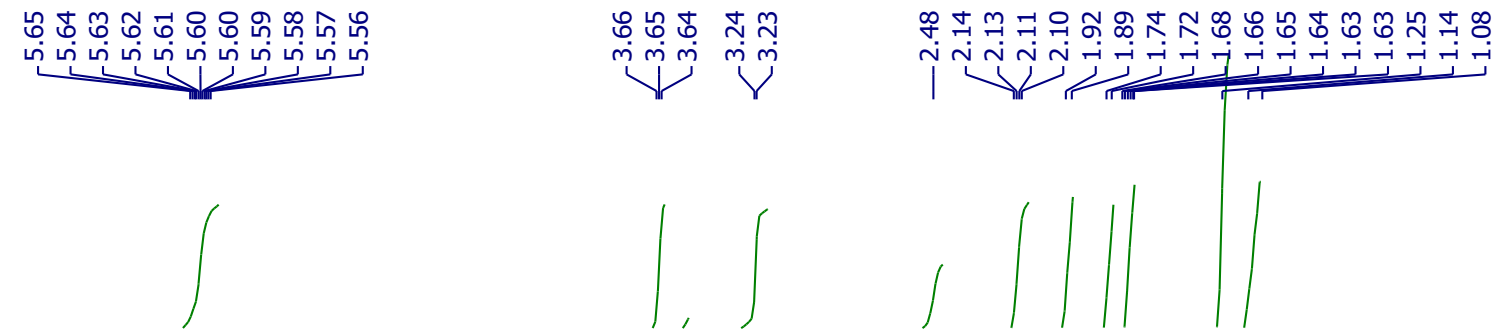

Ho $\sim^{-C y}$

$\stackrel{14}{12: 1 \mathrm{E}: \mathrm{z}}$

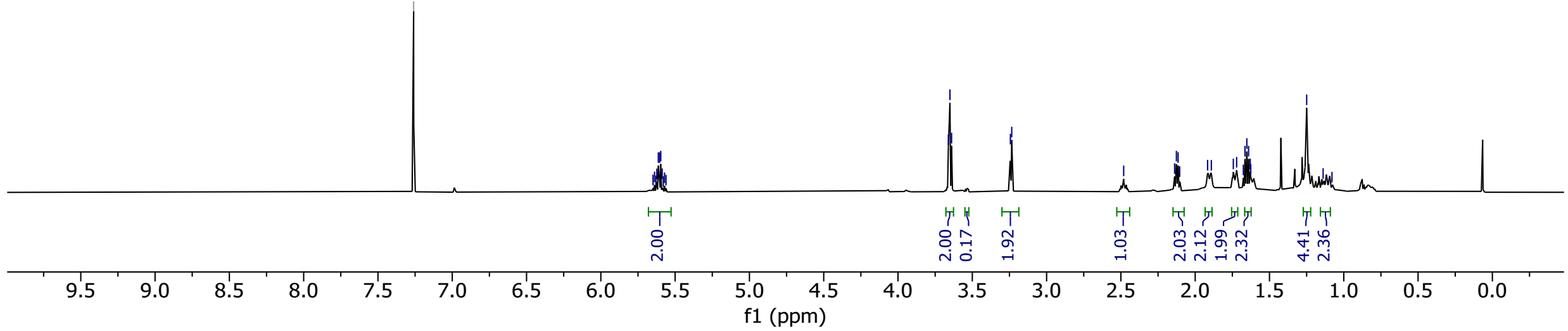


${ }^{13} \mathrm{C}$ NMR of (E)-6-(cyclohexylamino)-4-hexen-1-ol (14)

$\mathrm{CDCl}_{3}, 25^{\circ} \mathrm{C}$

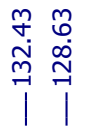

i.

HO $\sim_{\mathrm{H}^{-}}{ }^{\mathrm{Cy}}$

14

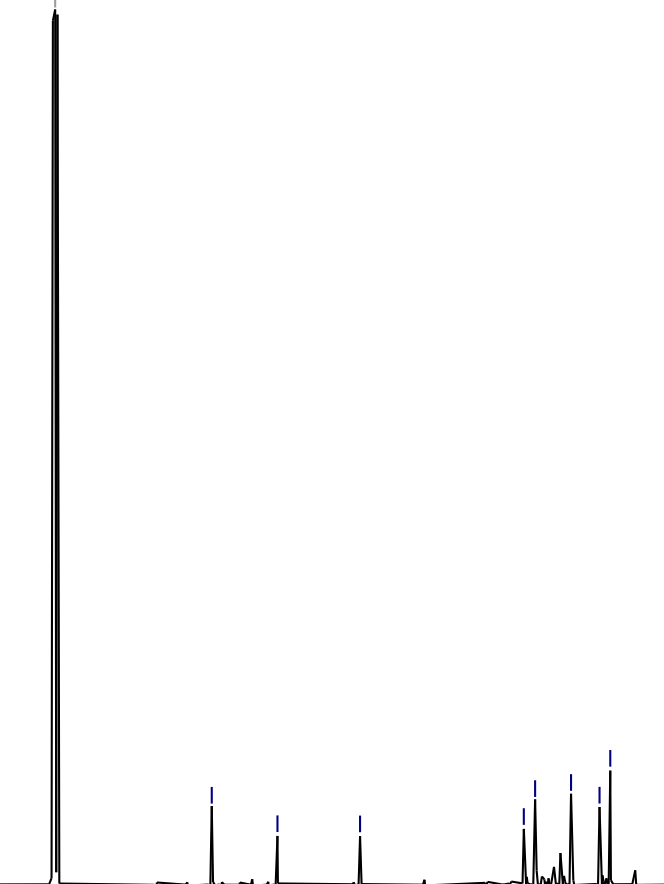

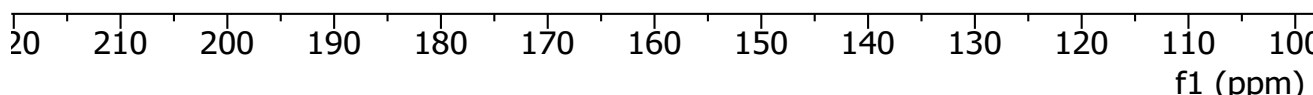


${ }^{1} \mathrm{H}$ NMR of (E)-7-cyclohexylamino-5-heptenoic acid (15)

$\mathrm{CDCl}_{3}, 25^{\circ} \mathrm{C}$

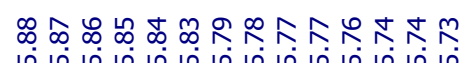

ن่

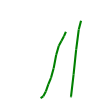

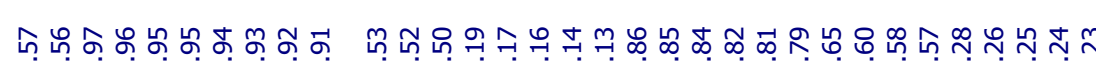

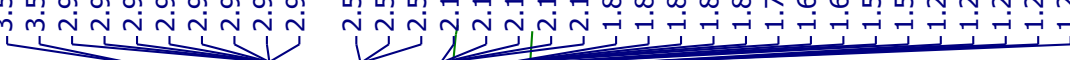

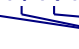

$\overbrace{15}^{\text {Hooc }} \sim_{\mathrm{H}^{-C y}}$

9:1 E:Z

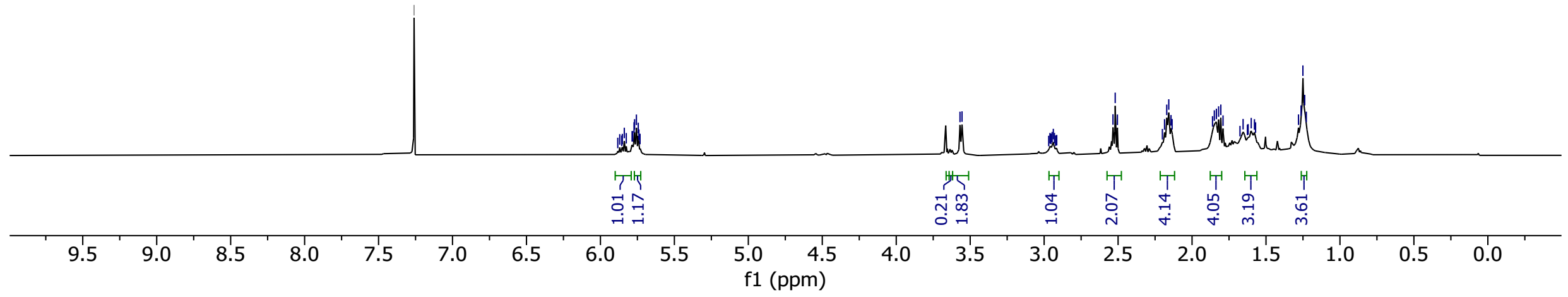


${ }^{13} \mathrm{C}$ NMR of $(E)-7-c y c l o h e x y l a m i n o-5-h e p t e n o i c$ acid (15)

$\mathrm{CDCl}_{3}, 25^{\circ} \mathrm{C}$

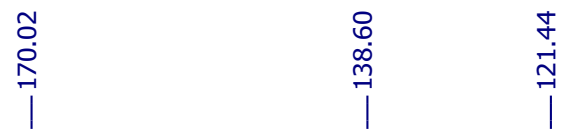

HоOс

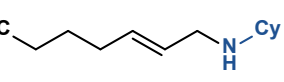

15

$9: 1 \mathrm{E} \cdot \mathrm{Z}$

崩

$\stackrel{\text { in }}{\text { in }}$

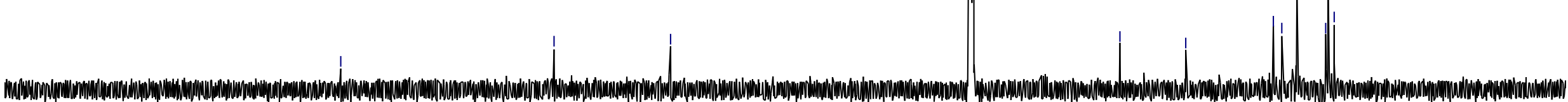

\begin{tabular}{|llllllllllllllllllllll}
210 & 200 & 190 & 180 & 170 & 160 & 150 & 140 & 130 & 120 & $\begin{array}{c}110 \\
\mathrm{f} 1(\mathrm{ppm})\end{array}$ & 90 & 80 & 70 & 60 & 50 & 40 & 30 & 20 & 10 & 0 & -1
\end{tabular}


${ }^{1} \mathrm{H}$ NMR of (E)-N-(2-octen-7-ynyl)cyclohexanamine (16)

$\mathrm{CDCl}_{3}, 25^{\circ} \mathrm{C}$

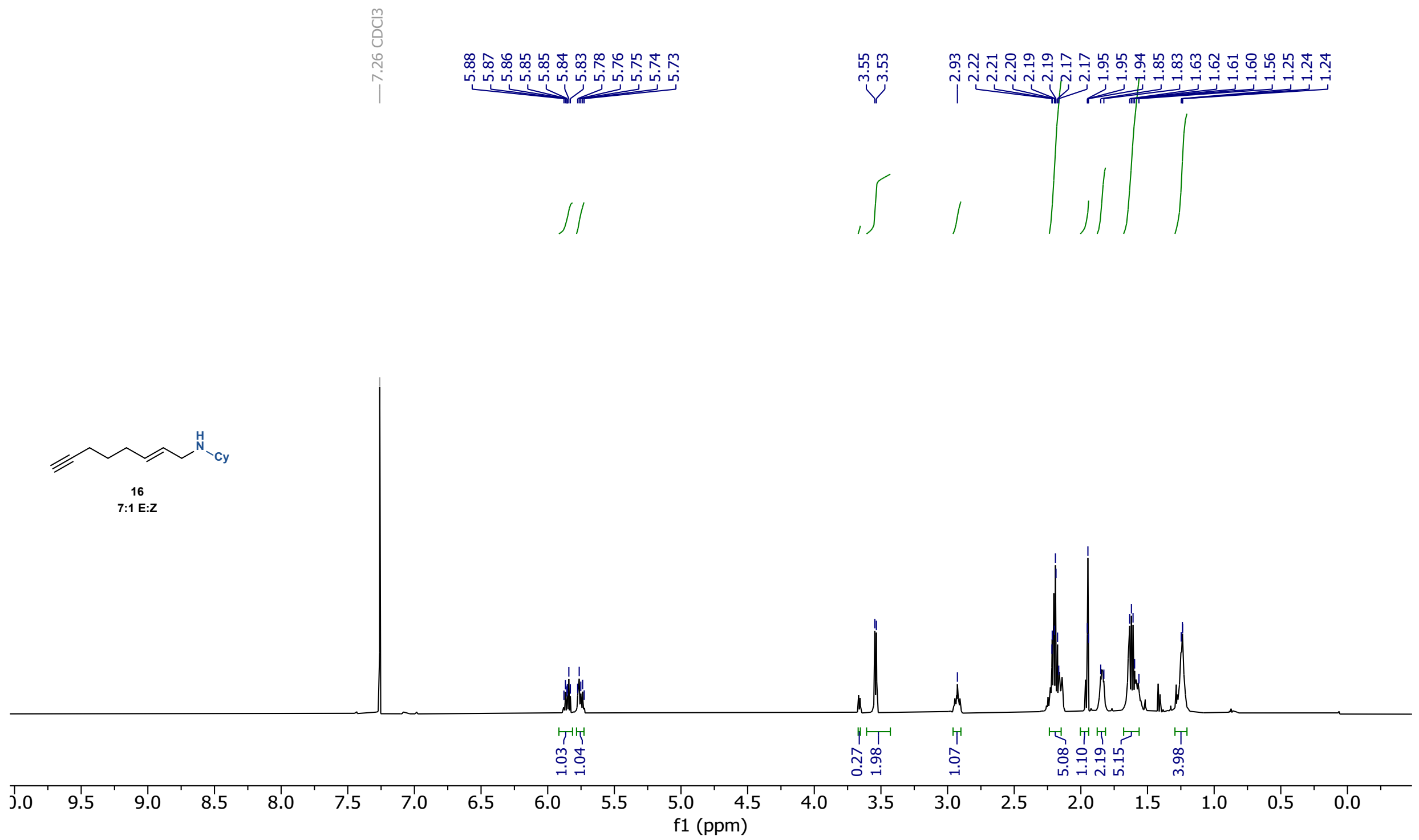




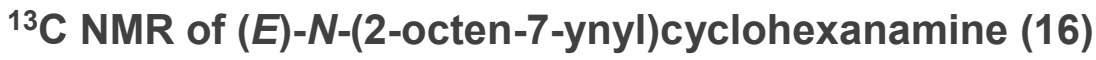

$\mathrm{CDCl}_{3}, 25{ }^{\circ} \mathrm{C}$

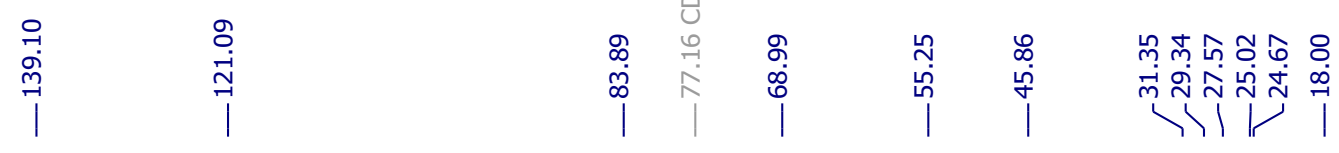
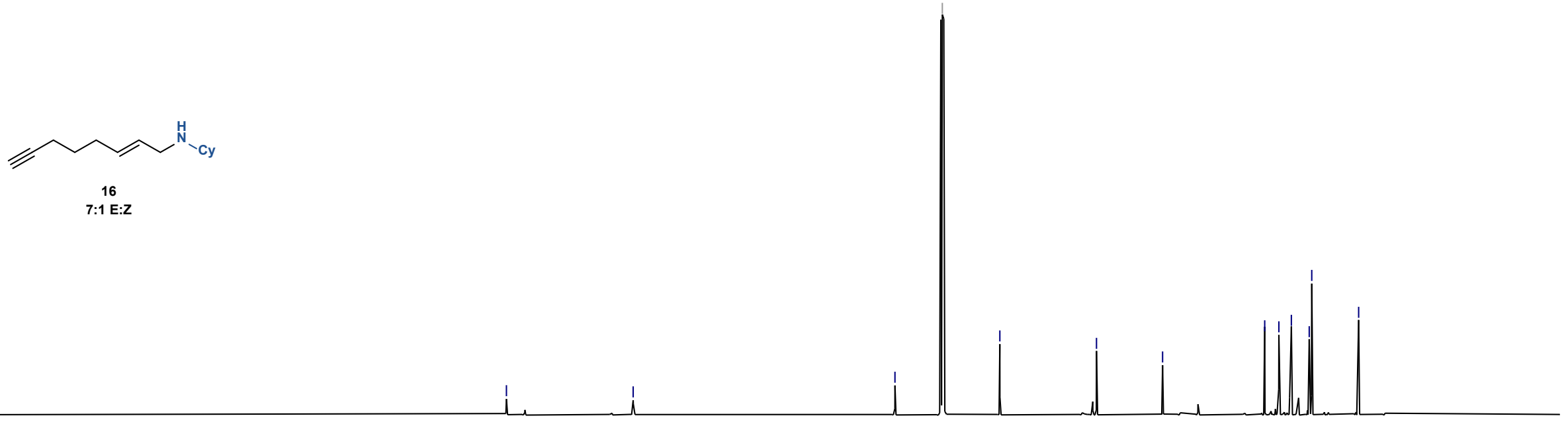

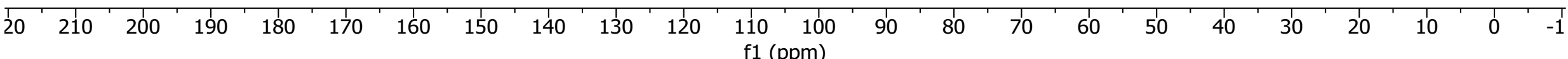


${ }^{1} \mathrm{H}$ NMR of $(E)-\mathrm{N}-(2,5$-hexadienyl)cyclohexanamine (17)

$\mathrm{CDCl}_{3}, 25^{\circ} \mathrm{C}$

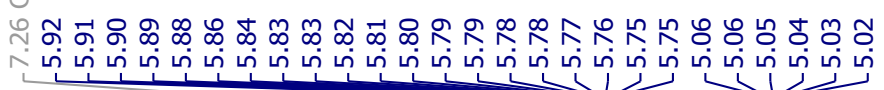
1

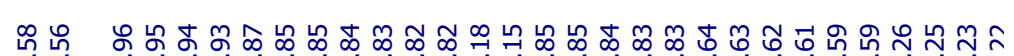

min

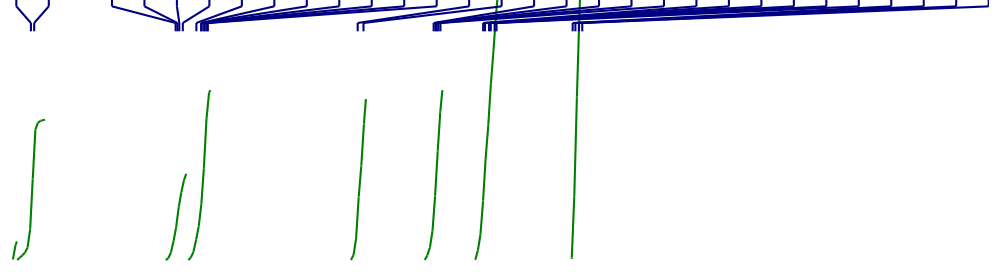

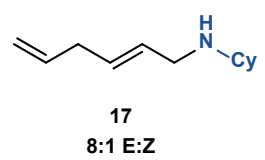

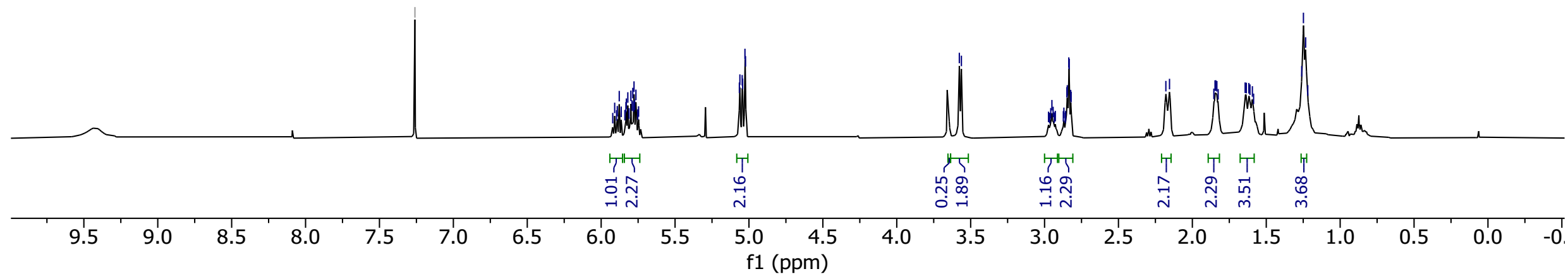


${ }^{13} \mathrm{C}$ NMR of $(E)-\mathrm{N}-(2,5$-hexadienyl)cyclohexanamine (17)

$\mathrm{CDCl}_{3}, 25^{\circ} \mathrm{C}$

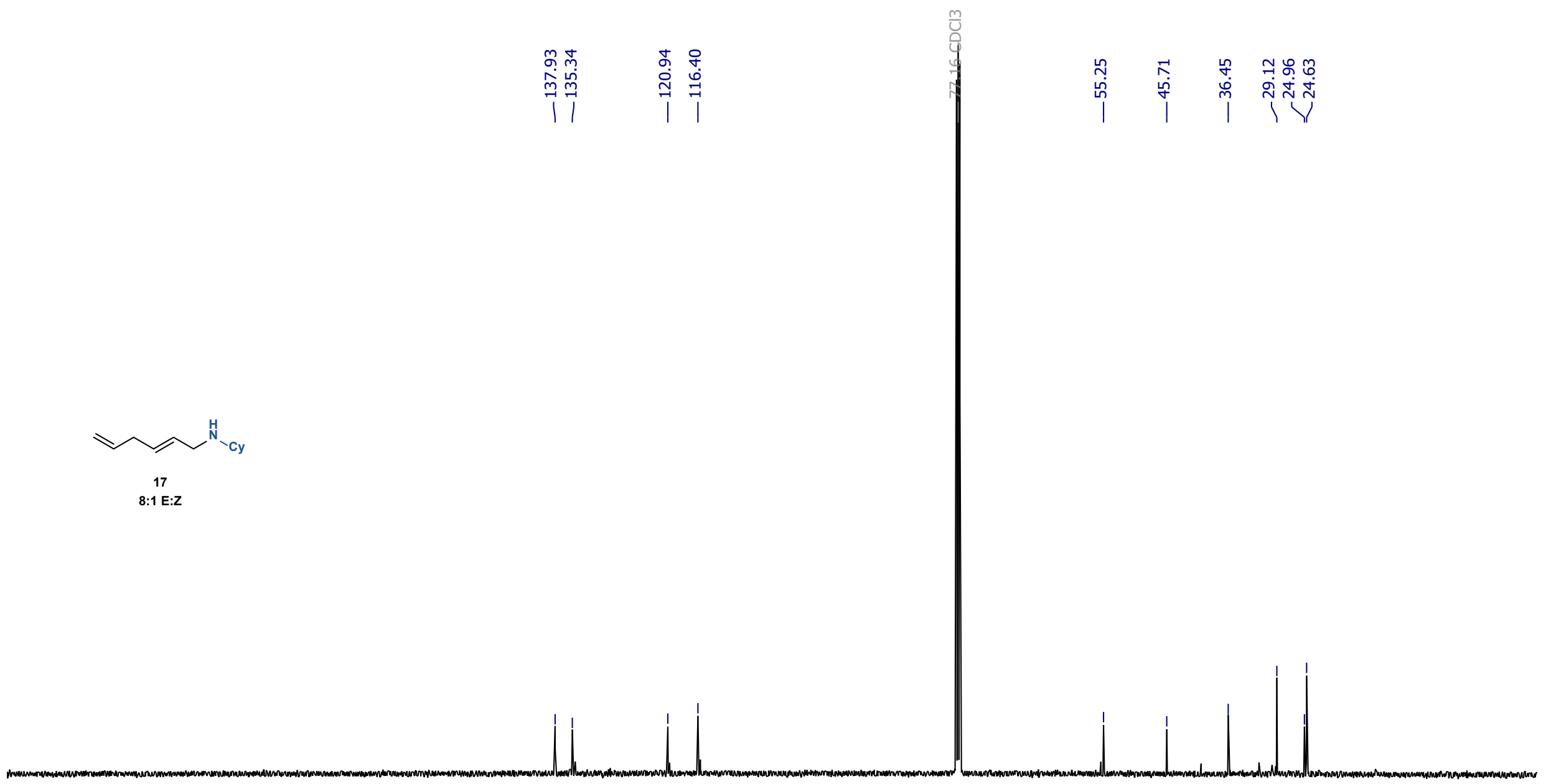

\begin{tabular}{|l|llllllllllllllllllllllllll}
\hline 20 & 210 & 200 & 190 & 180 & 170 & 160 & 150 & 140 & 130 & 120 & $\begin{array}{c}110 \\
\mathrm{f} 1(\mathrm{ppm})\end{array}$ & 100 & 90 & 80 & 70 & 60 & 50 & 40 & 30 & 20 & 10 & 0 & -1
\end{tabular} 
${ }^{1} \mathrm{H}$ NMR of $\mathrm{N}$-(2-(bicyclo[2.2.1]heptan-2-ylidene)ethyl)cyclohexanamine (18)

$\mathrm{CDCl}_{3}, 25^{\circ} \mathrm{C}$
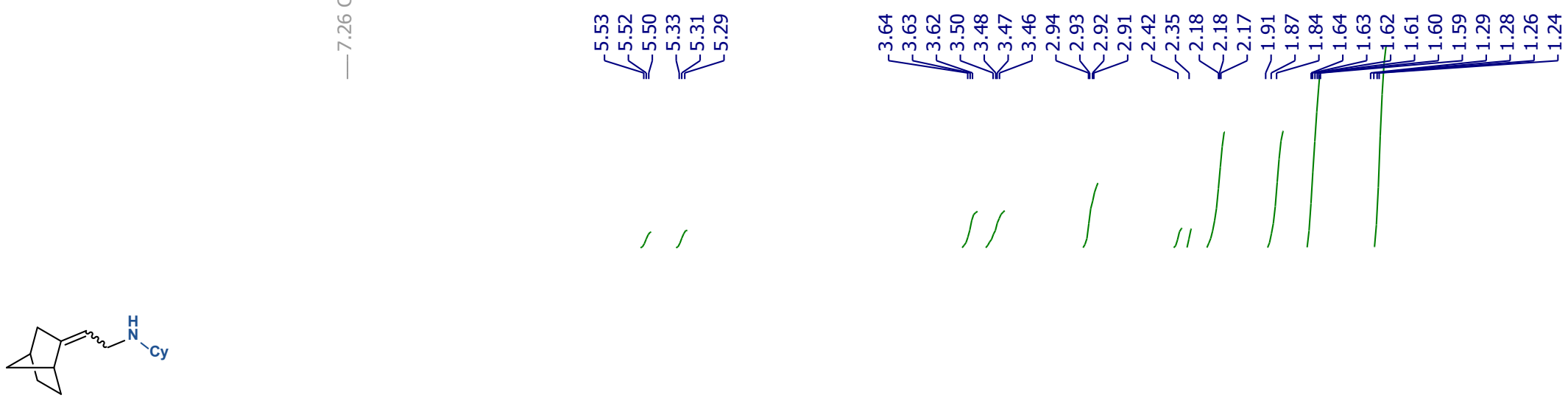

18

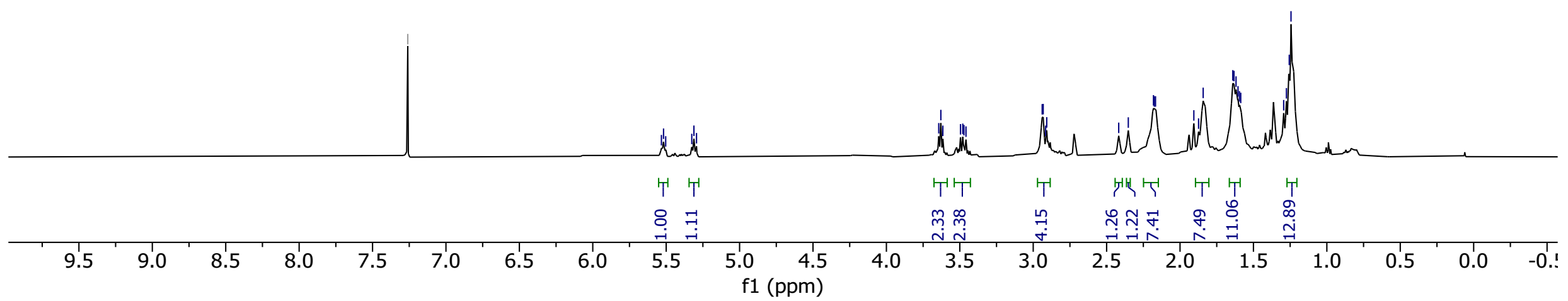


${ }^{13} \mathrm{C}$ NMR of $\mathrm{N}$-(2-(bicyclo[2.2.1]heptan-2-ylidene)ethyl)cyclohexanamine (18)

$\mathrm{CDCl}_{3}, 25^{\circ} \mathrm{C}$

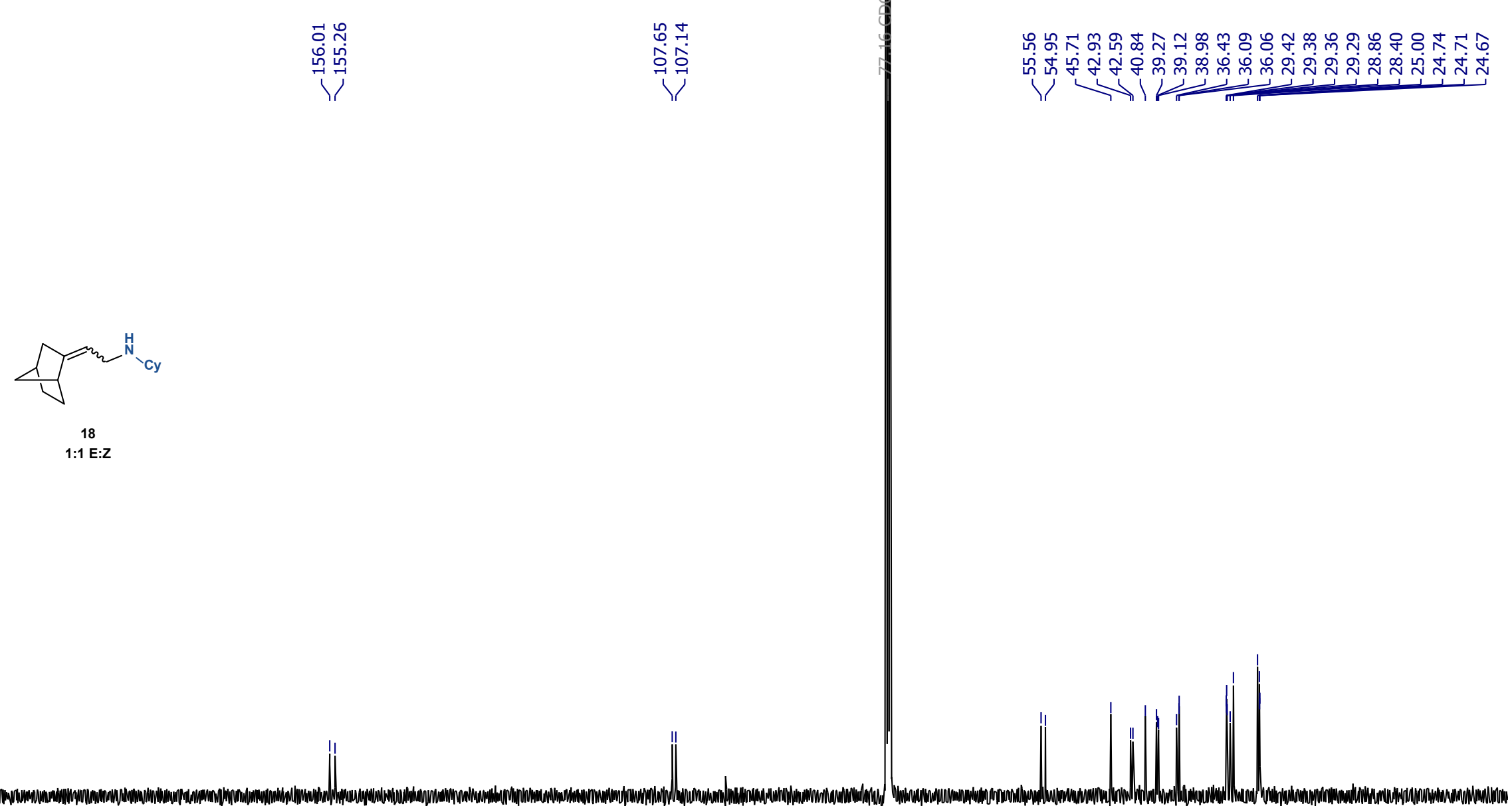

\begin{tabular}{llllllllllllllllllllllllll}
\hline & 210 & 200 & 190 & 180 & 170 & 160 & 150 & 140 & 130 & 120 & $\begin{array}{c}110 \\
\mathrm{f} 1(\mathrm{ppm})\end{array}$ & 90 & 80 & 70 & 60 & 50 & 40 & 30 & 20 & 10 & 0 & -1
\end{tabular}


${ }^{1} \mathrm{H}$ NMR of $\mathrm{N}$-(2-cyclohexylideneethyl)cyclohexanamine (19)

$\mathrm{CDCl}_{3}, 25^{\circ} \mathrm{C}$

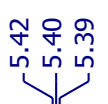

|n

$\int$

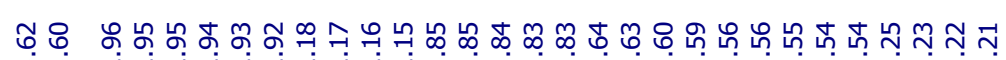

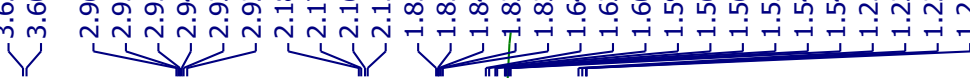
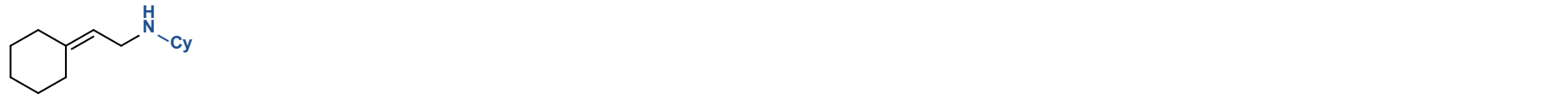
${ }^{13} \mathrm{C}$ NMR of $\mathrm{N}$-(2-cyclohexylideneethyl)cyclohexanamine (19)

$\mathrm{CDCl}_{3}, 25^{\circ} \mathrm{C}$

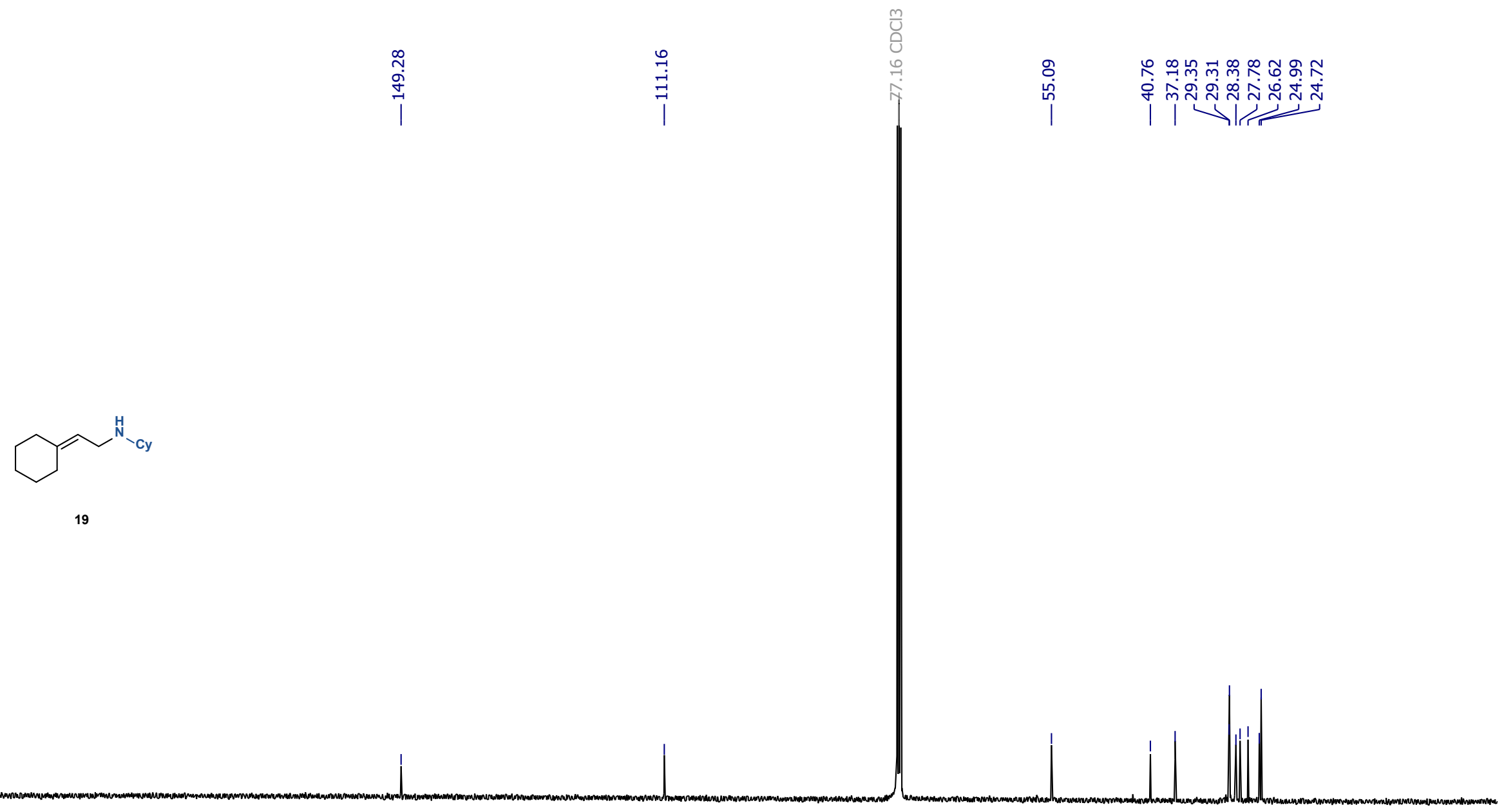

$\begin{array}{llllllllllll}210 & 200 & 190 & 180 & 170 & 160 & 150 & 140 & 130 & 120 & 110 & 100\end{array}$

f1 (ppm)

$\begin{array}{llllllllll}90 & 80 & 70 & 60 & 50 & 40 & 30 & 20 & 10 & 0\end{array}$ 
${ }^{1} \mathrm{H}$ NMR of (Z)-N-(3-phenylallyl)cyclohexanamine (20)

$\mathrm{CDCl}_{3}, 25^{\circ} \mathrm{C}$

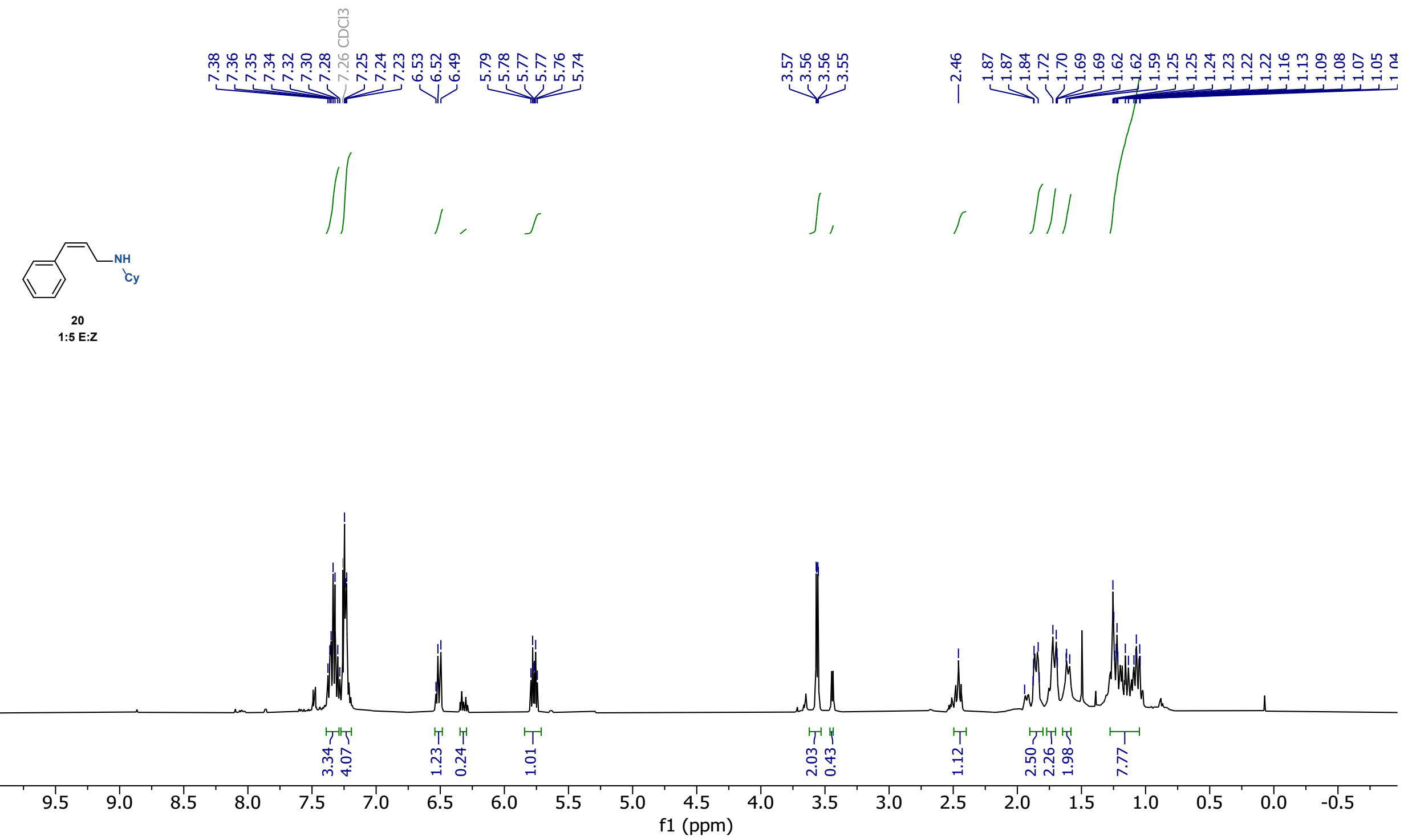


${ }^{13} \mathrm{C}$ NMR of (Z)-N-(3-phenylallyl)cyclohexanamine (20)

$\mathrm{CDCl}_{3}, 25^{\circ} \mathrm{C}$

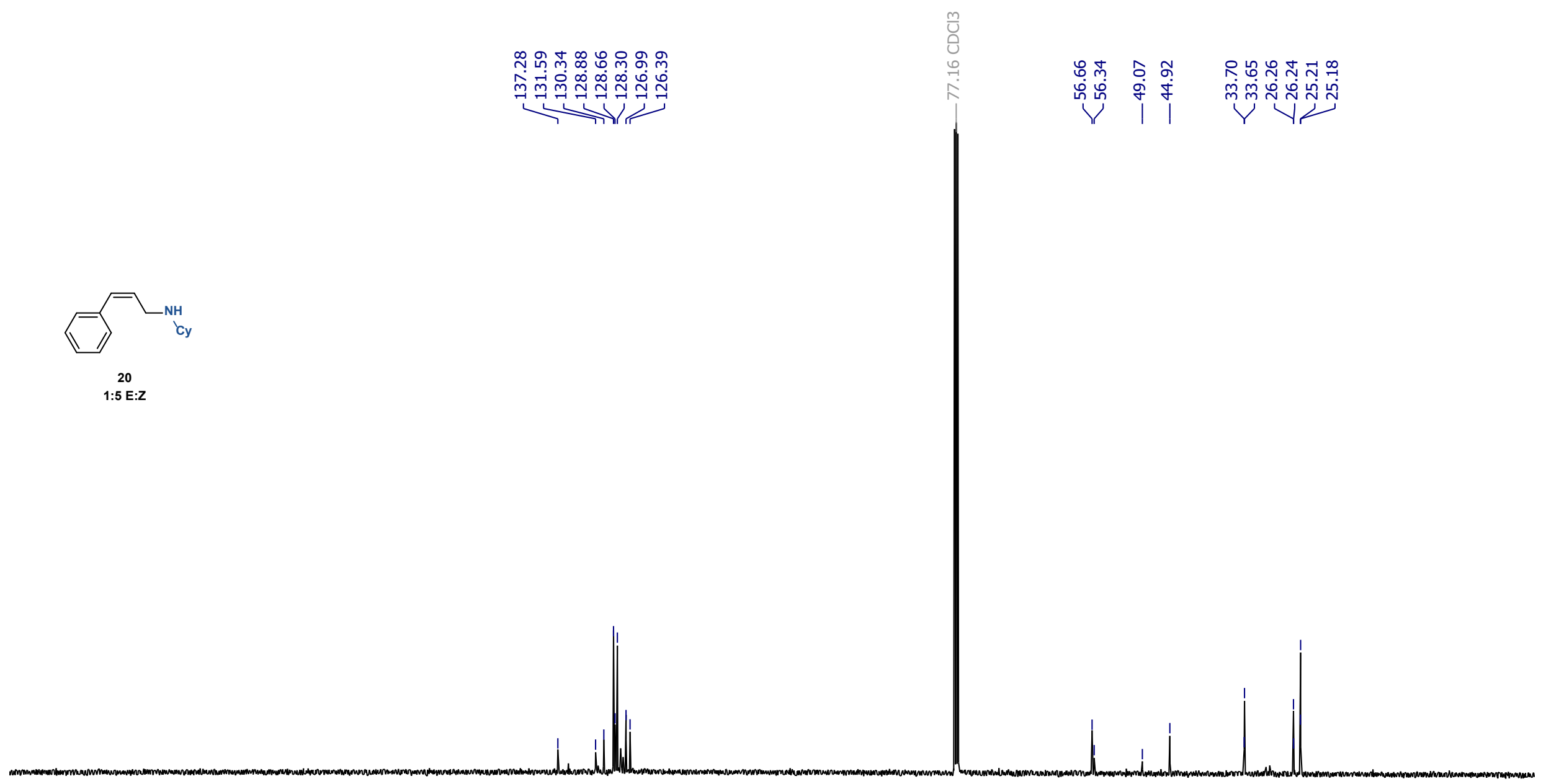

\begin{tabular}{lllllllllllllllllllllllllllll}
\hline & 210 & 200 & 190 & 180 & 170 & 160 & 150 & 140 & 130 & 120 & 110 & 100 & 90 & 80 & 70 & 60 & 50 & 40 & 30 & 20 & 10 & 0 & -1
\end{tabular} 
${ }^{1} \mathrm{H}$ NMR of $(E)-\mathrm{N}$-(5-octen-4-yl)cyclohexanamine (21)

$\mathrm{CDCl}_{3}, 25^{\circ} \mathrm{C}$

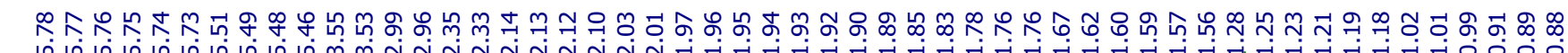

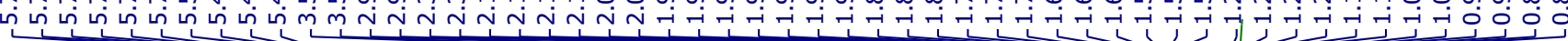
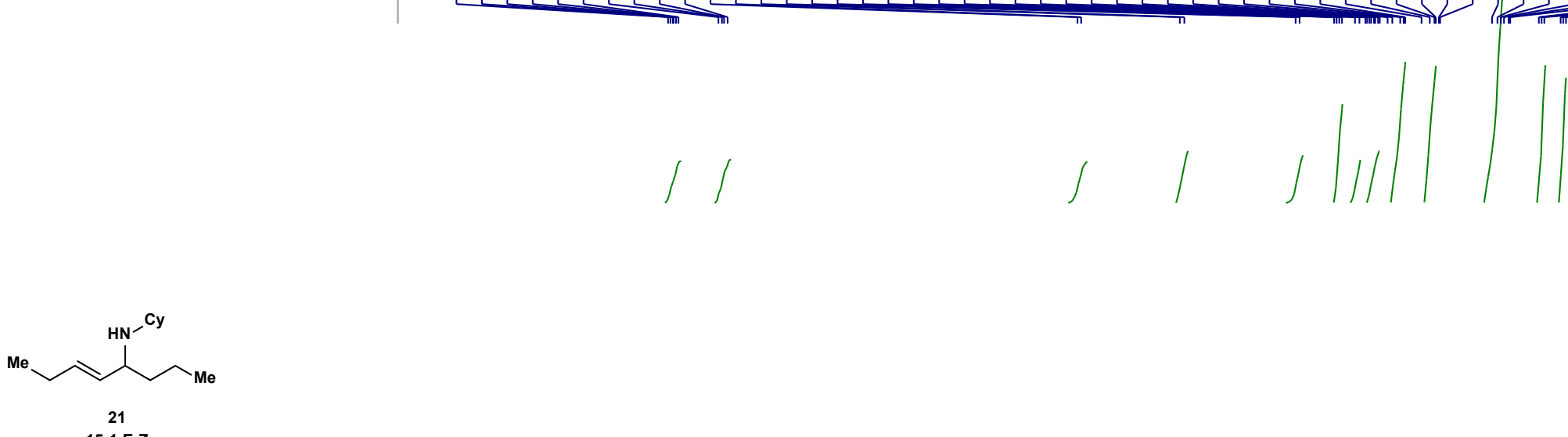

15:1 E:Z

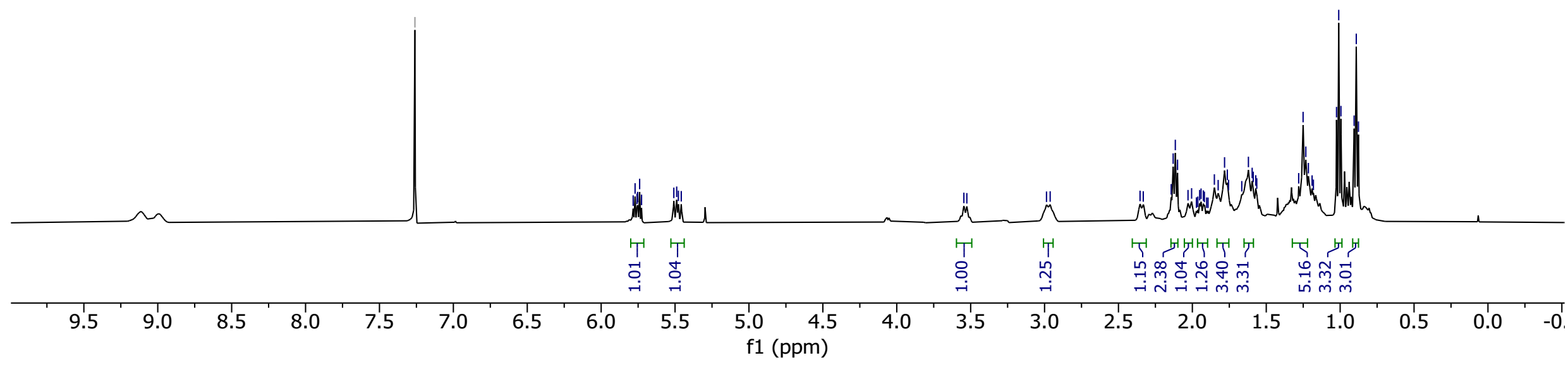




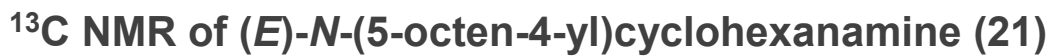

$\mathrm{CDCl}_{3}, 25^{\circ} \mathrm{C}$

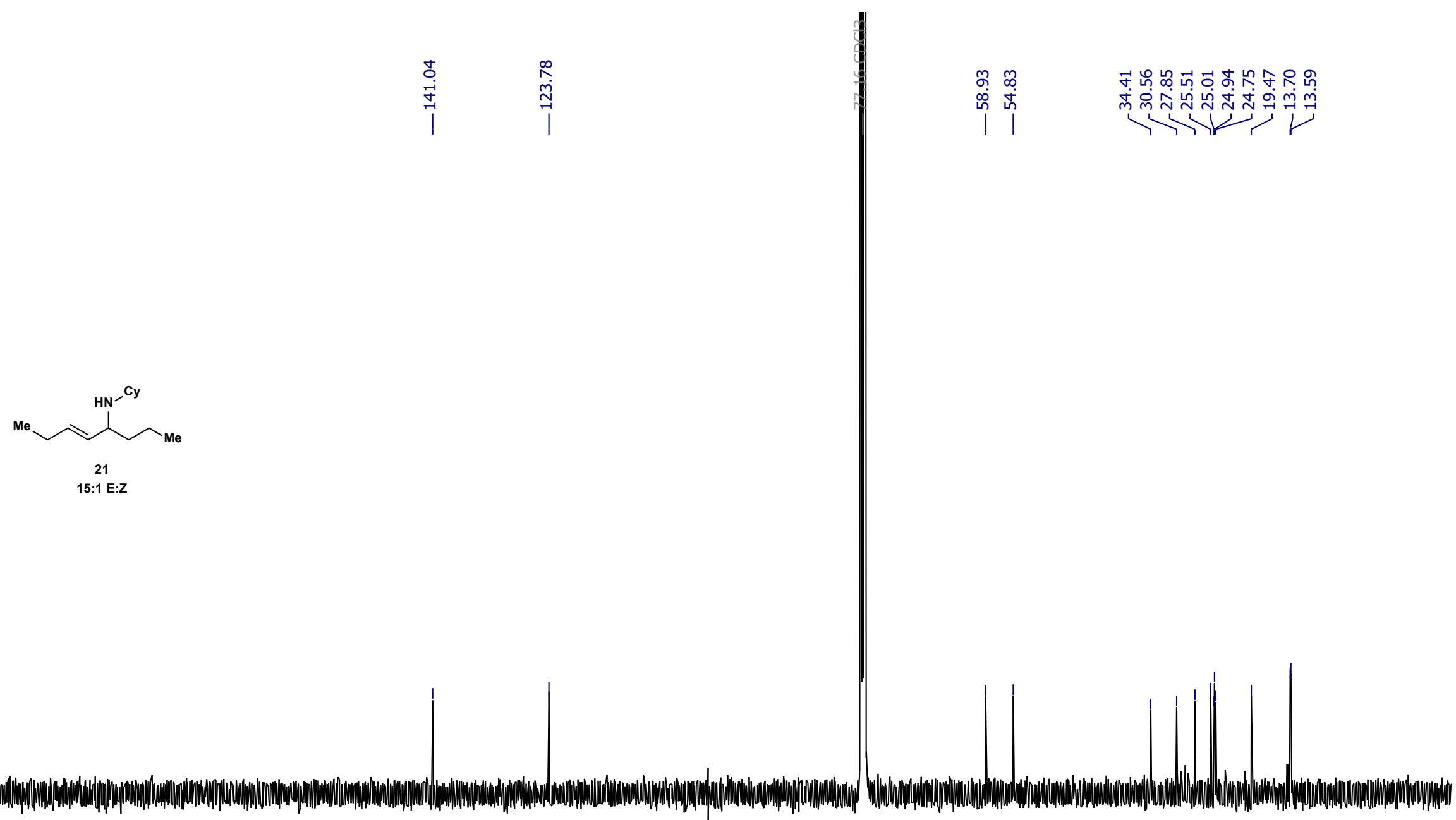

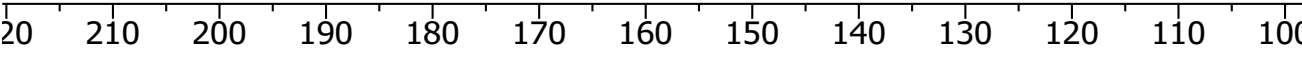
f1 (ppm) 
${ }^{1} \mathrm{H}$ NMR of 6-(cyclohexylamino)-3,7-dimethyloct-7-en-1-ol (22)

$\mathrm{CDCl}_{3}, 25^{\circ} \mathrm{C}$
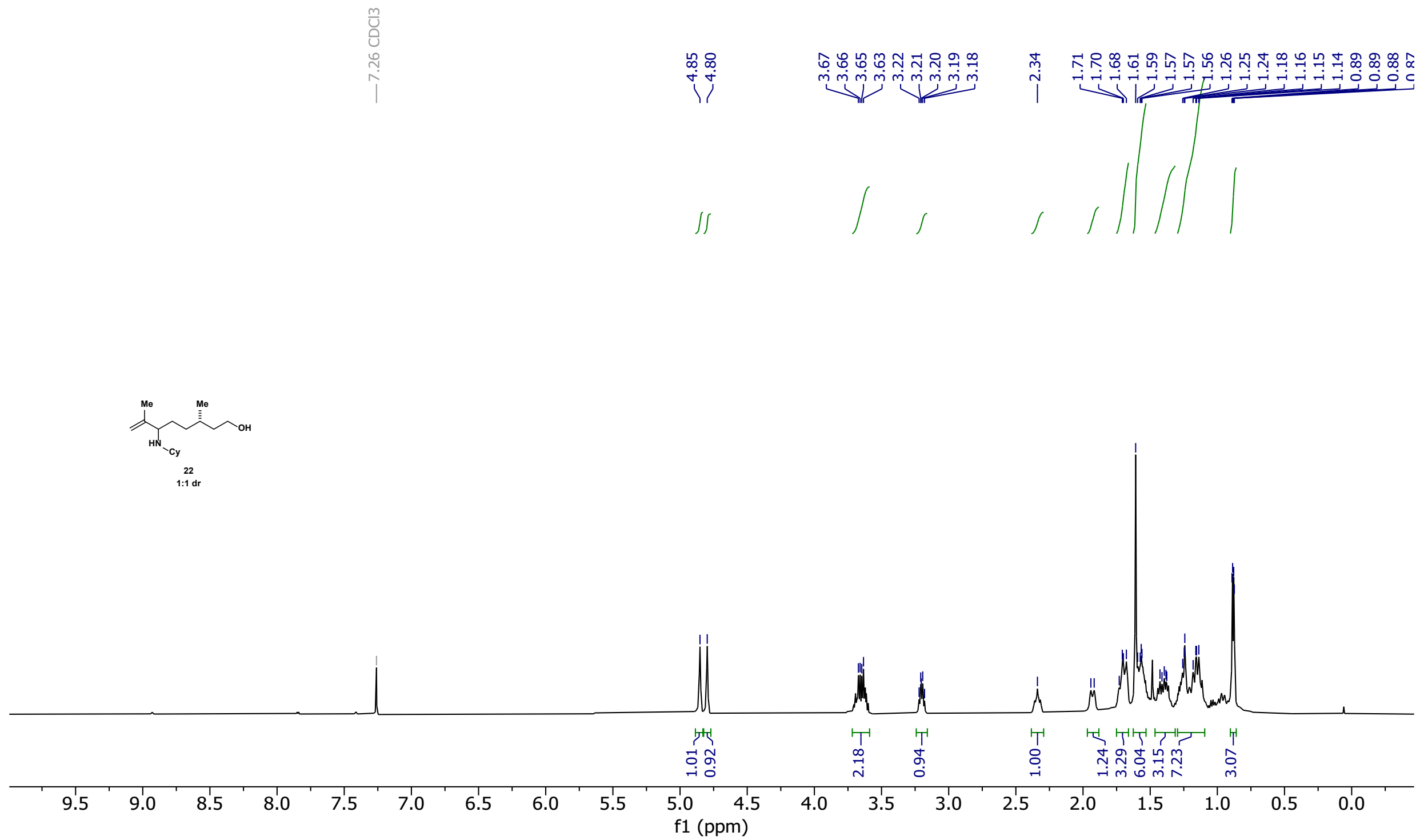
${ }^{13} \mathrm{C}$ NMR of 6-(cyclohexylamino)-3,7-dimethyloct-7-en-1-ol (22)

$\mathrm{CDCl}_{3}, 25^{\circ} \mathrm{C}$

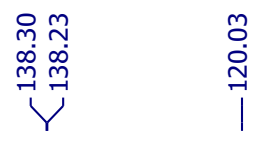

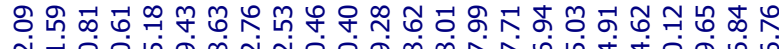

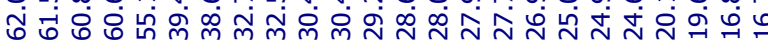

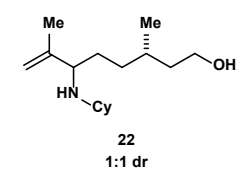

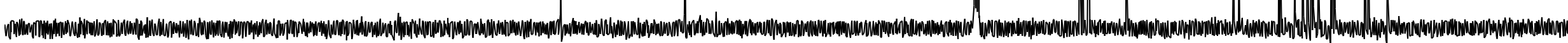

$\begin{array}{lllllllllllllllllllllllll}20 & 210 & 200 & 190 & 180 & 170 & 160 & 150 & 140 & 130 & 120 & \begin{array}{c}110 \\ \mathrm{f} 1(\mathrm{ppm})\end{array} & 90 & 80 & 70 & 60 & 50 & 40 & 30 & 20 & 10 & 0 & 1\end{array}$


${ }^{1} \mathrm{H}$ NMR of (Z)-N-(4-phenylbut-3-en-2-yl)cyclohexanamine (24)

$\mathrm{CDCl}_{3}, 25^{\circ} \mathrm{C}$
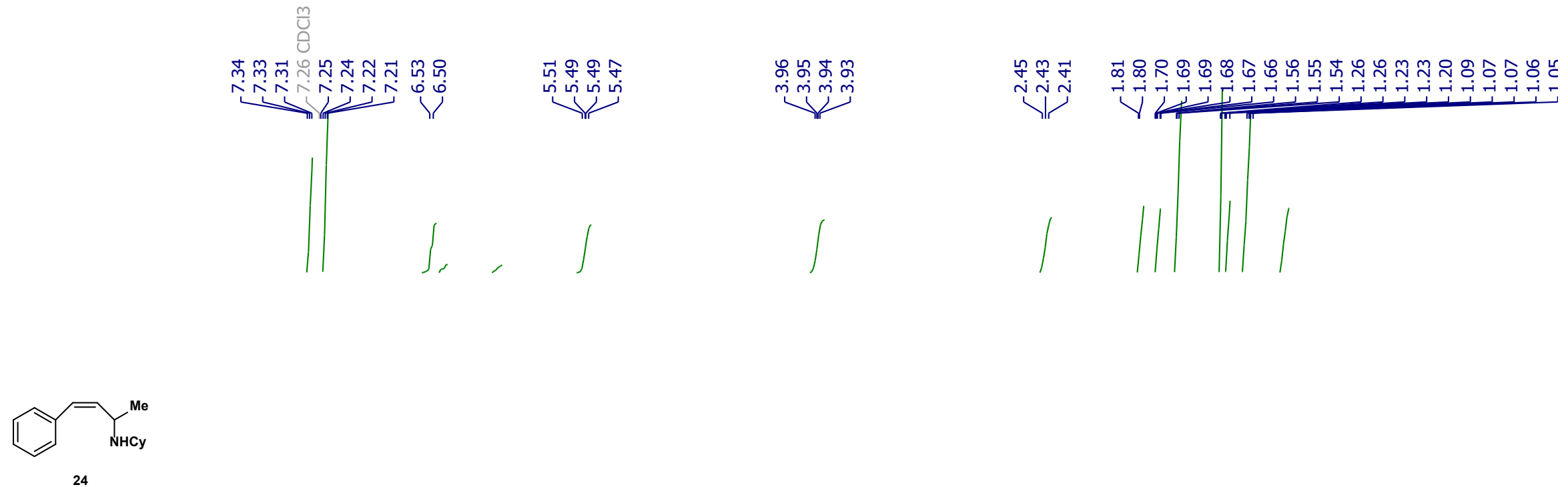

24
$1: 6 \mathrm{E}: Z$

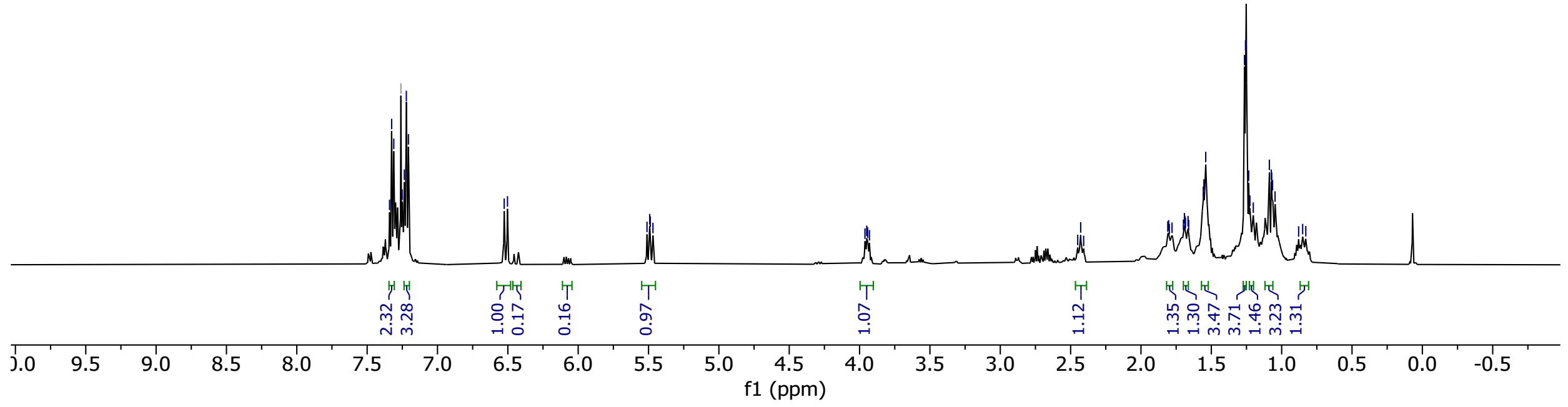


${ }^{13} \mathrm{C}$ NMR of (Z)-N-(4-phenylbut-3-en-2-yl)cyclohexanamine (24)

$\mathrm{CDCl}_{3}, 25^{\circ} \mathrm{C}$

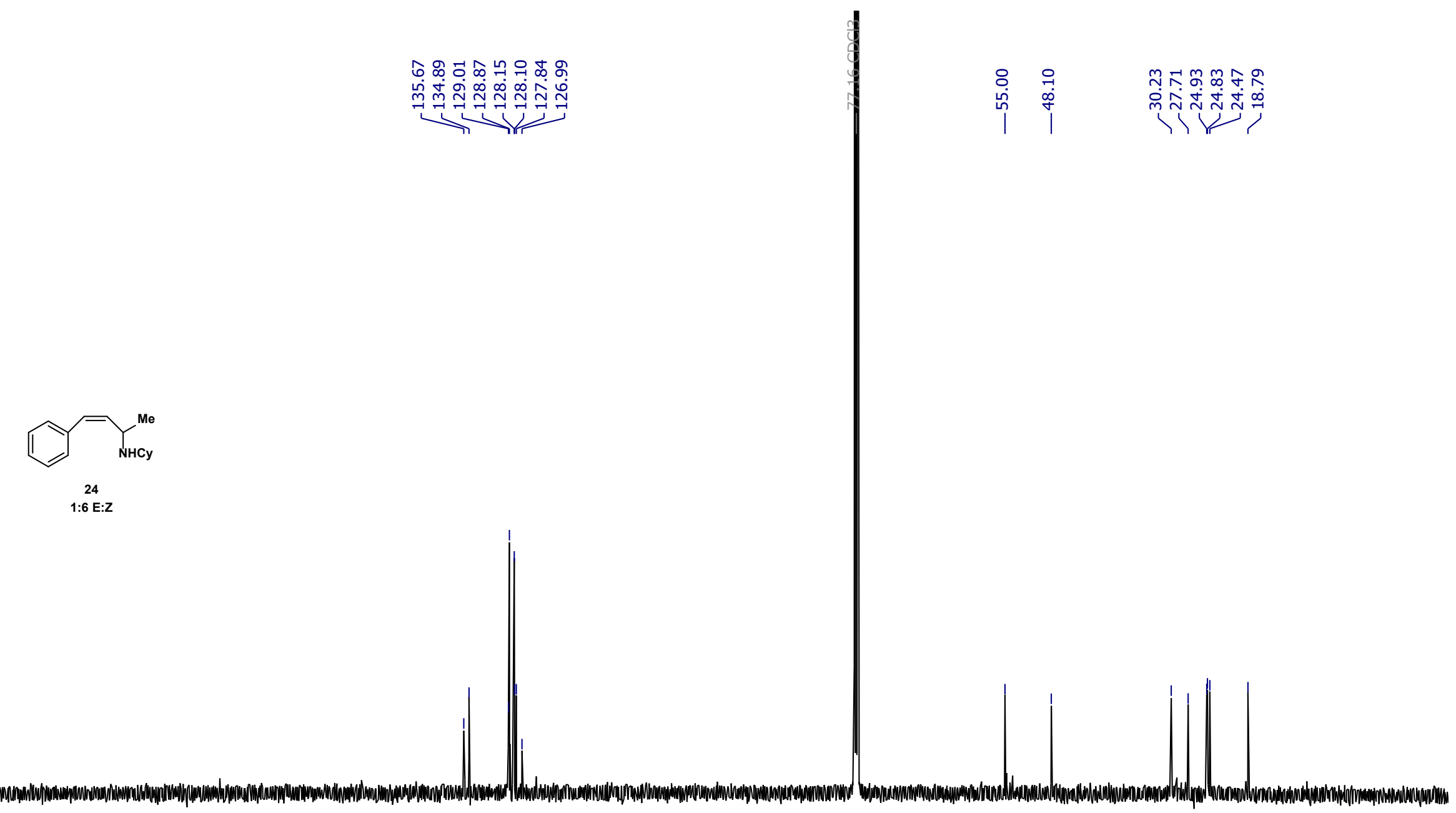

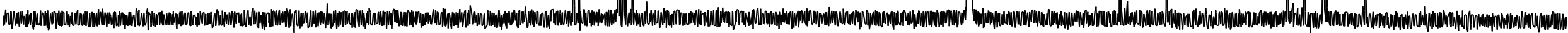

$\begin{array}{lllllllllllllllllllllll}210 & 200 & 190 & 180 & 170 & 160 & 150 & 140 & 130 & 120 & \begin{array}{c}110 \\ \mathrm{f} 1(\mathrm{ppm})\end{array} & 100 & 90 & 80 & 70 & 60 & 50 & 40 & 30 & 20 & 10 & 0 & -11\end{array}$


${ }^{1} \mathrm{H}$ NMR of $(E)-\mathrm{N}$-(oct-3-en-2-yl)cyclohexanamine (25)

$\mathrm{CDCl}_{3}, 25^{\circ} \mathrm{C}$

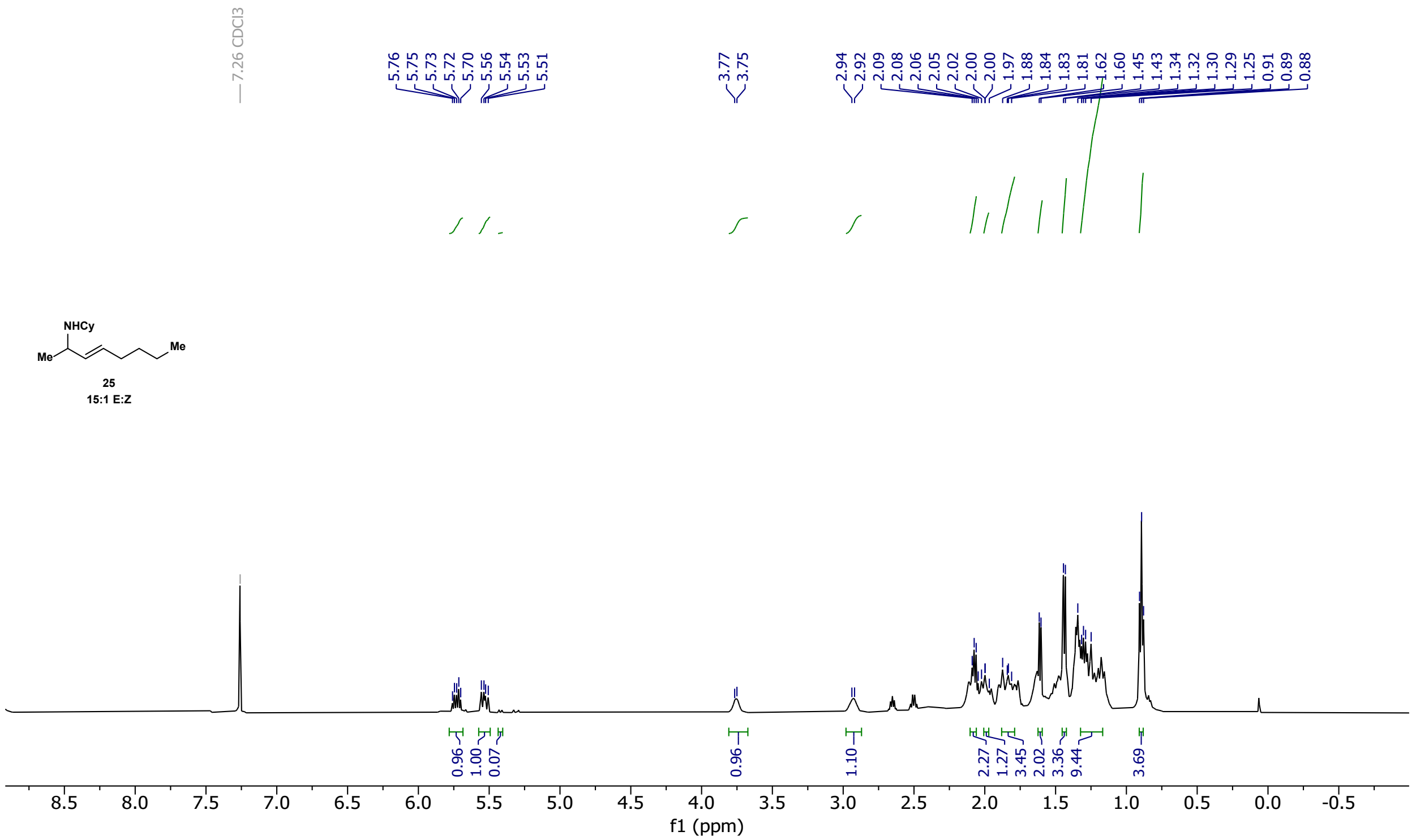


${ }^{13} \mathrm{C}$ NMR of (E)-N-(oct-3-en-2-yl)cyclohexanamine (25)

$\mathrm{CDCl}_{3}, 25^{\circ} \mathrm{C}$
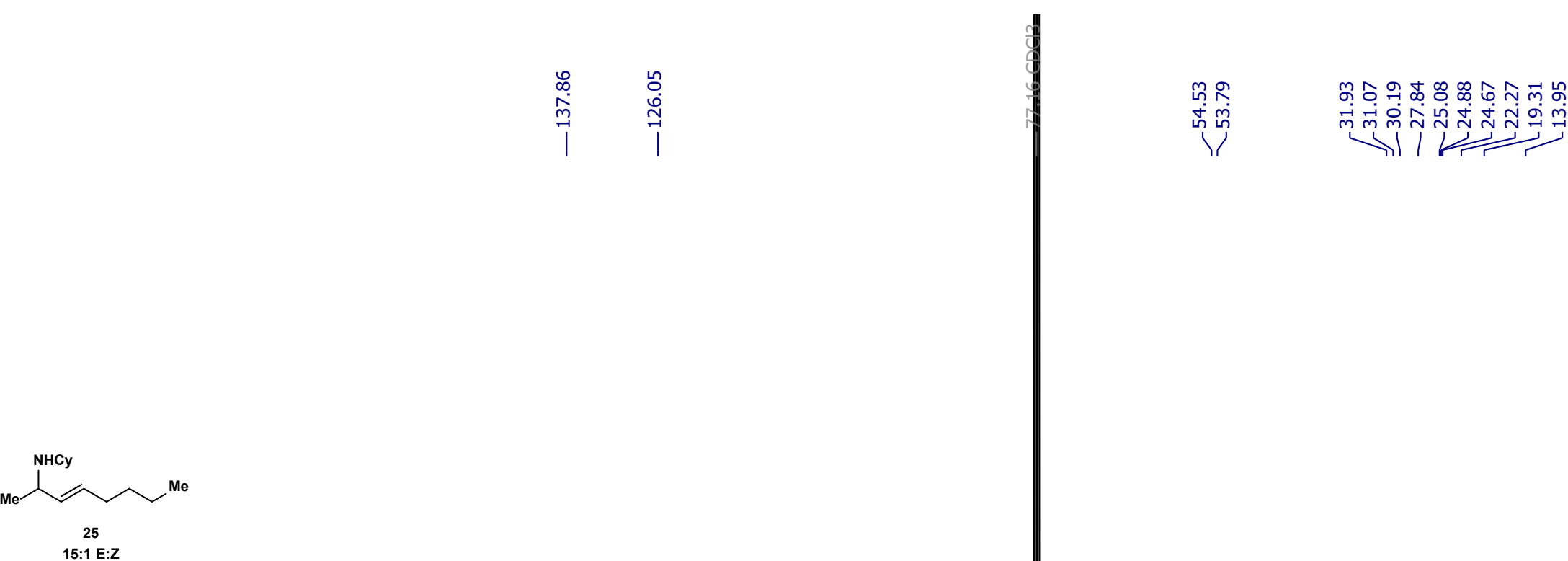

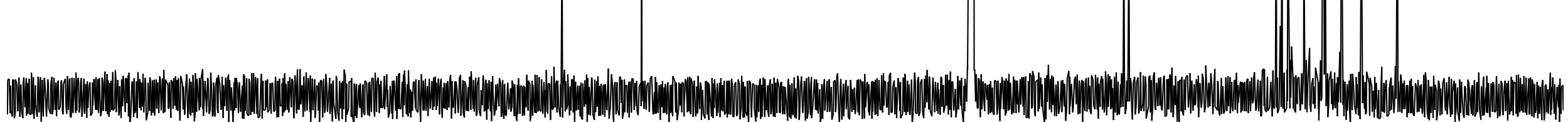

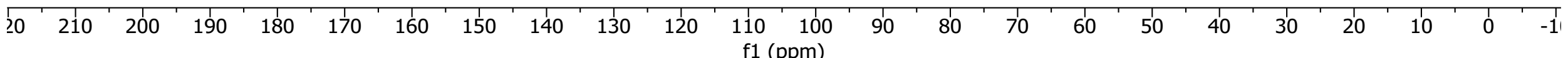




\section{${ }^{1} \mathrm{H}$ NMR of $\mathrm{N}$-(oct-1-en-3-yl)cyclohexanamine (25')}

$\mathrm{CDCl}_{3}, 25^{\circ} \mathrm{C}$
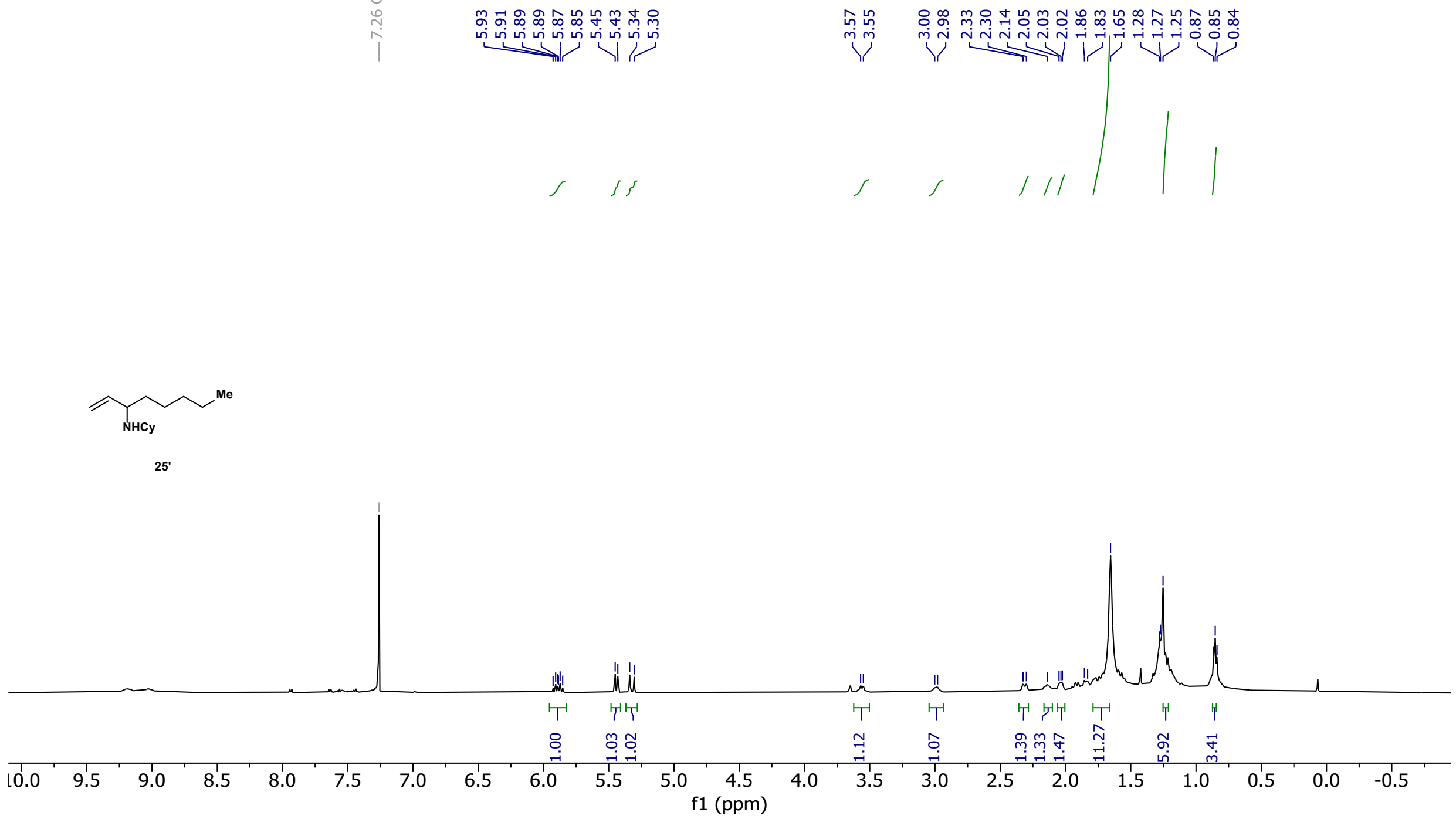
${ }^{1} \mathrm{H}$ NMR of (E)-6-(cyclohexylamino)-3,7-dimethylocta-2,7-dien-1-yl acetate (26)

$\mathrm{CDCl}_{3}, 25^{\circ} \mathrm{C}$

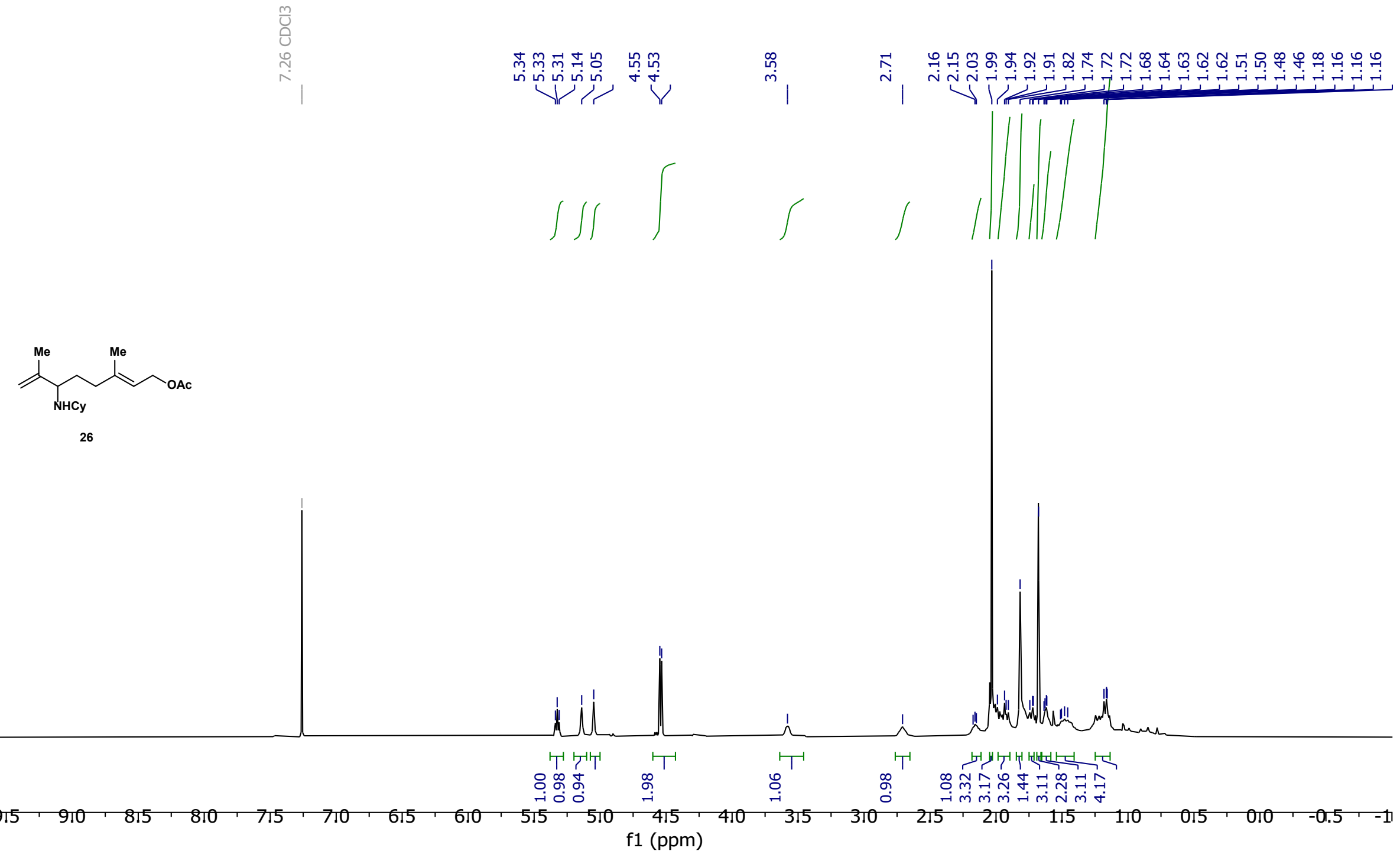


${ }^{13} \mathrm{C}$ NMR of (E)-6-(cyclohexylamino)-3,7-dimethylocta-2,7-dien-1-yl acetate (26)

$\mathrm{CDCl}_{3}, 25^{\circ} \mathrm{C}$

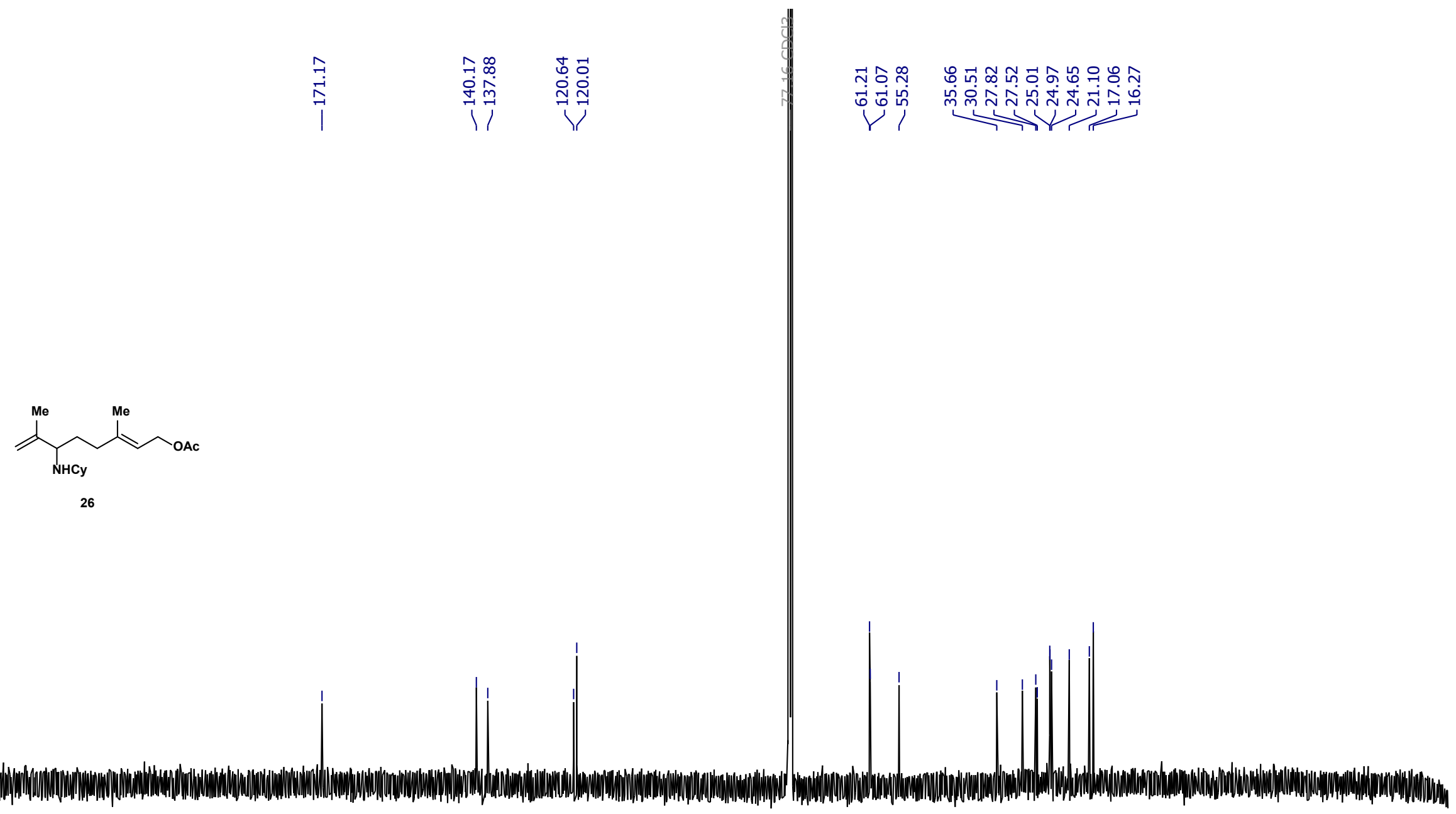

$\begin{array}{llllllllllllllllllllllllllllllll}1 & 250 & 240 & 230 & 220 & 210 & 200 & 190 & 180 & 170 & 160 & 150 & 140 & 130 & 120 & 110 & 100 & 90 & 80 & 70 & 60 & 50 & 40 & 30 & 20 & 10 & 0 & -10 & -20 & -30 & -40 & -50\end{array}$ f1 (ppm) 
${ }^{1} \mathrm{H}$ NMR of $(E)-3,7-d i m e t h y l o c t a-2,6-d i e n-1-y l$ acetate (27)

$\mathrm{CDCl}_{3}, 25^{\circ} \mathrm{C}$

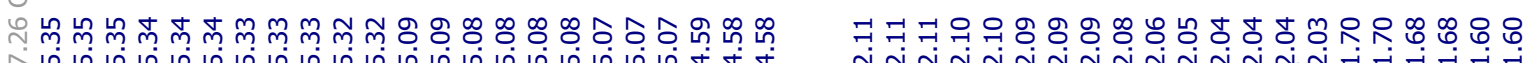

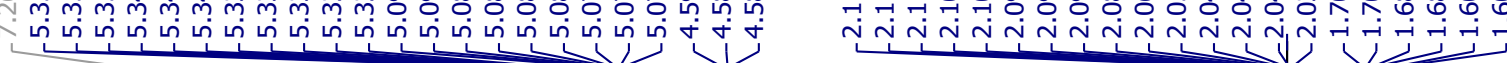

$\overbrace{27}^{M e} \overbrace{M e}^{\text {Me }}$

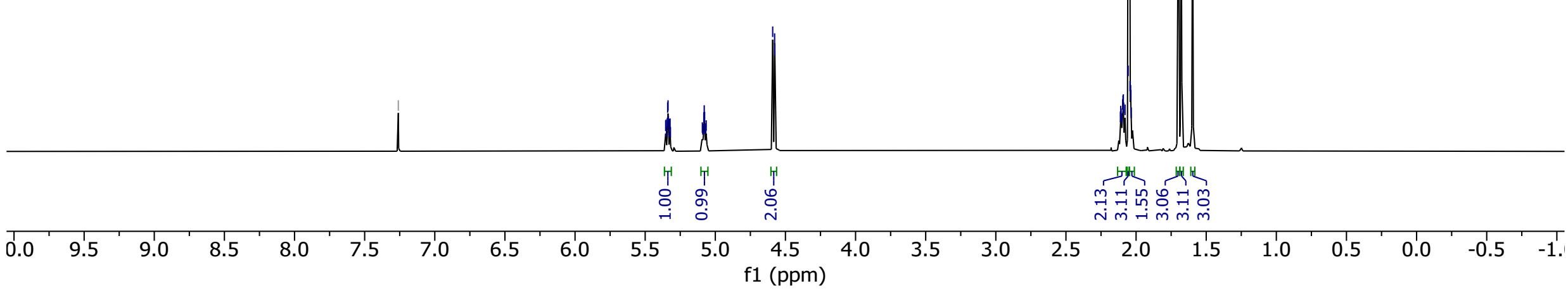


${ }^{13} \mathrm{C}$ NMR of (E)-3,7-dimethylocta-2,6-dien-1-yl acetate (27)

$\mathrm{CDCl}_{3}, 25^{\circ} \mathrm{C}$

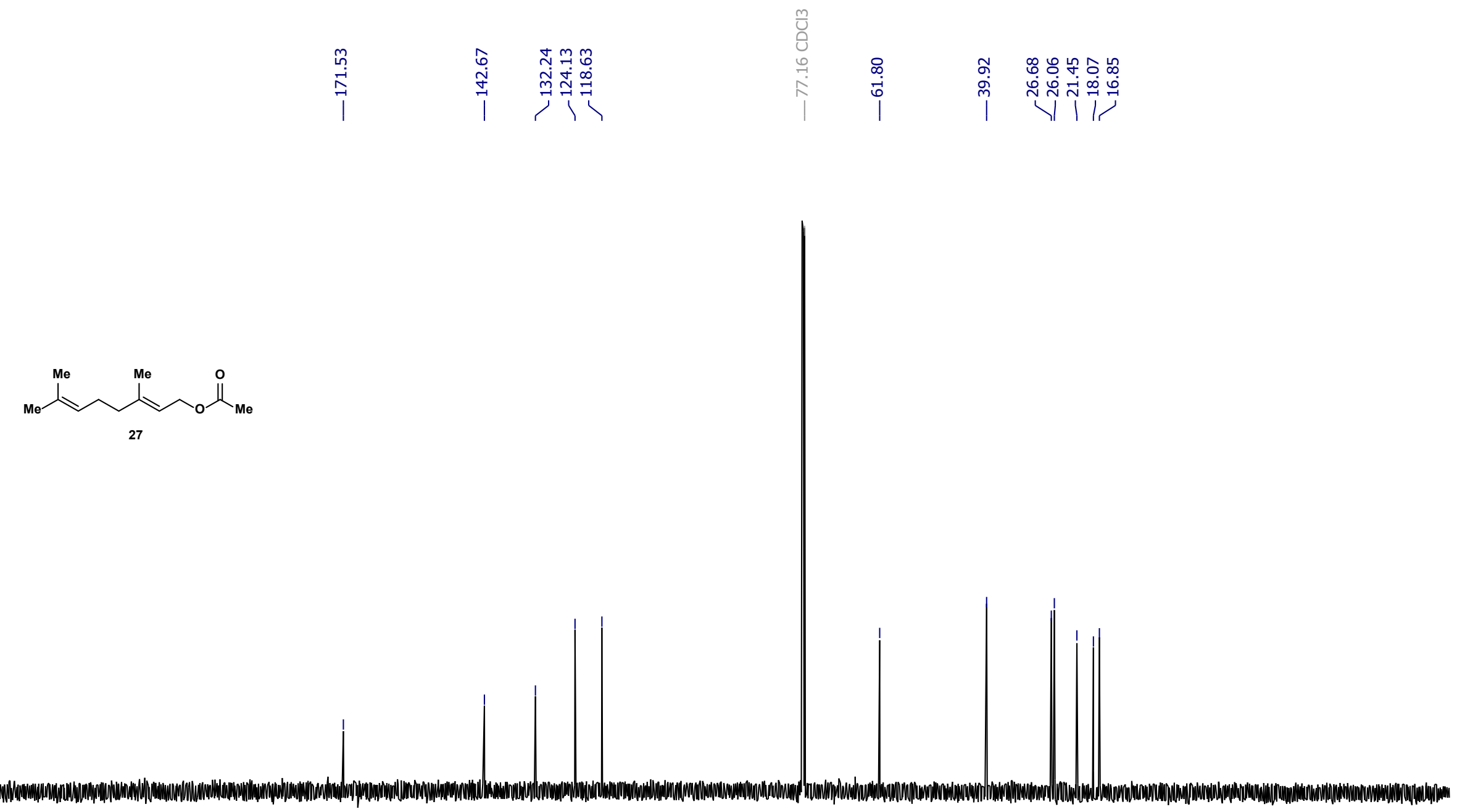

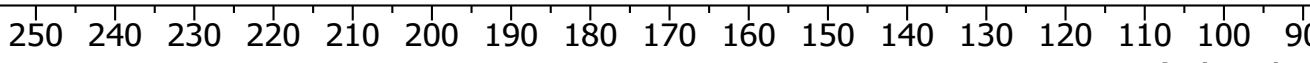

f1 (ppm) 


\section{${ }^{1} \mathrm{H}$ NMR of 4-(cyclohexylamino)allylestrenol (28)}

$\mathrm{CDCl}_{3}, 55^{\circ} \mathrm{C}$

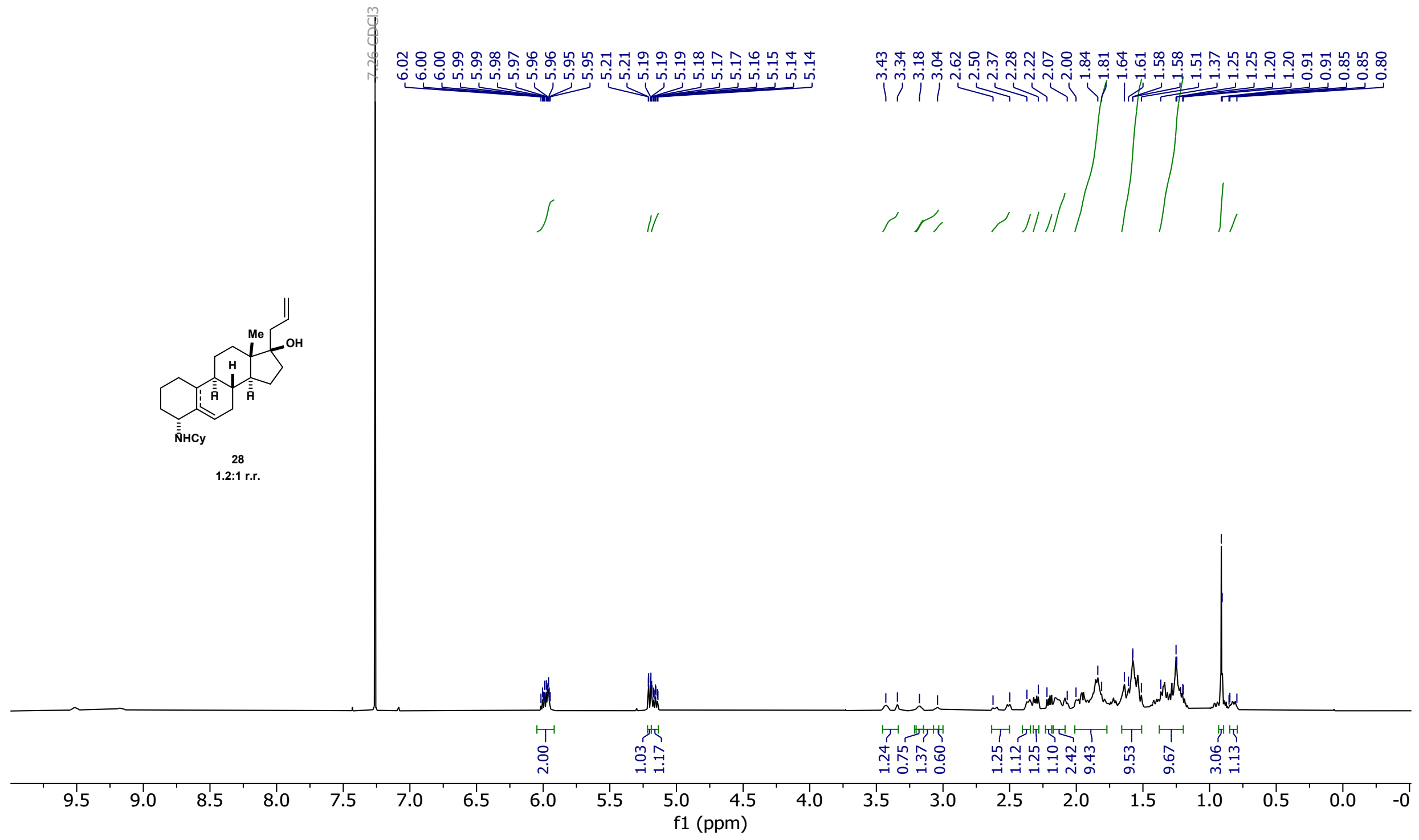


${ }^{13} \mathrm{C}$ NMR of 4-(cyclohexylamino)allylestrenol (28)

$\mathrm{CDCl}_{3}, 55^{\circ} \mathrm{C}$
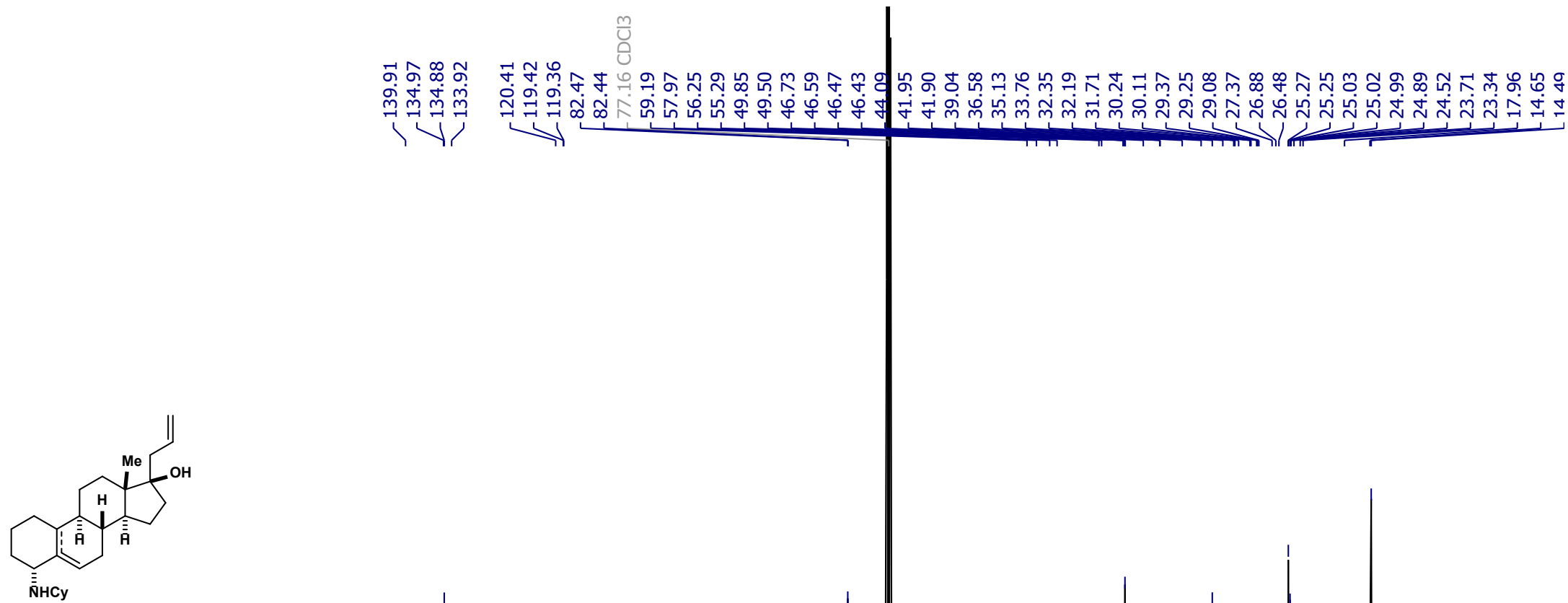

28
$1.2: 1$ r.r.

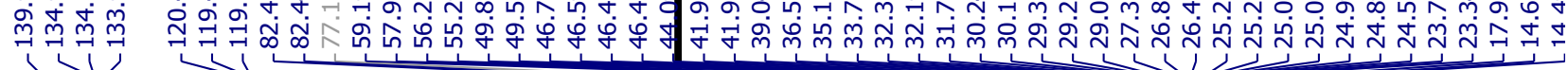

$\begin{array}{llllllllllllllllllllllll}\mid & 110 & 200 & 190 & 180 & 170 & 160 & 150 & 140 & 130 & 120 & \begin{array}{c}110 \\ \mathrm{f} 1(\mathrm{ppm})\end{array} & 90 & 80 & 70 & 60 & 50 & 40 & 30 & 20 & 10 & 0 & -1\end{array}$


${ }^{1} \mathrm{H}-{ }^{1} \mathrm{H}$ COSY spectrum of 4-(cyclohexylamino)allylestrenol (28)

$\mathrm{CDCl}_{3}, 55^{\circ} \mathrm{C}$

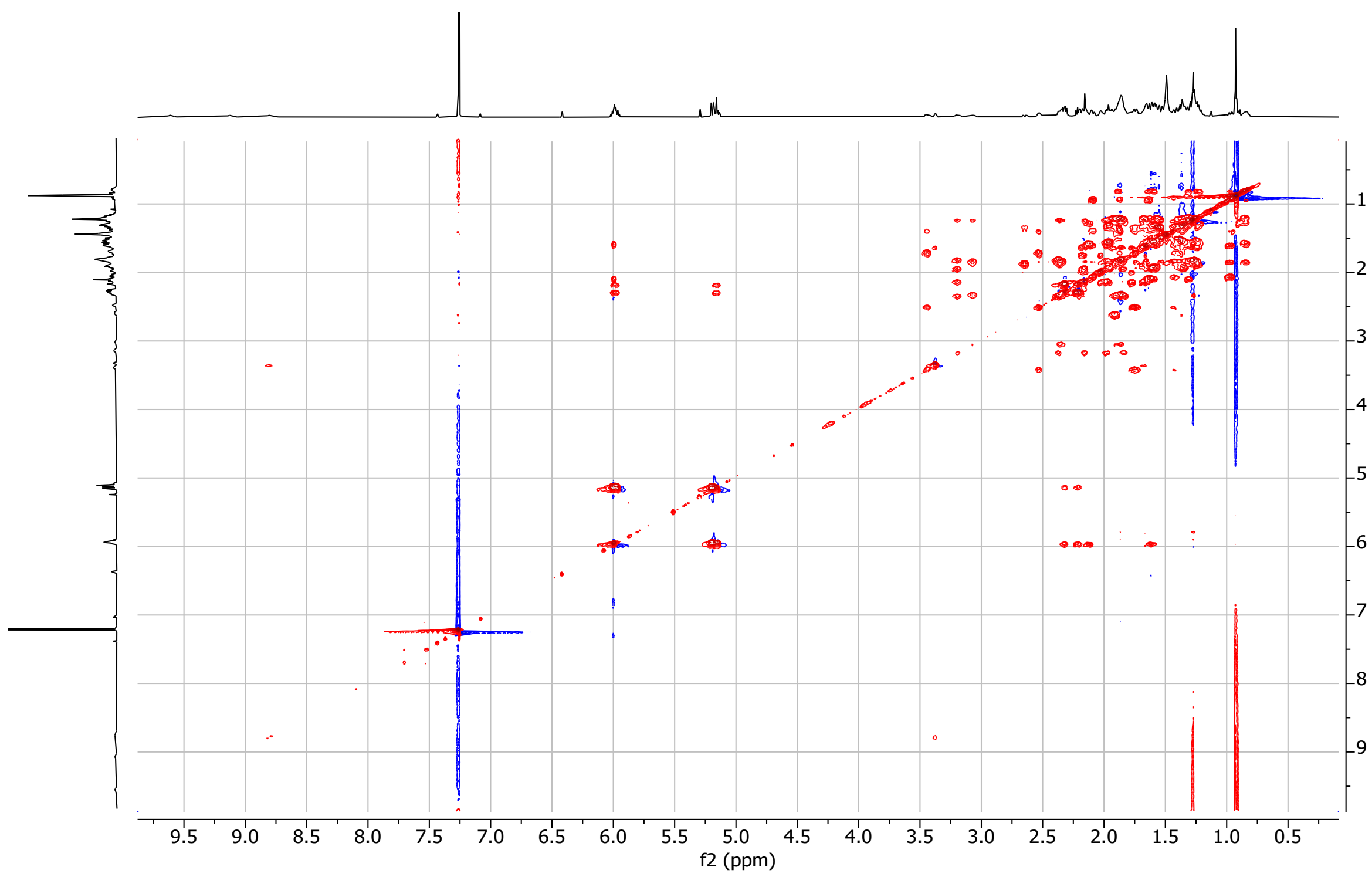


${ }^{1} \mathrm{H}-{ }^{13} \mathrm{C}$ HSQC spectrum of 4-(cyclohexylamino)allylestrenol (28)

$\mathrm{CDCl}_{3}, 55^{\circ} \mathrm{C}$

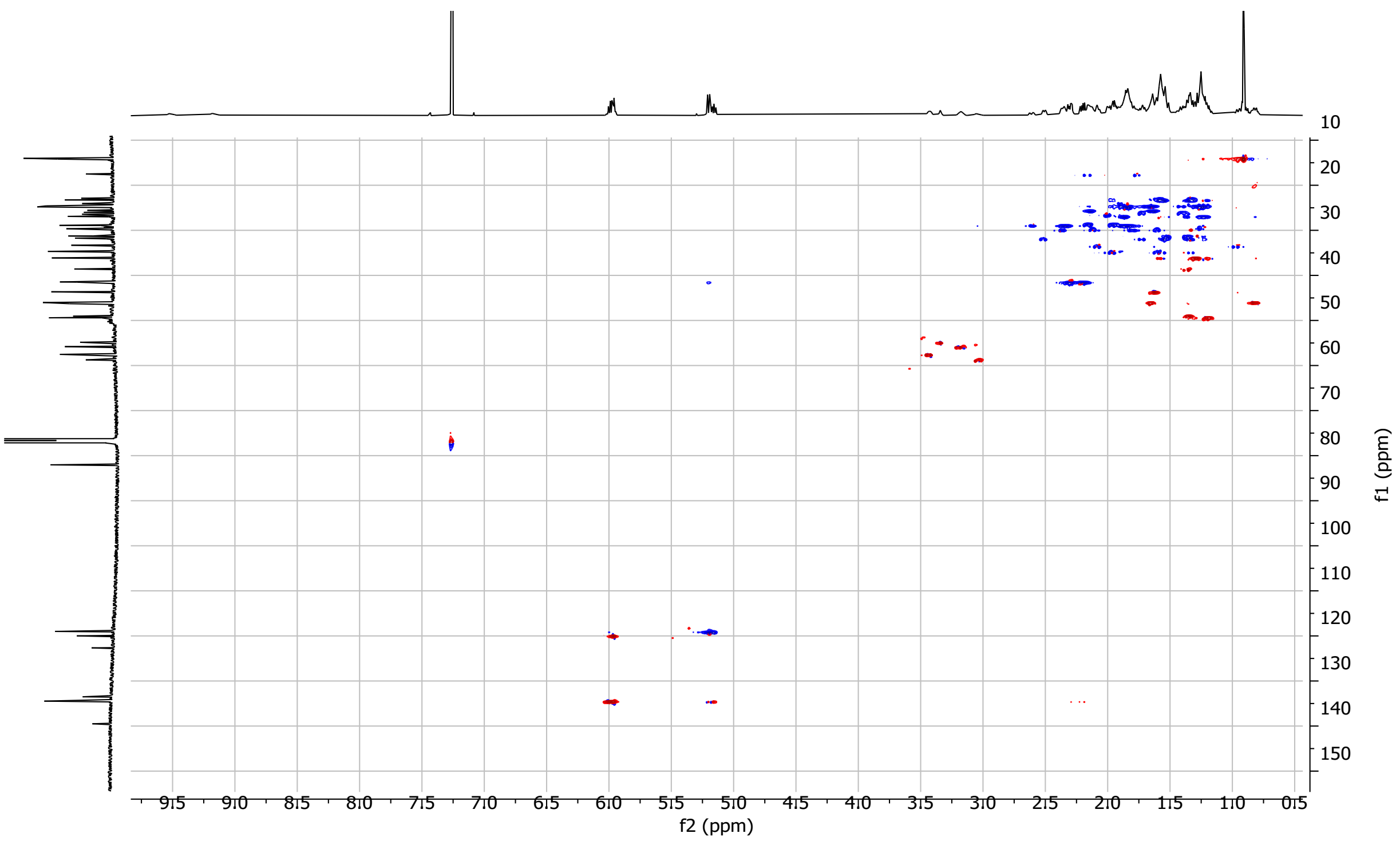


${ }^{1} \mathrm{H}-{ }^{13} \mathrm{C}$ HMBC spectrum of 4-(cyclohexylamino)allylestrenol (28)

$\mathrm{CDCl}_{3}, 55^{\circ} \mathrm{C}$

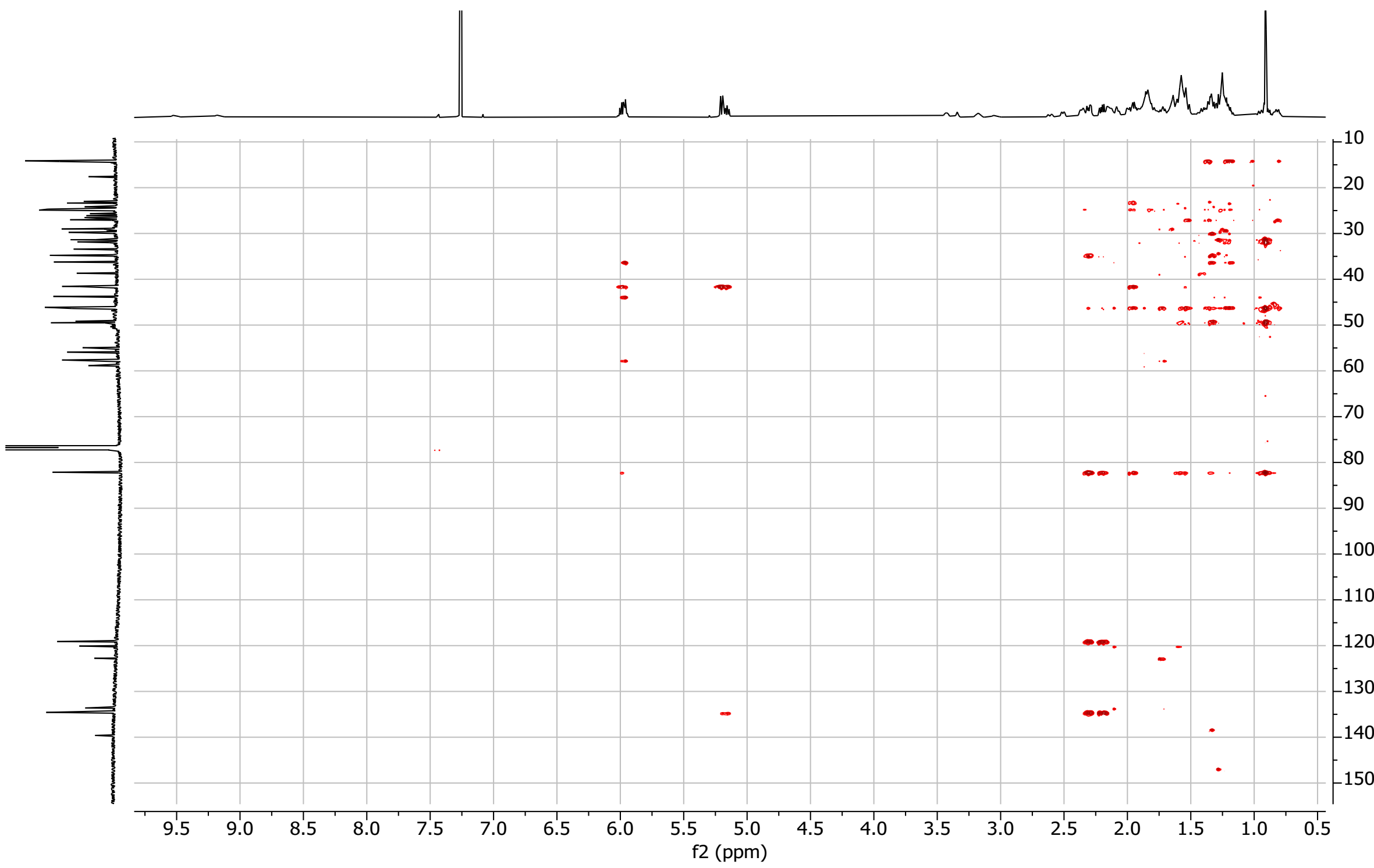


SUPPORTING INFORMATION

S143

${ }^{1} \mathrm{H}-{ }^{1} \mathrm{H}$ NOESY spectrum of 4-(cyclohexylamino)allylestrenol (28)

$\mathrm{CDCl}_{3}, 55^{\circ} \mathrm{C}$

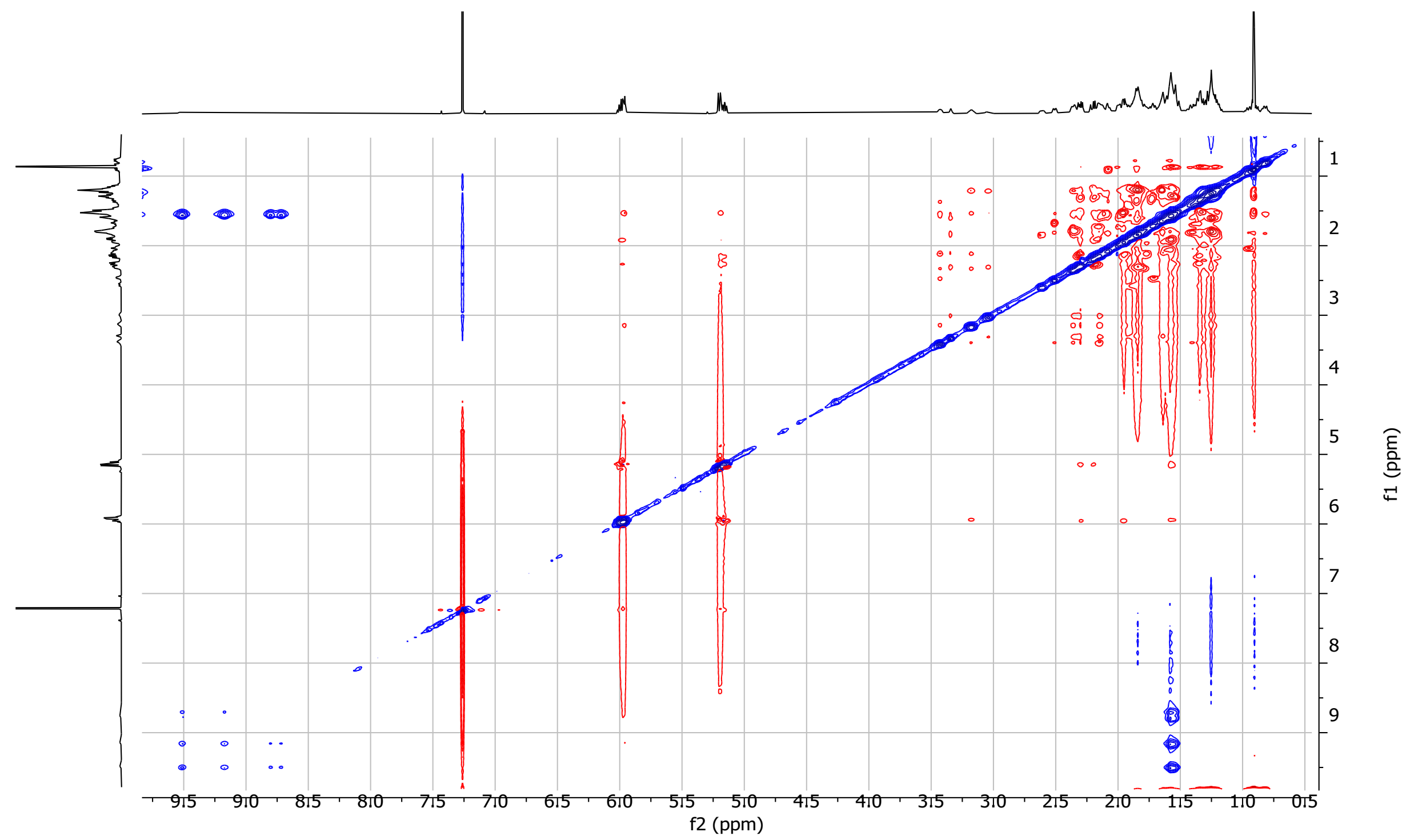


${ }^{1} \mathrm{H}$ NMR of epiandrosterone-derivative (30)

$\mathrm{CDCl}_{3}, 25^{\circ} \mathrm{C}$

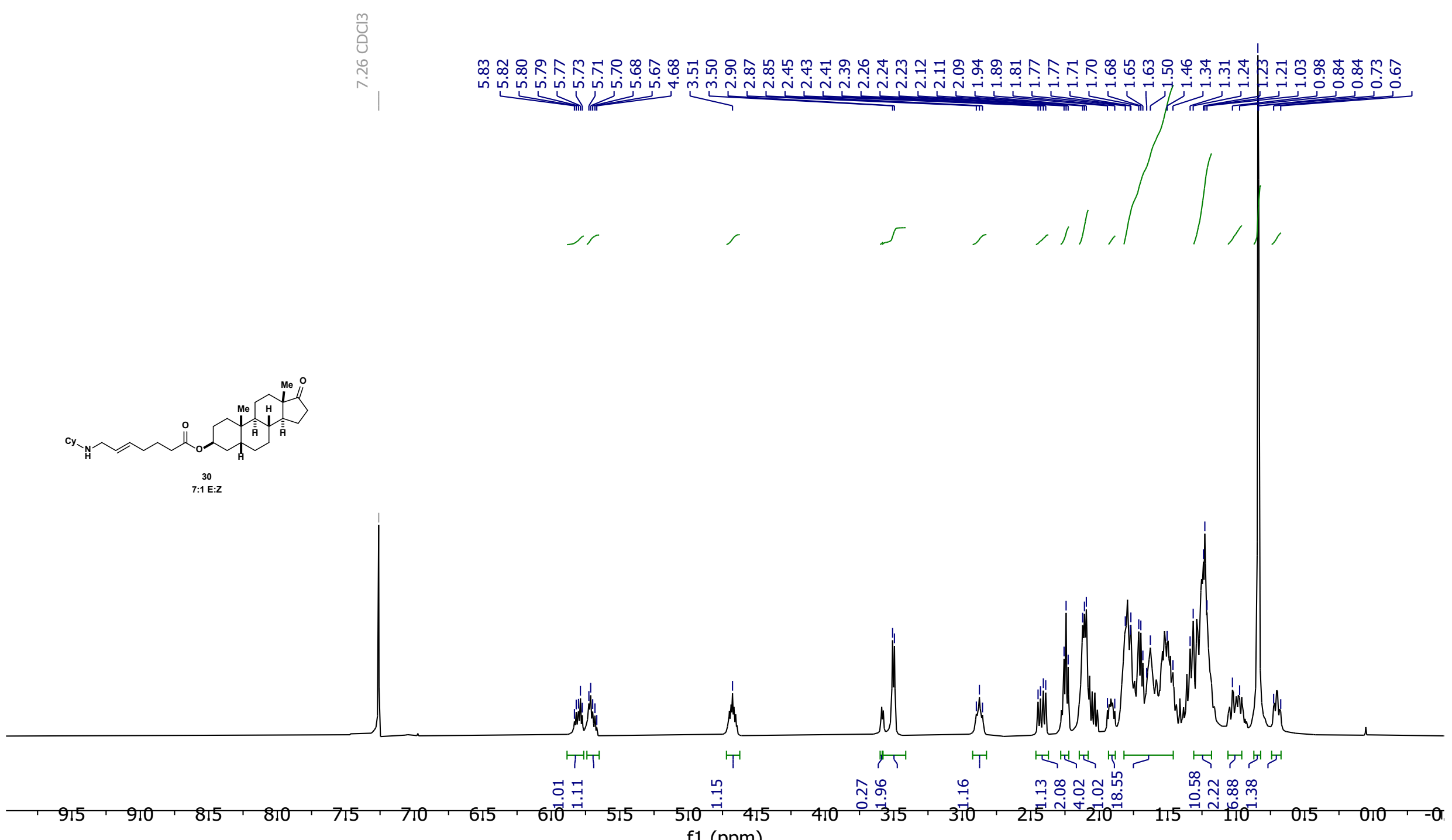


${ }^{13} \mathrm{C}$ NMR of epiandrosterone-derivative (30)

$\mathrm{CDCl}_{3}, 25^{\circ} \mathrm{C}$

$\stackrel{\stackrel{n}{i}}{\stackrel{\sim}{N}}$

$\stackrel{\overrightarrow{9}}{\stackrel{5}{N}}$

员

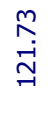

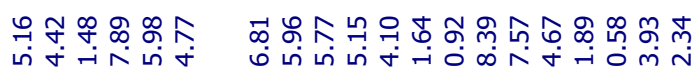

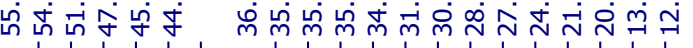

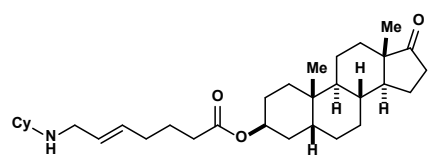

7:1::z

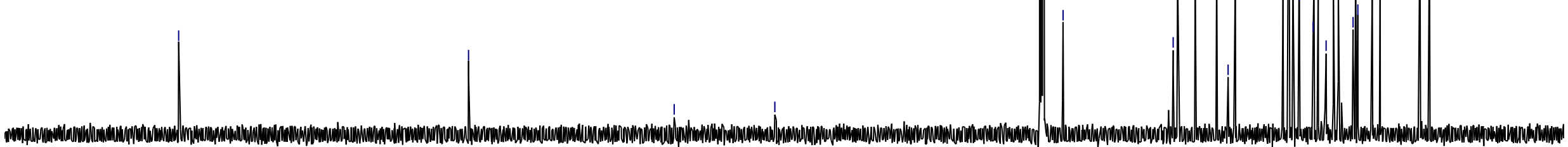

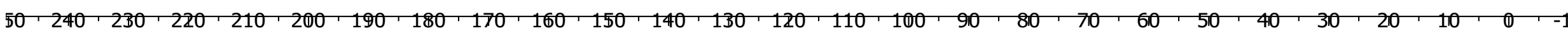
f1 (ppm) 
${ }^{1} \mathrm{H}$ NMR of (Z)-N-(tert-butyl)-3-phenyl-2-propenamine (31)

$\mathrm{CDCl}_{3}, 25^{\circ} \mathrm{C}$
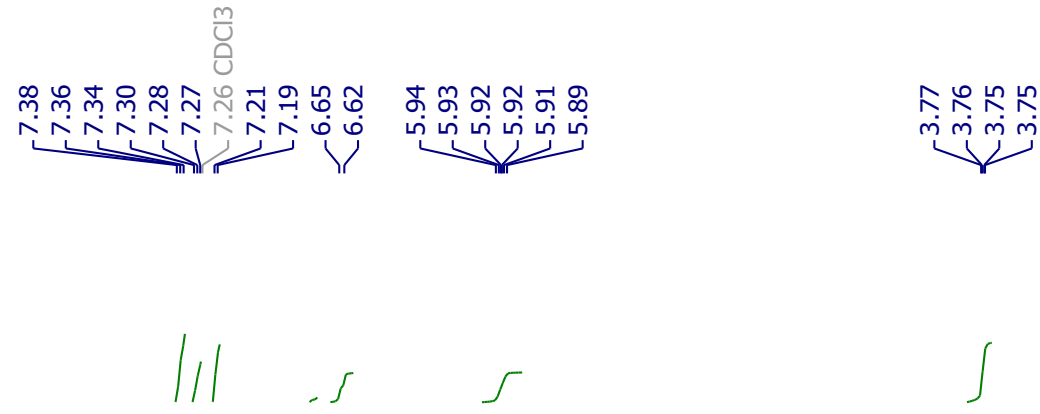

幽

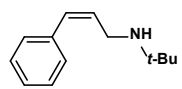

31
$1: 6: 2$
13

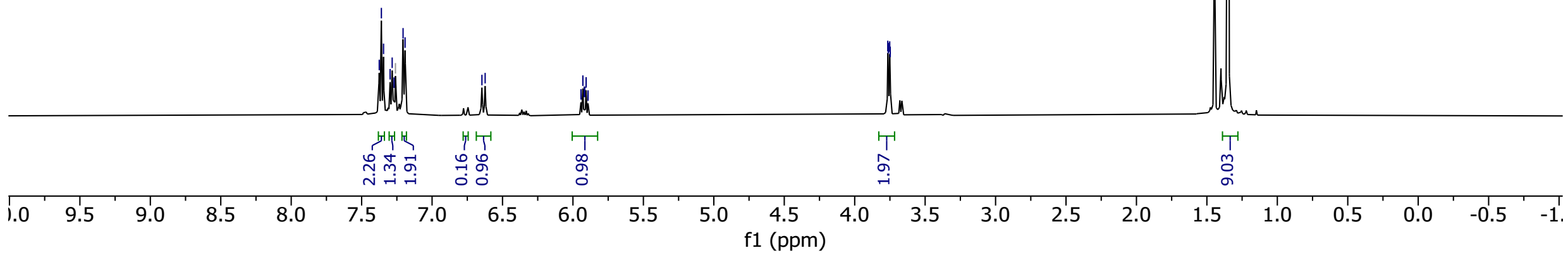


${ }^{13} \mathrm{C}$ NMR of (Z)-N-(tert-butyl)-3-phenyl-2-propenamine (31)

$\mathrm{CDCl}_{3}, 25^{\circ} \mathrm{C}$

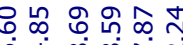

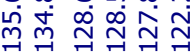
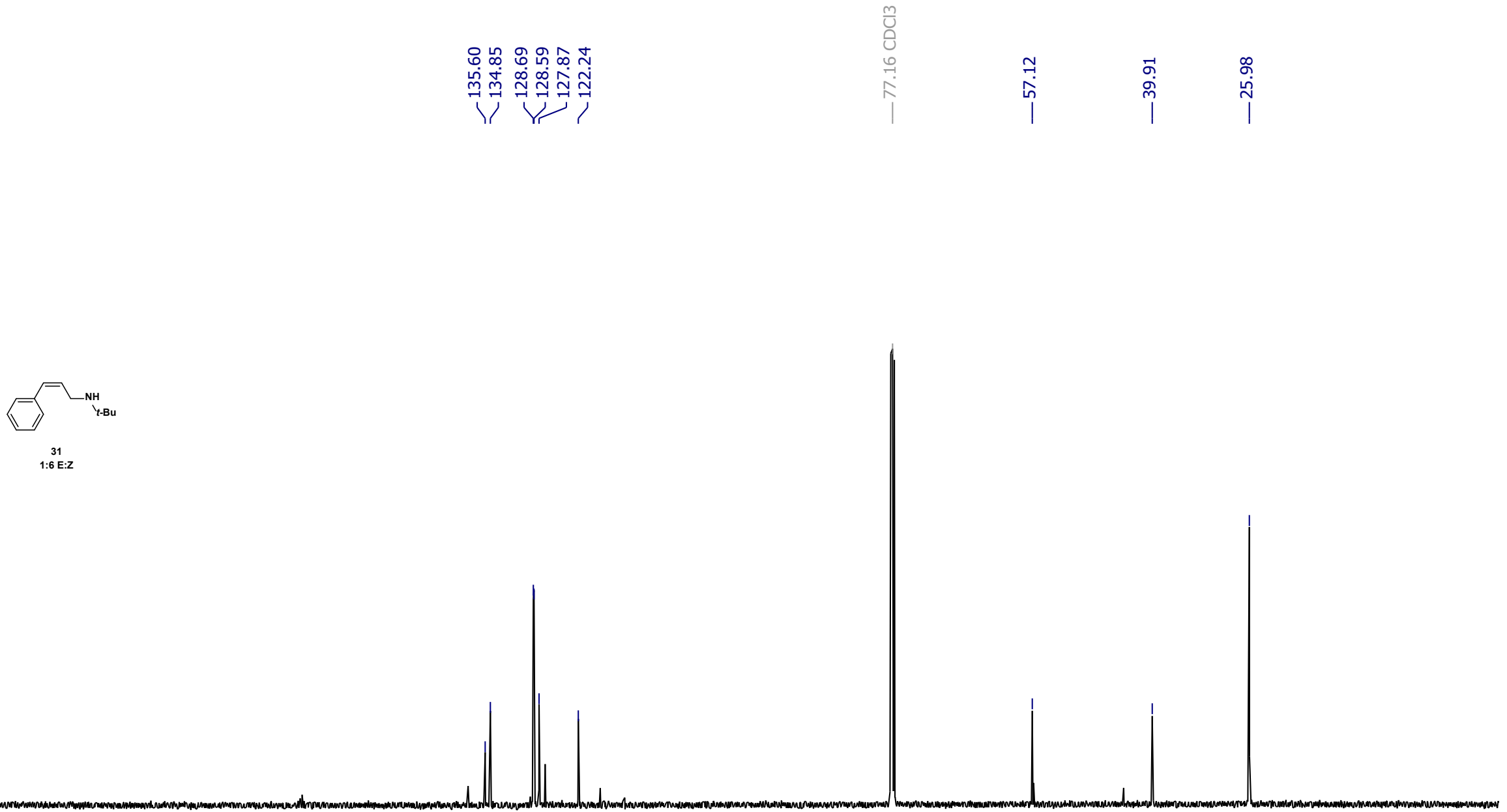

$\begin{array}{lllllllllllll}20 & 210 & 200 & 190 & 180 & 170 & 160 & 150 & 140 & 130 & 120 & 110 & 100 \\ \mathrm{f} 1(\mathrm{ppm})\end{array}$

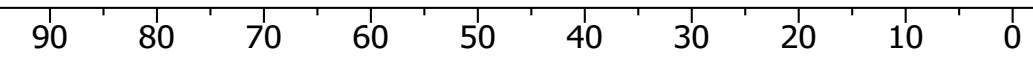


${ }^{1} \mathrm{H}$ NMR of (Z)-N-(tert-butyl)-2-cyclooctenamine (32)

$\mathrm{CDCl}_{3}, 25^{\circ} \mathrm{C}$

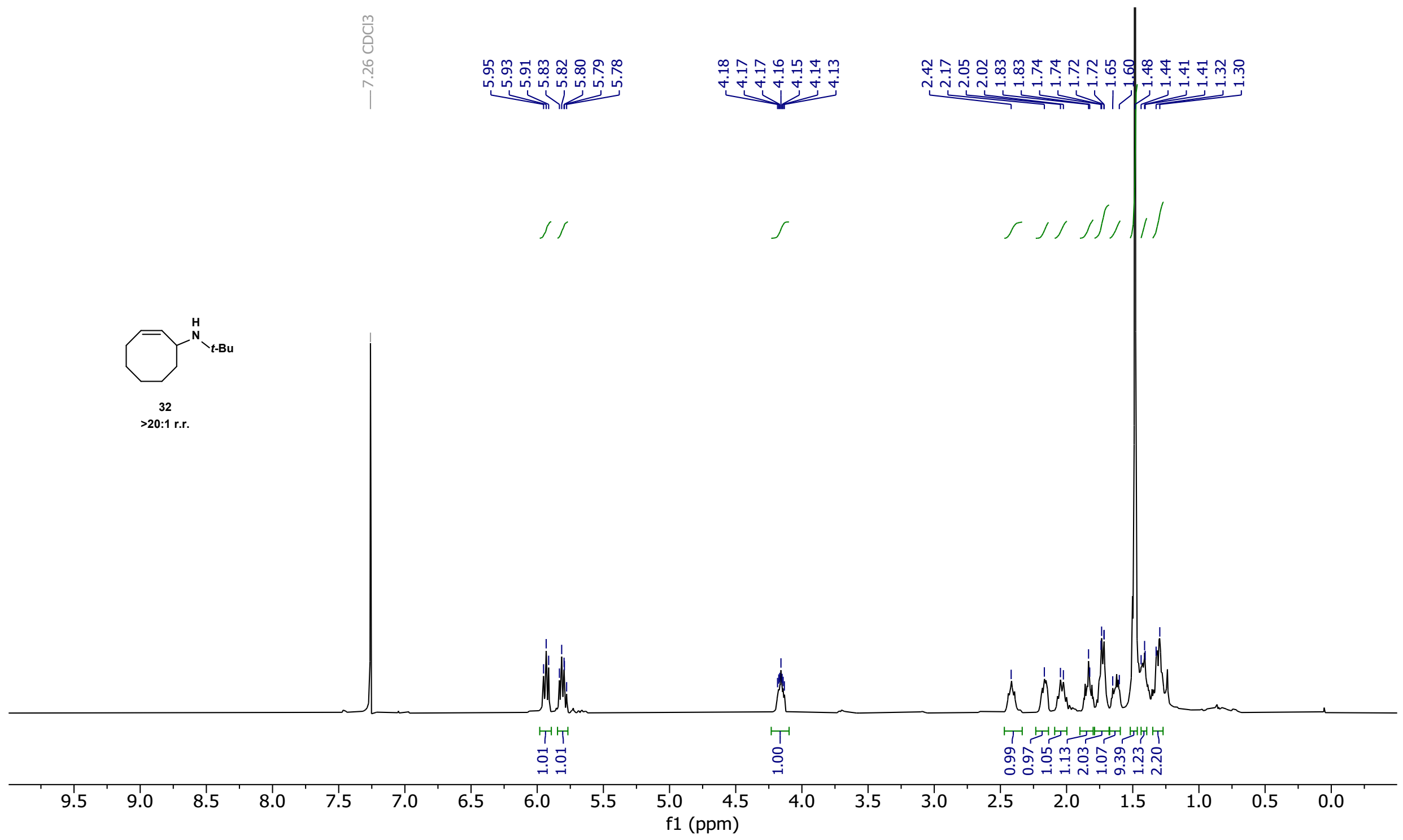


${ }^{13} \mathrm{C}$ NMR of (Z)-N-(tert-butyl)-2-cyclooctenamine (32)

$\mathrm{CDCl}_{3}, 25^{\circ} \mathrm{C}$

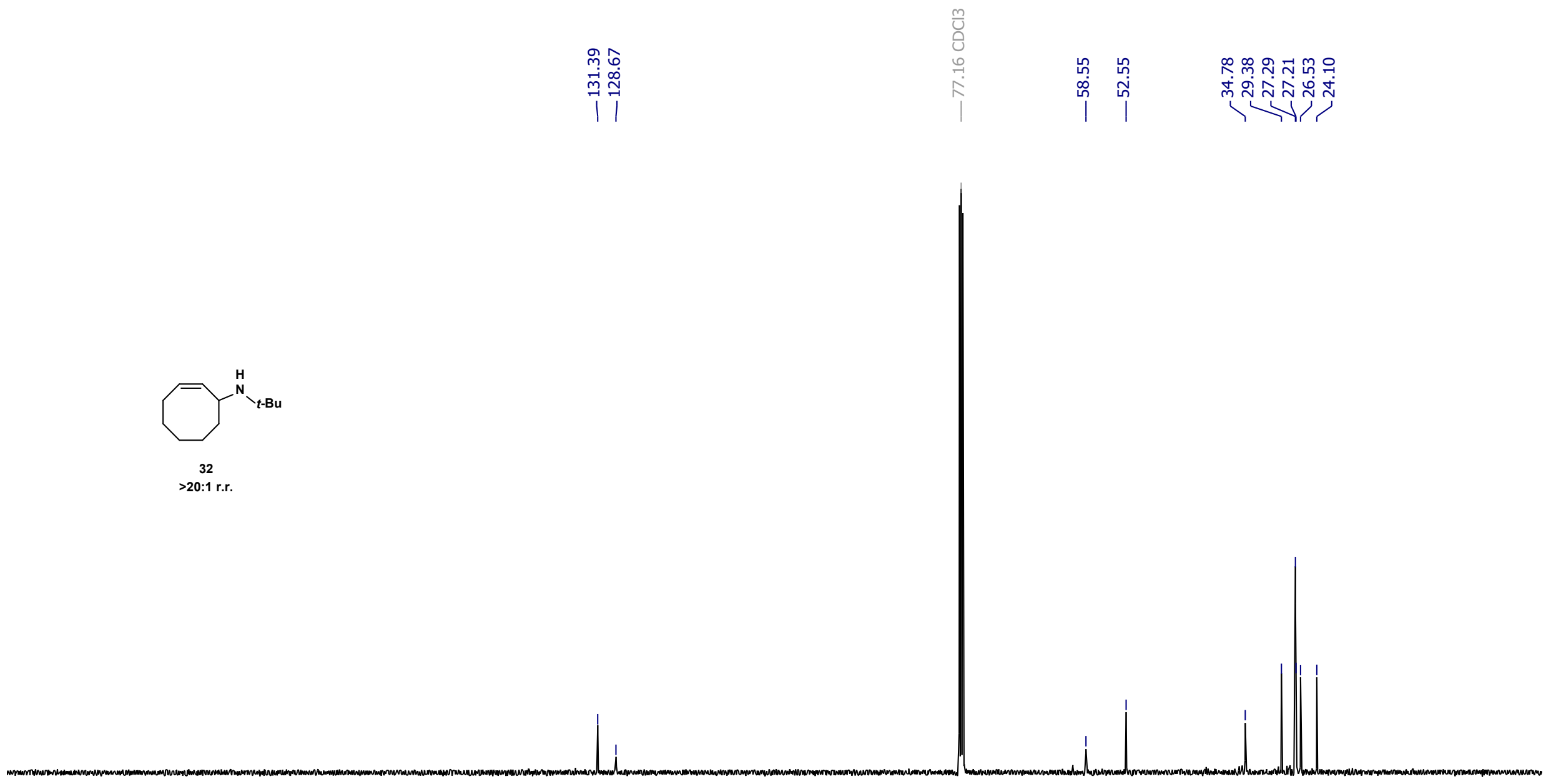

$\begin{array}{llllllllllll}210 & 200 & 190 & 180 & 170 & 160 & 150 & 140 & 130 & 120 & 110 & 100 \\ \mathrm{f} 1(\mathrm{ppm})\end{array}$

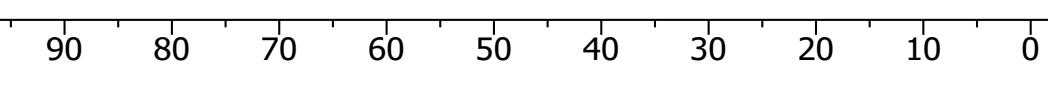


${ }^{1} \mathrm{H}$ NMR of (Z)-N-propyl-2-cyclooctenamine (33)

$\mathrm{CDCl}_{3}, 25^{\circ} \mathrm{C}$
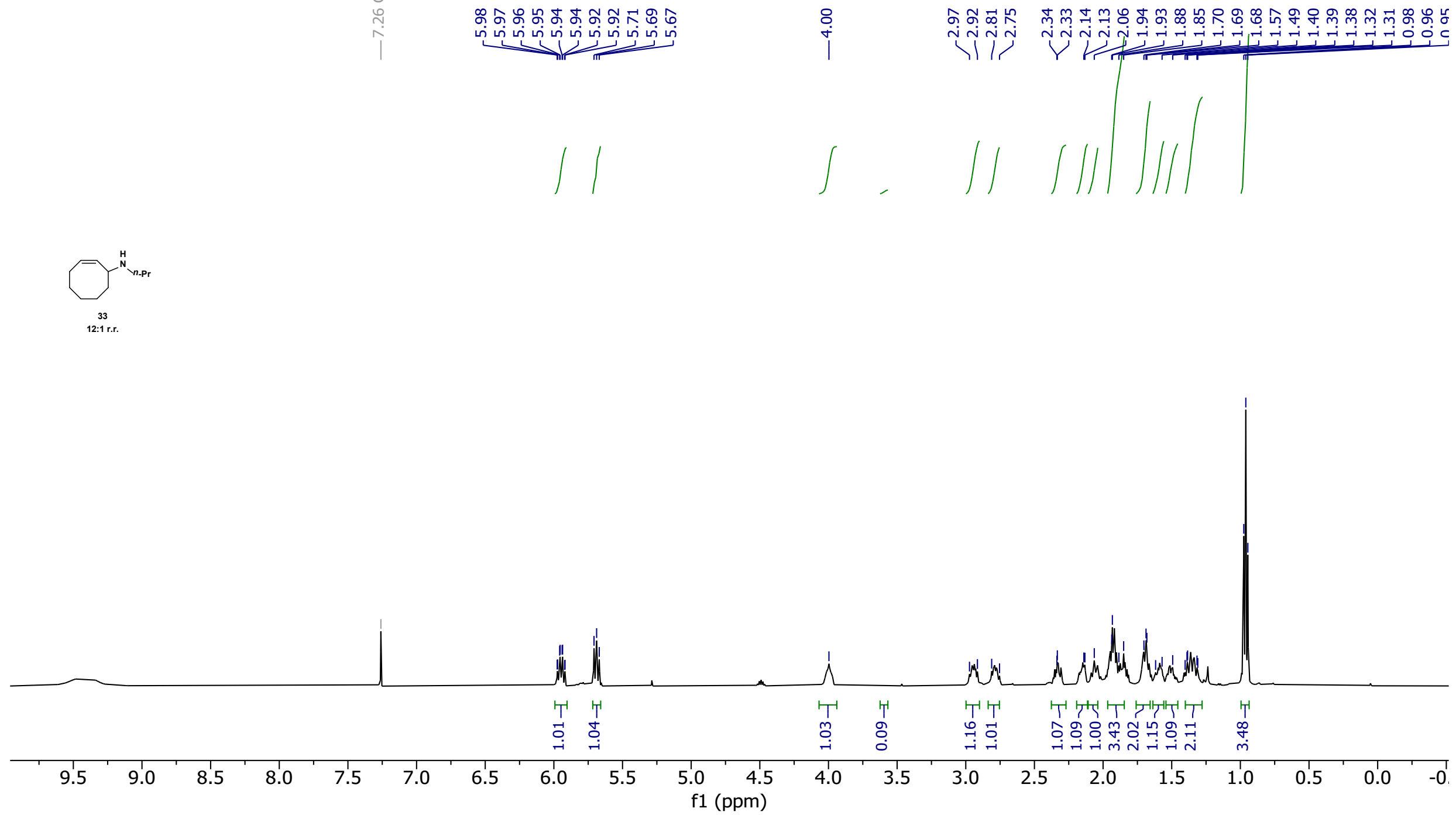
${ }^{13} \mathrm{C}$ NMR of (Z)-N-propyl-2-cyclooctenamine (33)

$\mathrm{CDCl}_{3}, 25^{\circ} \mathrm{C}$

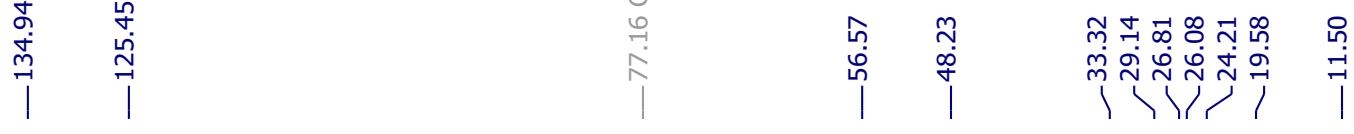

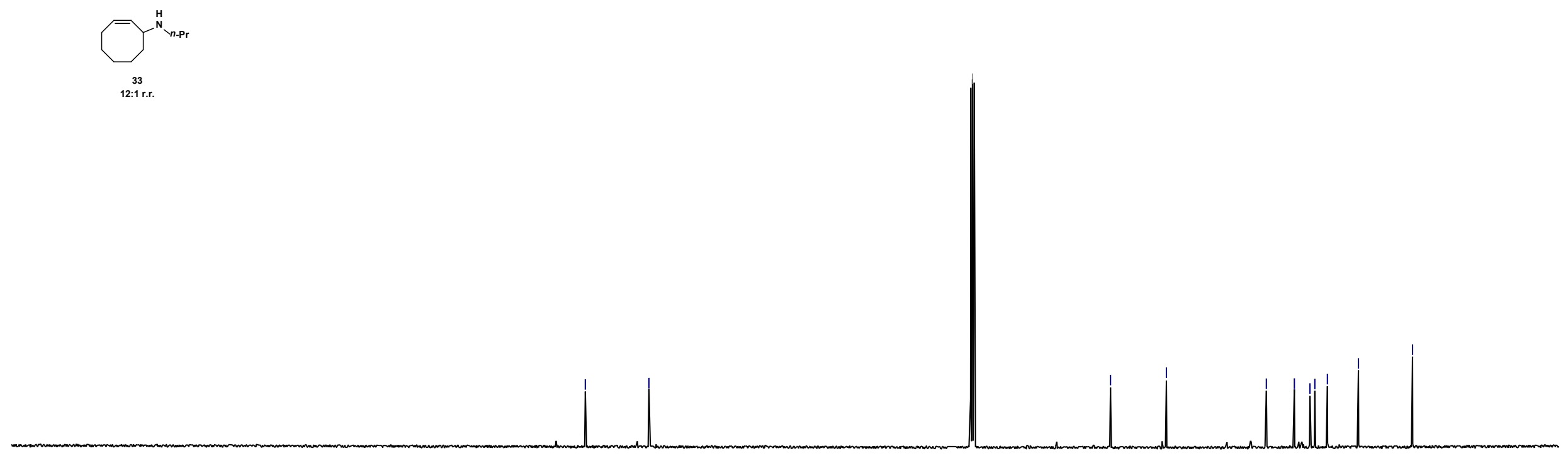

\begin{tabular}{lllllllllllllllllllllllllll}
\hline & 210 & 200 & 190 & 180 & 170 & 160 & 150 & 140 & 130 & 120 & 110 & 100 & 90 & 80 & 70 & 60 & 50 & 40 & 30 & 20 & 10 & 0 & -1
\end{tabular} 
${ }^{1} \mathrm{H}$ NMR of (Z)-N-neopentyl-2-cyclooctenamine (34)

$\mathrm{CDCl}_{3}, 25^{\circ} \mathrm{C}$

剀

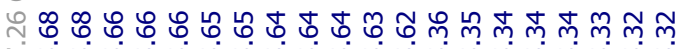

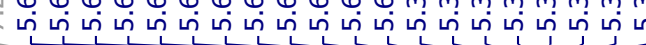

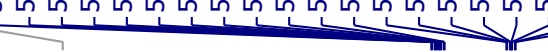

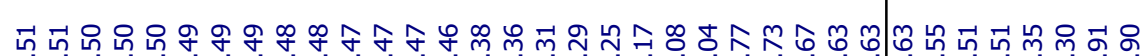

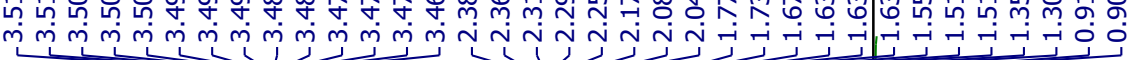
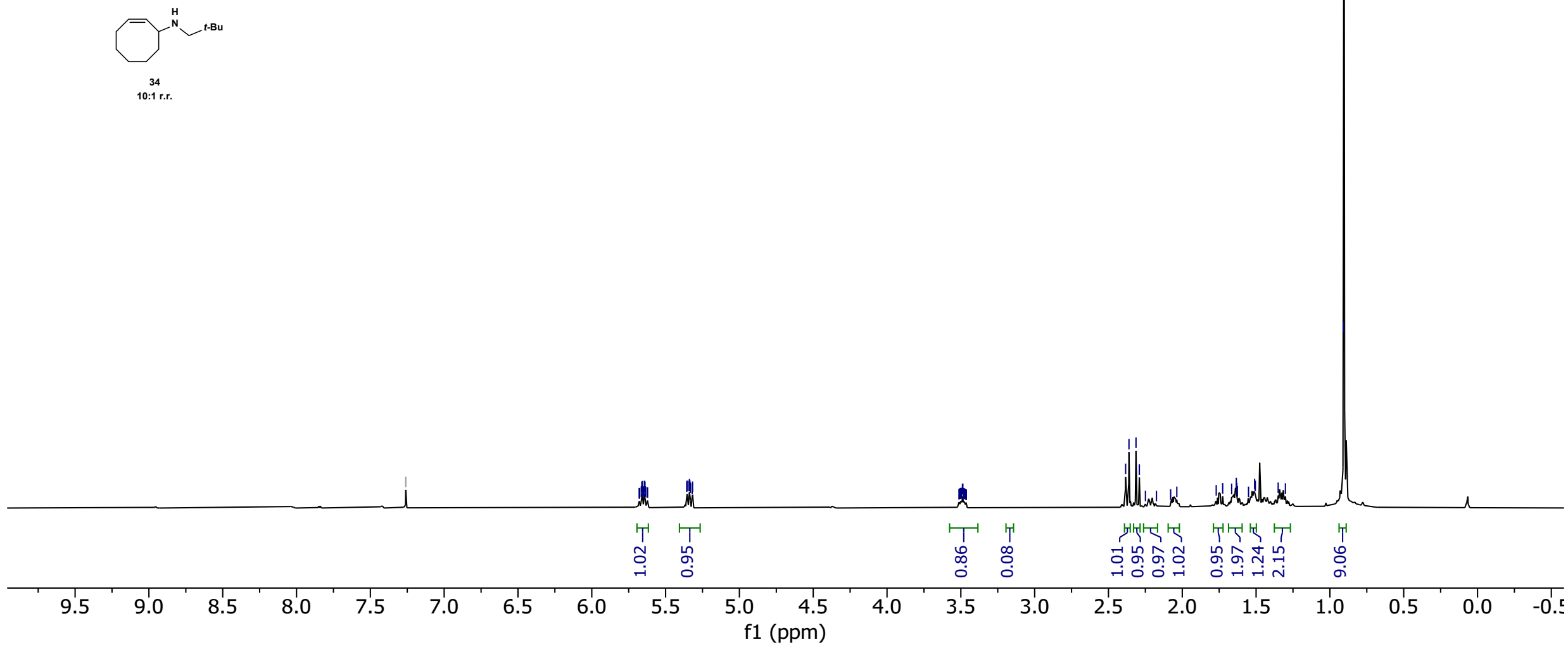
${ }^{13} \mathrm{C}$ NMR of (Z)-N-neopentyl-2-cyclooctenamine (34)

$\mathrm{CDCl}_{3}, 25^{\circ} \mathrm{C}$
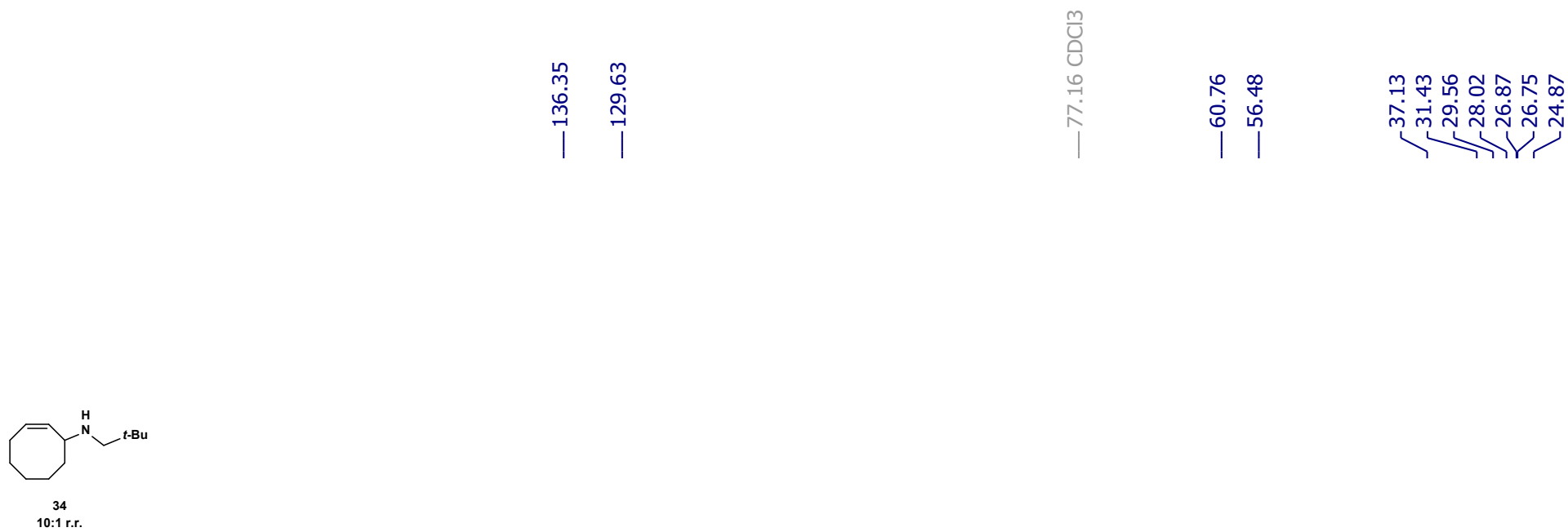

30:1 1 .r.

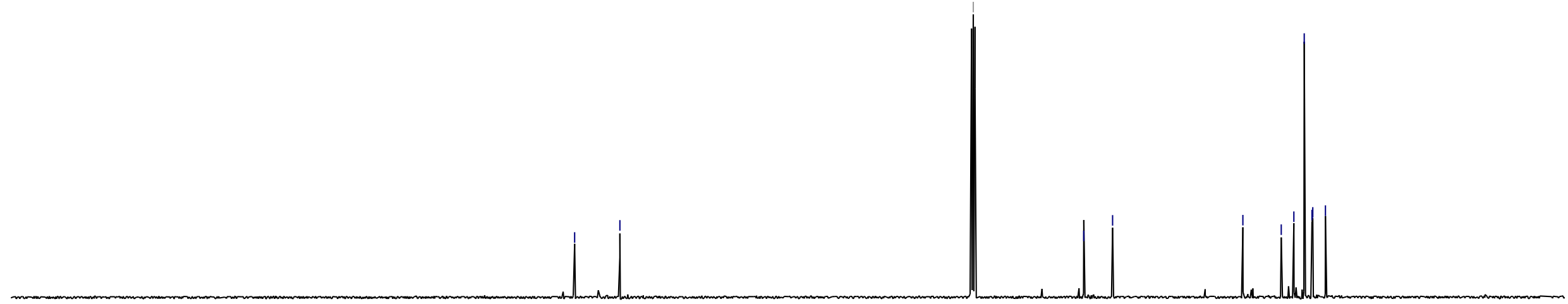

\begin{tabular}{llllllllllllllllllllllllllllllllllll}
\hline 20 & 210 & 200 & 190 & 180 & 170 & 160 & 150 & 140 & 130 & 120 & 110 & 100 & 90 & 80 & 70 & 60 & 50 & 40 & 30 & 20 & 10 & 0 & -1
\end{tabular} 
${ }^{1} \mathrm{H}$ NMR of (Z)-N-adamantyl-2-cyclooctenamine (35)

$\mathrm{CDCl}_{3}, 25^{\circ} \mathrm{C}$

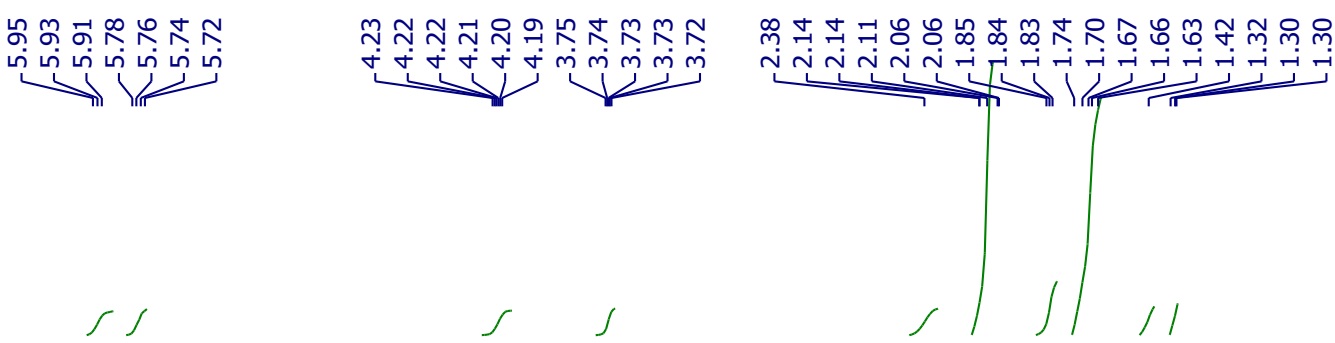

$\sum_{\substack{350 \\ 20: 11 . r .}}^{H}{ }^{H}$

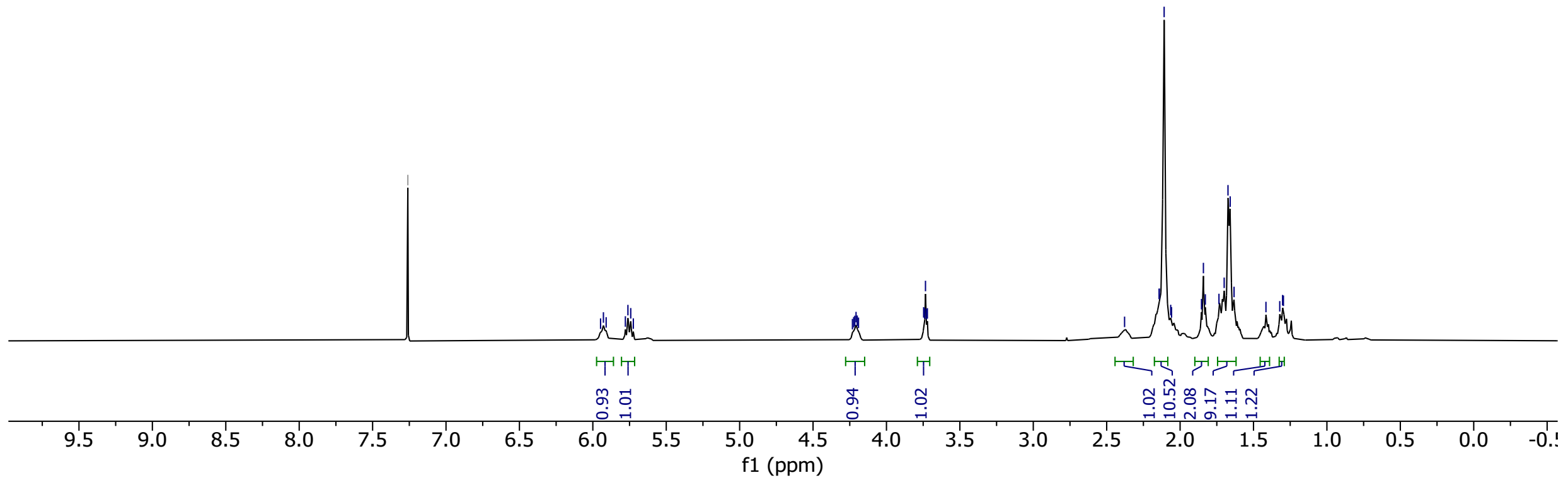


${ }^{13} \mathrm{C}$ NMR of (Z)-N-adamantyl-2-cyclooctenamine (35)

$\mathrm{CDCl}_{3}, 25^{\circ} \mathrm{C}$

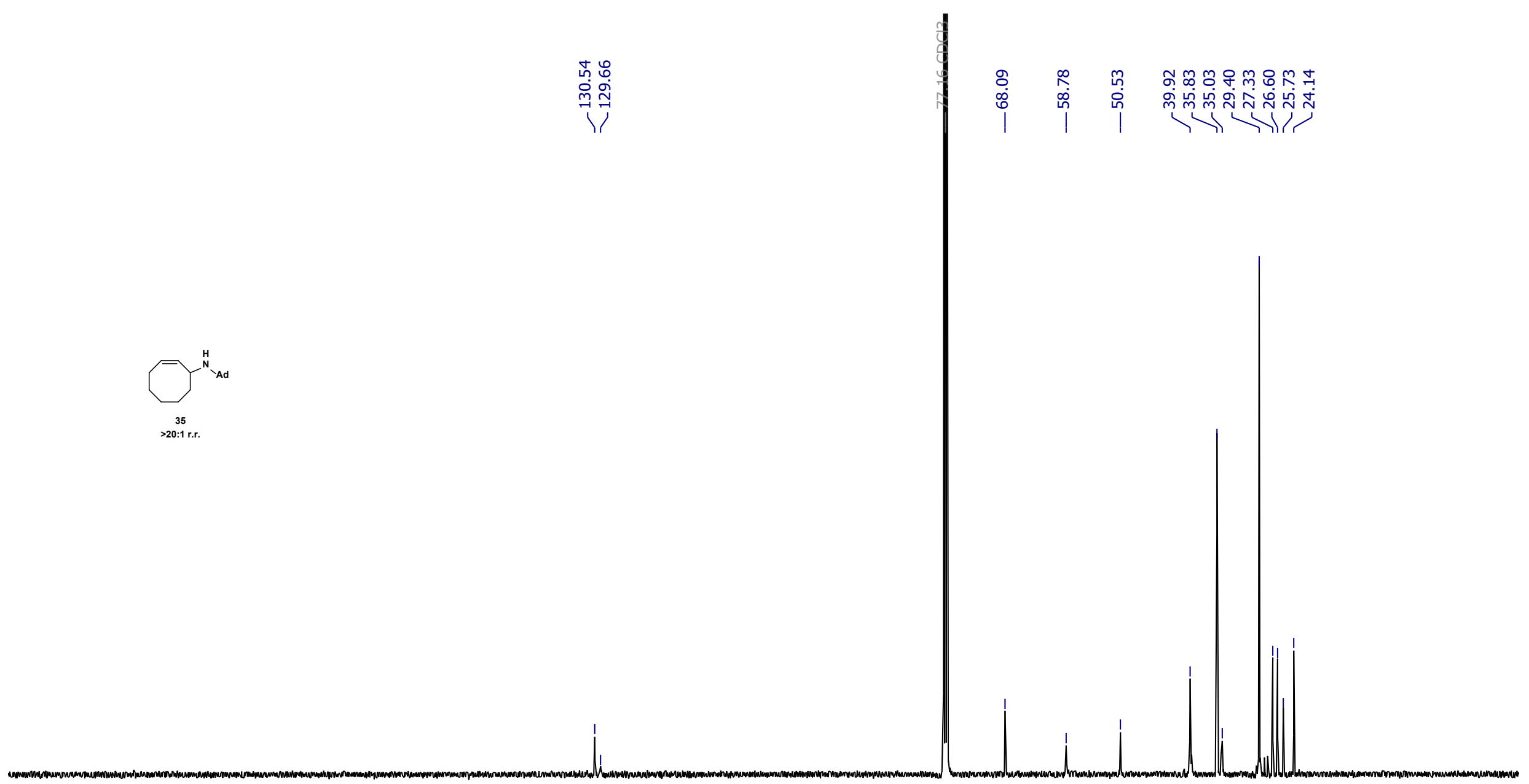

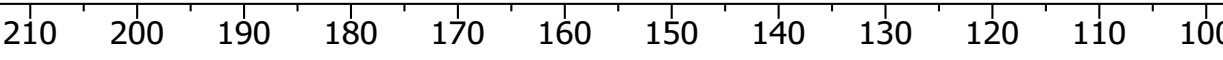
f1 (ppm) 
${ }^{1} \mathrm{H}$ NMR of (Z)-2-(2-cyclooctenylamino)ethyl pivalate (36)

$\mathrm{CDCl}_{3}, 25^{\circ} \mathrm{C}$

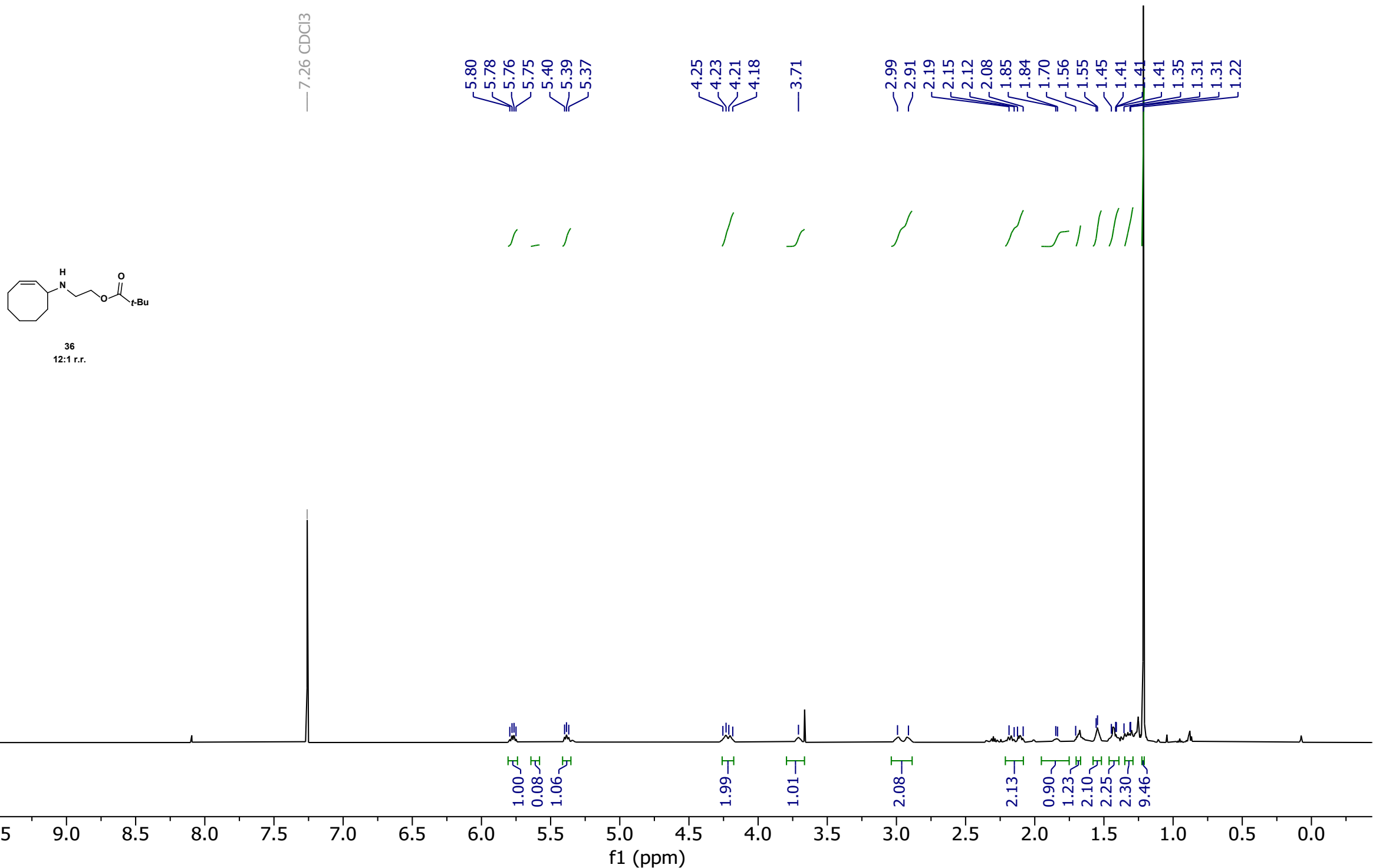


${ }^{13} \mathrm{C}$ NMR of (Z)-2-(2-cyclooctenylamino)ethyl pivalate (36)

$\mathrm{CDCl}_{3}, 25^{\circ} \mathrm{C}$

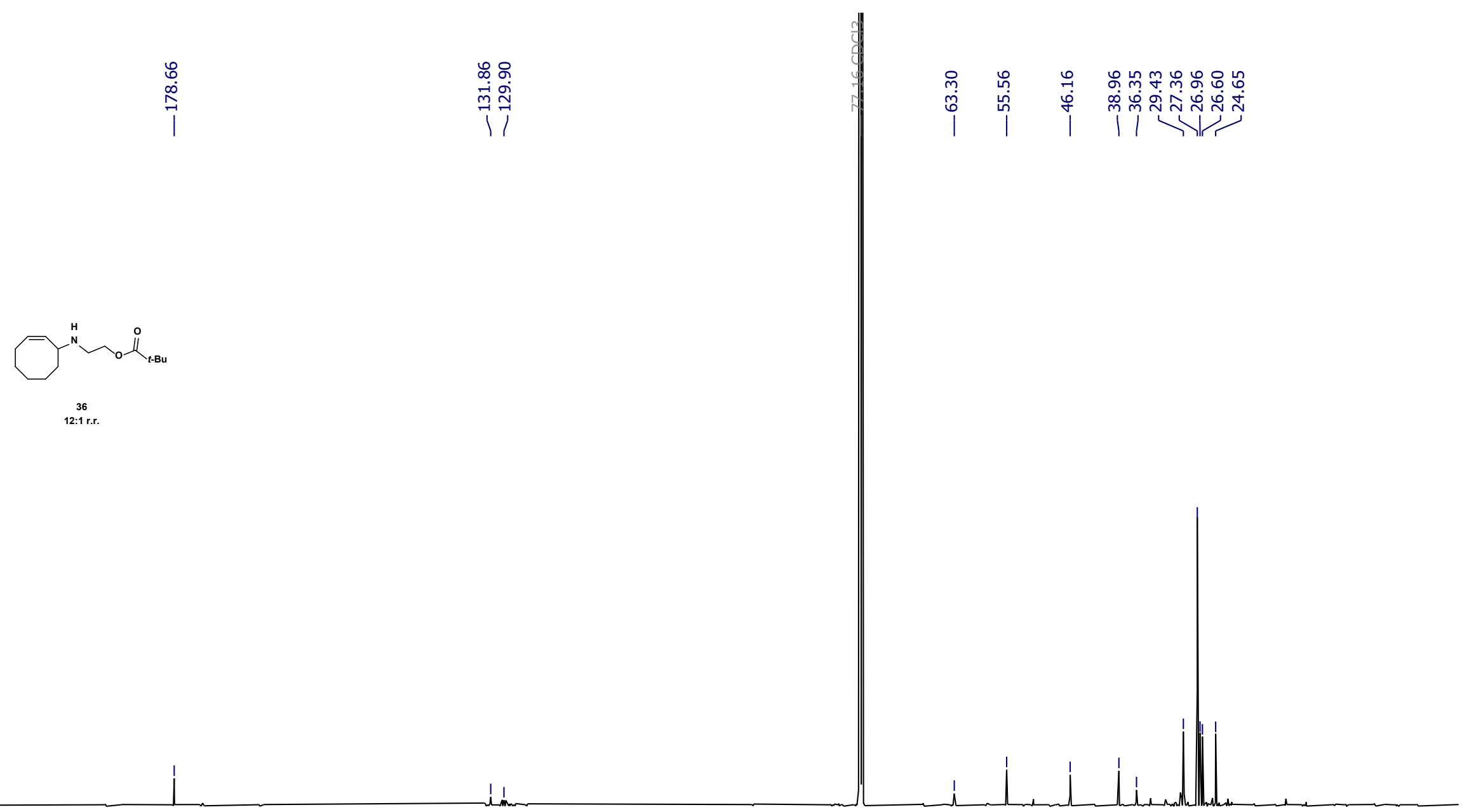

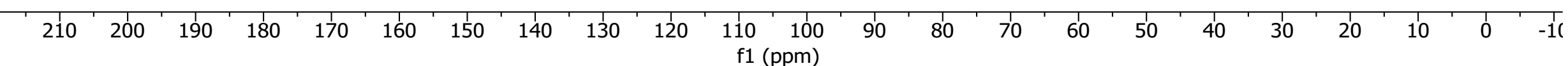


${ }^{1} \mathrm{H}$ NMR of (Z)-3-(cyclooct-2-en-1-ylamino)propan-1-ol (37)

$\mathrm{CDCl}_{3}, 25^{\circ} \mathrm{C}$

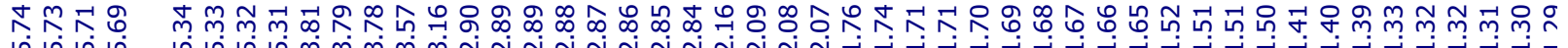

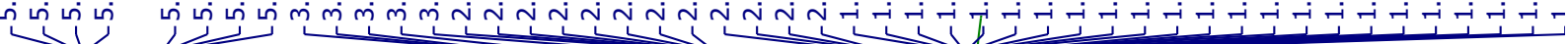

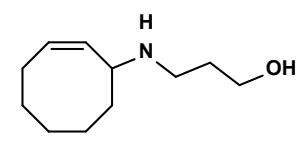

37
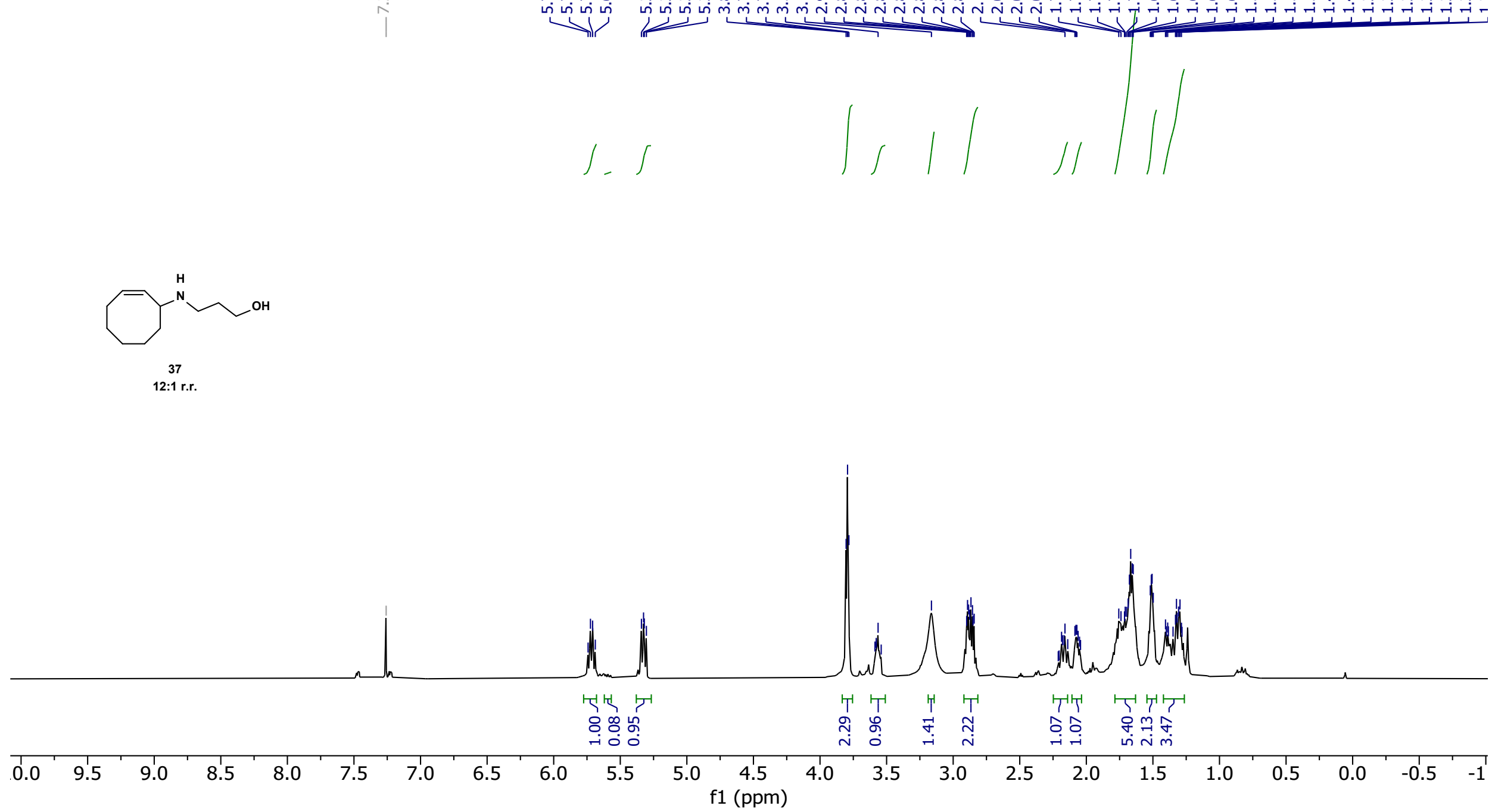
${ }^{13}$ C NMR of (Z)-3-(cyclooct-2-en-1-ylamino)propan-1-ol (37)

$\mathrm{CDCl}_{3}, 25^{\circ} \mathrm{C}$



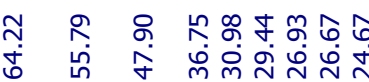

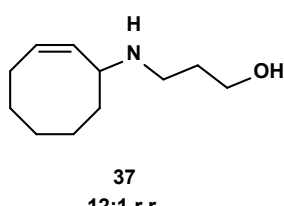

$12: 1$

\begin{tabular}{lllllllllllllllllllllllllllllllllllllll}
\hline 250 & 240 & 230 & 220 & 210 & 200 & 190 & 180 & 170 & 160 & 150 & 140 & 130 & 120 & 110 & 100 & 90 & 80 & 70 & 60 & 50 & 40 & 30 & 20 & 10 & 0 & -10 & -20 & -30 & -40 & -50
\end{tabular} f1 (ppm) 
${ }^{1} \mathrm{H}$ NMR of ethyl (E)-2-methyl-2-(4-phenyl-2-butenylamino)propanoate (38)

$\mathrm{CDCl}_{3}, 25^{\circ} \mathrm{C}$

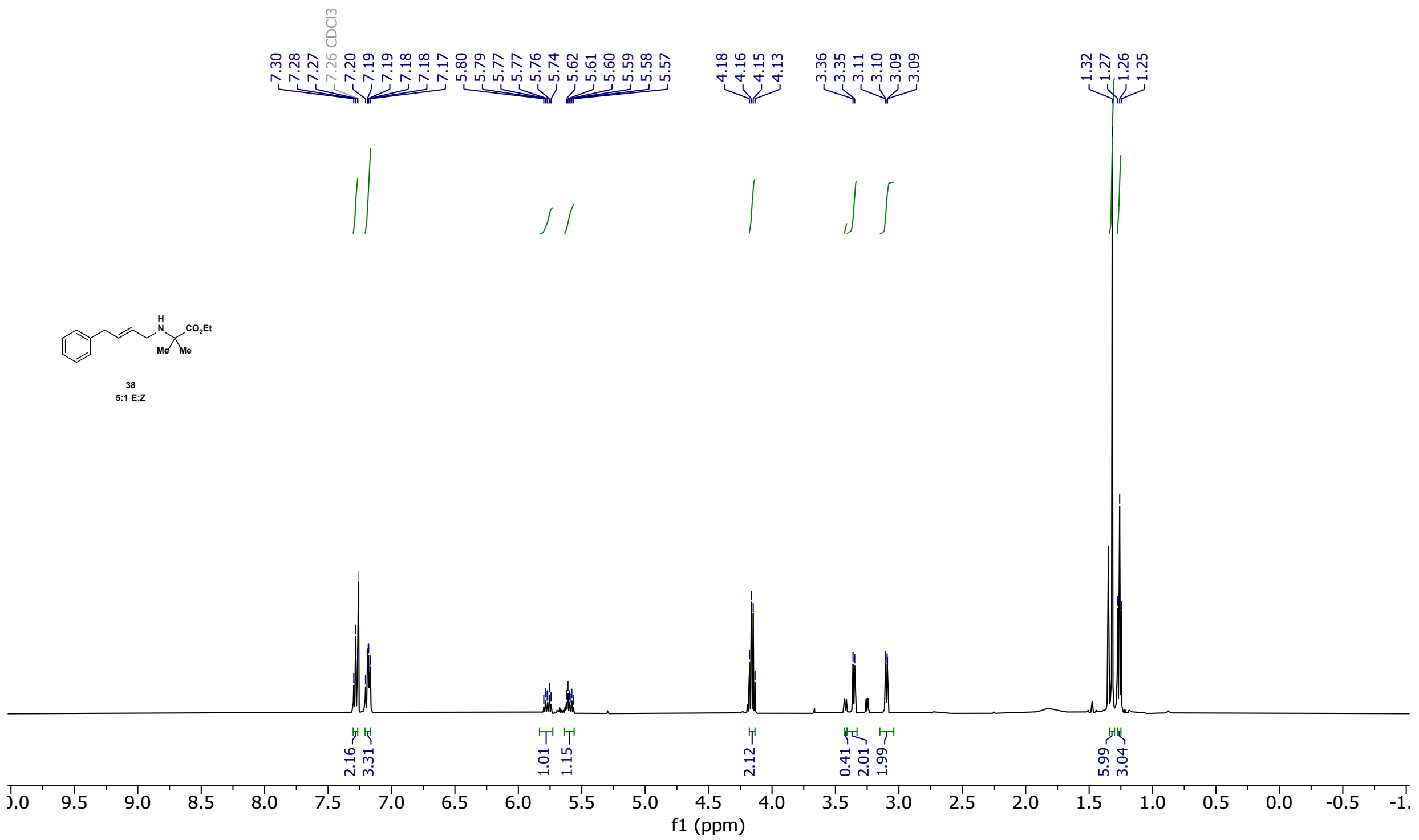


${ }^{13}$ C NMR of ethyl (E)-2-methyl-2-(4-phenyl-2-butenylamino)propanoate (38)

$\mathrm{CDCl}_{3}, 25^{\circ} \mathrm{C}$

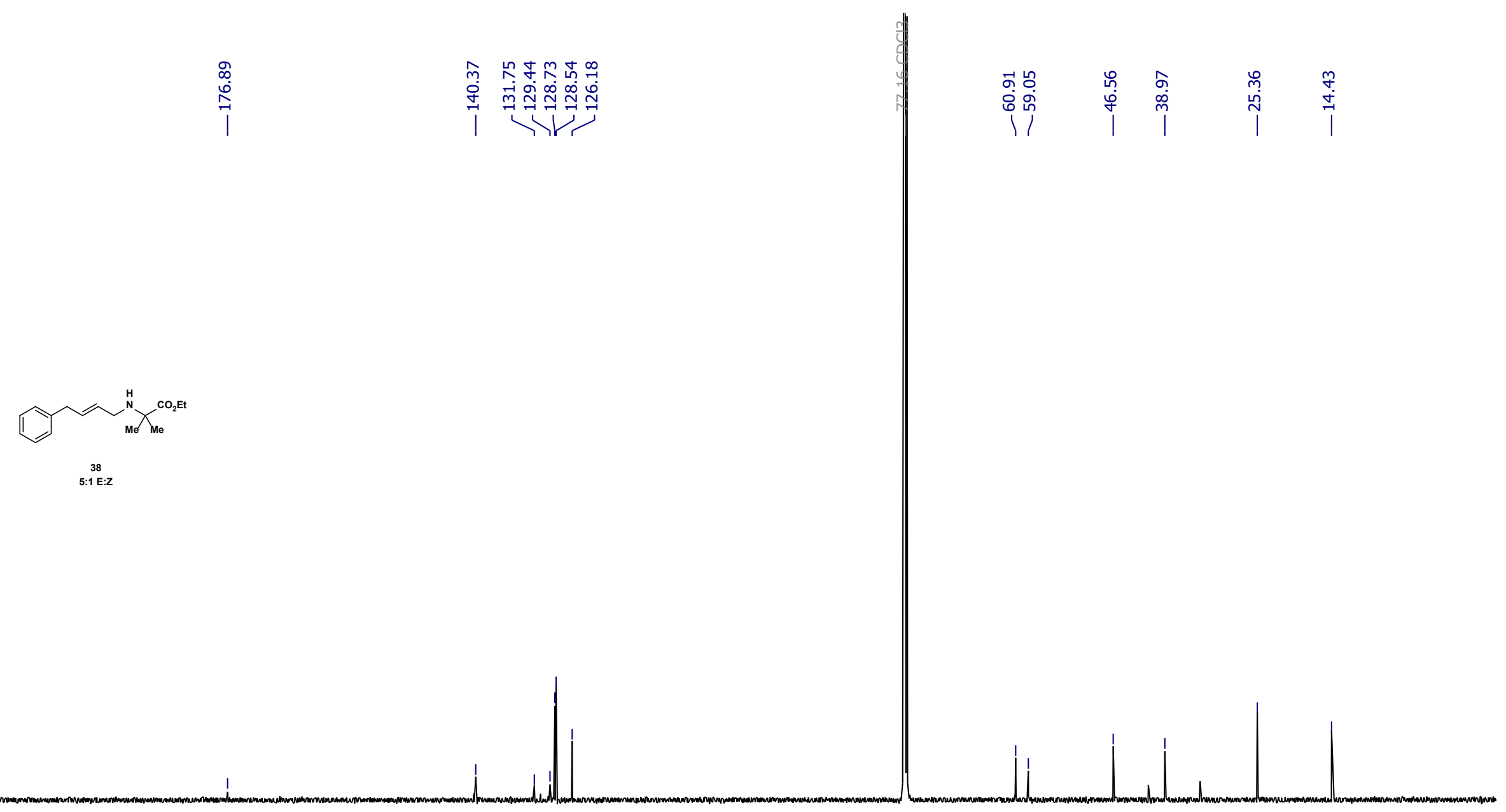

\begin{tabular}{llllllllllllllllllllllllll}
\hline & 210 & 200 & 190 & 180 & 170 & 160 & 150 & 140 & 130 & 120 & $\begin{array}{c}110 \\
\mathrm{f} 1(\mathrm{ppm})\end{array}$ & 100 & 90 & 80 & 70 & 60 & 50 & 40 & 30 & 20 & 10 & 0 & 1
\end{tabular} 
${ }^{1} \mathrm{H}$ NMR of $(E)-5$-methyl-N-(1-phenylethyl)-2-hexenamine (39)

$\mathrm{CDCl}_{3}, 25^{\circ} \mathrm{C}$

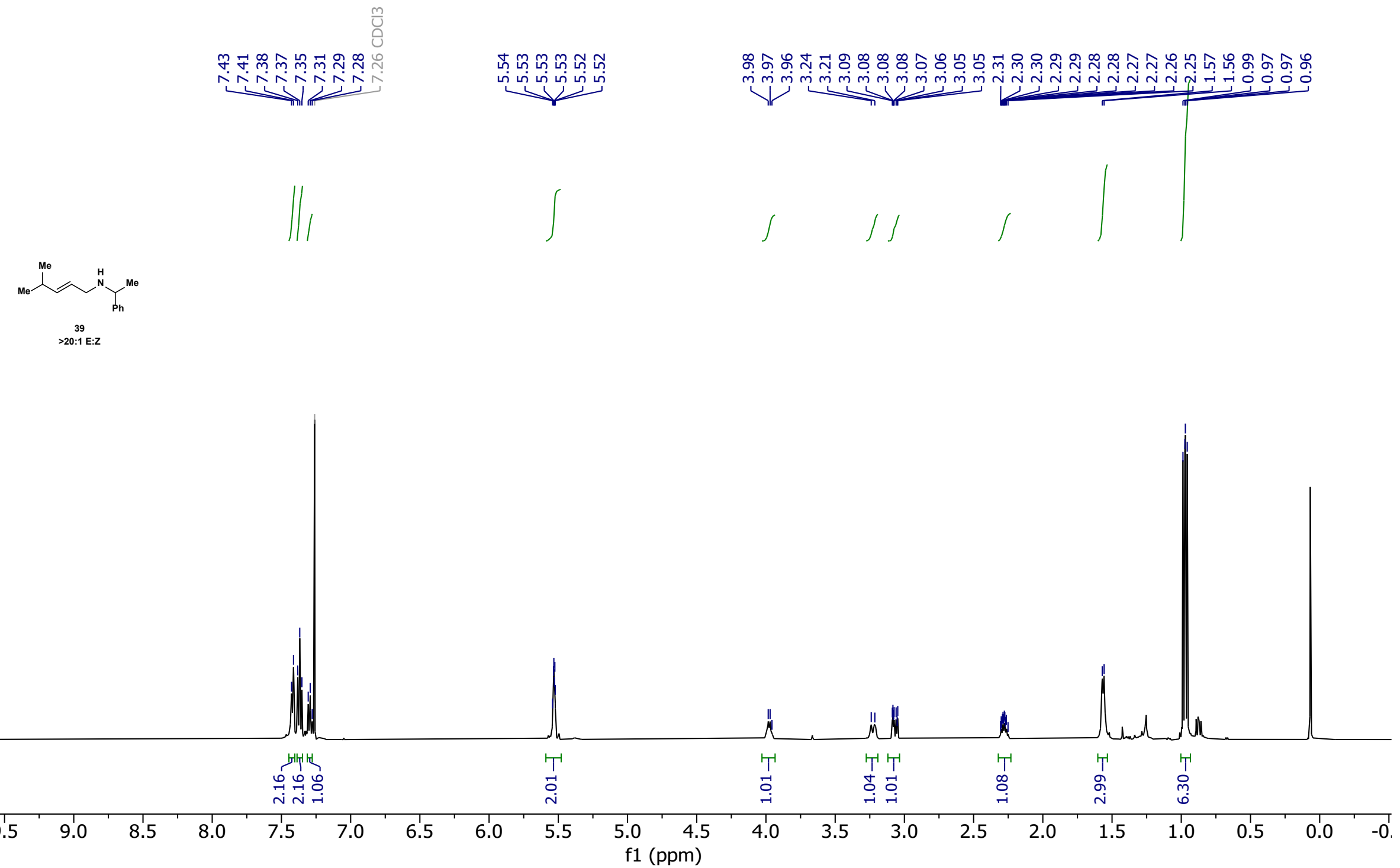


${ }^{13} \mathrm{C}$ NMR of (E)-5-methyl-N-(1-phenylethyl)-2-hexenamine (39)

$\mathrm{CDCl}_{3}, 25^{\circ} \mathrm{C}$

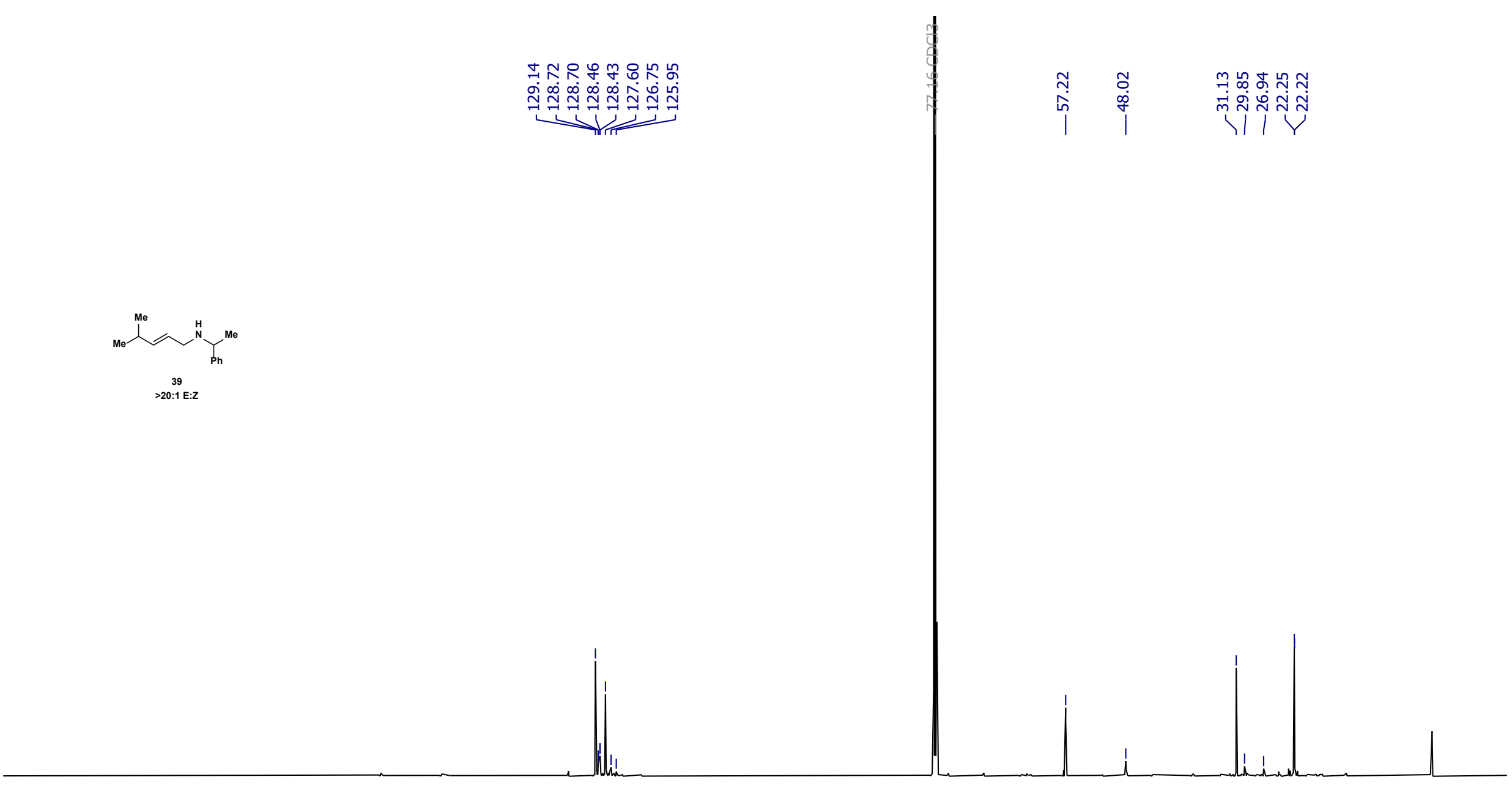

$\begin{array}{llllllllllllllllllllllllll}210 & 200 & 190 & 180 & 170 & 160 & 150 & 140 & 130 & 120 & \begin{array}{c}110 \\ \mathrm{f} 1(\mathrm{ppm})\end{array} & 90 & 80 & 70 & 60 & 50 & 40 & 30 & 20 & 10 & 0 & -1\end{array}$


${ }^{1} \mathrm{H}$ NMR of $\mathrm{N}$-(tert-butyl)-3-phenyl-2-((2,2,6,6-tetramethylpiperidin-1-yl)oxy)propanamine (40)

$\mathrm{CDCl}_{3}, 25^{\circ} \mathrm{C}$

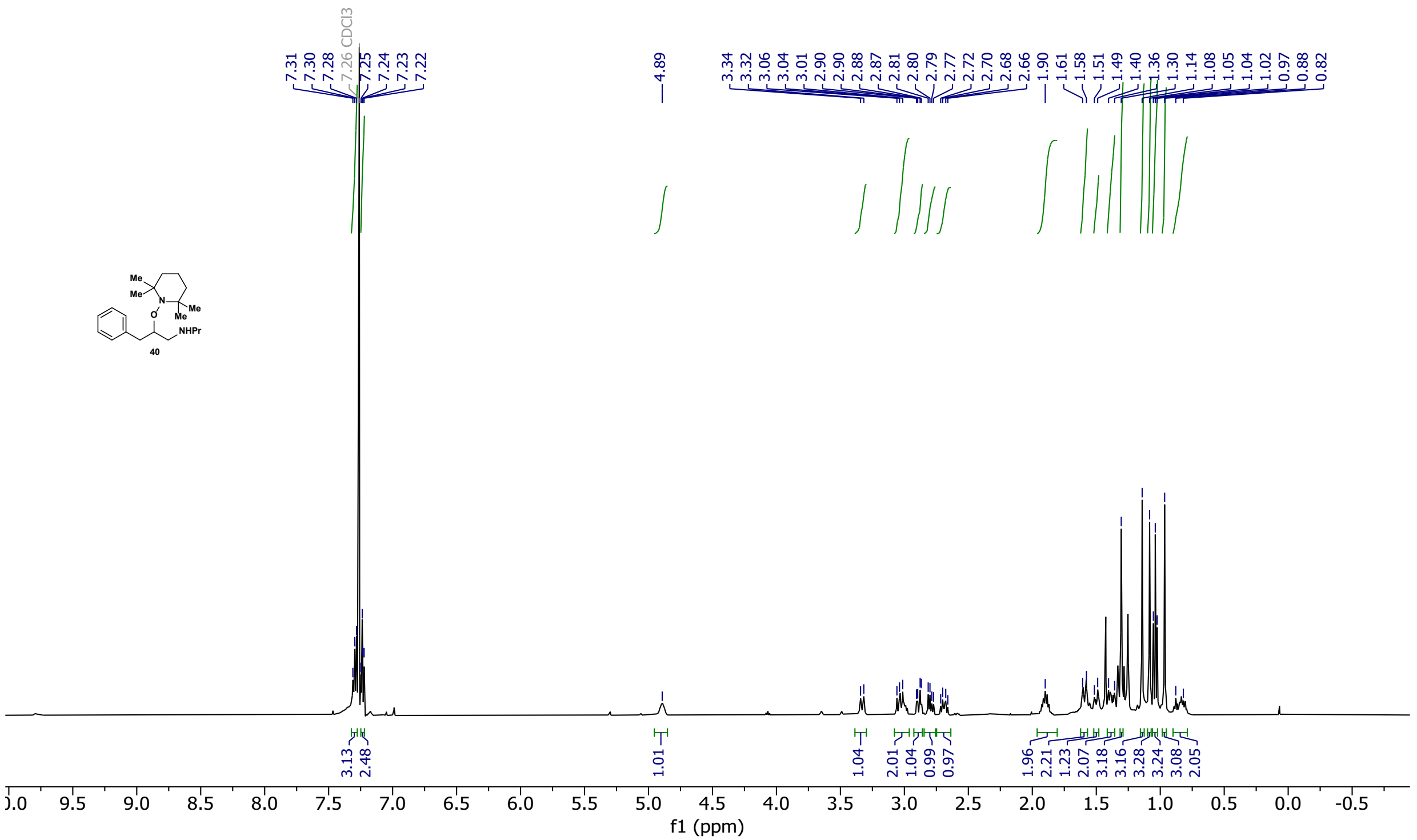


${ }^{13}$ C NMR of $N$-(tert-butyl)-3-phenyl-2-((2,2,6,6-tetramethylpiperidin-1-yl)oxy)propanamine (40)

$\mathrm{CDCl}_{3}, 25^{\circ} \mathrm{C}$

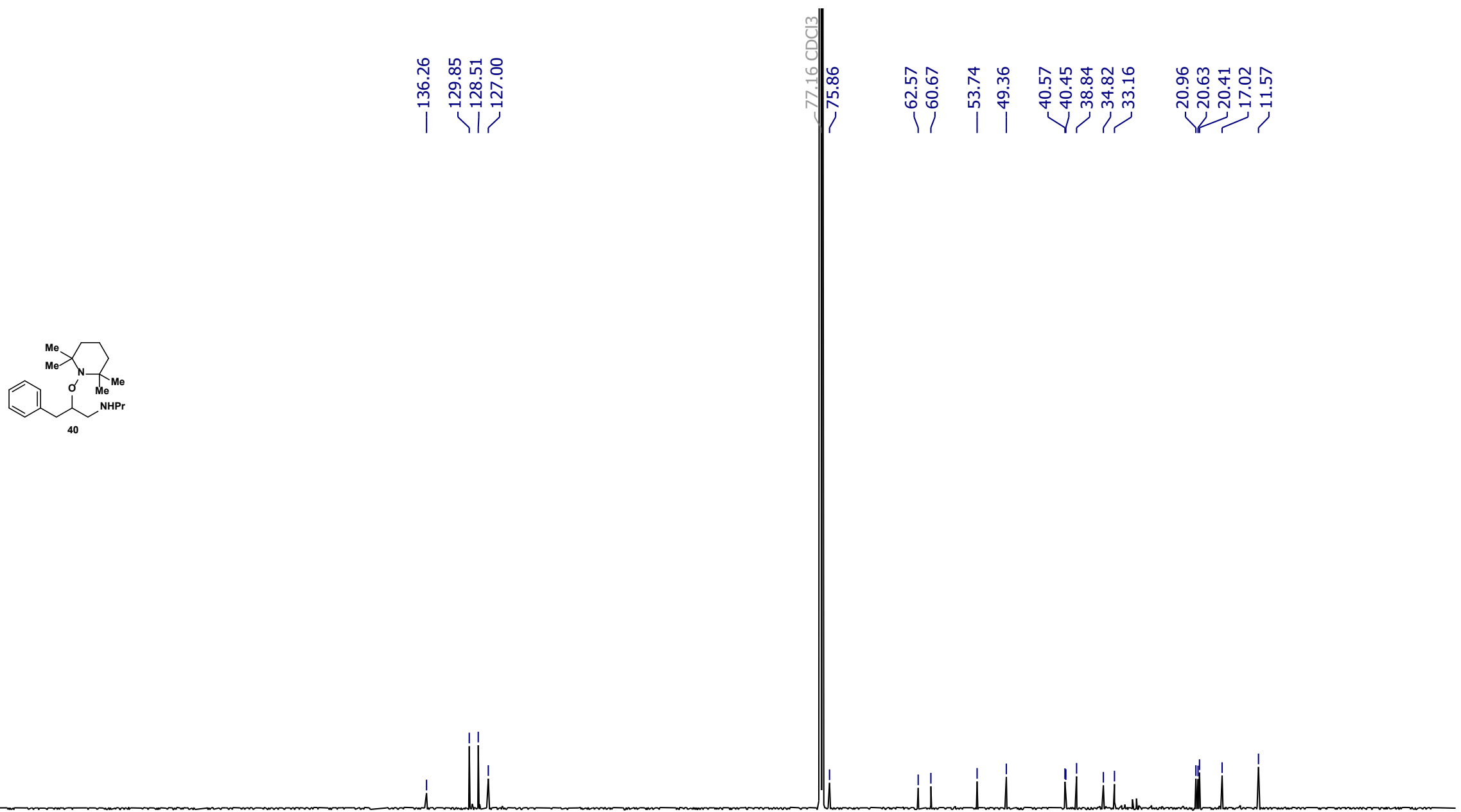

$\begin{array}{llllllllllllllllllllllll}210 & 200 & 190 & 180 & 170 & 160 & 150 & 140 & 130 & 120 & 110 & 100 & 90 & 80 & 70 & 60 & 50 & 40 & 30 & 20 & 10 & 0 & -10\end{array}$


${ }^{1} \mathrm{H}$ NMR of (E)-N-(5,5-dichloropenta-2,4-dien-1-yl)cyclohexanamine (41)

$\mathrm{CDCl}_{3}, 25^{\circ} \mathrm{C}$

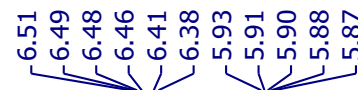

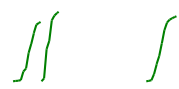

户் $\sqrt{1} \underbrace{N}$
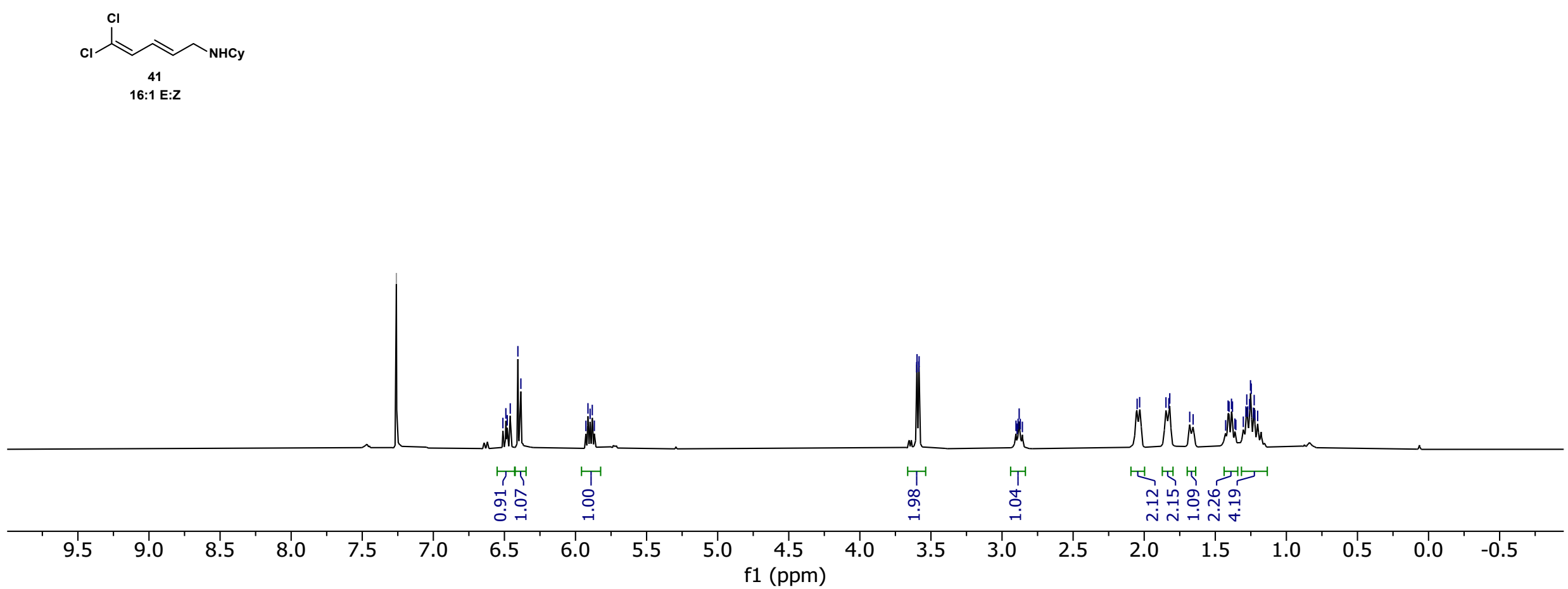


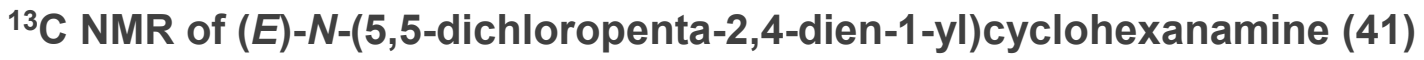

$\mathrm{CDCl}_{3}, 25{ }^{\circ} \mathrm{C}$

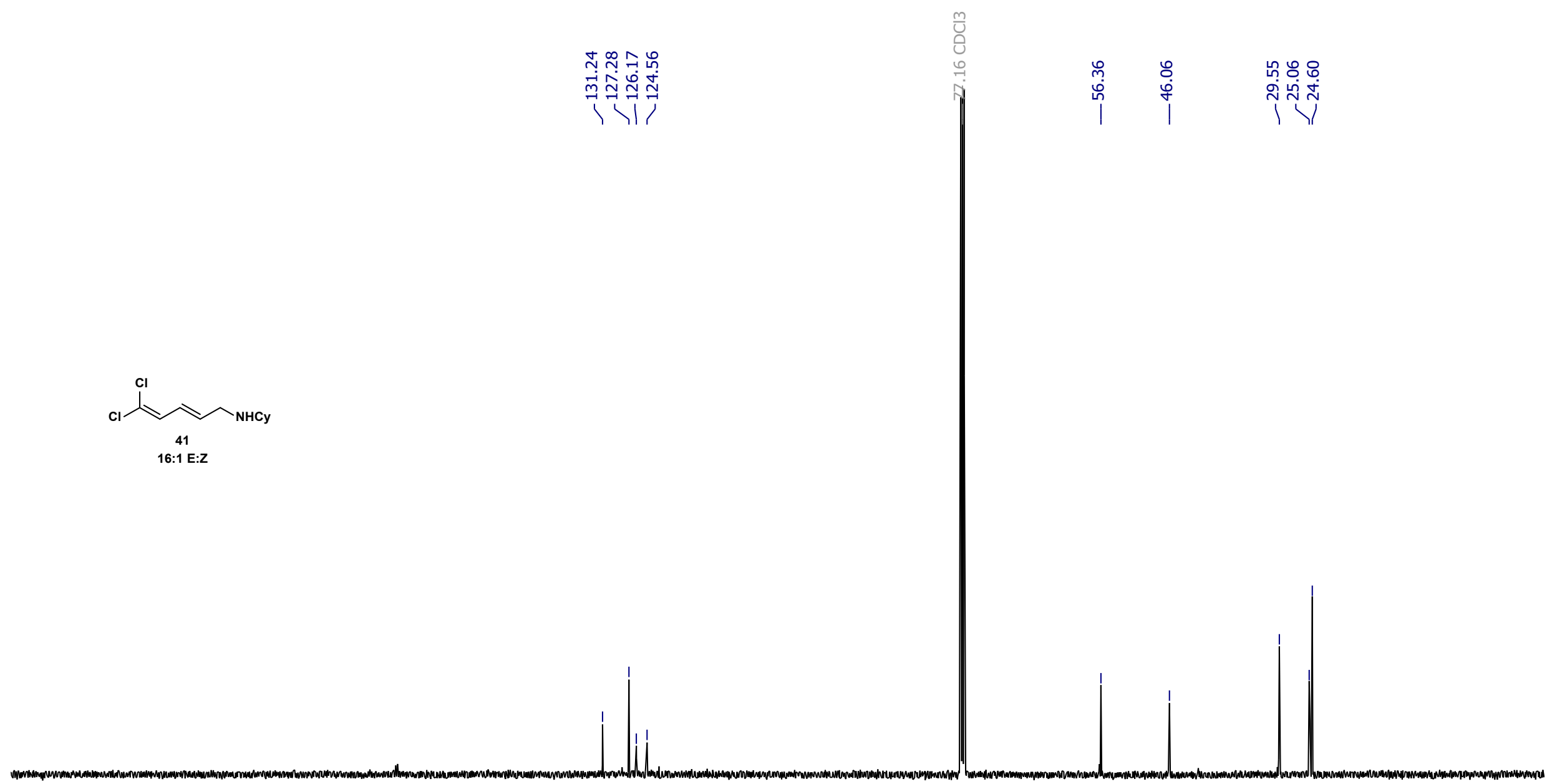

$\begin{array}{lllllllllllllllllllllllll}20 & 210 & 200 & 190 & 180 & 170 & 160 & 150 & 140 & 130 & 120 & \begin{array}{c}110 \\ \mathrm{f} 1(\mathrm{ppm})\end{array} & 100 & 80 & 70 & 60 & 50 & 40 & 30 & 20 & 10 & 0 & -1\end{array}$


${ }^{1}$ H NMR of 2-(2-methylprop-1-en-1-yl)hex-5-en-1-yl acetate (42)

$\mathrm{CDCl}_{3}, 25^{\circ} \mathrm{C}$
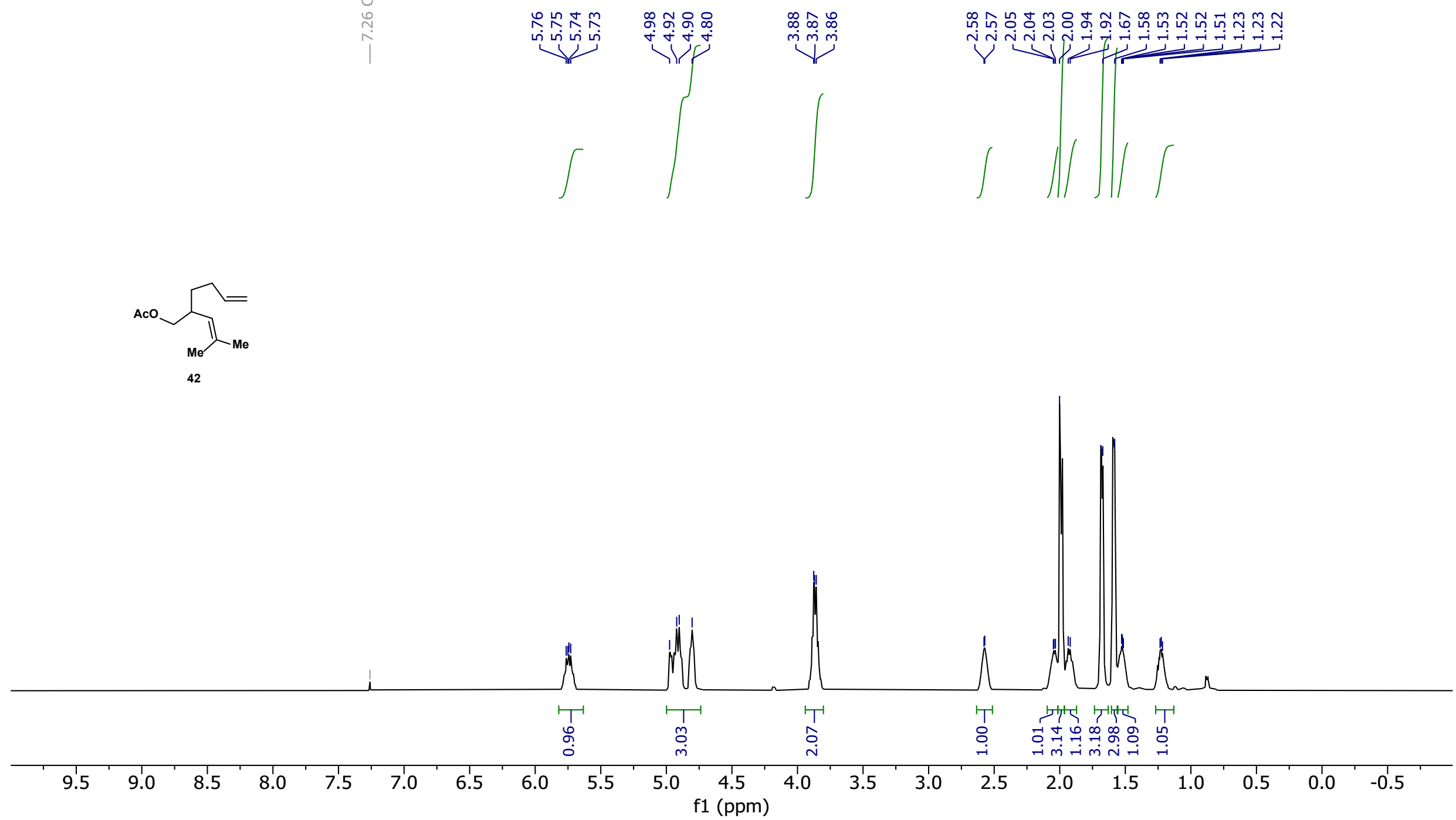
${ }^{13} \mathrm{C}$ NMR of 2-(2-methylprop-1-en-1-yl)hex-5-en-1-yl acetate (42)

$\mathrm{CDCl}_{3}, 25^{\circ} \mathrm{C}$

م

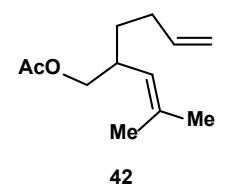

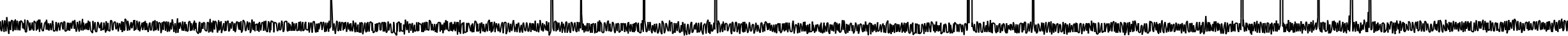

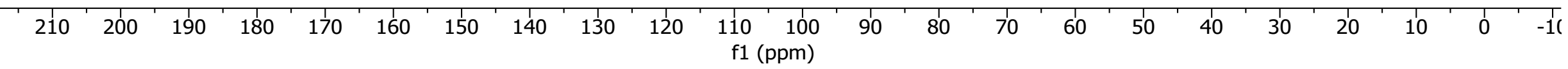


${ }^{1} \mathrm{H}$ NMR of (3-((cyclohexylamino)methyl)-2-(prop-1-en-2-yl)cyclopentyl)methyl acetate (43)

$\mathrm{CDCl}_{3}, 25^{\circ} \mathrm{C}$
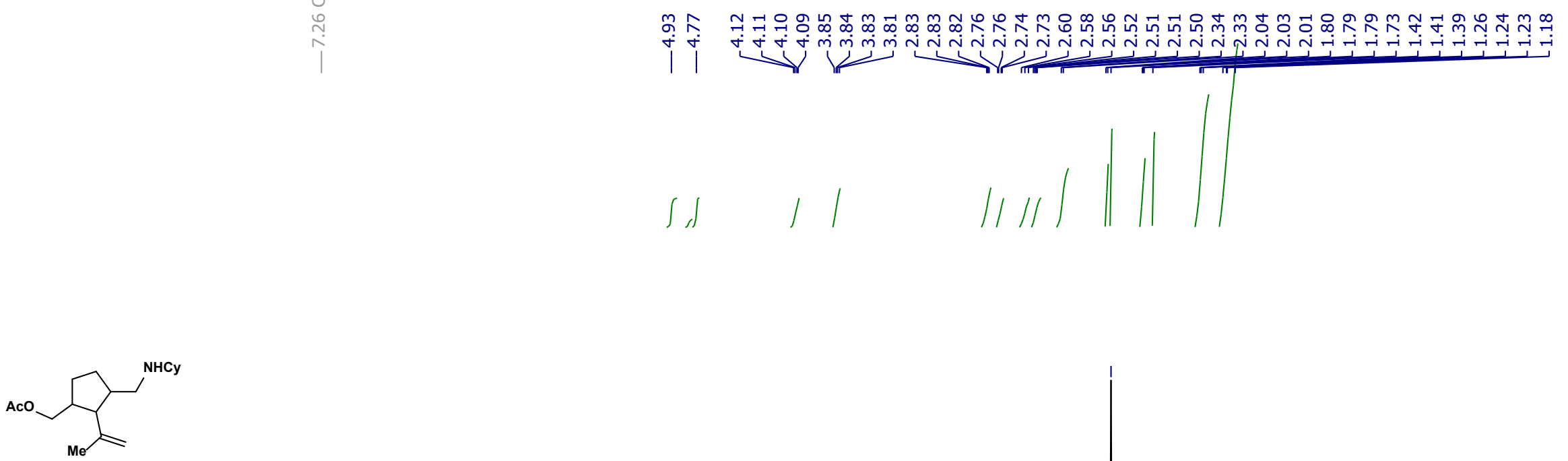

4:1

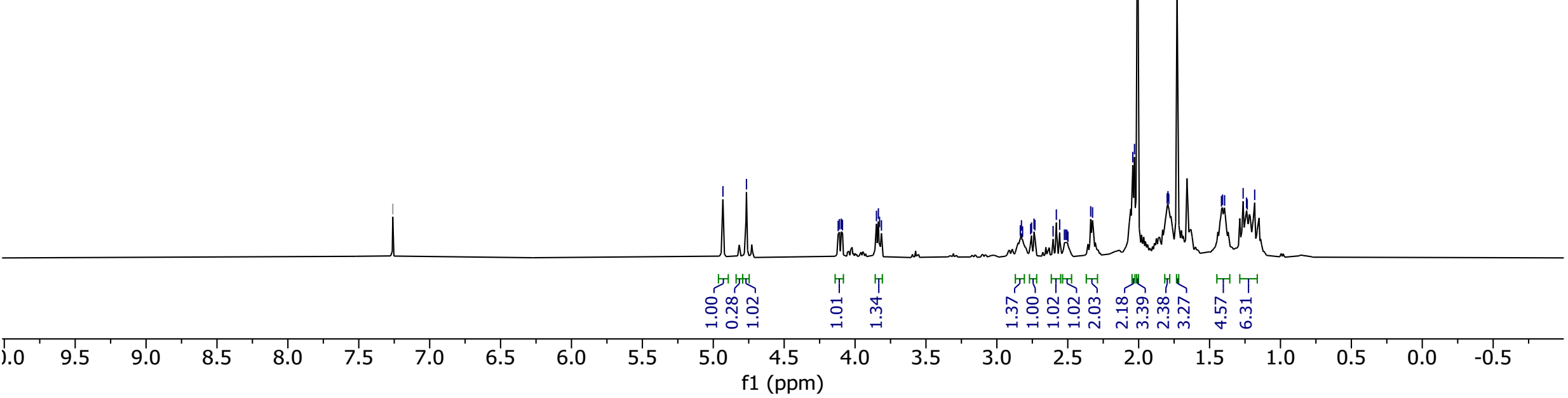


${ }^{13} \mathrm{C}$ NMR of (3-((cyclohexylamino)methyl)-2-(prop-1-en-2-yl)cyclopentyl)methyl acetate (43)

$\mathrm{CDCl}_{3}, 25^{\circ} \mathrm{C}$

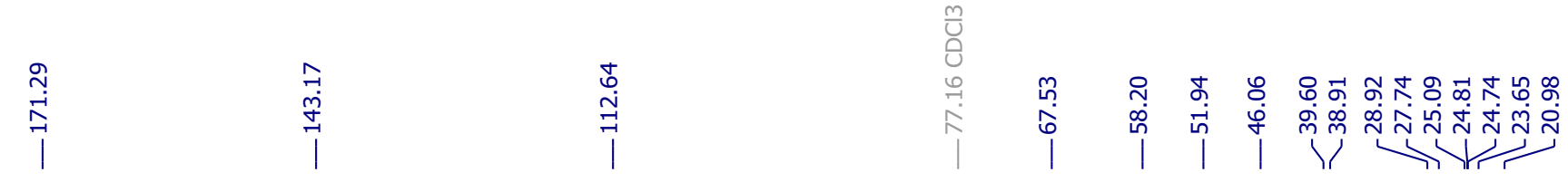

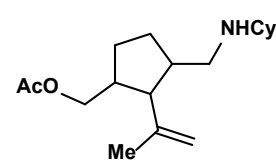

43
$3: 1 \mathrm{dr}$

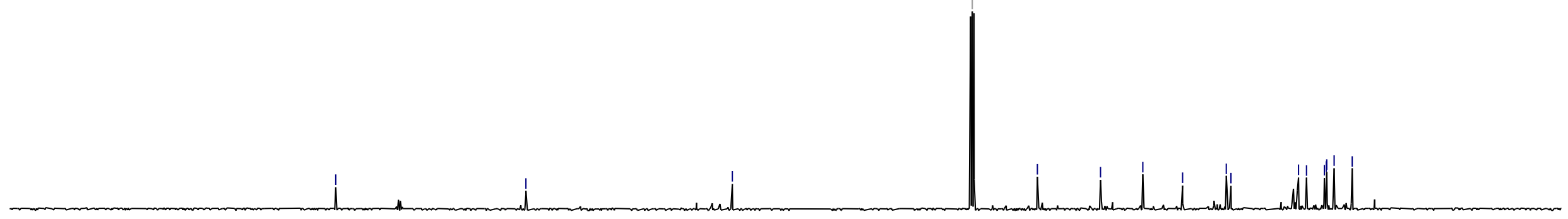

210

$200 \quad 1$

$\begin{array}{llll}90 & 180 & 170 & 160\end{array}$

$150 \quad 140$

130

120110 10100

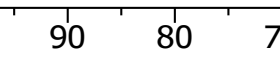

$60 \quad 50$ 
${ }^{1} \mathrm{H}$ NMR of $\mathrm{N}$-cyclohexyl-5H-dibenzo[b,d]thiophen-5-imine (44)

$\mathrm{CDCl}_{3}, 25^{\circ} \mathrm{C}$
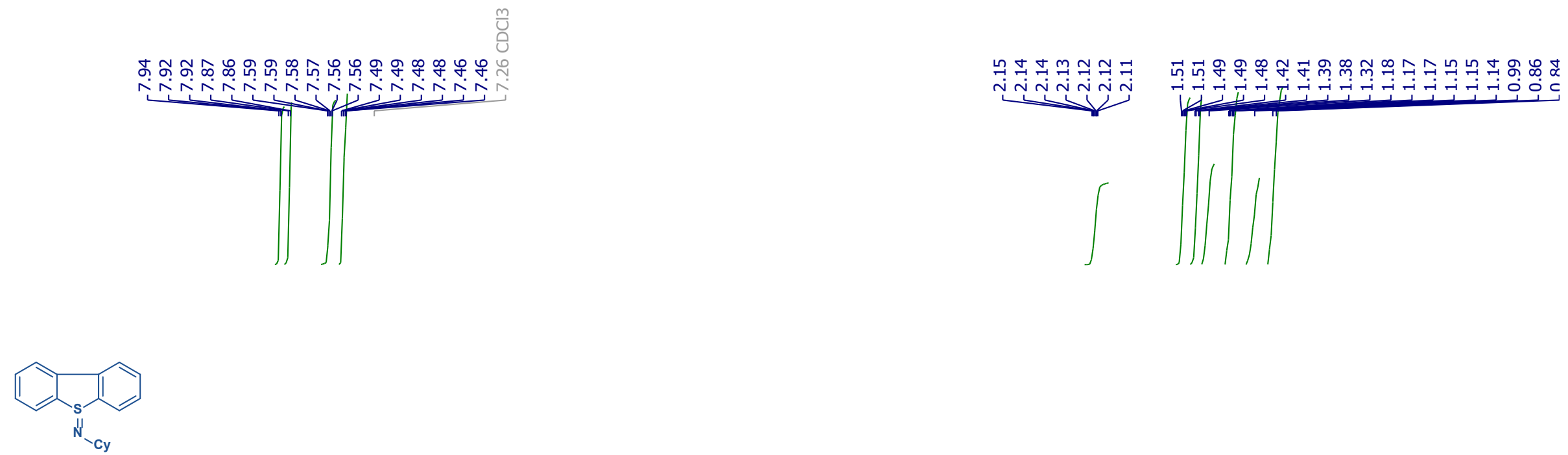

44

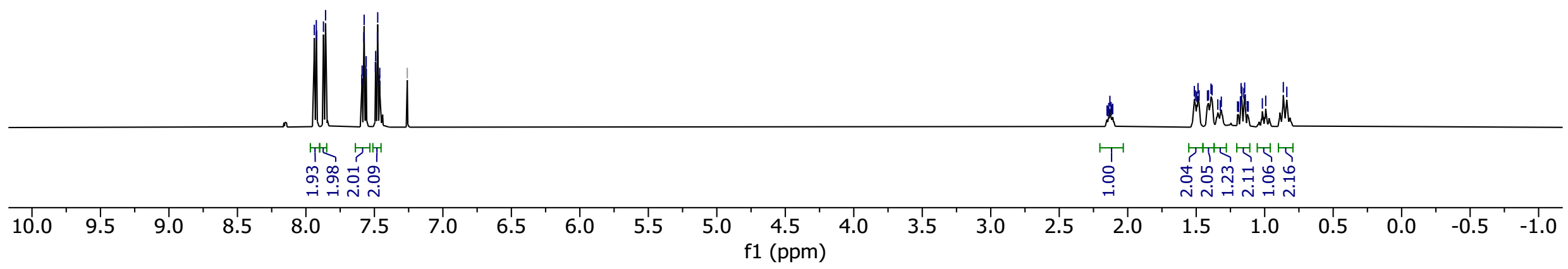


${ }^{13} \mathrm{C}$ NMR of $\mathrm{N}$-cyclohexyl-5H-dibenzo[b,d]thiophen-5-imine (44)

$\mathrm{CDCl}_{3}, 25{ }^{\circ} \mathrm{C}$

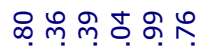

于ें

1
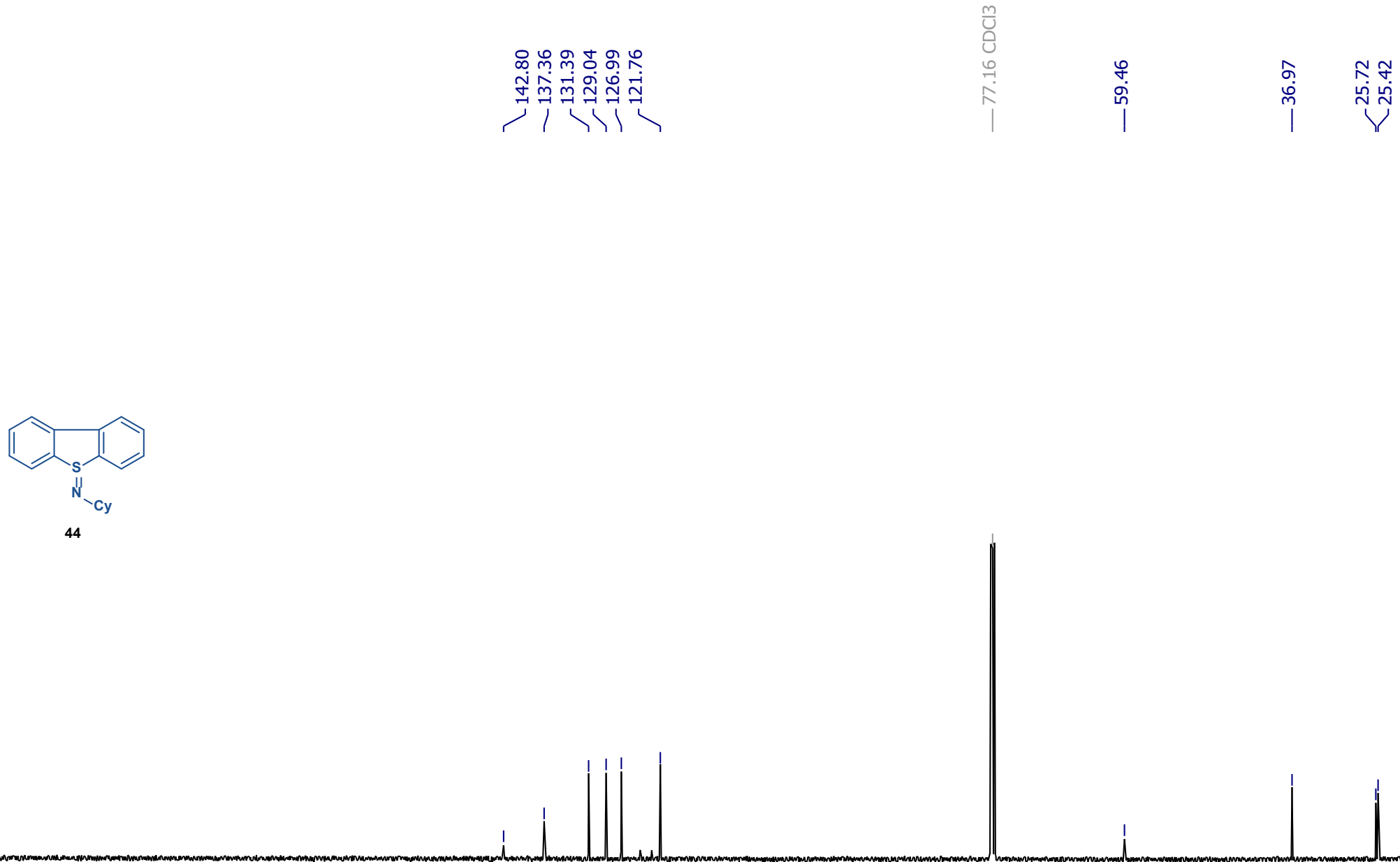

$\begin{array}{lllllllllllllllllllllllllll}20 & 210 & 200 & 190 & 180 & 170 & 160 & 150 & 140 & 130 & 120 & \begin{array}{c}1 \\ \mathrm{f} 1\end{array}(\mathrm{ppm}) & 100 & 90 & 80 & 70 & 60 & 50 & 40 & 30 & 20 & 10 & 0 & -1\end{array}$ 
${ }^{1} \mathrm{H}$ NMR of 1,1-bis(4-chlorophenyl)-N-cyclohexylsulfanimine (45)

$\mathrm{CDCl}_{3}, 25^{\circ} \mathrm{C}$

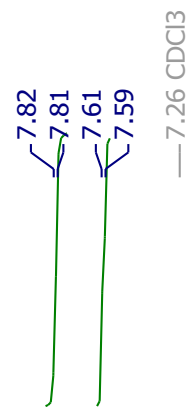

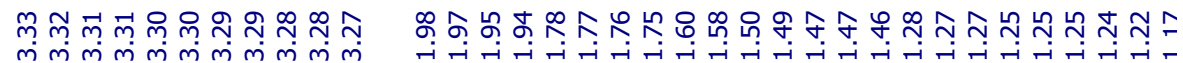

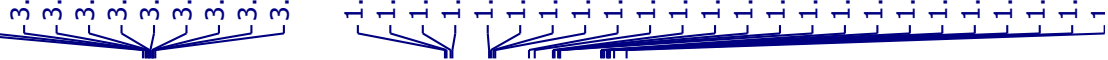

\section{$\int \quad \int 111$}
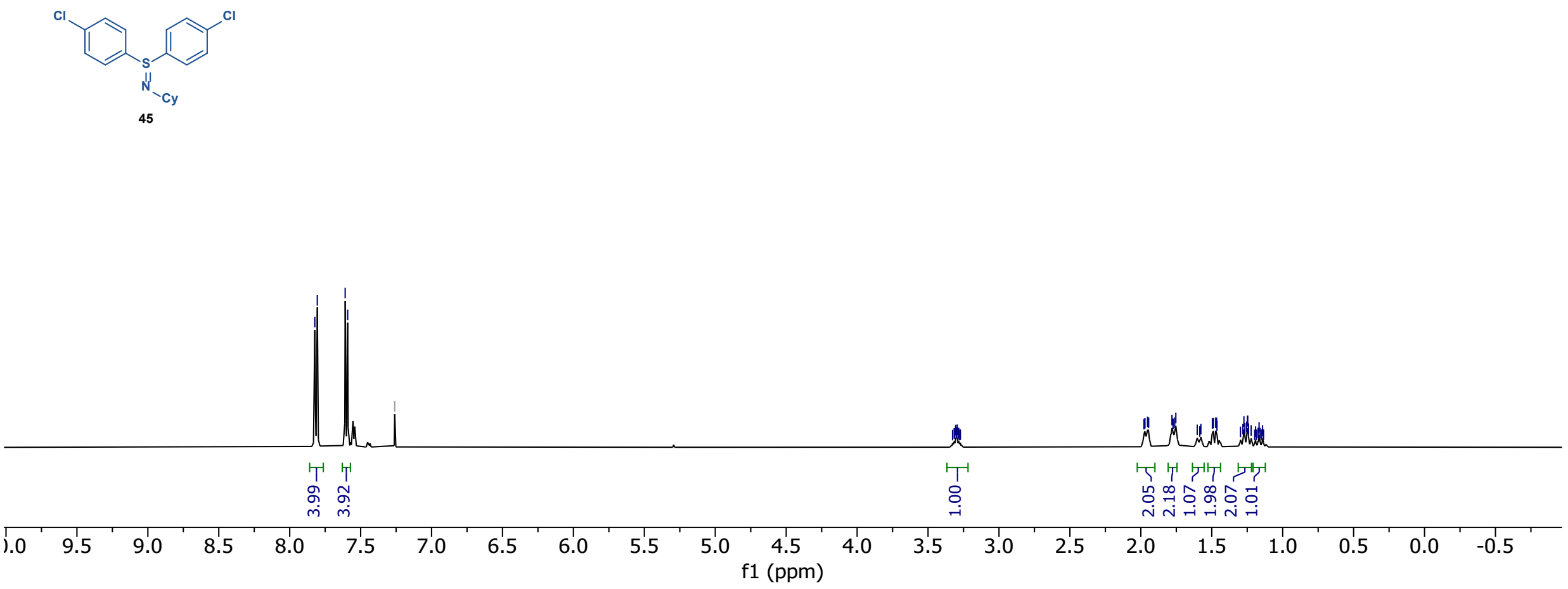
${ }^{13} \mathrm{C}$ NMR of 1,1-bis(4-chlorophenyl)-N-cyclohexylsulfanimine (45)

$\mathrm{CDCl}_{3}, 25^{\circ} \mathrm{C}$
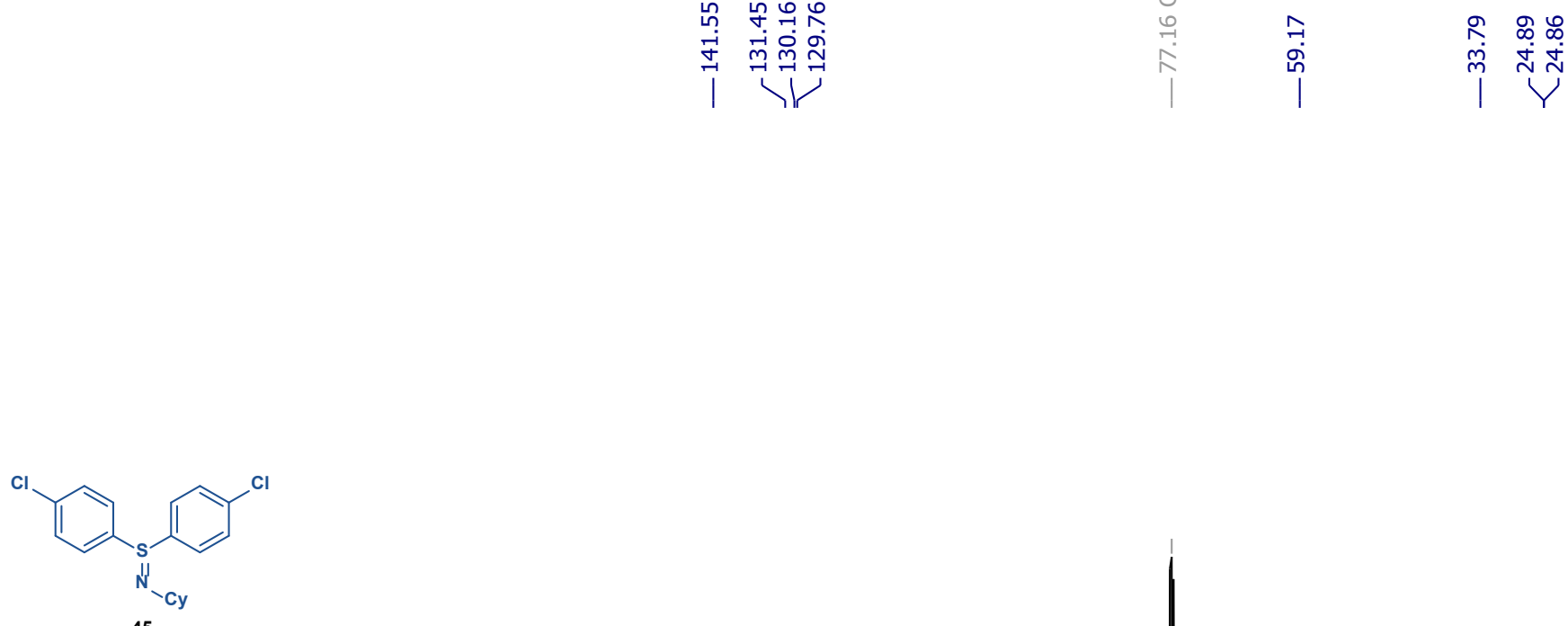

45

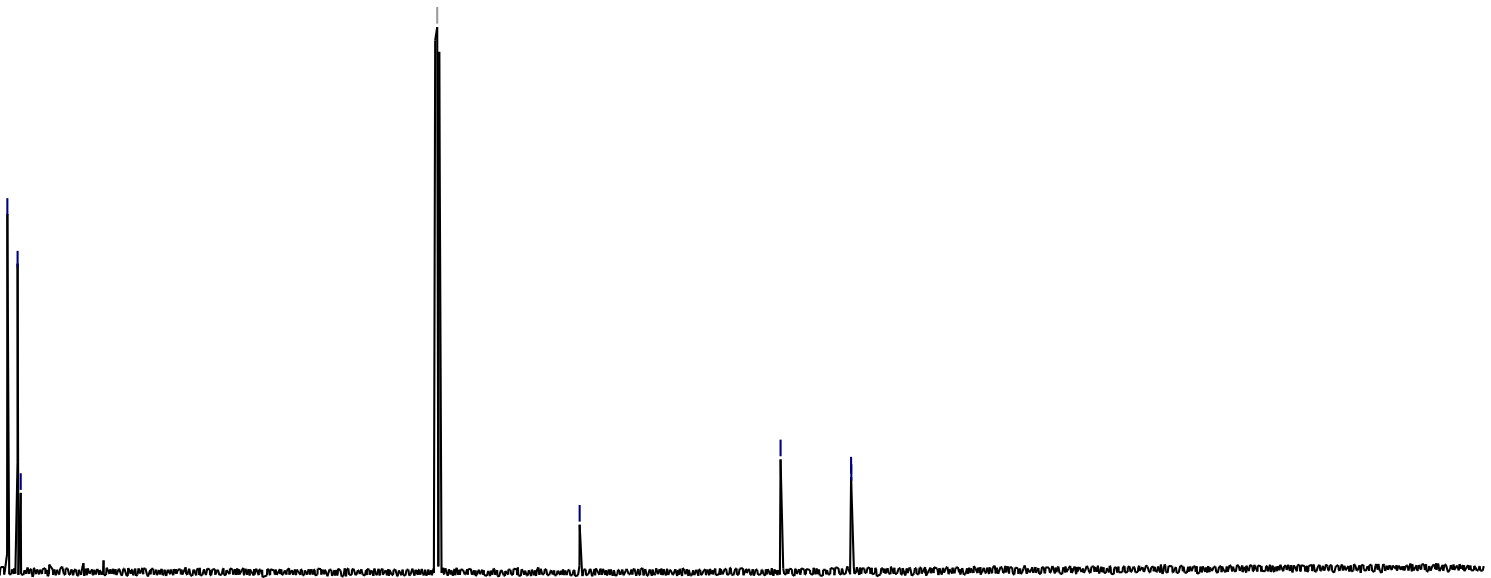

\begin{tabular}{llllllllllllllllllllllllllllllllll}
\hline & 250 & 240 & 230 & 220 & 210 & 200 & 190 & 180 & 170 & 160 & 150 & 140 & 130 & 120 & 110 & 100 & 90 & 80 & 70 & 60 & 50 & 40 & 30 & 20 & 10 & 0 & -10 & -20 & -30 & -40 & -50
\end{tabular} f1 (ppm) 


\section{REFERENCES}

1. Fulmer, G. R.; Miller, A. J. M.; Sherden, N. H.; Gottlieb, H. E.; Nudelman, A.; Stoltz, B. M.; Bercaw, J. E.; Goldberg, K. I. NMR Chemical Shifts of Trace Impurities: Common Laboratory Solvents, Organics, and Gases in Deuterated Solvents Relevant to the Organometallic Chemist. Organometallics 2010, 29, 2176-2179.

2. Berger, F.; Plutschack, M. B.; Riegger, J.; Yu, W.; Speicher, S.; Ho, M.; Frank, N.; Ritter, T. Site-Selective and Versatile Aromatic C-H Functionalization by Thianthrenation. Nature 2019, 567, 223-228.

3. Álvarez-Calero, J. M; Jorge, Z. D.; Massanet, G. M. TiCl4/Et3N-Mediated Condensation of Acetate and Formate Esters: Direct Access to $\beta$ Alkoxy- and $\beta$-Aryloxyacrylates. Org. Lett. 2016, 18, 6344-6347.

4. Julia, M.; Descoins, C. Homoallylic rearrangement with participating groups. I. Acid-catalyzed isomerization of cis and trans-2-[2'-(but-3"-enyl) cyclopropyl]-2-propanols. Bull. Soc. Chim. Fr. 1970, 5, 1805-1815.

5. Tao, C.; Lv, A.; Zhao, N.; Yang, S.; Liu, X.; Zhou, J.; Liu, W.; Zhao, J. Ligand-Free Copper-Catalyzed Synthesis of Diaryl Thioethers from Aryl Halides and Thioacetamide. Synlett 2011, 134-138.

6. Li, J.; Chen, J.; Sang, R.; Ham, W.-S.; Plutschack, M. B.; Berger, F.; Chabbra, S.; Schnegg, A.; Genicot, C.; Ritter, T. Photoredox catalysis with aryl sulfonium salts enables site-selective late-stage fluorination. Nat. Chem. 2020, 12, 56-62.

7. Neese, F. The ORCA program system. WIREs Comput. Mol. Sci. 2012, 2, 73-78.

8. Becke, A. D. Density - functional thermochemistry. III. The role of exact exchange. J. Chem. Phys. 1993, 98, 5648-5652.

9. Lee, C., Yang, W. \& Parr, R. G. Development of the Colle-Salvetti correlation-energy formula into a functional of the electron density. Phys. Rev. $B$ 1988, 37, 785-789.

10. Grimme, S., Antony, J., Ehrlich, S. \& Krieg, H. A consistent and accurate ab initio parametrization of density functional dispersion correction (DFT-D) for the 94 elements H-Pu. J. Chem. Phys. 2010, 132, 154104.

11. Grimme, S., Ehrlich, S. \& Goerigk, L. Effect of the damping function in dispersion corrected density functional theory. J. Comput. Chem. 2011, $32,1456-1465$.

12. Weigend, F. Accurate Coulomb-fitting basis sets for H to Rn. Phys. Chem. Chem. Phys. 2006, 8, 1057-1065. 
13. Weigend, F. \& Ahlrichs, R. Balanced basis sets of split valence, triple zeta valence and quadruple zeta valence quality for $\mathrm{H}$ to $\mathrm{Rn}$ : Design and assessment of accuracy, Phys. Chem. Chem. Phys. 2005, 7, 3297-3305.

14. Valeev, E. F. Libint: A library for the evaluation of molecular integrals of many-body operators over Gaussian functions, http://libint.valeyev.net/

15. Barone, V. \& Cossi, M. Quantum calculation of molecular energies and energy gradients in solution by a conductor solvent model, J. Phys. Chem. A 1998, 102, 1995-2001.

16. Hanwell, M. D. et al. Avogadro: an advanced semantic chemical editor, visualization, and analysis platform. J. Cheminf. 2012 , 4, 17. 